\title{
DISSERTATION
}

\section{Tropospheric delay parameters derived from GNSS-tracking data of a fast-moving fleet of trains}

\author{
Ausgeführt zum Zwecke der Erlangung des akademischen Grades eines Doktors der \\ technischen Wissenschaften unter der Leitung von
}

Ao.Univ.Prof. Dipl.-Ing. Dr.techn. Robert Weber

am E 120-4

Department für Geodäsie und Geoinformation

eingereicht an der Technischen Universität Wien

Fakultät für Mathematik und Geoinformation

von

Matthias Aichinger-Rosenberger

Matr.Nr: 00926550

Ried 14/7

6306 Söll

Wien, Februar 2021 
"Wonach du sehnlich ausgeschaut,

Es wurde dir beschieden.

Du triumphierst und jubelst laut Jetzt hab ich endlich Frieden! Ach, Freundchen, rede nicht so wild, Bezähme deine Zunge!

Ein jeder Wunsch, wenn er erfüllt, Kriegt augenblicklich Junge." —Wilhelm Busch (1832 - 1908) 


\section{Acknowledgements}

The verse on the previous page, composed by the famous German writer Wilhelm Busch, I had stumbled upon a while ago in some old book at home. From there on, I knew that I needed to add it to this thesis as an opening line since there's practically no better way of describing my academic career over the years. And as I'm writing this and thereby finish this chapter, there's already something new and exciting coming up for the future. Nevertheless before that is now the time to thank everybody who made this thesis and my $\mathrm{PhD}$ studies possible.

First and foremost, I would like to thank Prof. Robert Weber, who served as my supervisor and mentor not only for this thesis but at all aspects of university life. Thanks for giving me the opportunity to join the research division and pursue such an interesting $\mathrm{PhD}$ study.

Furthermore thanks to:

Johannes Böhm, again for the chance to start an academic career at TU Wien and for competent advice with his expert knowledge in atmospheric modelling and space geodesy.

Marcus Franz Glaner, for the great camaraderie and collaboration at work and numerous precious advices and discussions on GNSS, PPP and even not very "work-specific passions" like distance running.

Gregor Möller, for a lot of expert advice on all things concerning GNSS (and GNSS Meteorology in particular) as well as the great collaboration not limited by our relatively short time at the institute together.

Natalia Hanna, for a lot of expert advice on data assimilation and carrying out the assimilation tests for this thesis.

Zoreh Adavi, for providing ray-traced tropospheric delays for the validation of the results.

In general to all other colleagues at the research division and the institute who I had the pleasure to know and work with. Although my time there ended already a year ago, I really enjoyed every day of it and will always be grateful for this experience. 
Finally I would like to thank my family:

Eva, for your love and continuous support, without which this thesis would not have been possible/finished in such a short timeframe. I love you.

Paula and Fiona, the greatest gifts of my life. I love you too.

My parents, brothers and all other relatives who supported me and kept me motivated over all those years I've now spent studying. 


\section{Abstract}

Water vapour denotes one of the most important parameters utilized for describing the state and evolution of the atmosphere. Therefore, detailed knowledge of its distribution is of immense importance for weather forecasting. Furthermore, it is also the most effective greenhouse gas and highly variable in both space and time. Thus, it is obvious that high resolution observations are crucial for accurate precipitation forecasts, especially for short-term prediction of severe weather. Although not intentionally built for this purpose, Global Navigation Satellite Systems (GNSS) have proven to meet those requirements. The derivation of meteorological parameters from GNSS observations is based on the fact that electromagnetic signals are delayed when travelling through the atmosphere. Parametrisations of these delays, most prominently the Zenith Total Delay (ZTD) parameter, have been studied extensively and proven to provide substantial benefits for atmospheric research and especially Numerical Weather Prediction (NWP) model performance. Typically, regional/global networks of static reference stations are utilized to derive ZTD along with other parameters of interest in GNSS analysis (e.g. station coordinates). Results are used as a contributing data source for determining the initial conditions of NWP models, a process referred to as Data Assimilation (DA).

This thesis goes beyond the approach outlined above, showing how reasonable tropospheric parameters can be derived from highly-kinematic, single-frequency (SF) GNSS data. This data was gathered on trains of the Austrian Federal Railways (ÖBB) and processed using the Precise Point Positioning (PPP) technique. The special nature of the observations yields a number of additional challenges, from appropriate pre-processing and extended outlier detection, to advanced strategies in the PPP setup and for usage in DA procedures. Furthermore, since only SF data is provided, the treatment of the ionosphere represents one of the major challenges of this study. Therefore different strategies have been investigated and shown to provide satisfactory results, suitable for ZTD estimation.

Despite these challenging circumstances, reasonable results for ZTD estimates could be 
obtained for the analysed test cases investigating different PPP processing strategies. For validation of the results, comparisons with ZTD calculated using data from ERA5, the latest reanalysis of the European Centre for Medium-Range Weather Forecasts (ECMWF), were carried out. They yield very high correlation and an overall agreement from the low millimetre-range up to 5 centimetre, depending on solution and analysed travelling track. First tests of assimilation into a NWP model again show the reasonable quality of the results as between $30-100 \%$ of the observations are accepted by the model. Furthermore guidelines to an operational processing and possible extensions to advanced tropospheric parameters were outlined, in order to exploit the huge benefits (horizontal/temporal resolution) of this specific dataset for operational weather forecasting. 


\section{Kurzfassung}

Atmosphärischem Wasserdampf kommt durch seine Rolle als wichtigstes Treibhausgas und seine gleichzeitig räumlich und zeitlich hoch variable Verteilung eine besondere Bedeutung in der Meteorologie zu. Dadurch ist auch verständlich dass ein möglichst dichtes Beobachtungsnetz für die Niederschlagsvorhersage von entscheidender Bedeutung ist. Wenn auch ursprünglich nicht zu diesem Zweck entwickelt, können Global Navigation Satellite Systems (GNSS) hier Abhilfe schaffen. Die Bestimmung von atmosphärischen Wasserdampfmengen mit GNSS basiert dabei auf dem Sachverhalt dass elektromagnetische Signale auf ihrem Weg durch die Atmosphäre durch die Präsenz von Wasserdampf verzögert werden. Parametrisierungen dieser Verzögerung, wie der Zenith Total Delay (ZTD), können aus Netzwerken fixer Stationen in der GNSS Auswertung, zusammen mit z.B. Stationskoordinaten, bestimmt werden. Die gewonnenen Resultate werden typischerweise als komplementärer Datensatz zur Bestimmung der Anfangsbedingungen in numerischen Wettermodellen verwendet, ein Prozess der Datenassimilation bezeichnet wird.

Die vorliegende Arbeit geht über diese Ansätze hinaus indem sie die Nutzbarkeit von Einfrequenz-GNSS-Daten, welche in einem hoch kinematischen Umfeld beobachtet wurden, zur Bestimmung von ZTD zeigt. Dabei handelt es sich um Lokalisierungsdaten von Zügen der Österreichischen Bandesbahnen (ÖBB), welche mittels Precise Point Positioning (PPP) ausgewertet wurden. Die große Anzahl der bereits mit GNSS Empfängern ausgestatteten Züge verspricht dabei eine noch nie erreichte räumliche (und auch zeitliche) Auflösung von ZTD Daten über ganz Österreich. Die speziellen Eigenschaften der Daten bringen allerdings eine Reihe von Herausforderungen mit sich, von einer deutlich aufwendigeren Ausreißerkorrektur bis hin zu speziellen Prozessierungs- und Assimilierungsansätzen welche in dieser Arbeit getestet wurden. Durch die Verwendung von Einfrequenz-Daten kommt der Korrektur von ionosphärischen Laufzeitverzögerungen eine besonders hohe Bedeutung zu. Aus diesem Grund wurden hierfür mehrere verschiedene Ansätze getestet, welche alle zufriedenstellende Ergebnisse 
viii

liefern konnten.

Trotz dieser herausfordernden Voraussetzungen konnten durchwegs realistische Ergebnisse für die analysierten Testfälle erzielt werden. Vergleiche mit aus atmosphärsichen Reanalyse-Daten (ERA5) des European Centre for Medium-Range Weather Forecasts (ECMWF) gerechneten ZTDs zeigen eine sehr hohe Korrelation und mittlere Abweichungen vom unteren mm-Bereich bis zu $5 \mathrm{~cm}$, abhängig von verwendeter Software und Teststrecke. Erste Tests von Assimilation in ein numerisches Wettermodell zeigen dass, abhängig von Lösung und Strecke, 30 - 100\% der Beobachtungen vom Modell akzeptiert werden. Darüber hinaus wurde auch noch eine Strategie für die Implementierung dieser Idee im operationellen Betrieb entwickelt bzw. vorgeschlagen, um die großen Vorteile dieser Datensätze (sehr hohe räumliche/zeitliche Auflösung) für die operationelle Wettervorhersage nutzbar zu machen. 


\section{Contents}

Acknowledgements iii

Abstract $\quad$ v

Kurzfassung vii

Contents viii

1 Introduction $\quad 1$

1.1 Thesis contribution . . . . . . . . . . . . . . . . . . 3

1.1 .1 Goals ........................ 4

1.2 Thesis outline . . . . . . . . . . . . . . . . 5

2 Global Navigation Satellite Systems $\quad 7$

2.1 Principles of GNSS positioning . . . . . . . . . . . . . . . . . . . . . . 8

2.2 GNSS types . . . . . . . . . . . . . . . . . . . . . . . . . . . . . . . 8

2.2 .1 GPS . . . . . . . . . . . . . . . . 9

2.2 .2 GLONASS . . . . . . . . . . . . . . . . 12

2.2 .3 Galileo . . . . . . . . . . . . . . . . . . . 13

2.3 Precise Point Positioning . . . . . . . . . . . . . . . . . . . . . . . . . 14

2.3.1 Observation equations . . . . . . . . . . . . . . . . . 15

2.3.2 Error sources . . . . . . . . . . . . . . . . . . . . . . . . . . 15

2.3.3 Linear Combinations of GNSS Observations . . . . . . . . . . . 19

2.3.4 Integer ambiguity resolution . . . . . . . . . . . . . . . . 22

2.3 .5 Kinematic PPP . . . . . . . . . . . . . . . . . . . . . . 22

3 Physics of the atmosphere 23

3.1 General overview . . . . . . . . . . . . . . . . . . . . . . 23

3.1.1 Optical properties and energy budget . . . . . . . . . . . . 23

3.1 .2 Atmospheric composition . . . . . . . . . . . . . . . . . 24 
3.2 Vertical structure and layers of the atmosphere . . . . . . . . . . 25

3.2.1 Hydrostatic balance . . . . . . . . . . . . . . . . . . . 25

3.2.2 Vertical structure of pressure/density . . . . . . . . . . . 2 26

3.2.3 Vertical distribution of temperature . . . . . . . . . . . . . . 27

3.2 .4 Atmospheric layers . . . . . . . . . . . . . . . . . . . . . . . . 27

3.3 Water vapour in the atmosphere . . . . . . . . . . . . . . . . . 29

3.3.1 Water vapour measures . . . . . . . . . . . . . . . . . . . 30

3.4 Precipitation . . . . . . . . . . . . . . . . . . . . . . . . . . 32

3.4.1 Numerical modelling of precipitation . . . . . . . . . . . 33

4 Signal propagation in the atmosphere 35

4.1 Maxwell equations . . . . . . . . . . . . . . . . . . . . . . . 35

4.2 Path delays . . . . . . . . . . . . . . . . . . . . . . . . 36

4.2 .1 lonospheric delay . . . . . . . . . . . . . . . . . . . . 37

4.2.2 Tropospheric delay . . . . . . . . . . . . . . . . . . . . . . 39

5 GNSS Meteorology 45

5.1 History . . . . . . . . . . . . . . . . . . . . . . . . . 45

5.2 GNSS-based troposphere products for NWP . . . . . . . . . . . . 46

5.2.1 Ground-based products . . . . . . . . . . . . . . . . . . . 47

5.2.2 Space-based products . . . . . . . . . . . . . . . . . . . 49

5.3 Estimation of tropospheric parameters using PPP . . . . . . . . . . . . 50

5.3 .1 Kinematic platforms . . . . . . . . . . . . . . . 52

6 GNSS data processing 53

6.1 Data sources . . . . . . . . . . . . . . . . . . . . . . 53

6.1 .1 Greenlight . . . . . . . . . . . . . . . . 53

6.1 .2 Railway track database . . . . . . . . . . . . . . . . . 55

6.1 .3 EPOSA .............................. 55

6.2 Pre-Processing . . . . . . . . . . . . . . . . . 56

6.2 .1 Dilution of Precision . . . . . . . . . . . . . . . . . . 56

6.2 .2 Multipath analysis . . . . . . . . . . . . . . . . 57

6.2 .3 Cycle slip detection . . . . . . . . . . . . . . . . . . 58

6.2 .4 Data cleaning . . . . . . . . . . . . . . . . . . . . . . . . . 59

6.3 Parameter estimation in PPP processing . . . . . . . . . . . . . . . . 59

6.3 .1 Least-squares adjustment . . . . . . . . . . . . . . . . . . 60

6.3 .2 Kalman Filtering . . . . . . . . . . . . . . . . . . . . . 62 
6.4 SEID . . . . . . . . . . . . . . . . . . . . . . . . 63

6.4.1 Accuracy of SEID model . . . . . . . . . . . . . . . . . 65

6.4 .2 Kinematic implementation . . . . . . . . . . . . . . . . 66

6.5 Processing scheme: CSRS . . . . . . . . . . . . . . . . . . 66

6.6 Processing scheme: PPP-Wizard . . . . . . . . . . . . . . . . . . 67

6.7 Processing scheme: GAMP using SEID . . . . . . . . . . . . . . 68

6.7 .1 GAMP . . . . . . . . . . . . . . . . . . 69

6.8 Processing scheme: Height-constrained PPP . . . . . . . . . . . . . . . 69

6.9 Filtering methods and performance metrics . . . . . . . . . . . . . . 71

6.9.1 Moving average filtering . . . . . . . . . . . . . . . . . 71

6.9.2 Error metrics for performance evaluation . . . . . . . . . . . . 72

7 ZTD validation and assimilation $\quad 75$

7.1 Validation using ERA5 reanalysis products . . . . . . . . . . 76

7.1 .1 ERA5 . . . . . . . . . . . . . . . . 76

7.1.2 ZTD calculation from ERA fields . . . . . . . . . . . . . 77

7.1 .3 Quality of ERA5-derived ZTD . . . . . . . . . . . . . 78

7.2 Basics of data assimilation . . . . . . . . . . . . . . . . . . . 80

7.2 .1 DA techniques . . . . . . . . . . . . . . . . 81

7.3 Assimilation of GNSS data in NWP . . . . . . . . . . . . . . . . . 83

7.4 Assimilation and verification methodology . . . . . . . . . . . . . . . . . 84

7.4.1 Model description and setup . . . . . . . . . . . . . . . . . 84

8 Results $\quad 87$

8.1 Overview of conducted case studies . . . . . . . . . . . . . . . . . . 87

8.1 .1 Test cases . . . . . . . . . . . . . . . . . . . . 88

8.1 .2 PPP processing settings . . . . . . . . . . . . . . . . . 89

8.2 Case study 1: $11.05 .2017 \ldots \ldots$. . . . . . . . . . . . . . . . . . . 92

8.2.1 Raw data quality . . . . . . . . . . . . . . . . . . . . . . 92

8.2.2 PPP noise analysis . . . . . . . . . . . . . . . . . . . . 93

8.2 .3 ZTD validation . . . . . . . . . . . . . . . . . . . . . 95

8.3 Case study 2: $28.09 .2017 \ldots \ldots$. . . . . . . . . . . . . . . . 100

8.3.1 Raw data quality . . . . . . . . . . . . . . . . . . . . 100

8.3.2 PPP noise analysis . . . . . . . . . . . . . . . . . . . . . 101

8.3.3 SEID processing . . . . . . . . . . . . . . . . . 104

8.3 .4 ZTD validation . . . . . . . . . . . . . . . . . 106

8.4 Case study 3: $29.11 .2019 \ldots \ldots 110$ 
8.4.1 Raw data quality . . . . . . . . . . . . . . . . . . . . . . 110

8.4.2 PPP noise analysis . . . . . . . . . . . . . . . . . . . . 112

8.4 .3 ZTD validation . . . . . . . . . . . . . . . . . . . . . 113

8.5 Case study 4: $01.12 .2019 \ldots 116$

8.5.1 Raw data quality . . . . . . . . . . . . . . . . 116

8.5 .2 PPP noise analysis . . . . . . . . . . . . . . . . . . . . . 118

8.5 .3 ZTD validation . . . . . . . . . . . . . . . . . . 122

8.6 ZTD sensitivity analysis . . . . . . . . . . . . . . . . . . . . . . . . 124

8.6.1 Impact of GLONASS observations . . . . . . . . . . . . . . . 124

8.6.2 Elevation cut-off . . . . . . . . . . . . . . . . . . 127

8.6.3 Estimation of horizontal gradients . . . . . . . . . . . . . . . 129

8.6.4 Mis-modelling of ionosphere using SEID . . . . . . . . . . . . 131

8.6.5 Altitude-dependency of estimation errors . . . . . . . . . . . . 132

8.7 Assimilation tests in WRF . . . . . . . . . . . . . . . . . 135

8.7 .1 WRF setup . . . . . . . . . . . . . . . . 135

8.7.2 Influence of horizontal resolution . . . . . . . . . . . . 135

8.7 .3 Assimilation test: CS3 . . . . . . . . . . . . . . . . 138

8.7 .4 Assimilation test: CS2 . . . . . . . . . . . . . . . . . . . 138

9 Towards real-time processing $\quad 143$

9.1 Required adaptations for NRT GNSS processing . . . . . . . . . . . . 144

9.1.1 Data transmission . . . . . . . . . . . . . . . . . . . . . 144

9.1 .2 Orbit/clock products . . . . . . . . . . . . . . . . . . 144

9.1 .3 Processing software . . . . . . . . . . . . . . . . . . . . . 144

9.1.4 Output interval . . . . . . . . . . . . . . . . . . . . 145

9.1.5 Data format of NRT products . . . . . . . . . . . . . . 146

9.2 Purposed workflow for NRT processing . . . . . . . . . . . . . . 146

9.2.1 SF-PPP using regional ionosphere maps . . . . . . . . . . . . . 146

9.2 .2 DF-PPP using SEID model . . . . . . . . . . . . . . . . . 146

9.3 Requirements for NWP assimilation and applications . . . . . . . . . . 148

9.3.1 DA configuration . . . . . . . . . . . . . . . . . . . . . . . . . 148

9.4 Extension to advanced tropospheric parameters . . . . . . . . . . . . . 150

10 Conclusions and outlook 151

$\begin{array}{ll}\text { Acronyms } & 157\end{array}$

$\begin{array}{ll}\text { Bibliography } & 171\end{array}$ 
Curriculum Vitae 


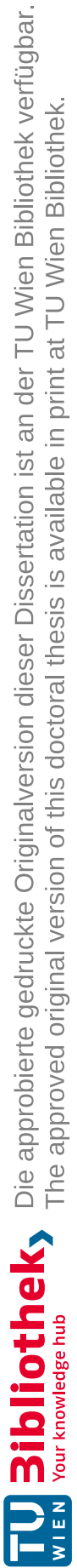




\section{Chapter 1}

\section{Introduction}

In times of extreme weather events and climate change, monitoring of the Earth's atmosphere is becoming a more crucial task than ever before. One of the most important, yet also at least understood ingredients of this complex system is water vapour. It is not only the key parameter in formation of clouds and thus precipitation, but also the most important greenhouse gas affecting global warming. In relation to its importance, observations of atmospheric water vapour are relatively sparse. Radiosondes and other measurement techniques are typically used to observe its concentration and distribution, but these techniques often suffer from low temporal and horizontal resolution, e.g. providing measurements only once a day.

This is where the field of Global Navigation Satellite Systems (GNSS) can provide an alternative. The retrieval of water vapour from GNSS signals relies on the fact that electromagnetic waves are delayed on their way through the atmosphere. In terms of the neutral atmosphere, this delay originates due to the presence of water and thus can be interpreted as a measure of water vapour in the column above the receiver.

Tropospheric parameters, derived from GNSS sensors, are a well-established data source for Numerical Weather Prediction (NWP) nowadays. The widespread usage of GNSS systems for multiple fields of application provides a huge amount of observations available for the meteorological community. Based on the concept of GNSS Meteorology, outlined by Bevis et al. (1992), tropospheric delays are estimated operationally both in national and international networks, like the European EGVAP network (Guerova et al. 2016). Assimilation of these observations in NWP models is nowadays a widely-used technique to improve precipitation and cloud cover forecasts. Several new developments have been achieved in the last two decades, e.g. the establishment of GNSS station networks used solely for atmospheric monitoring or the usability of the Galileo system. Tropospheric estimates are typically derived within a double-difference (DD) network 
solution, which can provide the high accuracy needed in NWP $(\sim 1 \mathrm{~cm}$ for ZTD). This approach provides the most accurate results but has the downside of being cost-intensive since only high-end infrastructure can be used and a larger number of reference stations is needed for a stable solution.

Aside of this 'classical' approach, several authors have also studied the potential of low-cost GNSS receivers in terms of tropospheric monitoring. Therefore, Precise Point Positioning (PPP) denotes the most promising approach for processing nowadays, especially when results have to be available in (near) real-time. The processing does not need any data from reference stations and thus computational speed is increased remarkably. Another advantage is the fact that tropospheric estimates from PPP are not biased by correlations as typically introduced in small-scale local station networks when applying DD processing (Möller 2017). These can prevent the detection of local (site-specific) water vapour trends/anomalies and therefore can be problematic for investigation very small-scale weather phenomena. However, when using PPP, high quality information of satellite orbits and clocks (provided by institutions like the International GNSS Service (IGS)) is mandatory to obtain results with high accuracy.

Low-cost receivers often only provide single-frequency (SF) observations. A major problem when working with SF data is the treatment of the non-neutral atmosphere, the so-called ionosphere. For dual-frequency (DF) observations its effect can be eliminated by building the appropriate linear combination using observations on two frequencies. However, this is not possible with SF observations and thus the effect must be modelled. If ionospheric delays and other major error sources can be modelled with sufficient accuracy, most studies show promising results in terms of positioning as well as tropospheric monitoring.

Until today, most studies concentrated on measurements from static receivers due more trivial data processing and more controllable measurement conditions.

Gao et al. (2006) developed a real-time SF-PPP system and tested it in both road and marine environments. Both test scenarios achieved decimetre accuracy in kinematic processing mode.

de Bakker and Tiberius (2017) demonstrated lane identification and mapping on a motorway using SF-PPP. Performance was found to be strongly dependent on the number of available satellites.

Deng et al. (2009) densified an already existing station network with SF receivers and retrieved tropospheric delays using static PPP in post-processing mode. In order to deal with the ionospheric influence, they developed the Satellite-specific Epoch-differenced Ionospheric Delay (SEID) model, which uses information from surrounding reference sta- 
tions to generate synthetic observations on a second frequency for the SF receiver. This approach provided promising results and will be tested in the course of this study as well. Krietemeyer et al. (2018) studied the feasibility of using low-cost receivers to increase the density of GNSS networks for retrieval of Precipitable Water Vapour (PWV). They processed one year of GNSS data from an IGS station and two co-located single-frequency stations also by using the SEID algorithm to correct for the ionospheric influence.

Mendez Astudillo et al. (2018) carried out a performance assessment of three PPP online services and three software packages concerning ZTD estimation. In this context, they compared ZTD estimates from PPP processing to those obtained from the IGS tropospheric products. The results indicate that the online PPP services performance is better than those of selected PPP software packages in all cases.

Zhao et al. (2019) estimated both PWV from low-cost multi-GNSS receivers in static mode. They retrieved ZTDs with an accuracy better than $10 \mathrm{~mm}$ compared with troposphere products published by IGS and about $3 \mathrm{~mm}$ for PWV compared to radiosonde results.

Only very few studies have investigated the possibility of monitoring tropospheric parameters from kinematic platforms. Most of these kinematic studies used maritime environments (shipborne (Rocken et al. (2005), Boniface et al. (2012), Fujita et al. (2008), Kealy et al. (2012)) and buoys (Chadwell and Bock (2001), Dodson et al. (2001)) or airborne data (Skone et al. (2006)). Up till now, the only investigation on train-borne GNSS data was carried out by Webb et al. (2016), who analysed Zenith Wet Delay (ZWD) for over a decade of continuous observations over a range of altitudes on the Snowdon Mountain Railway, located in Snowdonia National Park, North Wales. They found an improvement of a multi-system GNSS solution in kinematic PPP, with an agreement of $11.6 \mathrm{~mm}$ for the multi-GNSS solution compared to $16.2 \mathrm{~mm}$ for the GPS-only solution in ZWD.

\subsection{Thesis contribution}

This study goes one step beyond the studies presented before by making use of the kinematic PPP approach to derive tropospheric parameters from SF observations taken on fast-moving trains. These trains are equipped with dual-system SF receivers, which currently are able to track GPS and GLONASS signals. In a future state the receivers will be switched to GPS/GALILEO capability. Data is gathered on different railway tracks over Austria in the course of the project Greenlight, led by the Austrian Federal Railways (ÖBB). 
A higher number of challenges is faced due to the fast movement of the sensor and a large number of obstructed areas on the track (e.g. tunnels). The potential benefits are a very high spatial and temporal resolution for observations gathered at train tracks covering huge parts of Austria and neighbouring countries. Moreover, a high number of trains $(\sim 1400)$ is already equipped with GNSS sensors and up to 200 vehicles more are planned in the course of the next year.

In particular this thesis aims to answer the following questions:

- Can reliable tropospheric estimates be derived from GNSS tracking data of fastmoving trains?

- Which pre-processing steps are required to prepare the obtained tracking data for PPP processing (cycle slip detection, multipath, ionospheric corrections)?

- Which data processing scheme(s) is/are adequate to obtain results comparable to independent ZTD sources (NWP, ray-tracing)?

- What is the quality of the derived data and is it sufficient to be incorporated into an NWP data assimilation system?

- Can the estimation and assimilation be carried out in a (near) real-time (NRT) operational mode?

First investigations, as presented in Aichinger-Rosenberger and Weber (2020), already indicated the possibility to derive ZTD estimates of reasonable quality, even for railway tracks in highly challenging environments. This thesis extends these investigations for a number of test tracks and processing schemes, analyses specific sensitivities in the GNSS processing and presents first tests of ZTD assimilation into a NWP model.

\subsubsection{Goals}

Directly linked to those questions raised above are the following goals of the thesis:

\section{Goals related to GNSS processing:}

- Data cleaning: Implement a strategy for data cleaning, mostly concerning cycle slips and multipath

- GNSS data processing: Find the best possible processing scheme(s) for the given GNSS observations, especially taking into account adequate modelling of ionospheric corrections 
- Tropospheric estimation: Estimate tropospheric parameters (ZTD) using the developed processing scheme(s)

- Quality assessment: Assess the limits of reachable quality for tropospheric parameter estimates using these processing scheme(s) by comparison to NWP-derived and ray-traced tropospheric delays

- Processing of test cases (train tracks): Process data for a number of case studies (available train tracks) in post-processing mode with focus on tropospheric estimation

- NRT GNSS data processing: Provide strategy and theoretical basis of possible extension from post-processing to NRT operation

\section{Goals related to NWP data assimilation:}

- Observation quality check: Assess if quality of estimates is sufficient to enter the model

- Assimilation case studies: Assimilation of GNSS tropospheric estimates from moving trains into a NWP model

\subsection{Thesis outline}

Chapter 2 gives an introduction on the most important aspects of existing GNSS, the physical/geometrical principles behind them and their usage in different scientific and real-world applications.

Chapter 3 summarizes information on the Earth's atmosphere and it's governing processes which are of importance for this study.

Chapter 4 introduces the theoretical background and physical laws behind the propagation of electromagnetic signals travelling through the atmosphere.

Chapter 5 takes a closer look on the history and the latest scientific achievements in the specific field of GNSS Meteorology.

Chapter 6 describes the different processing schemes for GNSS data used in this study. A detailed description of processing setups is given for all deployed approaches. Furthermore the utilized data and necessary pre-processing steps are introduced.

Chapter 7 introduces the methods and datasets used for assessing the reached quality of the tropospheric estimates derived in Chapter 6 . Results of this validation procedure can serve as an indication for the usability of the results for assimilation. Furthermore it deals with assimilation procedure of the ZTD estimates, derived from the processing 
schemes introduced in Chapter 6, into a NWP model. Therefore a short introduction to the mathematical background of data assimilation and the utilized NWP model is given. Chapter 8 provides the results from all test cases conducted. Results of data processing (in terms of ZTD and height estimates mainly) are shown as well as validation and assimilation results.

Chapter 9 aims to serve as an outlook to a possible NRT operational mode for GNSS tracking data processing. Therefore the theoretical framework and ideas for implementation as well as challenges of this approach will be examined.

Chapter 10 concludes the thesis with a discussion and summary of the main findings. Furthermore an outlook on future implementations of this research idea is provided, which incorporates recommendations from Chapter 9. 


\title{
Chapter 2
}

\section{Global Navigation Satellite Systems}

\begin{abstract}
Global Navigation Satellite Systems (GNSS) have revolutionised a number of scientific research fields as well as practical applications over the last decades (Aichinger-Rosenberger 2018). Originally starting as a completely military system, it was opened for public usage in 1983. Since then, it has been extensively used and studied in the scientific and the public society and a widespread field of applications has emerged over the last years. The following points denote just a small selection of topics which rely on or are closely related to GNSS:
\end{abstract}

- Navigation: reaching from pedestrian to automotive/railway/aviation usage

- High-precision positioning

- High-precision timing applications

- Assisted/Autonomous driving

- Geomonitoring: detection of plate tectonics, earthquakes, landslides

- Atmospheric monitoring: weather forecasting, climate research

The following section introduces the principle of GNSS positioning, which provides the foundation for all applications mentioned above. Based on this principle the observation equations are formulated and the typical error sources are described. Furthermore the different types of GNSS are outlined.

At last, the main processing scheme used in this study is outlined, Precise Point Positioning (PPP). There, a detailed description of the observables, governing equations and estimated parameters of the approach is given. Benefits and weaknesses of the technique as well as typical error sources influencing its performance are outlined. 


\subsection{Principles of GNSS positioning}

The basic principle of GNSS positioning relies on very precise propagation time measurements for an electromagnetic signal travelling from a satellite to a receiver. Through exact knowledge of this propagation time $\Delta t$ the geometric distance $r$ from satellite to receiver results from

$$
r=c\left(T_{u}-T_{s}\right)=c \Delta t
$$

where $c$ denotes the speed of light, $T_{u}$ the system time at which the signal left the satellite and $T_{s}$ the system time when it reaches the receiver. However, since the receiver and satellite clocks are not synchronized the measurements of propagation time will be biased. Thus the calculated distance will not be the real geometric distance $r$ but the so-called pseudorange $\rho$. It is given by

$$
\rho=r+c\left(\delta t_{u}-\delta t^{s}\right)
$$

where $\delta t_{u}$ is the offset of the receiver clock from system time and $\delta t^{s}$ is the offset of the satellite clock from system time. Since on board of the satellites atomic clocks are used, the error in the satellite clock is marginal compared to the receiver clock. Moreover it typically cancels through observation differencing or can be modelled using accurate correction models (see Section 2.3 for more details). Therefore only $\delta t_{u}$ is left in Equation 2.2,

$$
\rho=r+c \delta t_{u}
$$

which now consists of four unknowns ( $X, Y$ and $Z$ coordinate of receiver position and receiver clock error). Thus, pseudorange observations from at least four satellites $\left(\rho_{1}, . ., \rho_{4}\right)$ enable the calculation of the receiver coordinates. Additional observations result in an over-determined problem which is typically solved by least-squares adjustment.

Geometrically this concept corresponds to the intersection of the surfaces of spheres, which have the measured pseudoranges as radius. This is indicated in Figure 2.1, simplified to the 2D case (using circles instead of spheres). Typically only three satellites would be sufficient to calculate the 3D receiver position, as three spheres would intersect in one point on Earth. Nevertheless, introducing the receiver clock error as an unknown like mentioned before gives a minimum number of four satellites needed.

\subsection{GNSS types}

Since the start of their public availability in 1983, multiple GNSS types have been built up. These include the American GPS, Russian system GLONASS, European system 


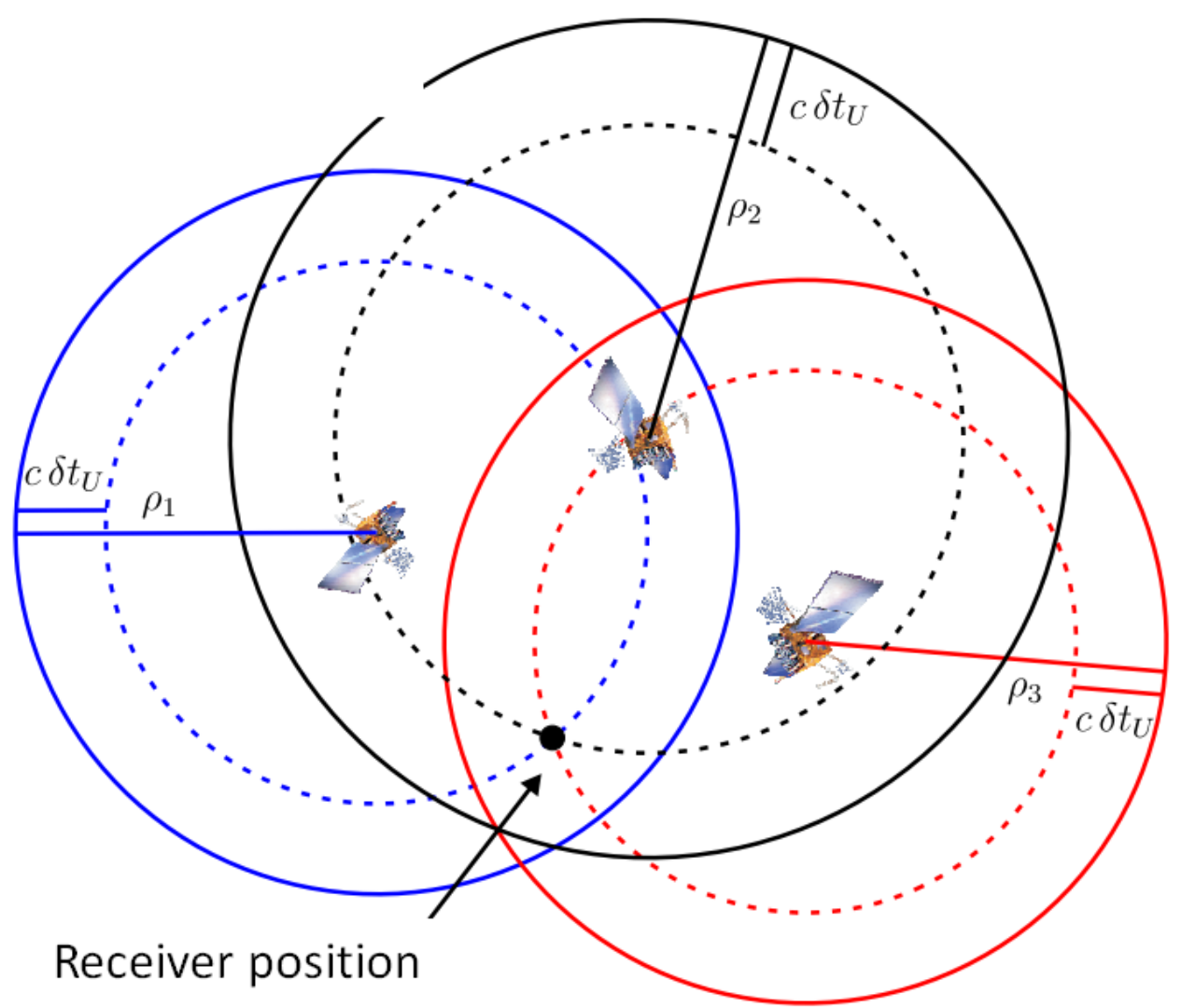

Figure 2.1: Geometric concept of GNSS positioning, shown here in the 2D case. Taken from Böhringer (2008)

Galileo and the Chinese system Beidou. The first three mentioned systems are described in more detail in the next section.

\subsubsection{GPS}

Since the early 1960s, several U.S government organizations, including the Department of Defence (DOD), were interested in developing a satellite system for three-dimensional position determination (Kaplan and Hegarty 2006) as the successor of the already operating Transit Satellite Navigation System. The aim was to secure the following attributes for the system:

- global coverage

- continuous/all-weather operation

- ability to serve high-dynamic platforms

- high accuracy 


\begin{tabular}{c|c} 
Component & Frequency \\
\hline Fundamental frequency & $f_{0}=10.23 \mathrm{MHz}$ \\
Carrier L1 & $154 \cdot f_{0}=1575.42 \mathrm{MHz}$ \\
Carrier L2 & $120 \cdot f_{0}=1227.60 \mathrm{MHz}$ \\
Carrier L5 & $115 \cdot f_{0}=1176.45 \mathrm{MHz}$
\end{tabular}

Table 2.1: Components and frequencies for civil use of GPS signal

In 1969, the Defense Navigation Satellite System (DNSS) program was established, an effort to concentrate the contributions of different organisations. As a result, the Navstar Global Positioning System (GPS) (DoD 2020), a space-borne positioning system for military purposes, was finally introduced in 1973. From then on it took another 10 years until the system became open to civil users in 1983. Since then GPS has been running as a dual-use system, for both military and civil purposes.

\section{GPS signals}

GPS satellites use the so-called code division multiple access (CDMA) technique to broadcast ranging codes and navigation messages. Every satellite emits an individual Pseudo Random Noise (PRN) code using this technique. These codes were initially solely modulated on two frequencies L1 (1575.42 MHz) and L2 (1227.6 MHz), which are multipliers of the fundamental frequency $10.23 \mathrm{MHz}$. Onto L1, Course/Acquisition $(\mathrm{C} / \mathrm{A})$ code and Precise (P1) code is modulated as well as Precise (P2) code onto L2. In the course of the GPS modernization program a new third civil frequency L5 was introduced. This opened multiple new possibilities of carrier phase combinations, especially useful for the resolution of ambiguities and elimination of the ionospheric delay. Table 2.1 shows an overview of carrier frequencies used for (civil) GPS.

In contrast to $L 5$, the $L 3=1381.05 \mathrm{MHz}$ and $\mathrm{L} 4=1379.913$ carrier frequencies are only used for military purposes (Hofmann-Wellenhof et al. 2012). Since first tests of GPS revealed much better accuracy than originally expected, techniques like Selective Availability (SA) were used to ensure superiority of the military over the civil user. This technique incorporated e.g. manipulation of orbital and satellite clock information for civil users to worsen positioning results until its use was finally terminated in May 2000. However, encrytion of various signals for military purposes (Anti-Spoofing) is still in use.

\section{System components}

In literature, GPS is usually described as a systems comprised of three different segments: 
- Space segment

- Control segment

- User segment

The space segment represents the satellite constellation, which consists nominally of 24 operational satellites placed in six nearly circular orbits inclined by $55^{\circ}$ with an altitude of about 20200 km above the Earth's surface (Karabatic 2011). This number of 24 satellites was reached in July 1995 and thereby the space segment became fully operational. Different types of satellites have been developed in the course of the last 20-30 years, which are split in different blocks. By March 2019, 31 operational satellites are available and delivering data down to Earth.

The control segment is build upon a master control station, located in Colorado Springs, USA. This master station is responsible for controlling the satellites and keeping the system operating. This includes maintaining the satellites in their proper orbital positions (called stationkeeping) and monitoring satellite subsystem health and status (Kaplan and Hegarty 2006). In addition, five monitoring stations and a number of ground antennas are employed. The monitoring stations make continuous measurements to all visible satellites and send this data to the master station. There, orbit and clock parameters are calculated and forwarded to the ground antennas, which upload the parameters three times a day. Using this data, the navigation message for GPS is generated.

The user segment incorporates every user of the system and is split into two different categories, civil and military users. As already mentioned in the beginning of this chapter, the number of applications for civil use have increased heavily over the last years and continue to do so. They reach from classical tasks like positioning, navigation or monitoring to meteorological and climatological applications. In principle, one distinguishes between single- and multi-frequency receivers depending on how many frequency bands observations can be taken.

\section{GPS reference system}

GPS coordinates are defined in the the DOD's World Geodetic System 1984 (WGS-84) (National Imagery and Mapping Agency 2000), which represents the standard physical model of the Earth used for GPS applications (Kaplan and Hegarty 2006). A list of the defining parameters of the underlying reference ellipsoid is given is Table 2.2. 


\begin{tabular}{c|c} 
Parameter and Value & Description \\
\hline$a=6378137.0 \mathrm{~m}$ & Semi-major axis of the ellipsoid \\
$f=1 / 298.257223563$ & Flattening of the ellipsoid \\
$\omega_{E}=7.292115 \cdot 10^{-5} \mathrm{rad} \mathrm{s}^{-1}$ & Angular velocity of the Earth \\
$\mu=3986004.418 \cdot 10^{8} \mathrm{~m}^{3} \mathrm{~s}^{-2}$ & Earth's gravitational constant
\end{tabular}

Table 2.2: Defining parameters of WGS-84, taken from Hofmann-Wellenhof et al. (2012)

\subsubsection{GLONASS}

Globalnaya navigatsionnaya sputnikovaya sistema (GLONASS) is the Russian counterpart to the American GPS system. Like GPS originally designed as a fully military service, its development started already in 1976. In 1982 the first satellites were launched and in 1995 the full constellation was completed. The latter was also the year in which the system became publicly available. At the end of the nineties, the capacity of the system declined more and more due to lack of financial supply. This continued until 2001, when the restoration of the system became one the top priorities of the Russian government. GLONASS is managed by the Russian Space Forces and operated by the Coordination Scientific Information Center of the Ministry of Defense of the Russian Federation (Xu 2007).

\section{GLONASS signals}

Just like GPS, GLONASS satellites transmit two types of signals, a standard-precision ( $C / A$ code) and a high-precision signal ( $P$ code). In contrast to the CDMA technique used by other GNSS systems, GLONASS uses frequency division multiple access (FDMA), which means that each satellite can be identified by its own unique carrier frequencies. These carrier frequencies (in $\mathrm{MHz}$ ) are defined by

$$
\begin{aligned}
& G 1=1602.0+0.5625 k \\
& G 2=1246.0+0.4375 k
\end{aligned}
$$

where $k$ denotes the frequency channel. In the course of modernization, the CDMA technique is being investigated since 2008. This is expected to provide multiple benefits for interoperability with other systems. In addition, a new signal on the third carrier as well as a second civil signal on G2 should be established. Since November 2018 24 GLONASS satellites are operational and thus full operational capability (FOC) is reached. 


\section{GLONASS reference system}

The terrestrial reference frame used by GLONASS is the "PZ-90" (Earth Parameters 1990 - Parametry Zemli 1990), which uses the mean position from 1990 to 1995 for the precise location of the North Pole. In contrast to this, WGS-84 as GPS reference system uses the location of the North Pole in 1984. The new version PZ-90.11 is broadcasted since 31 December 2013. It is aligned to the International Terrestrial Reference System at epoch 2011.0 at the centimeter level.

\subsubsection{Galileo}

Galileo is the European alternative to GPS and GLONASS, established by the European Union (EU) through a cooperation of the European Commission (EC), the European Space Agency (ESA) and at a later stage the European GNSS Agency (GSA). Up to date (December 2020), the system consists of 24 operational satellites. In full operational mode observations from 30 satellites should become available to users.

Contrary to its counterparts GPS and GLONASS, Galileo is intended primarily for civilian use, as basic (lower-precision) services will be free and open to everyone. From 2022 on, also high precision positioning will become available for users with multiple signal receivers, the Galileo High-Accuracy Service (HAS).

\section{Galileo signals}

The Galileo system transmits three different signals:

- E1

- E5 consisting of E5a and E5b

- E6

Similar to GPS, these carriers are based on the fundamental frequency $f_{0}=10.23 \mathrm{MHz}$. In order to enable services like open, Search-And-Rescue (SAR), commercial or public regulated services, each satellite will broadcast different navigation messages (Karabatic 2011). An overview of all available carrier frequencies can be found in Table 2.3.

When taking a closer look on the chosen frequencies it can be noticed that E1 and E5a coincide with GPS signals L1 and L5, as well as E5b with the GLONASS G3 carrier. This is not pure coincidence but chosen for the sake of better interoperability with the competing systems. Despite this benefit, it is also accompanied by the possible problem of interference between the systems, which is avoided by using different modulation schemes for Galileo. 


\begin{tabular}{c|c} 
Component & Frequency \\
\hline Fundamental frequency & $f_{0}=10.23 \mathrm{MHz}$ \\
Carrier E1 & $154 \cdot f_{0}=1575.42 \mathrm{MHz}$ \\
Carrier E6 & $125 \cdot f_{0}=1278.75 \mathrm{MHz}$ \\
Carrier E5 & $116.5 \cdot f_{0}=1191.795 \mathrm{MHz}$ \\
Carrier E5a & $115 \cdot f_{0}=1176.45 \mathrm{MHz}$ \\
Carrier E5b & $118 \cdot f_{0}=1207.14 \mathrm{MHz}$
\end{tabular}

Table 2.3: Components and frequencies of Galileo signal

\subsection{Precise Point Positioning}

Precise Point Positioning (PPP) is a GNSS-based positioning technique which uses undifferenced single- or dual-frequency carrier phase and/or pseudorange measurements from a single receiver (Hinterberger 2016). Its main advantage is independence of additional data from (nearby) reference stations, unlike other prominent techniques such as Real Time Kinematic (RTK). Instead, precise orbit and clock information as provided by e.g. the International GNSS service (IGS) is utilized to ensure high-quality results. Using dual-frequency observations in combination with final orbit/clock solutions, centimetrelevel accuracy can be reached. This potential was first shown by Zumberge et al. (1997) and since then PPP has become one of the most promising topics in GNSS research. Huge scientific efforts have been put into certain aspects like ambiguity resolution (AR) or the correct modelling of satellite and/or receiver hardware biases. Especially AR has been the topic of a number of studies, since in classical PPP usually a real-valued constant is estimated as phase bias instead of the integer ambiguity. Overcoming this issue denotes one of the most promising means to improve the accuracy of results even further. Nevertheless, the technique still deals with a number of restrictions which currently limit its potential and usage. The most prominent one is the often longer convergence time of the solution compared to RTK. In the following a comprehensive list of advantages and disadvantages of PPP is given:

- Only a sparse global station network needed (for computation of orbit/clock information), which also implies cost-effectiveness $(+)$

- No simultaneous observations (from nearby station) required $(+)$

- No errors introduced from observations at reference stations (network effects) $(+)$

- Error sources are represented as state-space correction models and not as range 
corrections in the observation space $(+)$

- Globally available/valid corrections $(+)$

- Reduced computational efforts due to smaller data amount which also implies faster availability of the results $(+)$

- Long convergence time (-)

- Complicated observation modeling (-)

- Challenge of integer ambiguity resolution for highest accuracy (-)

- High-rate and high-quality satellite clock information required (-)

\subsubsection{Observation equations}

The observation equations for the PPP algorithm are, written separately for pseudorange observations $P_{r, \nu}^{s}$

$$
P_{r, \nu}^{s}=\rho_{r}^{s}-c \delta t^{s}+c \delta t_{r}+\delta_{i o n, \nu}+\delta_{t r o}+\epsilon_{P, \nu}
$$

and phase observations $L_{r, \nu}^{s}$

$$
L_{r, \nu}^{s}=\rho_{r}^{s}-c \delta t^{s}+c \delta t_{r}-\delta_{i o n, \nu}+\delta_{t r o}+\lambda_{\nu} B_{r, \nu}^{s}+\epsilon_{L, \nu}
$$

Here the subscript $r$ denotes a GNSS receiver, $s$ a satellite and $\nu$ the corresponding frequency. Furthermore $\rho_{r}^{s}$ denotes the geometric distance between satellite and receiver, $c$ the speed of light, $\delta t^{s}$ and $\delta t_{r}$ the clock errors of satellite and receiver, $\delta_{i o n, \nu}$ the (slant) ionospheric delay on the corresponding frequency, $\delta_{\text {tro }}$ the (slant) tropospheric delay, $\lambda_{\nu} B_{r, \nu}^{s}$ the float ambiguity for phase observations and $\epsilon_{P, \nu} / \epsilon_{L, \nu}$ are additional error terms (e.g. multipath) for the corresponding observation type.

\subsubsection{Error sources}

This section aims to give an overview on some of the different error terms contained as $\epsilon$ in Equation 2.4 and 2.5. A visual overview is given in Figure 2.2, which shows the most prominent delays/influences encountered for GNSS measurements. 


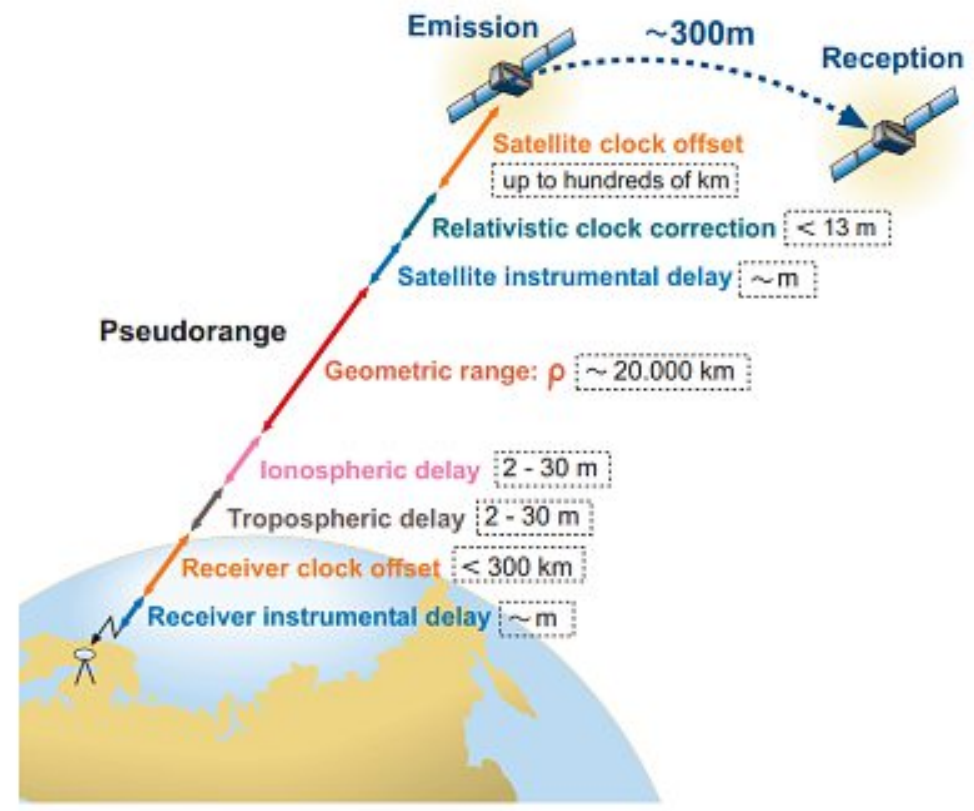

Figure 2.2: Component and error sources in pseudorange measurements, taken from Sanz Subirana et al. (2013)

\section{Relativistic effects}

Highly accurate timing capabilities are the cornerstone of the GNSS positioning technique. Therefore satellites carry atomic clocks on board, which provide highly accurate timestamps for signals leaving the satellite. Furthermore also on the receiver side, especially for high-grade (geodetic) receivers, accurate timing using precise clocks need to be ensured best as possible. The synchronisation of satellite and receiver clock denotes a fundamental requirement for GNSS positioning.

This time transfer between satellite and receiver is influenced by relativistic effects, which can be split up into two parts (as originally defined by Einstein):

- General Relativity effect: The gravitational field of the Earth influences the clock frequency differently for clocks in space and on Earth.

- Special Relativity effect: The travel speed of the satellites relative to users/receivers manifests in a clock shift (time dilatation effect).

The combined effect results in a shift of the center frequency $f_{0}$ given by:

$$
\frac{\Delta f}{f_{0}}=\frac{f-f_{0}}{f_{0}}=\frac{v^{2}}{2 c^{2}}+\frac{\Delta U}{c^{2}}=\frac{v^{2}}{2 c^{2}}+\frac{G M_{E}}{c^{2}}\left(\frac{1}{r_{s}}-\frac{1}{r_{E}}\right)
$$

$v$ denotes the satellite clock velocity relative to the ground receiver, $c$ the speed of light and $\Delta U$ the difference in gravitational potential between satellite and receiver. 
Integrating the above equation results in a clock correction of 38.6 microseconds per day (Karabatic 2011).

It is worth mentioning here that the two relativistic effects counteract each other. Due to General Relativity, clocks on board of the satellites run faster with increasing altitude (approximately 45 microseconds per day). Specific Relativity on the other hand states that the (relatively) moving clocks on board of the satellites run slower than their companions on ground. The combined effect is known as the 'factory offset' which is applied to the satellite clocks before the satellite launch.

Another related effect to be considered in the framework of high-precision applications is the Sagnac effect. It is a result of the Earth's rotation taking place while the GNSS signal is traveling from satellite to receiver. The travel time correction can be calculated as

$$
\delta t=\frac{\vec{v} \cdot\left(\overrightarrow{r_{R}}-\overrightarrow{r_{S}}\right)}{c^{2}}
$$

where $\vec{v}$ is the geocentric velocity vector of the receiver and $\overrightarrow{r_{R}}$ and $\overrightarrow{r_{S}}$ are the geocentric vectors of receiver and satellite. The correction due to the Sagnac effect can be considered software-internally in PPP processing.

\section{Multipath}

The Multipath effect occurs when a GNSS signal is reflected off an object, such as the wall of a building before it is received at the antenna. These reflected signals can distort the code correlation peak and result in a ranging error. Multipath denotes one of the dominant error sources in GNSS positioning, especially in heavily obstructed (e.g. urban) areas. This is worsened by the fact that modelling its influence on measurements is a very challenging task and complete elimination of the effect is still practically impossible for highly dynamic environments (autonomous driving, railway). However advancements in antenna design and specific filter algorithms have made it easier to recognize and correct especially Non-Line-of-sight multipath.

Multipath is a highly localized error which does not cancel through differencing and therefore must be considered as the major source of error in many high precision applications. It effects both code and carrier phase measurements, where the introduced errors can reach up to 150 meters for code observations and up to $5 \mathrm{~cm}$ for carrier phases. Moreover, multipath can prevent coordinate convergence in PPP processing. Therefore it denotes one the most challenging error sources in GNSS processing, especially for dynamic applications. 


\section{Antenna phase center offsets}

GNSS measurements refer to the phase centers of the antenna of satellite and receiver respectively. Offsets and variations in the location of the phase centers therefore directly influence the accuracy of processing results. The effects to be considered are:

- Satellite antenna phase center (APC) offset and variation: This holds for orbital information in the broadcast navigation messages. However, precise orbits instead are referred to the center of mass of the satellite, which makes a correction to the phase center necessary for precise PPP applications.

- Receiver APC offset and variation: As for the satellite antenna, the measurements are also referred to the phase center of the receiver antenna. Since this location is not a suitable reference, a point tied to the base of the antenna, denoted as antenna reference point (ARP), is used instead. Information on the antenna phase center offset between the APC and ARP normally are provided by the manufacturers or can be obtained from the IGS. Usually the receiver coordinates are referred to a monument marker or to an external bench mark. The computed coordinates in the IGS SINEX files are referred to the monument marker. The site eccentricity vector between the ARP and the monument marker is also given in the SINEX file, in up, north and east coordinates.

\section{Phase wind-up correction}

The phase wind-up effect affects only the phase measurements and depends on the mutual orientation of the satellite and the receiver antennas. Phase wind-up arises due to the nature of the GPS signals as right-hand circularly polarized radio waves. For a static receiver, like the receivers of a reference network, the wind-up effect is due to the satellite orbital motion. Usually satellites undergo only slow rotations in order to keep their solar panels pointing to the sun direction. This causes a phase variation, which is misunderstood as a range variation. However, the satellites also undergo rapid rotations in the case of eclipse and noon seasons. During such an event the rotation of the antenna can reach up to one revolution, which corresponds to an increase in the carrier phase measurement of one cycle, within less than half an hour. For doubledifference positioning on distances less than few hundreds of kilometres the carrier phase wind-up effect can be neglected. However, for longer baselines and in undifferenced point-positioning, this effect has to be taken into account. Neglecting the effect and fixing orbits and clocks will lead to positioning errors in the dm-level (Kouba and Héroux 2001). 
Following Karabatic (2011), the computation of the phase wind-up can be carried out using the formulas presented by Wu et al. (1992):

$$
\delta \phi=\operatorname{sign}(\epsilon) \arccos \left(\frac{\overrightarrow{D^{\prime}} \cdot \vec{D}}{\left|\overrightarrow{D^{\prime}}\right||\vec{D}|}\right),
$$

where $\epsilon=\vec{k} \cdot\left(\overrightarrow{D^{\prime}} \times \vec{D}\right), \vec{k}$ denotes the satellite-receiver unit vector and $\overrightarrow{D^{\prime}}, \vec{D}$ the effective dipole vectors of satellite and receiver. The latter can be derived from the satellite's $\left(\overrightarrow{x^{\prime}}, \overrightarrow{y^{\prime}}, \overrightarrow{z^{\prime}}\right)$ and receiver's unit vectors $(\vec{x}, \vec{y}, \vec{z})$ in their respective body coordinate system:

$$
\begin{gathered}
\overrightarrow{D^{\prime}}=\overrightarrow{x^{\prime}}-\vec{k}\left(\vec{k} \cdot \overrightarrow{x^{\prime}}\right)-\vec{k} \times \overrightarrow{y^{\prime}}, \\
\vec{D}=\vec{x}-\vec{k}(\vec{k} \cdot \vec{x})-\vec{k} \times \vec{y} .
\end{gathered}
$$

\subsubsection{Linear Combinations of GNSS Observations}

In GNSS data processing it is common practice to combine simultaneous observations using linear combinations (LC). Different types of combinations exist for different purposes, the most important ones are introduced in the following. In the subsequent formulas observations on two frequencies are assumed. The equations can be generalized to any pair of signals at different frequencies offered by the GNSS systems.

\section{lonosphere-free linear combination}

Undoubtedly the most important LC in GNSS processing is the lonosphere-free linear combination (IF-LC), which builds the basis for most processing strategies. It utilizes the dispersive nature of the ionosphere in order to mitigate its first-order effects (up to $99.9 \%$ ) by a combination of two-frequency carrier phase (or code) observations. For carrier phase it reads

$$
L_{I F}=\frac{1}{f_{1}^{2}-f_{2}^{2}}\left(f_{1}^{2} L_{1}-f_{2}^{2} L_{2}\right)
$$

and for pseudorange observations

$$
P_{I F}=\frac{1}{f_{1}^{2}-f_{2}^{2}}\left(f_{1}^{2} P_{1}-f_{2}^{2} P_{2}\right)
$$

Especially $L_{I F}$ denotes the common observable in (DF) data processing techniques like PPP.

However, there are also problems encountered in GNSS processing when using this LC. The two most common ones are:

- Difficult ambiguity fixing 
- Higher noise level: The IF-LC shows an increased noise level compared to the original carrier frequencies ( $\sim$ factor 3 compared to $\left.L_{1}\right)$. Further details on this are given in Section 2.3.3.

\section{Geometry-free linear combination}

As a counterpart of the IF-LC, the geometry-free LC (GF-LC or $L_{4}$ ) exposes the ionospheric delay by removing all non-dispersive parts. Therefore this LC is often used for the estimation of ionosphere models, as also in this study for the SEID algorithm (see Chapter 6). The GF-LC is formed by the difference between two measurements on different frequencies, with all non-frequency dependent errors, such as satellite, receiver clock and geometry (orbit and station coordinates) cancelling. For phase observations, the GF-LC can be formulated as:

$$
L_{G F}=L_{4}=L_{1}-L_{2}
$$

and for pseudorange observations it reads

$$
P_{G F}=P_{4}=P_{2}-P_{1}
$$

As mentioned above, the GF-LC extracts the ionospheric delay and therefore the basis of many ionospheric modelling algorithms.

\section{Wide-lane linear combination}

The Wide-lane LC $\left(L_{W L}\right)$ is primarily designed to construct an observation with a larger wavelength, which can be beneficial for cycle slip detection and ambiguity fixing. It is derived by

$$
L_{W L}=\frac{f_{1} L_{1}-f_{2} L_{2}}{f_{1}-f_{2}}
$$

\section{Narrow-lane linear combination}

The Narrow-lane LC $\left(L_{N L}\right)$ provides an observation with shorter wavelength and a lower noise level compared to the original carriers. It allows to increase precision in ambiguity fixed processing (Möller 2017).

$$
L_{N L}=\frac{f_{1} L_{1}+f_{2} L_{2}}{f_{1}+f_{2}}
$$

\section{Melbourne-Wübenna linear combination}

The Melbourne-Wübbena LC $\left(L_{M W}\right)$, proposed by Melbourne (1985), can be seen as an extension to the WL-LC. In addition to the phase observations forming the first 


\begin{tabular}{lccc} 
Observable & Wavelength & Noise level & Multipath level \\
\hline$P_{1,2}$ & $19.0 / 24.2 \mathrm{~cm}$ & $\sigma_{\text {code }}= \pm 15 \mathrm{~cm}$ & $m p_{\text {code }}= \pm 150 \mathrm{~m}$ \\
$L_{1}$ & $19.0 \mathrm{~cm}$ & $\sigma_{L 1}= \pm 1-2 m m$ & $m p_{L 1}= \pm 5 \mathrm{~cm}$ \\
$L_{2}$ & $24.2 \mathrm{~cm}$ & $1.3 \cdot \sigma_{L 1}$ & $1.3 \cdot m p_{L 1}$ \\
$L_{I F}$ & $0.6 \mathrm{~cm}$ & $3.2 \cdot \sigma_{L 1}$ & $4.5 \cdot m p_{L 1}$ \\
$L_{G F}$ & $\infty$ & $1.6 \cdot \sigma_{L 1}$ & $2.3 \cdot m p_{L 1}$ \\
$L_{W L}$ & $86.2 \mathrm{~cm}$ & $6.4 \cdot \sigma_{L 1}$ & $9.1 \cdot m p_{L 1}$ \\
$L_{N L}$ & $10.7 \mathrm{~cm}$ & $0.8 \cdot \sigma_{L 1}$ & $1.1 \cdot m p_{L 1}$ \\
$L_{M W}$ & $86.2 \mathrm{~cm}$ & $\sim \sigma_{\text {code }}$ & $9.1 \cdot m p_{L 1}$
\end{tabular}

Table 2.4: Overview on noise and multipath characteristics of GNSS observables, most information taken from Möller (2017).

part of the equation, code observations are used in the second part to mitigate the ionospheric effects. Thus, the $L_{M W}$ is free from ionospheric delays, geometry, clock, and tropospheric effects. The disadvantage of the inclusion of code observations in the LC is the significant increase of the observation noise and the multipath effects. However, in the future, advanced code modulations with significantly improved noise characteristics will considerably reduce the noise of this combination. The Melbourne-Wübbena LC is formed as follows:

$$
L_{M W}=\frac{1}{f_{1}^{2}-f_{2}^{2}}\left(f_{1} L_{1}-f_{2} L_{2}\right)-\frac{1}{f_{1}+f_{2}}\left(f_{1} P_{1}+f_{2} P_{2}\right)
$$

Expect for multipath and measurement noise, $L_{M W}$ covers solely the wide-lane ambiguities. Therefore it can be used for cycle slip detection, which is exploited in the pre-processing of reference station data for the SEID algorithm as described in Chapter 6. In the case of low multipath, low code noise and real-value widelane ambiguities, the $L_{M W}$ is directly used for the resolution of wide-lane ambiguities (Schönemann 2014).

A number of other useful LCs can be found in GNSS literature, e.g in Glaner (2017).

\section{Noise characteristics}

As already seen before for the IF-LC, some LCs show undesirable effects by introducing a higher noise level in the data processing compared to observations on the original carrier frequencies $\left(L_{1} / P_{1}\right.$ and $\left.L_{2} / P_{2}\right)$. Therefore an overview on the noise characteristics of different combinations of GNSS observations is given in Table 2.4 . 


\subsubsection{Integer ambiguity resolution}

Although integer ambiguity estimation is not carried out within this study it should still be mentioned here as a key ingredient for high-accuracy applications. If centimetreaccuracy level is required for the position solution, fixing of the phase ambiguities to integer values is essential. For PPP processing, integer resolution is achieved by applying improved satellite products, where phase delays are separated from the integer ambiguities (Hinterberger 2016). In general two approaches can be distinguished:

- Ge et al. (2008): Decomposition of undifferenced ionosphere-free ambiguities into wide-lane and narrow-lane part.

- Laurichesse et al. (2009): Undifferenced ambiguities were directly fixed to integers.

For a detailed discussion of those approaches and further considerations on integer ambiguity resolution see e.g. Hinterberger (2016) or Glaner et al. (2020).

\subsubsection{Kinematic PPP}

Kinematic PPP is a promising processing technique for a number of applications which do not allow for the assumption of a static rover position, as deployed in (standard) static PPP. Kinematic GPS PPP was first investigated by Kouba and Héroux (2001) and found well suited for positioning of moving vehicles as needed e.g. in autonomous driving or agricultural applications. Although reachable accuracy is still lower than for techniques like RTK positioning, the simplicity of the approach in terms of data requirements still is a promising feature for a lot of users.

Over the last decade, different applications and processing strategies (e.g. concerning GNSS systems or orbit/clock products used) have been investigated.

Alkan et al. (2017) used kinematic GPS/GLONASS PPP for marine applications and found an accuracy level of centimetre- to decimetre-level even in challenging conditions as well as improvements in solution availability through the usage of GLONASS observations. Katsigianni et al. (2019) examined the performance of the Galileo-only PPP and PPP-AR solutions in post-processing kinematic mode. Results indicate millimetreto centimetre-level accuracy of the PPP solution, especially for PPP-AR processing. Yu and Gao (2017) studied the benefit of using multi-GNSS observations and found an significant enhancement in availability and accuracy of the positioning solution. Moreover they also found an increase in convergence performance for all three coordinate components. 


\section{Chapter 3}

\section{Physics of the atmosphere}

This section aims to give an overview on the principles and parameters, which are exploited to draw conclusions on the current state of the atmosphere. In order to get a first glance of the medium that is investigated in the course of this study, some general information is provided here.

\subsection{General overview}

The term Atmosphere refers to the gaseous envelope of the Earth or any other celestial body (Möller 2017). The atmosphere of the Earth is the result of volcanic fumigation approximately four billion years ago. Without this formation process, life would not have been able to establish itself on the planet due to a number of reasons. This layer (or at least its bottom end) is where weather and climatic phenomena take place, which are of larger interest than ever in a changing climate as experienced today.

In the following, some basic information on optical properties, chemical composition and vertical structure of the atmosphere is summarized. This short overview is strongly based on Wallace and Hobbs (2006), who provide a detailed description not only on these introduction topics but on all major fields of atmospheric research in general.

\subsubsection{Optical properties and energy budget}

The optical properties of the Earth's atmosphere can briefly be summarized by two main properties:

- Transparency for incoming solar (short-wave) radiation

- Opaqueness for outgoing (long-wave) radiation emitted by the Earth's surface 


\begin{tabular}{lc} 
Process & Radiation fraction \\
\hline Solar irradiation (short-wave) absorbed at surface & $+161 \mathrm{~W} / \mathrm{m}^{2}$ \\
Thermal emission by the Earth's surface & $-396 \mathrm{~W} / \mathrm{m}^{2}$ \\
Long-wave downward radiation (e.g. from clouds) & $+333 \mathrm{~W} / \mathrm{m}^{2}$ \\
\hline Net radiation & $+98 \mathrm{~W} / \mathrm{m}^{2}$
\end{tabular}

Table 3.1: Net radiation budget of the Earth, Möller (2017)

These properties manifest themselves in a warming of the Earth's surface compared to temperatures to be expected from classical (blackbody) radiative transfer theory. This behaviour is what is commonly referred to as the Greenhouse Effect.

Considerations on the energy budget of the whole Earth system are therefore closely linked to these properties. The derivation of the systems energy budget relies on the concept of blackbody radiation. The term blackbody describes a surface where all incoming energy is absorbed. Its flux density (i.e. irradiance) $F$ is given by the StefanBoltzmann law

$$
F=\sigma T^{4}
$$

with $\sigma=5.67 \cdot 10^{-8} \mathrm{Wm}^{-2} \mathrm{~K}^{-4}$ being the Stefan-Boltzmann constant and $T$ the temperature of the surface.

Inserting the relevant parameters for the Earth gives the net radiation budget outlined in Table 3.1. The remaining excess of $98 \mathrm{~W} / \mathrm{m}^{2}$ in the budget is compensated by nonradiative processes like sensible (e.g. convection) or latent heat fluxes (e.g. melting processes).

\subsubsection{Atmospheric composition}

The Earth's atmosphere is composed of a mixture of different gases. The dominant ones include hydrogen $(\mathrm{H})$, carbon dioxide $\left(\mathrm{CO}_{2}\right)$ and methane $\left(\mathrm{CH}_{4}\right)$. Together with ozone $\left(\mathrm{O}_{3}\right)$, they denote the most important greenhouse gases. This means that their molecular structure allows them to effectively trap outgoing long-wave radiation, and therefore cause the greenhouse effect.

The dominant gases in terms of concentration in the atmosphere are diatomic nitrogen $\left(\mathrm{N}_{2}\right)$ and oxygen $\left(\mathrm{O}_{2}\right)$. Together they account for over $99 \%$ of fractional concentration by volume. A detailed overview of the major constituents of the atmosphere can be found in Table 3.2 . 


\begin{tabular}{lcc} 
Constituent & Molecular weight & Fractional concentration by volume \\
\hline Nitrogen $\left(\mathrm{N}_{2}\right)$ & 28.013 & $78.08 \%$ \\
Oxygen $\left(\mathrm{O}_{2}\right)$ & 32.000 & $20.95 \%$ \\
Argon $(\mathrm{Ar})$ & 39.95 & $0.93 \%$ \\
Water vapour $\left(\mathrm{H}_{\mathbf{2}} \boldsymbol{O}\right)$ & 18.02 & $0-5 \%$ \\
Carbon dioxide $\left(\mathrm{CO}_{\mathbf{2}}\right)$ & 44.01 & $380 \mathrm{ppm}$ \\
Neon $(\mathrm{Ne})$ & 20.18 & $18 \mathrm{ppm}$ \\
Helium $(\mathrm{He})$ & 4.00 & $5 \mathrm{ppm}$ \\
Methane $\left(\mathrm{CH}_{\mathbf{4}}\right)$ & 16.04 & $1.75 \mathrm{ppm}$ \\
Nitrous oxide $\left(\mathrm{N}_{\mathbf{2}} \boldsymbol{O}\right)$ & 56.03 & $0.3 \mathrm{ppm}$ \\
Ozone $\left(\boldsymbol{O}_{\mathbf{3}}\right)$ & 56.03 & $0.3 \mathrm{ppm}$
\end{tabular}

Table 3.2: Fractional concentrations of gaseous atmospheric constituents with respect to dry air, with important greenhouse gases marked bold. Taken from Wallace and Hobbs (2006)

\subsection{Vertical structure and layers of the atmosphere}

This section discusses the vertical distribution of three essential atmospheric variables: pressure, temperature and water vapour. Therefore the concept of hydrostatic balance is introduced which allows finding the relationship with air density and atmospheric pressure. Based on the described distributions (mainly on that of temperature), distinctive layers are introduced in which the atmosphere is commonly divided.

Due to its importance for this study, water vapour and its distribution and characteristics are discussed separately in Section 3.3.

\subsubsection{Hydrostatic balance}

Assuming a static atmosphere, pressure on every vertical level only depends on the weight of the (fluid) layers above it. This is what is commonly referred to as hydrostatic balance. Imagine a vertical column of air as shown in Figure 3.1. It is obvious that both pressure $p$ and density $\rho$ are (at least) dependent on height $z$. For a small change in height $\delta z$ the corresponding pressure change $\delta p$ is

$$
\delta p=\frac{\partial p}{\partial z} \delta z
$$

The assumption of a static (i.e not accelerating) vertical column implies that the net force on it must be zero. This net force consists of the following vertical forces (negative sign indicates downward force) 


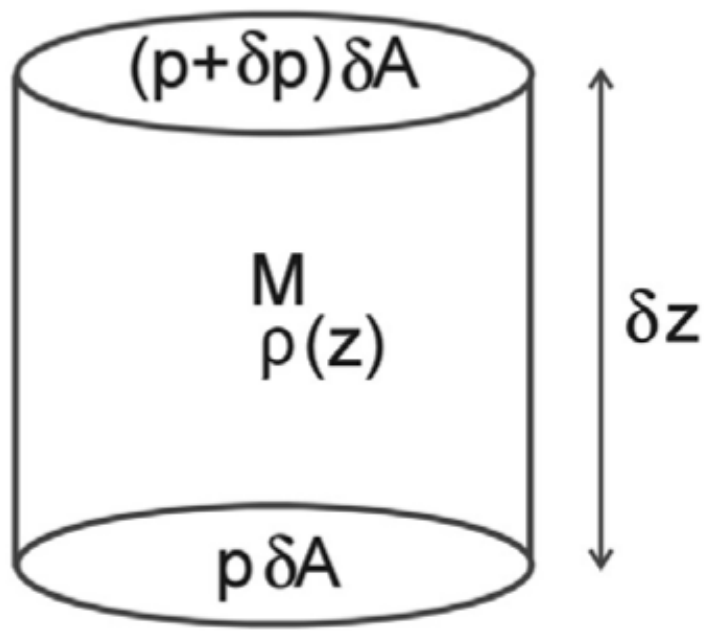

Figure 3.1: A vertical column of air of density $\rho$, horizontal cross-sectional area $\delta \mathrm{A}$, height $\delta \mathbf{z}$ and mass $\mathbf{M}=\rho \delta \mathrm{A} \delta \mathrm{z}$. The pressure on the lower surface is $\mathrm{p}$, the pressure on the upper surface is $\mathrm{p}+\delta \mathrm{p}$.

- gravitational force $F_{g}=-g M=-g \rho \delta A \delta z$,

- pressure force acting at the top face, $F_{T}=-(p+\delta p) \delta A$, and

- pressure force acting at the bottom face, $F_{B}=p \delta A$.

Setting the net force $F_{g}+F_{T}+F_{B}=0$ one obtains $\delta p+g \rho \delta z=0$ and by using Equation 3.2 finds

$$
\frac{\partial p}{\partial z}+g \rho=0
$$

the equation of hydrostatic balance. It describes the decrease in atmospheric pressure with height, i.e with decreasing weight of the overlying layers. The assumption of negligible vertical acceleration of the air column is an appropriate one in most circumstances. Exceptions are small-scale phenomena like convection, tornados, or turbulence in atmospheric boundary layer (ABL).

\subsubsection{Vertical structure of pressure/density}

An important information Equation 3.3 withholds is the actual value of $p(z)$. Therefore the equation of state of air is used to rewrite Equation 3.3 to

$$
\frac{\partial p}{\partial z}=-\frac{g p}{R T}
$$

where $T$ is the atmospheric temperature and $\mathrm{R}=8314.459 \mathrm{~J} /(\mathrm{kmolK})$ the ideal gas constant. 
For an isothermal atmosphere $\left(T=T_{0}=\right.$ const. $)$ Equation 3.4 reads

$$
\frac{\partial p}{\partial z}=-\frac{g p}{R T_{0}}=-\frac{p}{H}
$$

where the constant $H=R T_{0} / g$ is called the scale height. The resulting pressure $p$ at height $z$ then is

$$
p(z)=p_{s} e^{\left(-\frac{z}{H}\right)},
$$

where $p_{s}=p(z=0)$ is the pressure at the surface $z=0$. Equation 3.6 describes the exponential decrease of pressure with height. The isothermal assumption delivers quite reasonable results for the structure which is actually observed.

In the more realistic case of an non-isothermal atmosphere, where temperature $T(z)$ and scale height $H(z)$ are a function of height too, pressure $p(z)$ is found as

$$
p(z)=p_{s} e^{\left(-\int_{0}^{z} \frac{d z}{H(z)}\right)} .
$$

\subsubsection{Vertical distribution of temperature}

Temperature profiles show large variations especially in vertical direction throughout the whole atmosphere. The vertical structure however, is somehow similar everywhere and thus it is convenient to introduce a 'typical' profile. For the sake of simplicity only this profile will be introduced here. However, it should be mentioned that both vertical and horizontal deviations from this profile exist. The vertical ones for example are connected to the meteorological term of stability, especially in the ABL.

The typical mid-latitude vertical temperature profile is shown in Figure 3.2. It confirms the high variations in the profile and it's rather complicated nature. The extreme values of the profile can be used to distinguish between four layers, which represent different temperature tendencies and therefore different physical conditions and properties. These layers are introduced in detail in the next section.

\subsubsection{Atmospheric layers}

Starting from top to bottom, the first layer of the atmosphere is called Thermosphere. This is the region where highly energetic UV radiation is absorbed, mainly by $\mathrm{O}_{2}$ and $\mathrm{CO}_{2}$. At the top (border to free space), temperature is very high and decreases down to $90 \mathrm{~km}$ height where it stays fairly constant down to the Mesopause.

Below, temperature increases again throughout the whole Mesosphere down to the Stratopause at $50 \mathrm{~km}$ height. This maximum is directly connected with the maximum in the absorption of mid-wavelength UV radiation by stratospheric ozone $\left(\mathrm{O}_{3}\right)$. 


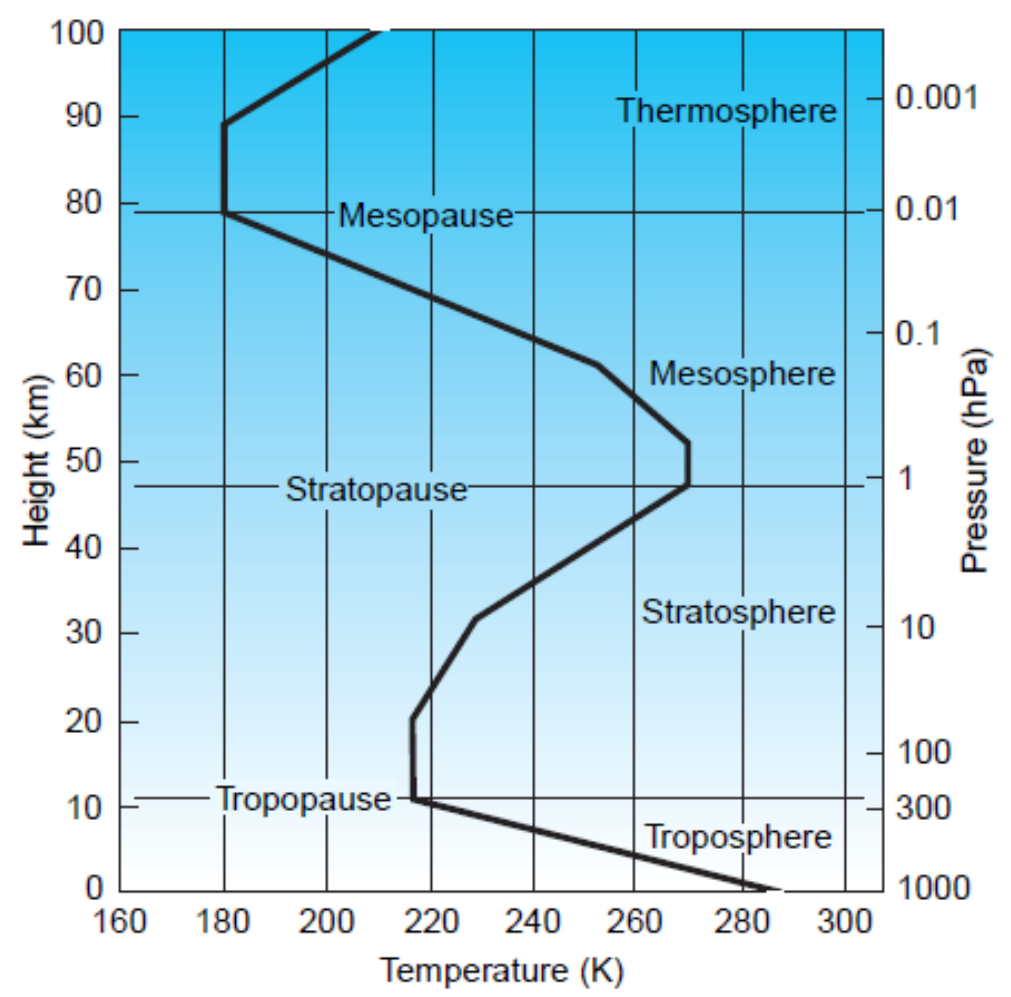

Figure 3.2: Typical atmospheric temperature profile from U.S. standard atmosphere, taken from Wallace and Hobbs (2006)

However the maximum in the ozone concentration is observed at lower heights (20-30 $\mathrm{km}$ ) in the Stratosphere, caused by the fact that the ozone is very opaque to UV. In principle the whole existence of the layer is due to the existence of this high ozone concentration. The air is typically very dry and temperature decreases throughout the layer down to the Tropopause.

The tropopause is located between 8-15 km, the actual height depends both on latitude and season. It marks the typical upper height limit for existence of water in all forms (and therefore e.g. of clouds) in the atmosphere. The layer from there down to the surface of the Earth is called Troposphere. Here, temperature increases again by typically 70-80 Kelvin from the tropopause down to the surface. Practically all physical phenomena that we group under the term 'weather' are taking place within this layer. This is directly linked to the fact that, as already mentioned above, nearly all water is concentrated here. 


\subsection{Water vapour in the atmosphere}

A constituent of special interest for this study is water vapour. Although it only accounts for about $0.25 \%$ of the atmospheric mass, it denotes a key component of the system due to its high spatial and temporal variability and its role as a greenhouse gas. Water vapour is often concentrated very locally in the atmosphere due to to the fact that it remains there only about $8-10$ days. This residence time is based on the total mass of water in the atmosphere (around $30 \mathrm{~kg} / \mathrm{m}^{2}$ which corresponds to a global water layer of around $3 \mathrm{~cm}$ ) and a mean rainfall rate of roughly $0.3 \mathrm{~cm} /$ day (Wallace and Hobbs 2006). The amount of water vapour which can be conserved in the atmosphere at a certain temperature is given by the Clausius-Clapeyron relation

$$
\frac{1}{e_{s}} \frac{\Delta e_{s}}{\Delta T} \sim \frac{L_{v} M_{w}}{1000 R^{*} T^{2}} .
$$

Thereby $e_{s}$ represents the water vapour pressure at saturation level, $T$ the temperature, $L_{v}$ the latent heat of vaporization, $M_{w}$ the molecular weight of $\mathrm{H}_{2} \mathrm{O}$ and $\mathrm{R}^{*}$ the universal gas constant.

This relation has a massive impact on the evolution of climate change, as it implies that with a rising global temperature the atmosphere is capable of capturing more water vapour and therefore also a larger amount of energy. This will again amplify the temperature increase through the greenhouse effect and is expected to also lead to increasing activity in all kinds of severe weather events related to the precipitation (tropical storms, floods, extreme precipitation events, ...).

In general, water appears in the troposphere in three different forms:

- solid: snow, hail, graupel, ice

- liquid: rain, clouds, fog

- gaseous: water vapour

As mentioned earlier, water vapour shows strong variations in spatial and temporal directions. This is in complete contrast to dry air which has no significant variation in composition with latitude or height (Smith and Weintraub 1953). These strong variations also make it difficult to measure or model its local or global distribution. Typical instruments to do so are hygrometers, with whom measurements at surface as well as vertical profiles from radiosondes can be derived. It becomes obvious that the horizontal (and temporal) resolution of those measurements is rather poor and definitely not sufficient to describe such strong variability. 
Over the last decades more and more possibilities to measure water vapour (or related parameters) indirectly with satellites have been investigated. Nowadays a number of satellite missions carry suitable instruments like interferometers, operating from optical to microwave regions. Although originally not designed for such purposes, GNSS can also be added to this list. This fact has given rise to a new, cost-effective and all-weather operating source of observations.

\subsubsection{Water vapour measures}

Amounts of water vapour are usually measured as a fraction of moist air compared to dry air. In the following, some measures which can reflect the water vapour content of the atmosphere are introduced.

\section{Mixing ratio}

One possible measure for the water content of air is the mixing ratio $w$. It is defined by the quotient of water vapour mass per unit mass of dry air

$$
w=\frac{m_{v}}{m_{d}}=\frac{\rho_{v}}{\rho_{d}},
$$

where $m_{v}, m_{d}$ denote the mass and $\rho_{v}, \rho_{d}$ the density of water vapour and dry air. The partial pressure of water vapor $e$ and dry air $P_{d}$ can be calculated by applying the equation of state

$$
\begin{gathered}
e=\rho_{v} R_{v} T, \\
P_{d}=\rho_{d} R_{d} T .
\end{gathered}
$$

Here $R_{v}=287.06 \pm 0.01 \mathrm{~J} \mathrm{~kg}^{-1} \mathrm{~K}^{-1}$ and $R_{d}=461.525 \pm 0.003 \mathrm{~J} \mathrm{~kg}^{-1} \mathrm{~K}^{-1}$ denote the specific gas constants of water vapour and dry air. By using Equations 3.10 and 3.11 , an alternative formulation for the mixing ratio can be found:

$$
w=\frac{e / R_{v} T}{(P-e) / R_{d} T}=\epsilon \frac{e}{P-e} \approx \epsilon \frac{e}{P},
$$

where $\epsilon=R_{d} / R_{v}=0.622$. It should be mentioned here that the approximation in Equation 3.12 is convenient because the partial pressure typically only accounts for about $1 \%$ of the total pressure.

\section{Specific humidity}

As an alternative to the mixing ratio, the measure of specific humidity $q$ can be used:

$$
q=\frac{\rho_{v}}{\rho_{m}} \approx w .
$$


Here the density of moist air (i.e the mixture of dry air and water vapor) $\rho_{m}$ is used and therefore $q$ is approximately equal to $w$.

One can also apply the equation of state for moist air which leads to

$$
P=\rho_{m} R_{d} T_{v},
$$

where $T_{v}$ is the virtual temperature

$$
T_{v}=(1+0.61 w) T
$$

given in Kelvin. It denotes the temperature that dry air at pressure $P$ would have when its density would equal that of moist air at temperature $T$, pressure $P$, and mixing ratio $w$ (Haltiner and Martin 1957). With Equation 3.15 one can account for the presence of moisture in the air.

\section{Relative humidity}

Finally, the humidity contained in air can also be expressed in relative terms to a reference state. This is referred to as relative humidity. It represents the ratio (expressed in percentage) of the actual mixing ratio $w$ to the so-called saturation mixing ratio $w_{s}$ with respect to a plane water surface (Wallace and Hobbs 2006), where the later denotes the ratio of masses between saturated and dry air. The expression for relative humidity reads

$$
R H=100 \frac{w}{w_{s}} \sim 100 \frac{e}{e_{s}},
$$

where $w$ is the actual and $w_{s}$ the saturation mixing ratio. $e$ and $e_{s}$ are the corresponding water vapour pressures. 


\subsection{Precipitation}

In meteorology, the term precipitation describes any product of the condensation of atmospheric water vapour that falls under gravity from clouds. The main forms are rain, snow, graupel and hail.

Precipitation occurs when local moist air cools, becomes saturated with water vapor and is no longer able to maintain its level of water vapor in gaseous form. Processes which can lead to this cooling are:

- an airmass cools adiabatically through rising motion (e.g. lifting on mountain ranges) or

- an airmass cools through radiative processes, or ground contact with cold terrain.

Therefore, it can originate from a number of different atmospheric processes which define its type. The three most important types are:

1. Cyclonic precipitation

2. Convective precipitation

3. Orographic precipitation

Cyclonic (or frontal) precipitation is the result of frontal systems associated with extratropical cyclones, which form when warm, tropical air meets cooler air. Air masses of different densities (moisture and temperature characteristics) meet, and warmer air is forced to rise. This creates an effect of saturation and, if conditions are favourable, causes precipitation.

Convective precipitation occurs when air rises vertically through the mechanism of convection, i.e. rising of heated air and cloud formation within a conditionally unstable atmosphere. Convective precipitation is typically a very local phenomenon, lasting over just a short time but usually at high intensity.

Orographic precipitation is somehow similar to the cyclonic type, except that the rising motion is caused by an orographic obstacle (e.g. a mountain ridge).

Figure 3.3 shows a typical climatology of precipitation derived from different data sources (reanalysis data, observations) for the period 1981-2010. The typical maxima are observed in the tropics, where large-scale convection processes lead to huge amounts of rainfall. These processes are strongly connected to significant patterns in global circulation, like the so-called Hadley circulation which brings air masses towards 

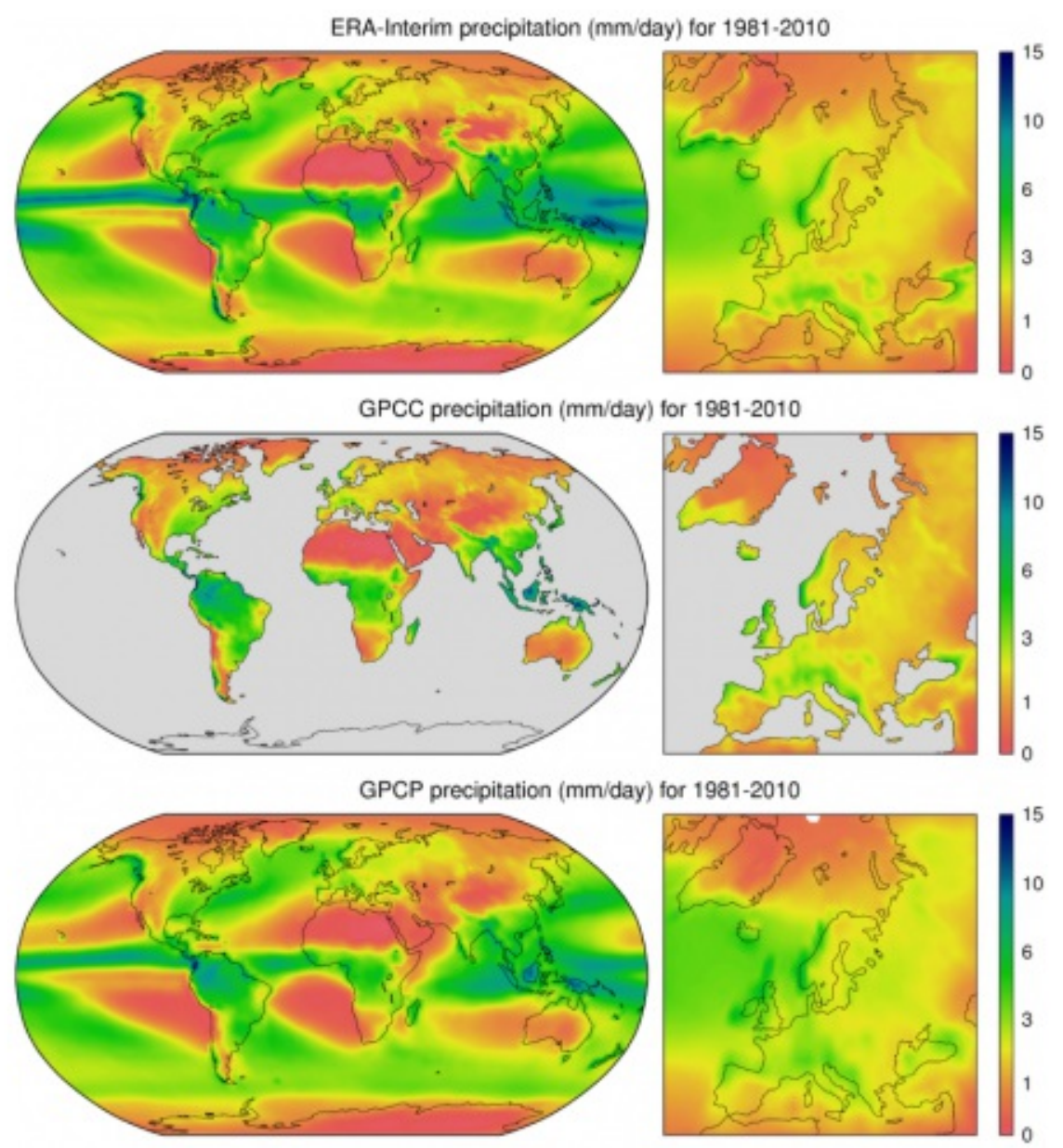

Figure 3.3: Climatological average precipitation rate $(\mathrm{mm} /$ day $)$ for 1981-2010 from ERA-Interim, Global Precipitation Climatology Centre(GPCC), Global Precipitation Climatology Project (GPCP) and E-OBS (European Observations) taken from https://climate.copernicus.eu/ monthly-summaries-precipitation-relative-humidity-and-soil-moisture

the equator and therefore also causes the constantly east-directed trade winds (Passat winds).

\subsubsection{Numerical modelling of precipitation}

Quantitative precipitation forecasting (QPF) has been a major challenge in the framework of NWP over the last years. Still, especially cases of convective precipitation in summer remain a problematic topic in this scientific area. The main problem is attached to the fact that parametrizations of deep convection used in NWP models are not able to reproduce convective systems in needed detail, as outlined by Wulfmeyer et al. (2008). Improvements can be achieved by using non-hydrostatic convection-permitting models 
and increasing horizontal resolution as shown by e.g. Ducrocq et al. (2002). Performance of models using parametrizations for deep convection still perform poor in simulating heavy and highly localized precipitation and therefore high resolution to explicitly resolve these processes is needed. This holds especially for complex terrain, where much more small-scale structures have to be resolved.

Of major interest for QPF are the mesoscale initial conditions and in particular, the initial moisture field. This is where GNSS data can provide valuable aid for models through DA. Lots of efforts have been made by international NWP centres investigating optimal combinations of observations and model output for DA systems. Nowadays these highresolution systems, based on non-hydrostatic convection resolving models with rapid update cycles, are operational in several meteorological centres (Yan et al. 2009). 


\title{
Chapter 4
}

\section{Signal propagation in the atmosphere}

\begin{abstract}
A GNSS signal recorded at the Earth's surface has to travel through the atmosphere on the way from satellite to receiver. It's concrete path and velocity is thereby determined by multiple physical principles and parameters. This chapter introduces the physical laws determining signal propagation, distinctive layers and their characteristics and how the influences of the atmosphere on the GNSS signal can be modelled.
\end{abstract}

\subsection{Maxwell equations}

In 1862, J. C. Maxwell introduced a set of equations which describe the propagation of electromagnetic waves, the so-called Maxwell equations (Jackson 1999). The general Maxwell equations read

$$
\begin{aligned}
\nabla \cdot \mathbf{E} & =\frac{\varrho}{\epsilon} \\
\nabla \times \mathbf{E} & =-\frac{\partial \mathbf{B}}{\partial t} \\
\nabla \cdot \mathbf{B} & =0 \\
\nabla \times \mathbf{B} & =\mu J+\mu \epsilon \frac{\partial \mathbf{E}}{\partial t}
\end{aligned}
$$

where $E$ is the electric field, $\varrho$ the total charge and $\epsilon$ the electric permittivity of the medium, $B$ the magnetic field, $\mu$ the permeability and $J=\kappa \cdot E$ the current electric density with $\kappa$ as the electric conductivity. In a non-conducting medium like the troposphere 
$\kappa$ and $\varrho$ are zero and Equations 4.1 become:

$$
\begin{aligned}
\nabla \cdot(\epsilon \mathbf{E}) & =0 \\
\nabla \times \mathbf{E} & =-\frac{\partial \mathbf{B}}{\partial t} \\
\nabla \cdot \mathbf{B} & =0 \\
\nabla \times \mathbf{B} & =\mu \epsilon \frac{\partial \mathbf{E}}{\partial t},
\end{aligned}
$$

Assuming only small spatial and temporal variations in $\mu$ and $\epsilon$, one can combine these equations into a wave equation for the electric field (Böhm 2004).

$$
\nabla^{2} \mathbf{E}=\mu \epsilon \frac{\partial^{2} \mathbf{E}}{\partial t^{2}}=\frac{n^{2}}{c^{2}} \frac{\partial^{2} \mathbf{E}}{\partial t^{2}}
$$

where $c=1 / \sqrt{\epsilon_{0} \mu_{0}}$ denotes the speed of light in vacuum and $n$ the refractive index. This refractive index $n$ is the key parameter to describe the propagation of an electromagnetic signal through the atmosphere. In the troposphere it is very close to one, and therefore it is good practice to use to refractivity $N$ instead, which is defined by

$$
N=(n-1) \cdot 10^{6}
$$

In general $N$ is a complex number and can be separated into three parts

$$
N=N_{0}+N^{\prime}(\nu)-i N^{\prime \prime}(\nu)
$$

The real part consists of non-dispersive part $N_{0}$ and a dispersive part $N^{\prime}(\nu)$. It causes refraction and the propagation delay of electromagnetic signals. On the other hand, the imaginary part $i N^{\prime \prime}(\nu)$ accounts for absorption and is directly related to the absorption coefficient $\alpha$

$$
\alpha(\nu)=10^{-6} \frac{4 \pi \nu N^{\prime \prime}(\nu)}{c} .
$$

While $\alpha$ is important in many other remote sensing techniques, it is typically not relevant for space geodetic measurements like GNSS or VLBI, where the travel time of the signal is observed. This is due to the fact that absorption does not affect the propagation delay of the signal.

\subsection{Path delays}

As already indicated in the last section, electromagnetic signals are delayed due to their travel through the Earth's atmosphere. When investigating the different atmospheric phenomena and properties which lead to this behaviour it is reasonable to separate the atmosphere into (at least) two parts, the ionosphere and the troposphere. 


\subsubsection{Ionospheric delay}

The ionosphere is defined as the ionized part of the atmosphere, which extends from about 60 to $1000 \mathrm{~km}$ height above ground. The ionization of particles is caused by incoming solar radiation, leading to a large number of free electrons in the layer. The whole ionospheric layer is typically split into different parts which are indicated by Figure 4.1. How many layers can be distinguished varies from day (four) to nighttime (two), again related to the amount of solar radiation. These are: $D, E, F 1$ and $F 2$, the

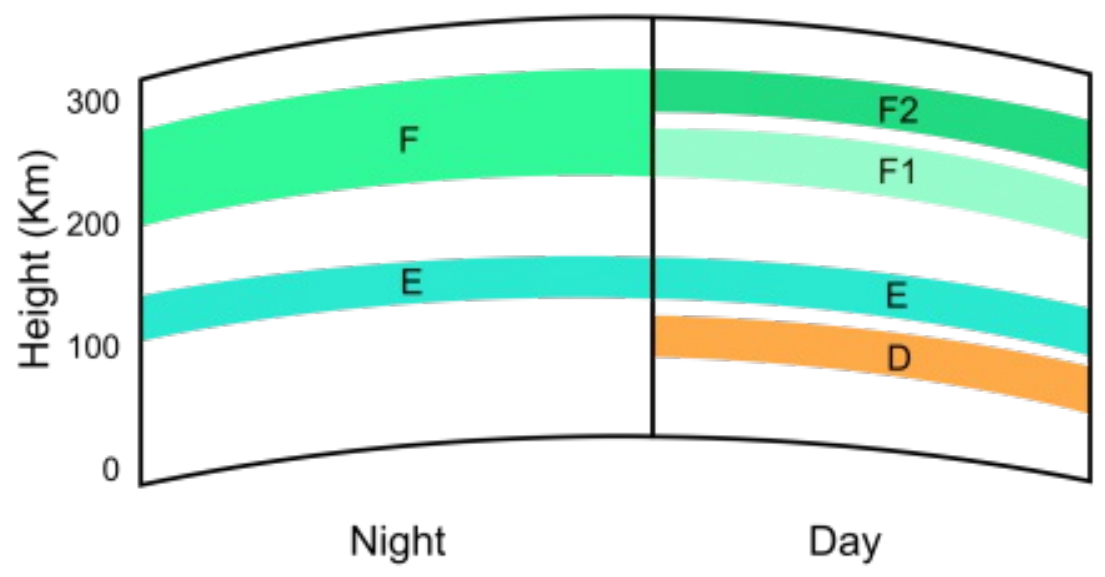

Figure 4.1: lonospheric layers present at day- and nighttime and their respective heights, taken from https://commons. wikimedia.org/w/index.php?curid $=7009115$

latter being largely responsible for the ionospheric effects which typically affect GNSS applications. Typical vertical profiles of electron density for Vienna and different seasons (January and July) at day and night-time are shown in Figure 4.2. Large differences for day and night-time are indicated, which are amplified in the winter season. The peaks of the profiles around $300 \mathrm{~km}$ are all located in the F/F2 region (compare to Figure 4.1), explaining its importance for GNSS applications which was outlined before. The electron density is normally expressed in terms of Total Electron Content (TEC) units, which is denoted as the electron content $N_{e}$ along the ray path from satellite (S) to receiver (R)

$$
T E C=\int_{S}^{R} N_{e} d s .
$$

In general we distinguish between vertical total electron content (VTEC) and TEC along the ray path, slant total electron content (STEC). Since TEC values are normally provided as VTEC, a mapping function has to be used to gain the STEC for a certain signal 


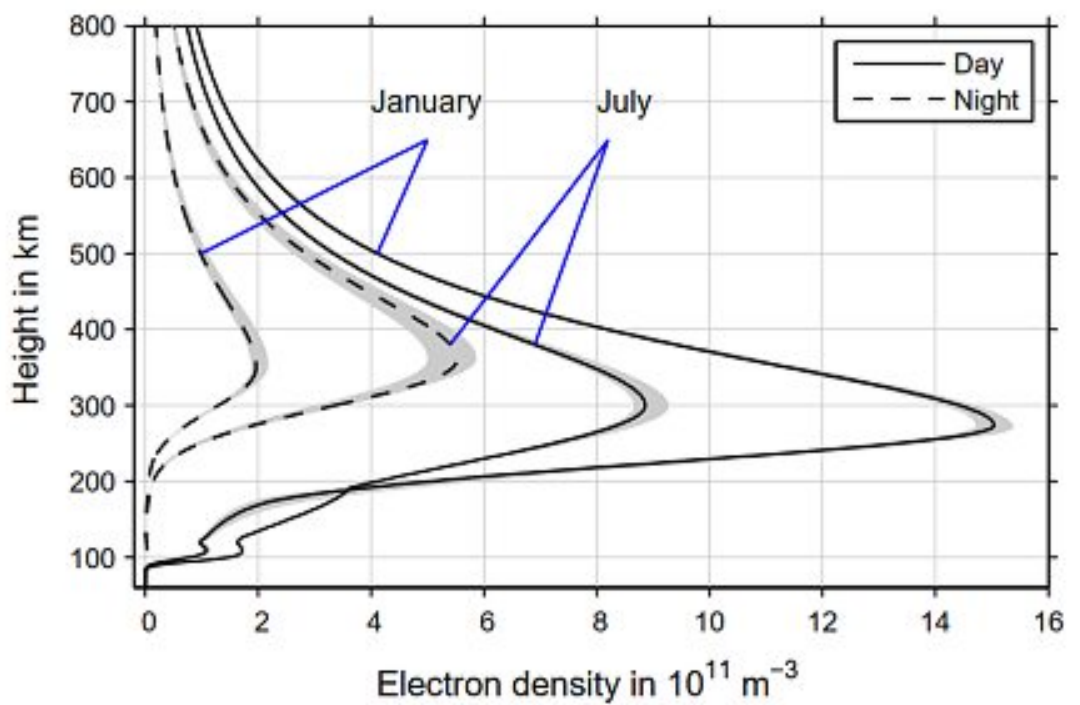

Figure 4.2: Ionospheric electron density profiles computed from the International Reference lonosphere (IRI) model for Vienna in 2017, taken from Magnet (2019).

from a satellite

$$
S T E C=\frac{V T E C}{\cos z^{\prime}}
$$

with

$$
\cos z^{\prime}=\sqrt{1-\frac{R^{2} \sin ^{2} z}{(R+H)^{2}}},
$$

where $z$ is the elevation angle of the satellite, $R$ is the mean radius of the Earth and $H$ is the effective ionospheric height, i.e. the height of the layer assumed to contain all electrons.

TEC values understandably show a strong dependence on time of day as well as elevation of the satellite. Furthermore due to influence of the Earth's magnetic field, it is also a function of latitude.

\section{Correcting for ionospheric delays}

For GNSS signals, the ionosphere is a dispersive medium, meaning the propagation velocity is frequency-dependent. Therefore the formulations of the ionospheric delay for phase and group measurements read

$$
\begin{array}{r}
\Delta \rho_{i o n, p h}=-\frac{40.31}{f^{2}} \int N_{e} d s=-\frac{40.31}{f^{2}} \text { STEC } \\
\Delta \rho_{i o n, g r}=\frac{40.31}{f^{2}} \int N_{e} d s=\frac{40.31}{f^{2}} \text { STEC },
\end{array}
$$

where $f$ is the frequency of the signal, $N_{e}$ is the number of free electrons, $d s$ is the signal path and STEC denotes the slant total electron content along the ray path of 


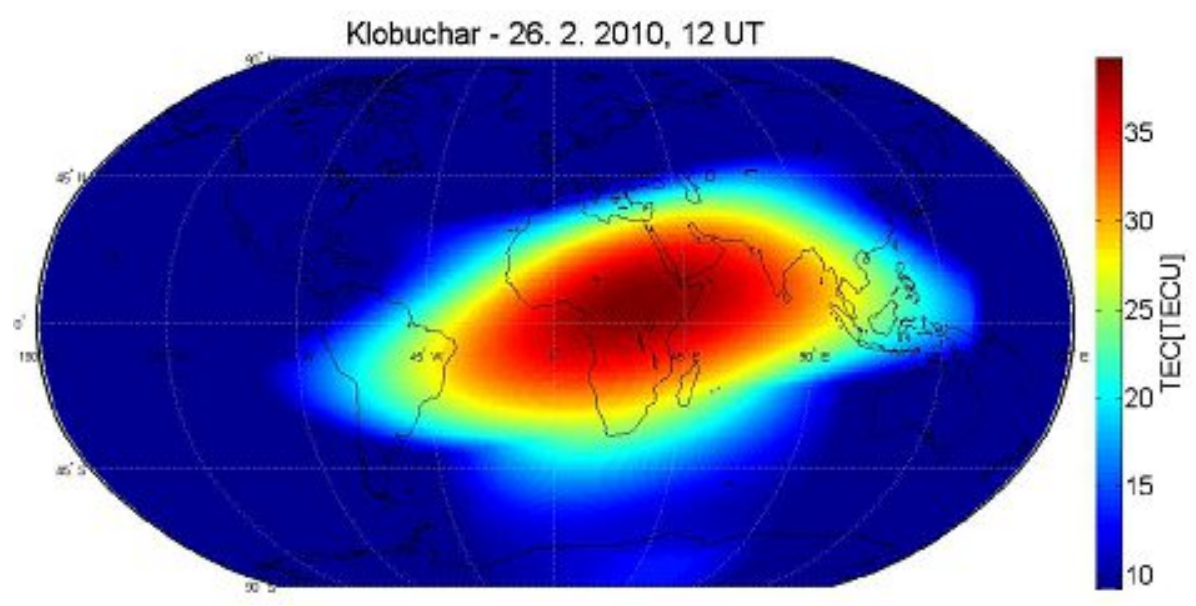

Figure 4.3: VTEC map for 26.02.2010, 12 UTC derived using the Klobuchar model. Typical maximums in VTEC are visible at low latitudes. Taken from Najman and Kos (2014)

the signal. The constant 40.31 can be calculated from the physical quantities electron mass, electron charge, and the permittivity of free space.

In the case to DF observations the dispersive nature of the ionosphere can be exploited by building the IF-LC introduced in Section 2.3.3. It allows for the complete elimination of (first order) ionospheric delay and much better accuracy of the gained parameters. For positioning with SF receivers however, using Equations 4.16 is of great importance in terms of accuracy. They allow the user to correct for the path delay using TEC values and the well-known carrier frequencies. TEC information can be obtained from various sources including:

\section{Global VTEC models}

\section{Regional VTEC models}

\section{Ionospheric prediction models}

\section{Empirical models}

\subsubsection{Tropospheric delay}

In contrast to the ionosphere, the troposphere is a non-dispersive medium for electromagnetic waves up to $15 \mathrm{GHz}$. No larger number of charged particles is present in the layer and therefore all carrier frequencies are delayed by the same amount. Although the magnitude of the delay is typically much smaller, the non-dispersive nature does not allow elimination by building linear combinations.

The tropospheric delay is usually split up into two parts (Davis et al. 1985): 
- Hydrostatic part: mainly determined by atmospheric pressure

- Wet part: delay due to amount of water vapour

Furthermore one can distinguish between delays in different directions. This is typically done for the zenith direction (zenith delay) and elevation direction of each satellite (slant delay). In the following these kind of delays are introduced in detail and some recipes for their modelling and estimation are given.

\section{Zenith delay}

The Zenith Total Delay (ZTD) represents the full delay in zenith direction, due to the presence of the troposphere. The definition of the ZTD reads (Jin and Luo 2009):

$$
\Delta \rho^{z}=c \cdot \tau=10^{-6} \cdot \int_{0}^{\infty} N(s) \cdot d s
$$

where $c$ is the speed of light in a vacuum, $\tau$ is the delay measured in seconds, and $N$ is the neutral atmospheric refractivity, where neutral addresses the non-ionized component of the atmosphere.

Again following e.g. Jin and Luo (2009) $N$ can also be computed by:

$$
N=k_{1} \cdot \rho+k_{2} \cdot \frac{P_{w}}{Z_{w} \cdot T}+k_{3} \cdot \frac{P_{w}}{Z_{w} \cdot T^{2}}
$$

where $k_{i}(i=1,2,3)$ are constants, $\rho$ the total mass density of the atmosphere, $P_{w}$ the partial pressure of water vapor, $Z_{w}$ a compressibility factor close to unity accounting for the small departures of moist air from an ideal gas, and $T$ the temperature in Kelvin.

The term can be split up into a hydrostatic (dry) and a wet proportion, $N_{h}$ (addressing the first term of 4.19) and $N_{w}$ (addressing the other two terms of 4.19).

As indicated in Figure 4.4, these two quantities differ substantially in both magnitude and variability. The dry part is far greater in magnitude and shows a continuous decay with height, whereas the wet part is of smaller magnitude but very variable in both time and space. Its fluctuations depend heavily on atmospheric conditions (water vapour pressure, temperature) as Equation 4.19 and Figure 4.4 indicate.

Two effects have to be considered, when evaluating the integral in Equation 4.18. At first, propagation speed is decreasing with increasing density, which represents the main influence on the signal. Secondly, the signal propagation is determined by Fermat's principle, stating that the signal path is not necessarily a straight $(G)$ but bent line $(S)$ as indicated in Figure 4.5. This effect can be accounted for by extending Equation 4.18 to

$$
\Delta \rho^{z}=c \cdot \tau=10^{-6} \cdot \int_{0}^{\infty} N(s) \cdot d s+S-G
$$



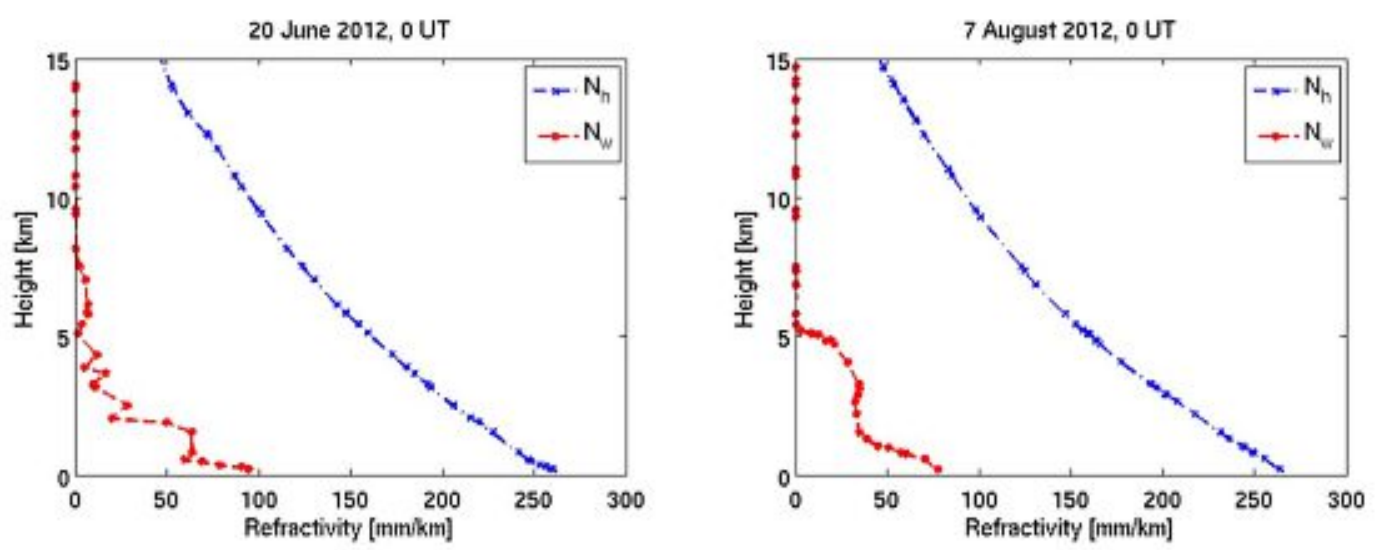

Figure 4.4: Height profiles of the hydrostatic $\left(N_{h}\right)$ and wet component $\left(N_{w}\right)$ of the refractivity $N$ for two different days, originating from the same time of the day and station. $N_{h}$ shows a continuous curve, $N_{w}$ high variability in time and space. Taken from Nilsson et al. (2013).

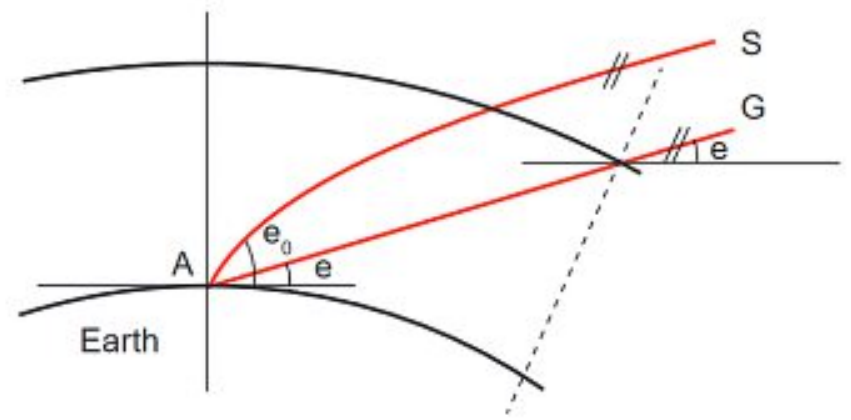

Figure 4.5: Path of a GNSS signal trough the troposphere. The actual path $\mathrm{S}$ is longer than the geometric path $\mathrm{G}$ due to the bending effect and arrives at a different elevation $e_{0}$. Taken from Nilsson et al. (2013).

In practical applications the effect is only relevant for observations gathered at very low elevations. The magnitude of the full tropospheric path delay is typically in the metre-range, although reaching up to ten times higher values at very low elevations.

As mentioned before, the ZTD can, just as the refractivity, be split up into two parts, the Zenith Hydrostatic Delay (ZHD) and the Zenith Wet Delay (ZWD).

The ZHD is defined as the integral over the hydrostatic refractivity along the signal path:

$$
\Delta \rho_{h}^{z}=\int_{0}^{\infty} N_{h}(s) \cdot d s
$$

and can be modelled by only using surface pressure, station height and latitude, following simple models like Saastamoinen (1972), as shown in Equation 4.22:

$$
\Delta \rho_{h}^{z}=10^{-6} \cdot \frac{K_{1} \cdot R_{d} \cdot p_{s}}{g_{m} \cdot\left(1-0.00266 \cdot \cos (2 \cdot \Phi)-0.28 \cdot 10^{-6} \cdot H\right)}
$$


where $K_{1}$ is the refractivity constant of dry air, $R_{d}$ is the specific gas constant of dry air, and $p_{s}$ is the surface pressure. $p_{s}$ is corrected for local gravity effects by using mean gravity $g_{m}$, station latitude $\Phi$ and the station height above the geoid $H$.

ZWD can then be derived as the simple difference between ZTD and ZHD as

$$
\Delta \rho_{w}^{z}=\Delta \rho^{z}-\Delta \rho_{h}^{z}
$$

It represents the tropospheric delay of the GNSS signal caused by water vapour and can be transformed into precipitable water vapor (PWV) by

$$
P W V=\Pi \cdot \Delta \rho_{w}^{z},
$$

where the conversion factor $\Pi$ equates to

$$
\Pi=\left(10^{-6} \cdot \rho \cdot R_{v} \cdot\left(\frac{k_{3}}{T_{m}}+k_{2}^{\prime}\right)\right)^{-1},
$$

with $\rho$ being the density of liquid water at $0^{\circ} \mathrm{C}, R_{v}$ the specific gas constant for water vapour, $T_{m}$ the weighted mean temperature of the atmosphere, $k_{2}^{\prime}=k_{2}-m k_{1}$ and $m$ the ratio of the molar masses of water vapour and dry air.

\section{Slant delay}

The tropospheric delay in line-of-sight direction is called Slant Total Delay (STD). It is obtained by a scaling of the zenith component with geometric factors, the so-called mapping functions which are again split up into hydrostatic and wet part. As a first approximation, with evenly stratified atmosphere and no earth curvature assumed, one can write (Karabatic 2011) the STD as

$$
\Delta \rho^{s}=\Delta \rho^{z} \frac{1}{\sin \epsilon}
$$

where $\Delta \rho^{s}$ is the tropospheric slant delay, $\Delta \rho^{z}$ is the tropospheric zenith delay and $\epsilon$ is the elevation angle of the satellite.

The factor $1 / \sin (\epsilon)$ denotes a crude basic version of a mapping function which does not account for azimuthal asymmetry. Simple approaches like this one deliver reasonable STD results only for elevations above $10^{\circ}$. For lower elevation angles a number of more sophisticated mapping functions were developed over the course of the last 40 years. The next section aims to give a short overview over them.

\section{Mapping functions}

Mapping functions condense information about the variability of delays over the whole elevation range (Landskron and Böhm 2017). This information is typically expressed by 
the three mapping function coefficients $a, b$ and $c$ of a continued fraction form (Marini 1972)

$$
m f(\epsilon)=\frac{1}{\sin \epsilon+\frac{a}{\sin \epsilon+\frac{b}{\sin \epsilon+\frac{c}{\sin \epsilon+\ldots}}}}
$$

These coefficients, independent of the azimuth angle, are determined for the hydrostatic and the wet part separately. This can be done by fitting them to different sources of observations/model data:

- Standard atmospheres: e.g. Chao (1974)

- Radiosonde data: Niell (1996)

- NWP data: Niell (2000) and Böhm et al. (2006).

Marini (1972) conducted the first tryouts of verification against a standard atmosphere. Here, he found an agreement between them better than $0.3 \%$ down to elevations of $1^{\circ}$. However, a comparison with radiosonde data by Niell (1996) showed large deviations of range corrections in both low elevations and even in zenith direction.

Herring (1992) introduced a slightly different fraction form compared to Marini (1972), shown in Equation 4.28.

$$
m(\epsilon)=\frac{1+\frac{a}{1+\frac{b}{1+c}}}{\sin \epsilon+\frac{a}{\sin \epsilon+\frac{b}{\sin \epsilon+c}}}
$$

In 1996, Niell (1996) developed the Niell mapping function (NMF) which is based on the continuous fraction form shown in Equation 4.28. The coefficients $a$ and $b$ are only dependent on day of year (DOY) and the station latitude and height. For the Northern Hemisphere they are derived from meteorological profiles of the US Standard Atmosphere (see Chapter 3, Figure 3.2).

Since the millennium a number of NWP-based mapping functions have been developed: In 2000, Niell (2000) introduced the Isobaric Mapping Function (IMF), which are based on the relation of hydrostatic mapping function coefficients with geopotential height at isobaric surfaces. The coefficients are derived from 6-h model output of a global NWM. Based on this concept the Vienna Mapping Functions (VMF) were introduced by Böhm and Schuh (2004), which were able to overcome some limitations of IMF, especially in the wet part.

The subsequent Vienna Mapping Functions 1 (VMF1) by Böhm et al. (2006) are regarded as the most accurate mapping functions nowadays and are applied by numerous research centers and other agencies worldwide (Landskron and Böhm 2017). Analogously for North America, the UNB-VMF1 (Santos et al. 2012) use the same model but NWM 
data from the National Centers for Environmental Prediction (NCEP) and the Canadian Meteorological Center.

The latest version of the VMF series is VMF3 (Landskron and Böhm 2017), which introduced some refinements, especially for very low elevation angles. 


\section{Chapter 5}

\section{GNSS Meteorology}

This chapter aims to give a short overview on the history of GNSS Meteorology and the different approaches used nowadays. Especially in Europe a number of dense station networks, initially built up for positioning services, were utilized for tropospheric monitoring over the last decade. Some of these networks are also described in the following chapter.

Furthermore an introduction to the means of using this data in weather forecasting shall be given. In this context different strategies for assimilation of GNSS products into numerical weather models (NWM) are outlined.

Most information is based on Guerova et al. (2016), who give a very detailed insight into the current state and future prospects of GNSS Meteorology.

\subsection{History}

The term GNSS Meteorology was first used by Bevis et al. (1992), who presented the basic concept of the technique in the early nineties. GNSS Meteorology uses the tropospheric delays introduced in Section 4.2.2 to derive estimates of water vapour content, which can be used in NWP data assimilation. Figure 5.1 illustrates the basic principle of the approach here once again.

Since the rise of GNSS as an accurate positioning and navigation system, many station networks have been established worldwide. Data from these networks are often free of charge for scientific research and therefore also exploited to derive information on atmospheric conditions. The first continuous GPS network built up for meteorological purposes alone was the GPS-IPW Network of the National Oceanic and Atmospheric Administration (NOAA) led by their Forecast System Laboratory in the USA in the 1990s (Wolfe and Gutman 2000). In the course of the last decade, the technique has 


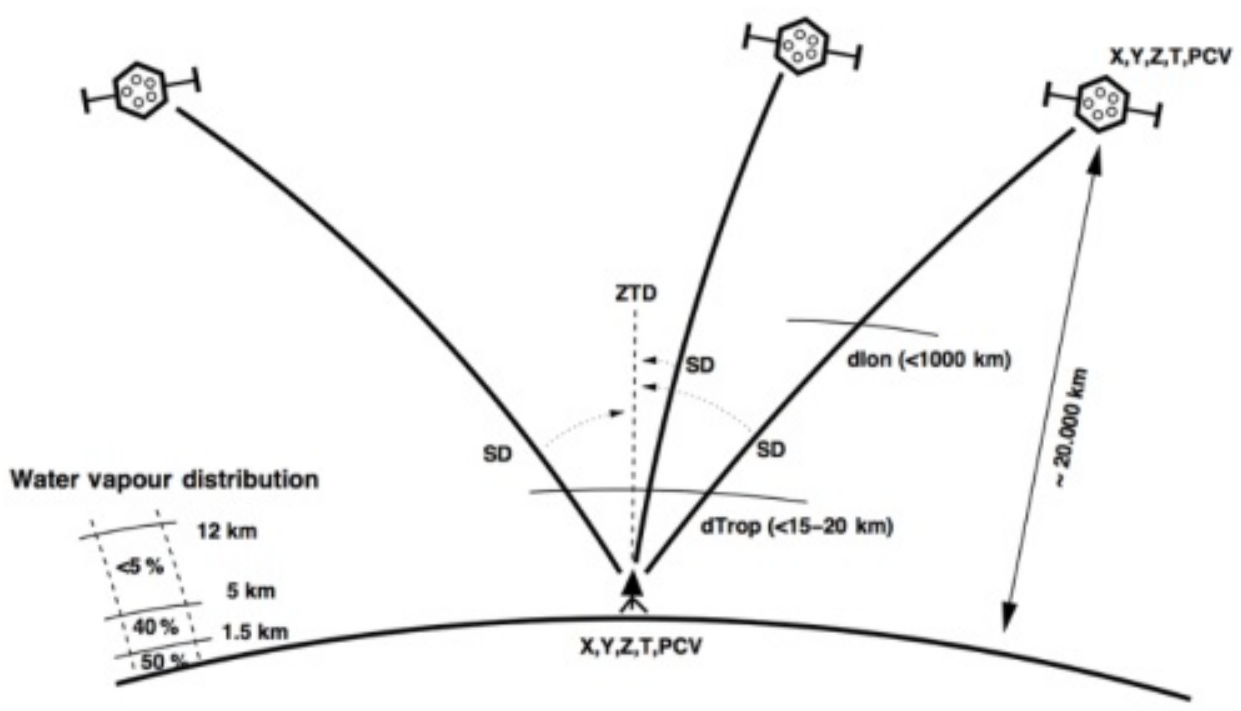

Figure 5.1: Basic illustration of the GNSS Meteorology concept. Individual STDs are mapped to ZTD for a single station. Taken from Guerova et al. (2016).

become more feasible and more and more observations are available for assimilation. In Europe, this fact relies heavily on the set-up of different research programs to investigate the usability of GNSS-derived information in meteorology. The first one of those was founded by The European Cooperation in Science and Technology (COST) and was called "COST Action 716: Exploitation of ground-based GPS for climate and numerical weather prediction applications" (Elgered 2001). Following up, the project "Targeting Optimal Use of GPS Humidity measurements in meteorology (TOUGH)" was conducted from 2003 to 2006 (Huang et al. 2003). Based on the success of the prior research, the EUMETNET EIG GNSS water vapour programme (E-GVAP) was established in 2005. It consists of a series of research projects all devoted to the use of near real-time (NRT) tropospheric GNSS data for NWP. A number of analysis centers from all over Europe contribute to E-GVAP by providing delay estimates for forecast validation, NRT monitoring and research projects.

Figure 5.2 gives an overview on the timeline of European projects mentioned above. In addition to research projects embedded in the projects mentioned above, several other research studies have been carried out.

\subsection{GNSS-based troposphere products for NWP}

As already outlined in the previous section, tropospheric products from GNSS have attracted increasing interest of the meteorological community over the last decade. This 


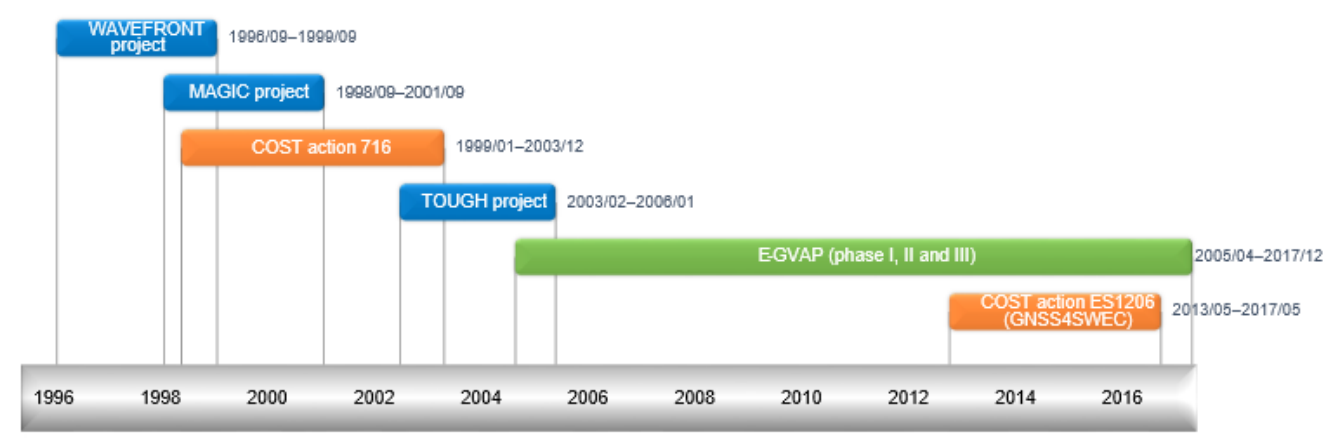

Figure 5.2: Timeline of European projects in the field of GNSS Meteorology from 1996 2016. Taken from Guerova et al. (2016)

went from small case studies with only few GNSS observations available for assimilation, to operational usage of (at least ZTD) products in most NWP models today. Nowadays they are a well-established and valuable observation source for data assimilation in NWP models. The field of GNSS meteorology therefore denotes a strong interaction and cooperation between GNSS experts and meteorologists, aiming to increase availability and quality of the observations as well as proper usage in applications.

Due to the variety of products it is reasonable to give a short overview on the most popular ones used in NWP in this section. In a very basic manner, products can be grouped in ground-based (GNSS meteorology) and space-based (GNSS Radio Occultation). Although a short introduction on GNSS Radio Occultation (GNSS-RO) is provided, this section focuses mainly on ground-based products.

\subsubsection{Ground-based products}

\section{Zenith Total Delay/Integrated Water Vapour}

Up to date, ZTD is still the most common tropospheric product from GNSS processing. The majority of NWP nowadays is able to assimilate ZTD observations or quantities derived from it, like Integrated Water Vapour (IWV). Table 5.1 gives an overview of popular NWP models which use ZTD/IWV. Nevertheless other techniques and quantities, such as GNSS Tomography and usage of STDs, are exploited quite frequently nowadays. Especially STD currently denotes a scientific focus of the community since the slant estimates contain a larger amount of information as the rays also experience horizontal variations of atmospheric constituents. 


\begin{tabular}{lcc} 
Model & Quantity & Assimilation strategy \\
\hline WRF & ZTD/STD & 4D-Var \\
COSMO & IWV/STD & 4D-Var \\
AROME & ZTD/STD & 3D-Var \\
ECMWF (IFS) & ZTD & 4D-Var
\end{tabular}

Table 5.1: Overview of assimilation strategies used for GNSS-derived tropospheric products in popular NWP models: WRF (Weather Research and Forecasting), COSMO (Consortium for Small-scale Modeling), AROME (Application of Research to Operations at MEsoscale) and IFS (Integrated Forecasting System, operated at ECMWF) model

\section{GNSS Tomography}

GNSS Tomography makes use of existing GNSS infrastructure (satellites and networks of geodetic reference stations) in order to derive a spatially resolved water vapour field. Therefore, a large number of slant delays are estimated from a network of ground stations using double-difference network processing (Möller 2017) or PPP (Zhao et al. 2018). Since every satellite-station combination gives an integral measure for water vapour concentration along the ray path, a dense observation field can be created given a sufficient number of ground stations (see Figure 5.3). This tomography area is then usually divided into 3D cubes called voxels. Then a tomographic reconstruction (as commonly known from seismology) is carried out using these slant delay observations to derive a 3D field of wet refractivity and ultimately atmospheric water vapour. The main challenge of the approach is the ill-posed nature of the mathematical problem behind the technique. This originates from a limited spatial resolution of satellites and ground stations and therefore, especially for a high resolution field, not every voxel will be intersected by a signal ray. Thus, from mathematical point of view, a singular design matrix has to be inverted to solve the equation system. Therefore, special algorithms for under-determined systems have to be applied. Recent investigations deal for instance with different parametrization methods for computing the design matrix. Adavi et al. (2020) studied the effect of introducing ray-tracing methods (see e.g. Hofmeister (2016)) for computing the length of rays passing through a voxel as well as the impact of considering the topography of the tomography model area. They found improvements of about $5 \mathrm{ppm}$ in mean deviation of wet refractivity to radiosonde measurements.

Assimilation of tomography products into NWMs was also shown to be beneficial by a number of studies, e.g. Hanna et al. (2019) who used a RO assimilation operator to assimilate refractivity fields. 
More detailed insights on the principles, benefits and restrictions of the technique of the are given e.g. by Möller (2017).

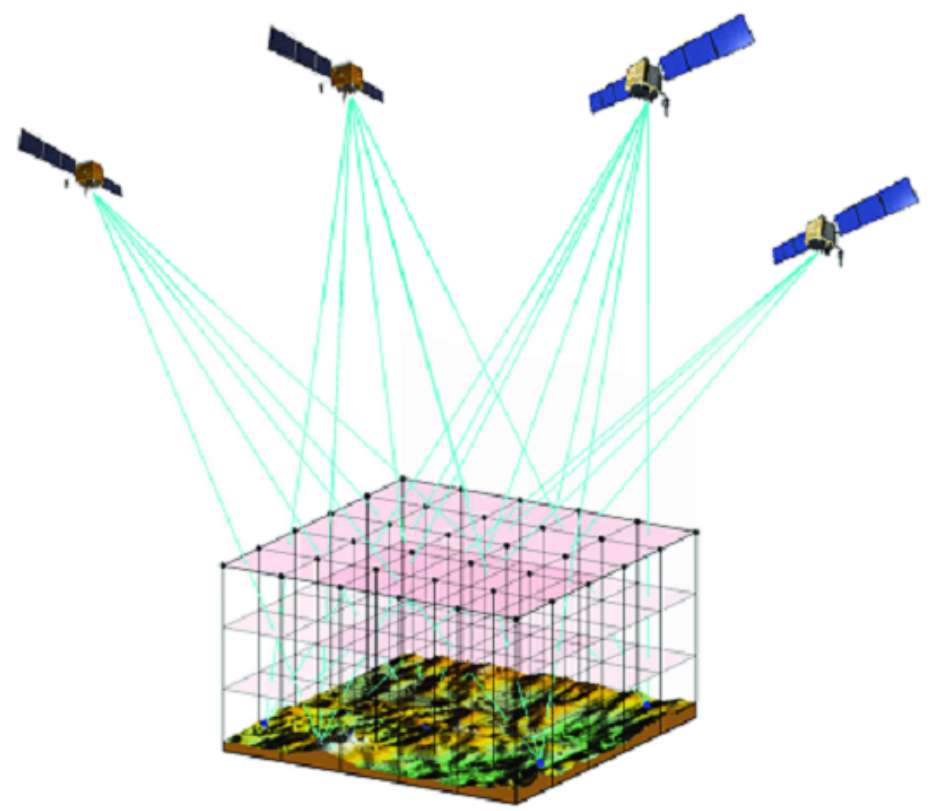

Figure 5.3: Principle of GNSS tomography, taken from Dong and Jin (2018)

\subsubsection{Space-based products}

GNSS Radio Occultation (GNSS-RO) denotes the most prominent space-based technique for estimation of meteorological information about the lower (troposphere) as well as the higher atmosphere (ionosphere). The technique is based on using GNSS measurements received by low-Earth orbiting (LEO) satellites to profile the Earth's atmosphere with high vertical resolution and global coverage. Beginnings of radio occultation techniques go back to the 1960s where they were used to study atmospheres of the outer planets of the solar system. Over the last decades it was increasingly used to probe the Earth's atmosphere also.

As radio signals from the GNSS satellites pass through the atmosphere, molecules and electrons bend their paths and slow their propagation. The benefits as well as disadvantages of GNSS-RO are listed in the following:

- Assured long-term stability $(+)$

- All-weather operation $(+)$

- Global 3D coverage $(+)$

- Vertical resolution: $100 \mathrm{~m}$ in the lower troposphere $(+)$ 
- Compact, low-power, low-cost sensor $(+)$

- High accuracy: Averaged profiles to $<0.1 \mathrm{~K}(+)$

- Fairly low number of soundings per LEO (depending on GNSS systems used): 600 per day (-)

- Poor spatial resolution (300 km horizontal) (-)

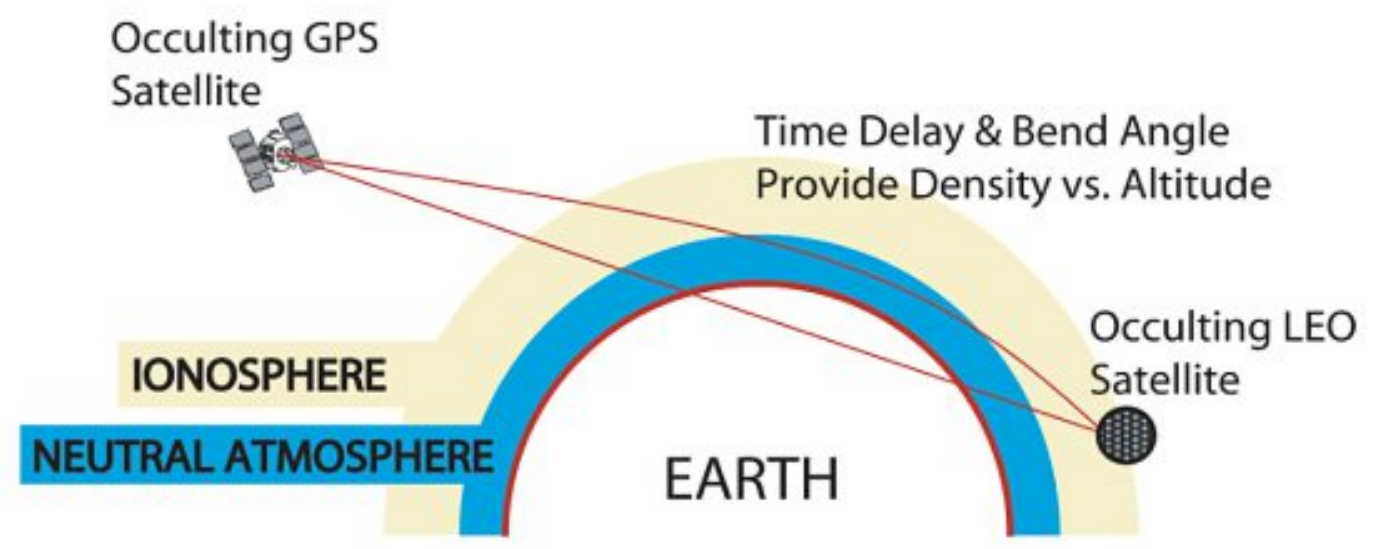

Figure 5.4: Basic principle of GNSS RO, Source: ECMWF

Nowadays GNSS RO is extensively used in for NWP and climate research (Anthes 2011). Different scientific programs like the COSMIC mission have been built up in order to establish global data coverage. Furthermore many low-orbiting satellites originally launched for gravity field observation like CHAMP or GRACE have been used for RO (see e.g. Wickert et al. (2009)).

\subsection{Estimation of tropospheric parameters using PPP}

Tropospheric parameters are typically derived together with station coordinates and the receiver clock error in GNSS analysis. The mathematical means to carry out this task will be introduced in Chapter 6, Section 6.3 in from of standard methods for parameter estimation. Nevertheless, it should already be noted here that estimated tropospheric parameters are typically strongly correlated with the other parameters estimated, especially to the height coordinate and the receiver clock error. Time series of height and ZTD typically show a high anti-correlation and therefore their respective estimation errors are also dependent on each other. If ZTDs are estimated in GNSS analysis, the respective stations height coordinates are decreased by a magnitude of 
3-3.5 × ZTD (Beutler et al. 1989). As shown in Beutler et al. (1989) or Krawinkel et al. (2014), the transfer ratio between height coordinate and ZTD residual is constant and corresponds to approximately $-3: 1$.

The exact ratio between height and ZTD error is dependent on the elevation cut-off used in processing. The lower the cut-off elevation angle used in processing, the more efficiently ZTD can be decorrelated from the height coordinate. Nevertheless, observations from such low elevations are often not available or significantly affected by multipath and therefore have to be used with caution, especially in kinematic scenarios.

Over the last years PPP is becoming an increasingly used technique for tropospheric parameter estimation. Although DD network solutions are still considered in a lot of operational processing at different data centers, a number of authors have already shown promising results for PPP processing approaches. The analysis centers at GFZ and NGAA (GNSS AC collaboration between Chalmers Technical University and Swedish Meteorological and Hydrological Institute) developed their own techniques for providing global orbit and clock products in support of PPP (Guerova et al. 2016). Tropospheric products derived from real-time PPP processing are of high interest for NWP models and for nowcasting of severe weather events (Guerova et al. 2016).

Simeonov et al. (2016) evaluated IWV from WRF with PPP results over Bulgaria and found good agreement between the data sets. Stepniak and Bock (2018) compared one year of GPS PPP and DD results in terms of ZTD and found that PPP processing might be a beneficial approach compared to DD, especially in the presence of outliers arising from defects in the baseline geometry.

In Austria, first studies were conducted by Karabatic (2011), who found slightly worse results for ZWD from PPP compared to those from DD processing for a subset of stations of the EPOSA network. Nevertheless the possible benefits of PPP processing like shorter latency time could already be outlined by the study. Since then several research projects on similar topics were conducted at the research division Higher Geodesy at TU Wien which indicated that shorter latency is often equally important as highest precision, for typical NWP applications. 


\subsubsection{Kinematic platforms}

This section is specifically devoted to GNSS Meteorology (i.e. ZTD estimation) in kinematic GNSS scenarios like when utilizing air- or shipborne receiver data. Kinematic GNSS must deal with the dynamics of a receiver, and the problem of being unable to constrain the position solution to a single location. This reduces the redundancy of the system and therefore tends to worsen the accuracy of the tropospheric estimates.

Only very few studies have been conducted in this direction of research up till now. Rocken et al. (2005) used DD relative solutions with levered ZTD estimates, where ZTD at a static reference site is fixed and only the difference of a kinematic rover to this reference is estimated. Shipborne studies were carried out by Dodson et al. (2001) and Kealy et al. (2012), using a levered approach to obtain an agreement of 1-2 mm in ZTD and $2.2 \mathrm{~mm}$ in PWV. Chadwell and Bock (2001) processed a network-equivalent double-differenced ambiguity-fixed solution for a GPS buoy and obtained an agreement of $1.5 \mathrm{~mm}$ PWV with radiosonde launches few kilometres away.

An extension of these studies to the railway domain and therefore to highly kinematic conditions (up to $200 \mathrm{~km} / \mathrm{h}$ travelling speed) is carried out in this thesis, using specific processing (Chapter 6 ), validation and assimilation methods (Chapter 7 ). 


\section{Chapter 6}

\section{GNSS data processing}

The following section gives a detailed overview of all processing steps carried out on the raw GNSS tracking-data. Therefore the chapter covers an introduction on the utilized datasets, continues with extensive pre-processing procedures, the actual PPP algorithm applied and finally discusses suitable post-processing strategies. For certain tasks (e.g. pre-processing) multiple approaches were tested and a quantitative evaluation of their weaknesses and strengths is provided.

\subsection{Data sources}

This section introduces the data utilized in GNSS processing within this study. This ranges from the actual GNSS measurements taken on trains to the reference station data and track database used for specific processing strategies.

\subsubsection{Greenlight}

Greenlight is a project by the Austrian Federal Railways (ÖBB) which deals with accurate train positioning to support a number of applications from improved passenger information to security activities. The basic idea is to build and operate an integration platform (Greenlight gateway) for different ÖBB systems established in-house. This provides the chance of developing new functions for train positioning as well as for the in-house passenger information system (AURIS). Figure 6.1 gives an overview of the workflow and over all systems working together on the Greenlight gateway. The basic workflow can be summarized as the following:

1. Observations from GNSS are recorded by an antenna installed on the top of train traction vehicles. 
2. From the central reference station database (TEPOS) correction data are broadcasted to the receiver.

3. RTK-Positions are calculated at the receiver and position data are broadcasted back to TEPOS via GSM.

4. Position data are forwarded from TEPOS to the ARAMIS (Advanced Railway Automation Management Information System) and AURIS (Automatisches Reisenden-Informationssystem) systems.

5. In the Greenlight gateway the position data is linked to data from the route systems and the rail database. This is complemented by additional data for locations with low or no reception.

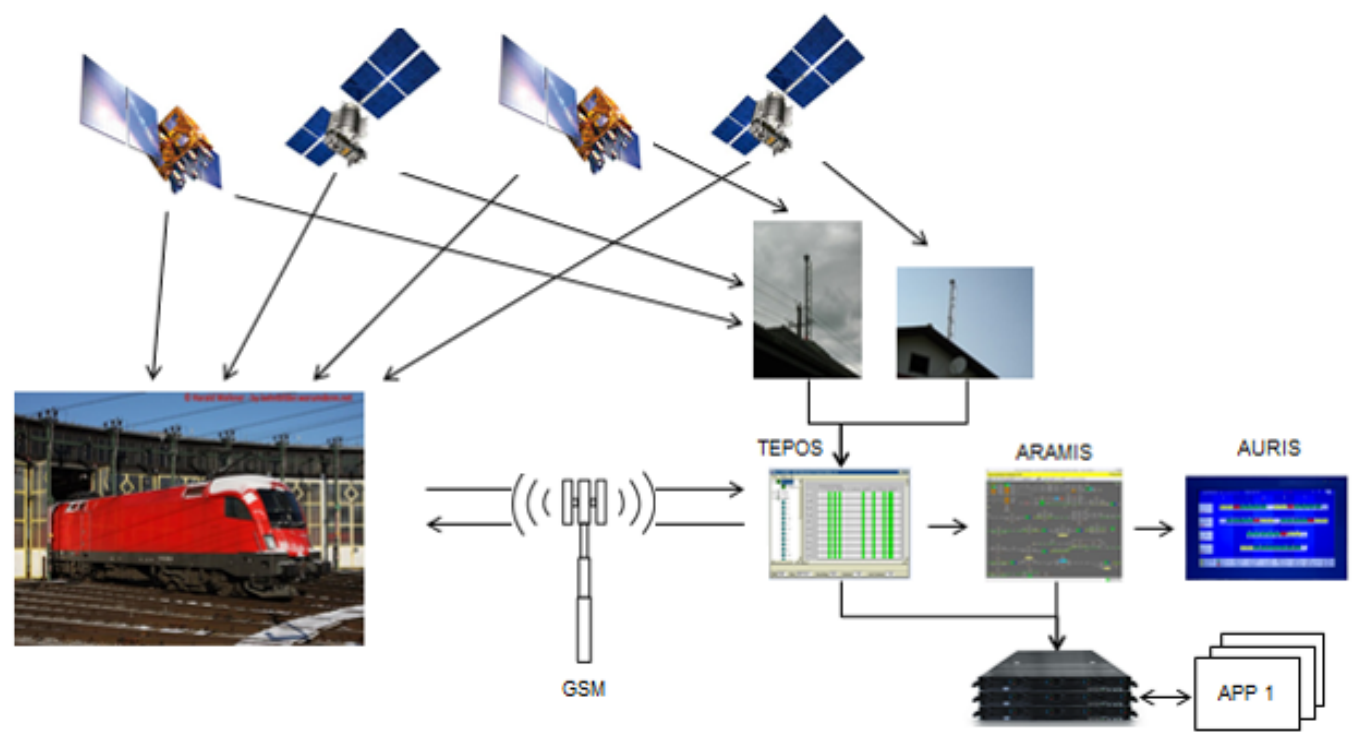

Figure 6.1: Overview of systems working together in Greenlight, courtesty of DI (FH) Manfred Stättner, ÖBB Infrastrucktur

In 2016 in the course of the Greenlight project, ÖBB started to equip their fleet of trains with multi-GNSS receivers with the aim of online-positioning. The receivers are capable of logging GPS and GLONASS L1 observations at an update rate of $1 \mathrm{~Hz}$. The upcoming generation of receivers will be GPS and Galileo L1 capable and in future also DF capability is contemplated. Today, more than 1400 vehicles are already equipped with such single-frequency receivers, providing decimetre localisation of the fast-moving traction vehicles gained by RTK-positioning and referencing to the EPOSA correction service. In parallel, the receivers also allow to store raw GNSS observation data of all 
train travels within a period of up to three weeks.

These data, acquired for a number of days, form the foundation of all results shown in the next chapters.

\subsubsection{Railway track database}

Furthermore, ÖBB generously provided a coordinate database for two of the investigated railway tracks (Salzburg - Klagenfurt/ Innsbruck - Graz). In the first place, this data are used for quality checks of the PPP solutions in terms of positioning performance. Secondly, the height information from the database is utilized for a height-constrained PPP approach (see Section 6.8 for detailed information).

\subsubsection{EPOSA}

Data from the Austrian GNSS reference station network EPOSA is utilized for correcting the train-tracking GNSS data for ionospheric influence (using the SEID algorithm, see Section 6.4). Therefore a short introduction to the network and its characteristics is given in the following.

The GNSS network consists of 38 stations, which are distributed all over Austria

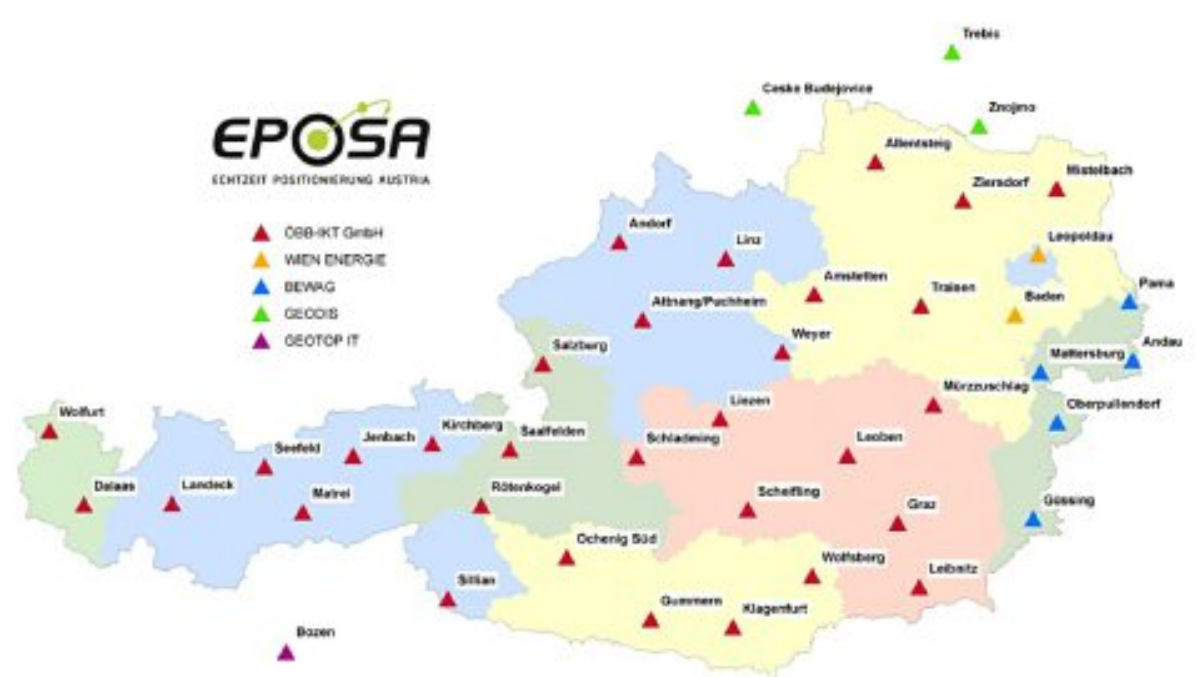

Figure 6.2: Reference station network of EPOSA. The colour of the triangles denotes the company operating the station. Taken from http://www.eposa.at/

(Figure 6.2). EPOSA originated from a cooperation of different Austrian companies and started operating in autumn of 2009. Main goal is to provide GNSS users with realtime correction data, by using all available GNSS systems, at highest possible reliability and guaranteed quality. The reference station network (Figure 6.2) was built up in 
consideration of several criteria like present infrastructure, stability of reference points, distribution of stations and distances in between and obstruction free horizon. Every reference station is equipped with both a GPS and a GLONASS receiver (in a future state also Galileo). The research division Higher Geodesy at the TU Vienna provides quality control for all station data on a regular basis. Furthermore the network is utilized for estimation of tropospheric parameters which are delivered to the Austrian Central Institute of Meteorology and Geodynamics (ZAMG) for assimilation into the NWP model AROME.

\subsection{Pre-Processing}

Due to the highly kinematic and multipath-prone nature of the utilized data, a data pre-processing is necessary before starting the actual estimation. Therefore, a cycle slip correction is applied in order to correct phase measurements. Since different methods exist for this task, a selection of 2-3 strategies will be investigated. Furthermore, the ionosphere has to be accounted for since the observations are only single-frequency. Here two different approaches will be implemented. The first one uses existing ionospheric TEC models to calculate range corrections for the observations. Different models are available at both global and regional scale. At the research division Higher Geodesy, the global model GIOMO (Magnet 2019) and the regional model Regiomontan (Boisits et al. 2020) are available. These models were not used in this study due to partly expensive implementation in the utilized software packages but might be in further studies on this topic.

In the second approach, the utilized SF data will be extended to DF observations using the SEID algorithm in order to apply the ionospheric correction by building a IF-LC in the PPP processing later on.

\subsubsection{Dilution of Precision}

The geometry of the satellites (i.e. how the user sees them) affects the positioning error (Sanz Subirana et al. 2013). This influence can be quantified by the so-called Dilution Of Precision (DOP) parameters. They are defined as (e.g. Sanz Subirana et al. (2013)):

- Geometric Dilution Of Precision:

$$
G D O P=\sqrt{q_{x x}+q_{y y}+q_{z z}+q_{t t}}
$$


- Position Dilution Of Precision:

$$
P D O P=\sqrt{q_{x x}+q_{y y}+q_{z z}}
$$

- Horizontal Dilution Of Precision:

$$
H D O P=\sqrt{q_{n n}+q_{e e}}
$$

- Vertical Dilution Of Precision:

$$
V D O P=\sqrt{q_{u u}}
$$

- Time Dilution Of Precision:

$$
T D O P=\sqrt{q_{t t}}
$$

where $q_{x x}, q_{y y}, q_{z z}$ and $q_{t t}$ represent the diagonal entries of the cofactor matrix (see Section 6.3 for detailed description) related to the 3D-coordinates in a geocentric earthfixed frame (subscripts $x x, y y$ and $z z$ ) as well as the receiver clock error (subscript $t t$ ). On the other hand, HDOP and VDOP are defined in a local level frame and therefore $q_{n n}, q_{e e}$ and $q_{u u}$ refer to the diagonal entries of the cofactor matrix related to the 3Dcoordinates in this respective frame.

GDOP is presented together with the number of visible satellites in the first analyses of the raw data in Chapter 8. Both parameters help to quantify the environmental conditions along the investigated tracks in terms of satellite geometry. Therefore they provide a first indication of the resulting quality to be expected from PPP processing and help top identify possibly critical sections along the routes where results might be degraded or unreliable.

\subsubsection{Multipath analysis}

Train-borne GNSS receiver observations are typically multipath afflicted due to the permanently changing and often challenging environment. Therefore a multipath analysis was carried out, in order to get an idea of the quality of the raw observations and identify particularly critical sections on the investigated tracks. The procedure chosen in the course of this study uses the pseudo-multipath or Code-Minus-Carrier (CMC) observable:

$$
C M C^{s}=P^{s}-L^{s}
$$

where $P^{s}$ and $L^{s}$ denote code/pseudorange and carrier phase measurements (in meter) from each satellite. It represents a judicious linear combination of the GPS observables 


\begin{tabular}{lccc} 
Method & Accuracy & Computational cost & Additional requirements \\
\hline Phase-code & affected by code noise & low & no \\
Phase-phase & wavelength dependent & medium & DF observations \\
Doppler Integration & affected by high dynamics & low & no \\
Inertial aided & high & medium & INS system (costs, quality, ...) \\
Time-differencing & proportional to order of time series & high & DF observations in some cases \\
Double-differencing & varies with algorithm complexity & high & no
\end{tabular}

Table 6.1: Overview on methods for cycle slip detection (Farooq et al. 2018).

and produces an observable containing a biased estimate of the pseudorange multipath and a few small additional terms (Bisnath and Langley 2001).

The pseudo-multipath observable gives a good representation of code multipath, as the magnitude of the carrier-phase multipath in Equation 6.6 is much smaller than the corresponding pseudorange terms.

\subsubsection{Cycle slip detection}

A cycle slip denotes a jump in the accumulated carrier phase observation by an integer number of cycles. Its occurrence affects not only the current measurement, but also the following epochs and therefore can substantially degrade positioning accuracy.

\section{SF observations}

Although generally considered difficult, cycle slip detection and correction for single frequency receivers has recently received attention due to the large number of evolving applications for low-cost mass-market single frequency receivers. According to HofmannWellenhof et al. (2012), three sources of cycle slips can be identified:

1. Physical obstructions, blocking the signal,

2. Low signal to noise ratio,

3. Failure in the receiver software.

A variety of techniques for cycle slip detection and correction exist for both DF and SF observations. Farooq et al. (2018) give a good overview on the current status of methods for SF data and the categorisation in Table 6.1 is based on their explanations. In this study, Doppler Integration and the Time-differencing approach were tested for pre-processing of raw tracking data.

Doppler-Integration was found unsuitable for the tested cases since the receiver dynamics were too high at typical travelling speeds (mean around $100 \mathrm{~km} / \mathrm{h}$ ). 
Although computationally quite expensive (depending on the order of differencing), timedifference-based approaches were found to be most useful for the investigated SF data.

\section{DF observations}

For DF data like from the EPOSA stations or generated with the SEID algorithm, phasephase combinations like the MW-LC (see Section 2.3.3) were utilized. The GF-LC could also be used for identifying cycle slips for reference station data. Based on computed values of these LCs, abnormally large values in epoch-differences are identified as cycle slips and corrected or excluded from processing (depending on the software implementation). Both methods are e.g. realized in different software packages which were used for processing.

\subsubsection{Data cleaning}

Since the train data is prone to multiple error sources outlined above, a quality check has to be carried out and faulty data has to be rejected in the best possible way.

This quality check must also be applied to the DF reference station data. An important prerequisite for applying SEID successfully to a SF station is to have the most consistent and clean reference station data possible. Therefore a pre-processing of this data was carried out in order to detect outliers and cycle slips, which denote a major error source for SEID.

This pre-processing is done using goGPS (Herrera Olmo et al. (2015) and Realini and Reguzzoni (2013)), an open-source satellite positioning software package written in MATLAB. The methods described above were utilized for this step.

\subsection{Parameter estimation in PPP processing}

From the equations presented in Chapter 2, Section 2.3 the wanted set of parameters (typically 3D coordinates, receiver clock error and ZTD) can be derived. Since typically redundant observations are available, an appropriate technique for an optimal estimation of the parameter set has to be applied.

Beside optimal results of the unknown parameters, another huge benefit of such techniques is that additionally error estimates for these parameters can be calculated by introducing a stochastic model in the estimation. The error estimates are typically described by the covariance (or variance-covariance) matrix of the respective parameters. Therefore the calculation of the covariance matrix of the wanted parameters is also in- 
troduced for the respective approaches presented in the following.

The two (most common) techniques for parameter estimation in GNSS processing are introduced in the following:

- Least Squares (LSQ) adjustment

- Kalman Filtering

\subsubsection{Least-squares adjustment}

The most common technique for parameter estimation in GNSS positioning is the classical LSQ adjustment, which is still utilized in a number of GNSS software packages nowadays. In this study, LSQ adjustment is used for parameter estimation within the CSRS-PPP (see Section 6.5) software and therefore the basic principle is introduced in the following.

Using the notation of Hofmann-Wellenhof et al. (2012) and Magnet (2019), the basic functional relationship of LSQ reads:

$$
\boldsymbol{L}+\boldsymbol{v}=\phi(\boldsymbol{x})
$$

where $\boldsymbol{L}([n \times 1])$ denote the measured observations (with $n$ being the number of observations), $v$ the error vector and $\phi(\boldsymbol{x})$ the functional relation between the observations and the parameters to be estimated. $\boldsymbol{x}=\boldsymbol{x}_{0}+\boldsymbol{d} \boldsymbol{x}$ denotes the vector of unknown parameters with $\boldsymbol{x}_{0}$ being approximate values and $\boldsymbol{d} \boldsymbol{x}$ the difference to their estimated values.

Linearisation of Equation 6.7 gives

$$
\boldsymbol{L}+\boldsymbol{v}=\phi\left(\boldsymbol{x}_{0}\right)+\boldsymbol{A} \boldsymbol{x}
$$

with $\boldsymbol{A}$ being the design matrix, which contains the partial derivatives of $\phi(\boldsymbol{x})$ with respect to the unknown parameters $\left(\frac{\partial \phi(\boldsymbol{x})}{\partial \boldsymbol{x}}\right)$. Equation 6.8 can now be solved for $\boldsymbol{v}$, resulting in:

$$
\boldsymbol{v}=\boldsymbol{A} \boldsymbol{x}-\left(\boldsymbol{L}-\phi\left(\boldsymbol{x}_{0}\right)\right)=\boldsymbol{A} \boldsymbol{x}-\boldsymbol{l}
$$

with $\boldsymbol{l}=\boldsymbol{L}-\phi\left(\boldsymbol{x}_{0}\right)$ being the difference between measured and computed observations, the so-called residuals.

In the general least squares approach all measurements are given the same weight, not considering a-priori quality information for the observations which might be available. This is generally not suitable for a real-world scenario where not all the measurements will have equal quality and therefore equal error. In order to cope with this problem, and 
to furthermore get an error estimation for the unknown parameters, a stochastic model is employed by introducing the weighting matrix $\boldsymbol{P}$ :

$$
\boldsymbol{P}=\boldsymbol{Q}_{l l}^{-1}=\sigma_{0}^{2} \boldsymbol{\Sigma}_{l l}^{-1}
$$

where $\boldsymbol{Q}_{l l}$ denotes the cofactor matrix, $\sigma_{0}$ the a-priori variance factor and $\Sigma_{l l}$ the covariance matrix.

Different measurement weighting schemes have been introduced in GNSS processing over the years like e.g.

- Elevation angle: measurements at low elevation tend to have larger multipath and atmospheric delay errors than measurements at high elevation

- Signal-to-noise (SNR) ratio: decreased SNR serves as an indicator for e.g. high multipath activity,

which can be applied to find an appropriate formulation of the weighting matrix.

The actual (weighted) LSQ solution is found by a minimization of the term:

$$
v^{T} P v=\left(x^{T} A^{T}-l^{T}\right) P(A x-l)=x^{T} A^{T} P A x-x^{T} A^{T} P l-l^{T} P A x+l^{T} P l,
$$

which is achieved by calculating the first derivative with respect to $\boldsymbol{x}$ and setting it to zero:

$$
\frac{\partial \boldsymbol{v}^{\boldsymbol{T}} \boldsymbol{P} \boldsymbol{v}}{\partial \boldsymbol{x}}=2 \boldsymbol{A}^{\boldsymbol{T}} \boldsymbol{P} \boldsymbol{A} \boldsymbol{x}-2 \boldsymbol{A}^{\boldsymbol{T}} \boldsymbol{P l}=0
$$

This results in the normal equation:

$$
\left(A^{T} P A\right) x-A^{T} P l=N x-b
$$

with $\boldsymbol{N}=\boldsymbol{A}^{T} \boldsymbol{P} \boldsymbol{A}$ being the normal equation matrix and $\boldsymbol{b}=\boldsymbol{A}^{T} \boldsymbol{P l}$. If $\boldsymbol{N}$ is regular (i.e. invertible), solving for $\boldsymbol{x}$ gives the unknown parameter vector as:

$$
\boldsymbol{x}=\left(\boldsymbol{A}^{\boldsymbol{T}} \boldsymbol{P} \boldsymbol{A}\right)^{-1} \boldsymbol{A}^{\boldsymbol{T}} \boldsymbol{P l}=\boldsymbol{N}^{-1} \boldsymbol{b}
$$

An estimation of the accuracy of the derived parameters can be found via their variancecovariance matrix which is derived by inversion of the normal-equation matrix:

$$
Q_{x x}=N^{-1}
$$

Thereby the actual co-factors of the estimated parameters are found on the diagonal of $Q_{x x}$ 
In the concept of sequential LSQ (as utilized in the CSRS-PPP package, see Section 6.5), the estimated parameter vector serves an a-priori estimation of the unknown parameter vector in the next timestep. The same holds for the stochastic model of this approach as the variance-covariance matrix of the last timestep can be utilized as a-priori information in the next timestep.

\subsubsection{Kalman Filtering}

Another popular algorithm for parameter estimation is the Kalman Filter which was developed by Kalman (1960). It is widely used in signal processing, navigation, geosciences and numerous other scientific areas nowadays. Since it is also heavily used in this thesis, a more detailed description than for LSQ is given in the following.

In a general mathematical sense the algorithm belongs to a class of Bayesian estimation techniques, the Minimum Mean Square Error (MMSE) estimators. In more detail, it denotes the combination of sequential Linear MMSE (LMMSE) estimation with a so-called state-space model. This model accounts for the dynamic evolution of the system over time, which is a key difference/advantage compared to classical LSQ estimation. An observed set of parameters at a point in time $\mathrm{n}$ (the state vector) therefore is defined recursively by its prior state at $n-1$ and new observations coming in. The formulation of the Kalman filter algorithm outlined in the following is based on Hlawatsch (2019). The following equations define this state-space model for the Kalman filter algorithm:

$$
\begin{gathered}
\boldsymbol{y}[n]=\boldsymbol{H} \boldsymbol{x}[n]+\omega_{n} \\
\boldsymbol{x}[n]=\boldsymbol{A} \boldsymbol{x}[n-1]+\boldsymbol{B} \boldsymbol{u}[n],
\end{gathered}
$$

where $\boldsymbol{y}[n]$ denote the measured data (observations), $\boldsymbol{x}[n]$ the state vector at time $\mathrm{n}$ (or $\mathrm{n}-1$ respectively), $H$ the design matrix, $A$ the transition matrix, $B$ the control-input model matrix, $u[n]$ the control vector and $\omega_{n}$ the observation noise.

The algorithm is a sequential combination of a prediction and a correction step for the state vector and the error covariance matrix. Written down the formalism looks like:

$$
\begin{gathered}
\boldsymbol{x}[n \mid n-1]=\boldsymbol{A} \boldsymbol{x}[n-1 \mid n-1] \\
\boldsymbol{C}_{\boldsymbol{e}}[n \mid n-1]=\boldsymbol{A} \boldsymbol{C}_{\boldsymbol{e}}[n-1 \mid n-1] \boldsymbol{A}^{\boldsymbol{T}}+\boldsymbol{B}^{\boldsymbol{T}} \boldsymbol{B} \\
\boldsymbol{K}[n]=\boldsymbol{C}_{\boldsymbol{e}}[n \mid n-1] \boldsymbol{H}_{\boldsymbol{n}}^{\boldsymbol{T}}\left(\boldsymbol{H}_{\boldsymbol{n}} \boldsymbol{C}_{\boldsymbol{e}}[n \mid n-1] \boldsymbol{H}_{\boldsymbol{n}}^{\boldsymbol{T}} \boldsymbol{C}_{\boldsymbol{\omega}_{\boldsymbol{n}}}\right)^{-\mathbf{1}} \\
\boldsymbol{x}[n \mid n]=\boldsymbol{x}[n \mid n-1]+\boldsymbol{K}[n]\left(\boldsymbol{x}_{\boldsymbol{n}}-\boldsymbol{H}_{\boldsymbol{n}} \boldsymbol{x}[n \mid n-1]\right) \\
\boldsymbol{C}_{\boldsymbol{e}}[n \mid n]=\left(\boldsymbol{I}-\boldsymbol{K}[n] \boldsymbol{H}_{\boldsymbol{n}}\right) \boldsymbol{C}_{\boldsymbol{e}}[n \mid n-1]
\end{gathered}
$$


where $[n \mid n-1]$ denotes the index of a prediction and $[n \mid n]$ the index of a correction step. $C_{e}$ the error covariance matrix of the estimated parameters, $Q$ the covariance/correlation matrix of the control-input and $\boldsymbol{I}$ the identity matrix. $\boldsymbol{K}$ denotes the Kalman gain matrix which represents the key feature of the Kalman filter determining the correction step for the predicted parameters, based on the quality of the estimations $\left(\boldsymbol{C}_{\boldsymbol{e}}[n \mid n-1]\right)$ and of the incoming data $\left(\boldsymbol{C}_{\boldsymbol{\omega}_{n}}\right)$.

The presented algorithm can be extended for a number of other common features like coloured observation noise or the implementation of a non-linear state-space model, which is typically used in GNSS processing (e.g. in the GAMP software used in this study, see Section 6.7) and known as the Extended Kalman Filter (EKF).

\subsection{SEID}

As already mentioned before, the ionospheric delay on GNSS signals tracked by a SF receiver cannot be eliminated by forming linear combinations. A possible solution therefore is to correct for the ionospheric delay using TEC grids from global or regional ionosphere models. Then corrections can be applied to pseudorange and phase observations using formulas 4.16 .

As an alternative to this approach, the Satellite-specific Epoch-differenced lonospheric Delay model (SEID) was developed by Deng et al. (2009) at GeoForschungsZentrum (GFZ) Potsdam. It is based on the geometry-free LC $L_{4}$, the so-called ionospheric observation

$$
L_{4}=L_{1}-L_{2}
$$

which corresponds to the phase observation difference between $L_{1}$ and $L_{2}$ mainly governed by the ionospheric refraction.

Unfortunately this $L_{4}$ quantity has the major drawback of still containing the phase ambiguity parameter. In order to get rid of it, the algorithm uses epoch-differences of $L_{4}$, $\delta L_{4}$. For two consecutive time steps $i$ and $i+1$ this quantity reads

$$
\delta L_{4}(i)=L_{4}(i+1)-L_{4}(i)
$$

The ionospheric layer is represented by a single layer model, e.g. a thin shell with an altitude of about $300 \mathrm{~km}$ above Earth's surface. The spatial change of the delay is then expressed by the positions of the intercept pierce points (IPP) of the signal path and the layer (Deng et al. 2009). The basic assumption now is that this epoch-difference of a specific satellite, derived from DF stations, can be fitted to a linear function, e.g., a 
plane. Using the notation of Deng et al. (2009) this linear fit reads

$$
\delta L_{4}=\alpha_{0}+\alpha_{1} \lambda+\alpha_{2} \theta
$$

where $\theta$ is the latitude and $\lambda$ the longitude of their IPPs and $\alpha_{0}, \alpha_{1}$ and $\alpha_{2}$ are the model parameters to be determined.

From Equation 6.25 it is obvious that a minimum of three reference stations is required to derive those model parameters. In general a higher number of stations is beneficial to derive more accurate results and deal with possible data gaps at certain stations. In the case of four or more reference stations the parameters are estimated by means of a least-squares adjustment for each satellite at each epoch. This results in a different plane model for each satellite-epoch combination, which gives a hint on the high computational demand of the approach for larger datasets.

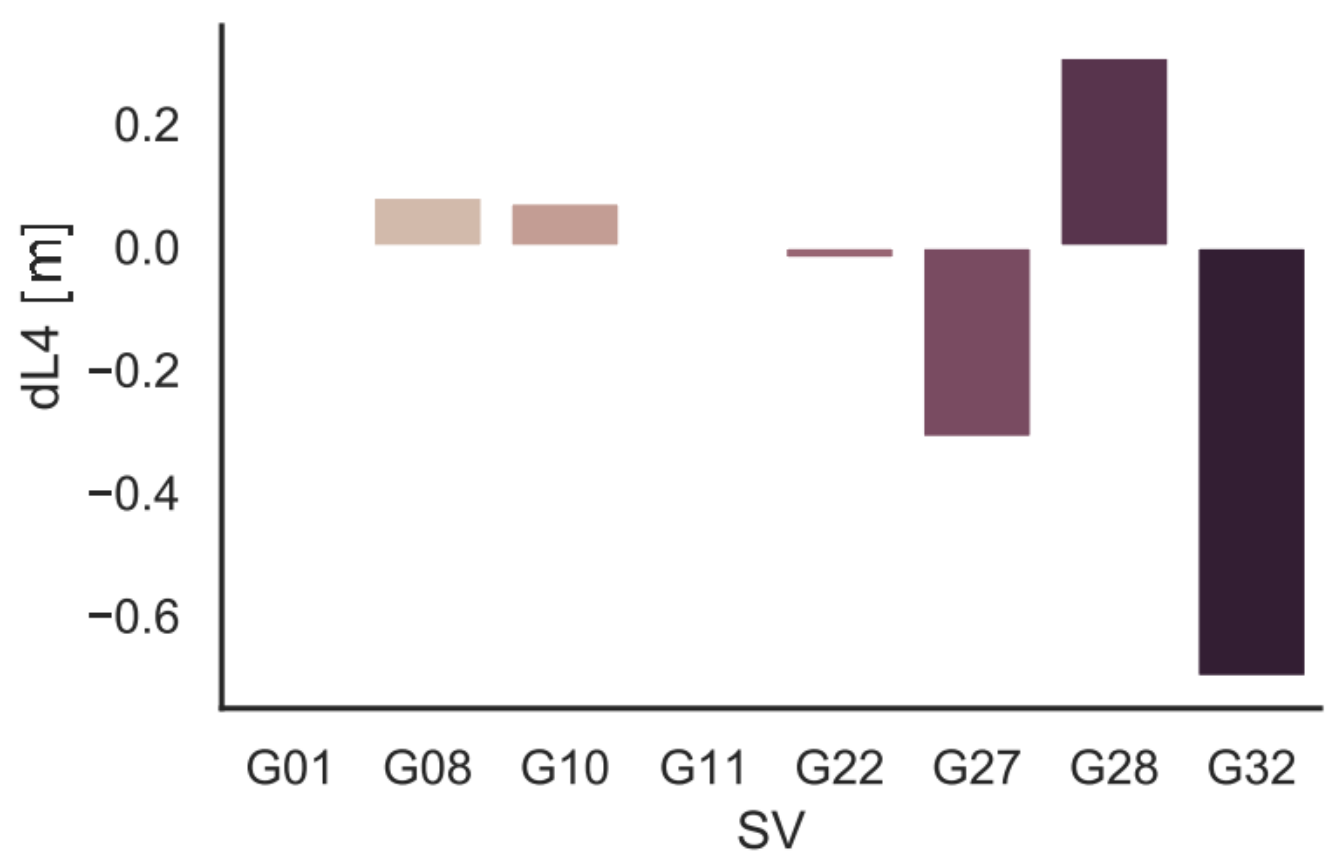

Figure 6.3: $\delta L_{4}$ values [m] for an example epoch and all GPS satellites processed within the SEID model. Larger values indicate possible outliers or cycle slips in the data of the respective satellite (e.g. G32 here).

Analysing the magnitudes of the computed $\delta L_{4}$ values, as shown in Figure 6.3, can be beneficial for detection of outliers and cycle slips affecting the utilized satellites at each epoch. In the processing carried out in this study a threshold of $\pm 0.5 \mathrm{~m}$ is applied for $\delta L_{4}$. If this threshold is exceeded, the corresponding satellite is excluded from the further processing. 
Using the generated model parameters at epoch $\mathrm{i}$, the epoch-differenced ionospheric correction of any SF receiver inside the reference network can be calculated. The correction for a specific epoch $k, \tilde{L}_{4}\left(i_{0}, k\right)$, is the sum of the epoch-differenced corrections from the first epoch $i_{0}$ on,

$$
\tilde{L}_{4}\left(i_{0}, k\right)=\sum_{i=i_{0}}^{k-1} \delta \tilde{L}_{4}(i, i+1) .
$$

By means of this quantity, a converted $L_{2}$ observation $\left(\tilde{L_{2}}\right)$ can be derived using

$$
\tilde{L_{2}}(k)=L_{1}(k)-\tilde{L}_{4}\left(i_{0}, k\right) .
$$

This basically represents the $L_{1}$ observation with the ionospheric delay adjusted to $L_{2}$.

Although they are usually significantly down-weighted or not used at all in PPP processing, pseudorange observations can be treated using the same procedure. The main difference to phase observations is the fact that here it is no longer necessary to operate with epoch-differences since no ambiguity parameter is present. Beside this the model is established in a similar way as for the carrier-phase,

$$
P_{4}=\beta_{0}+\beta_{1} \lambda+\beta_{2} \theta
$$

where $\beta_{0}, \beta_{1}$ and $\beta_{2}$ are the model parameters. $P_{2}$ is then calculated by

$$
P_{2}=P_{1}+P_{4}
$$

The original SF RINEX file is then extended by the converted $L_{2}$ and $P_{2}$ observations and can be used for DF processing in a software package of choice.

\subsubsection{Accuracy of SEID model}

Although a detailed accuracy analysis of the ionospheric corrections provided by the approach was not carried out, the most important findings from Deng et al. (2009) are summarized here.

Deng et al. (2009) analyzed the accuracy of both the ionospheric corrections $\tilde{L}_{4}$ and the STEC values derived using the SEID model. Therefore four reference stations were utilized, three of them for the computation of the model parameters and one of them was assumed to be a SF station. The interpolated $\tilde{L}_{4}$ was compared to computations of the GF-LC at this particular reference station. Denoting the standard deviation of the interpolated $\tilde{L_{4}}$ values as $\sigma_{\tilde{L}_{4}}$ and the standard deviation of the GF-LC values as $\sigma_{L_{4}}$, the following conclusions could be drawn from these comparisons (Deng et al. 2009): 
- If the SF station is located inside a triangle built up of the three reference stations, $\sigma_{\tilde{L_{4}}}$ has a value between $\sigma_{L_{4}} \cdot \sqrt{2 / 3}$ and $\sigma_{L_{4}} \cdot \sqrt{2}$. E.g. a $\sigma_{L_{4}}=3.3 \mathrm{~mm}$ gives a $\sigma_{\tilde{L_{4}}}=2.7-4.7 \mathrm{~mm}$.

- The interpolation error in STEC values derived with SEID equates to $\sim \pm 1 \mathrm{~mm}$ under average ionospheric conditions.

- Under abnormal ionospheric activity the STEC interpolation error is increased up to $\sim \pm 4 \mathrm{~mm}$ at low elevations.

Although still problems for describing sudden abnormal oscillations in ionospheric conditions exist, the results under average conditions indicate good performance of the approach and suitability of studies using SF GNSS like this one here. Nevertheless the high travelling speeds of the trains analysed here make some adaptations necessary which are described in the following section.

\subsubsection{Kinematic implementation}

Due to the highly kinematic nature of the train tracking data, SEID has to be executed in kinematic mode. Therefore a few adaptations have to be applied to the algorithm, the most important ones are listed below:

- Nearest reference stations change with the trains movement, therefore the list of used stations has to be updated in an appropriate time step (e.g. one hour)

- IPPs of DF and SF stations change with time/movement and have to be adjusted (more frequently than in a static scenario)

- Adoption of a weighting scheme based on the euclidean distance between train/receiver and reference station (the closer the reference station the larger the weight)

\subsection{Processing scheme: CSRS}

The CSRS-PPP online software was used to gain a first solution for the chosen days. The following information is based on Tétreault et al. (2005), who give a detailed overview of the software package. Here, only some important cornerstones and aspects of functionality and usage are outlined.

The online service for GPS users was introduced in 2003 by the Geodetic Survey Division of Natural Resources Canada and facilitates access to the Canadian Spatial Reference 
System (CSRS). CSRS-PPP processes GPS observations from single or dual-frequency GPS receivers operating in static or kinematic mode (Tétreault et al. 2005). User positions are estimated based on satellite orbits established in the International Terrestrial Reference Frame (ITRF).

The user online service of CSRS only needs RINEX data and a reference e-mail address as input. Positioning mode then can be chosen between static and kinematic and the reference frame for the output coordinates has to be selected (e.g. ITRF). Additionally an Ocean Tidal Loading (OTL) file can be introduced. Results are then returned to the given email address in form of text files containing position and ZTD estimates as well as their uncertainties.

The service was used to estimate position and ZTD from Greenlight RINEX data for the chosen days. The main processing/setup options can be found in Table 6.2.

\section{Feature}

\begin{tabular}{lc}
\hline Functional model & Undifferenced/Uncombined \\
Measurements & Code,Phase \\
Constellations & GPS, Glonass \\
Frequency & Single, Dual \\
Orbits/Clocks & IGS \\
lonosphere & GIM \\
Troposphere & VMF1 \\
Phase-Wind-Up & Corrected \\
Relativistic effects & 2nd order correction on clocks, gravitational time delay
\end{tabular}

Table 6.2: Default settings and features for the CSRS-PPP package.

\subsection{Processing scheme: PPP-Wizard}

Another suitable PPP software used in this research is the Precise Point Positioning With Integer and Zero-difference Ambiguity Resolution Demonstrator (PPP-Wizard). The software was developed at Centre National d'Etudes Spatiales (CNES) and aims at providing zero-difference AR for (RT-) PPP. Detailed information on the package can be found in Laurichesse and Privat (2015).

The main processing/setup options are outlined in Table 6.3. A main advantage of PPPWizard is the fact that SF data as well as (SEID-generated) DF data can be processed in a straightforward way. Furthermore the delivered package contains not only executables 
Feature

Functional model

Measurements

Constellations

Frequency

Orbits/Clocks

Ionosphere

Troposphere

Phase-Wind-Up
Undifferenced/Uncombined

Code,Phase,Doppler

GPS, Glonass for now, ongoing Galileo and Beidou

Single, Dual, Triple

EGNOS or IGS

EGNOS (SF) / IF-LC (DF)

Saastamoinen

Corrected

Relativistic effects 2nd order correction on clocks, gravitational time delay

Table 6.3: Default settings and features for the PPP-Wizard software.

but a fully-portable $\mathrm{C}++$ library which could be extended in the course of further research on this topic.

\subsection{Processing scheme: GAMP using SEID}

In order to gain yet another independent solution to compare with CSRS-PPP and PPPWizard, a combination of different software packages and algorithms was applied. The basic idea behind this approach is to generate a DF RINEX file out of the given SF file using the SEID model, described in Section 6.4. The generated DF observations are then processed in kinematic PPP mode using the GAMP software (Zhou et al. 2018). Emphasizing the combination of the SEID algorithm with the GAMP software for PPP processing, the approach is referred to as SGAMP in the following.

The concrete flow chart of the approach is shown in Figure 6.4. The main processing steps are the following:

1. Download suitable DF reference station data from the EPOSA network

2. Pre-process DF reference station data using goGPS

3. Apply SEID in kinematic mode to the SF data using the 5-6 nearest reference stations

4. Process the generated DF observations in kinematic PPP mode using GAMP The entire algorithm was implemented in Python. Here the Georinex package (https://pypi.org/project/georinex/) was used for easy reading of RINEX data. Parameter estimation was carried out with the scipy package (Virtanen et al. 2020). Further 


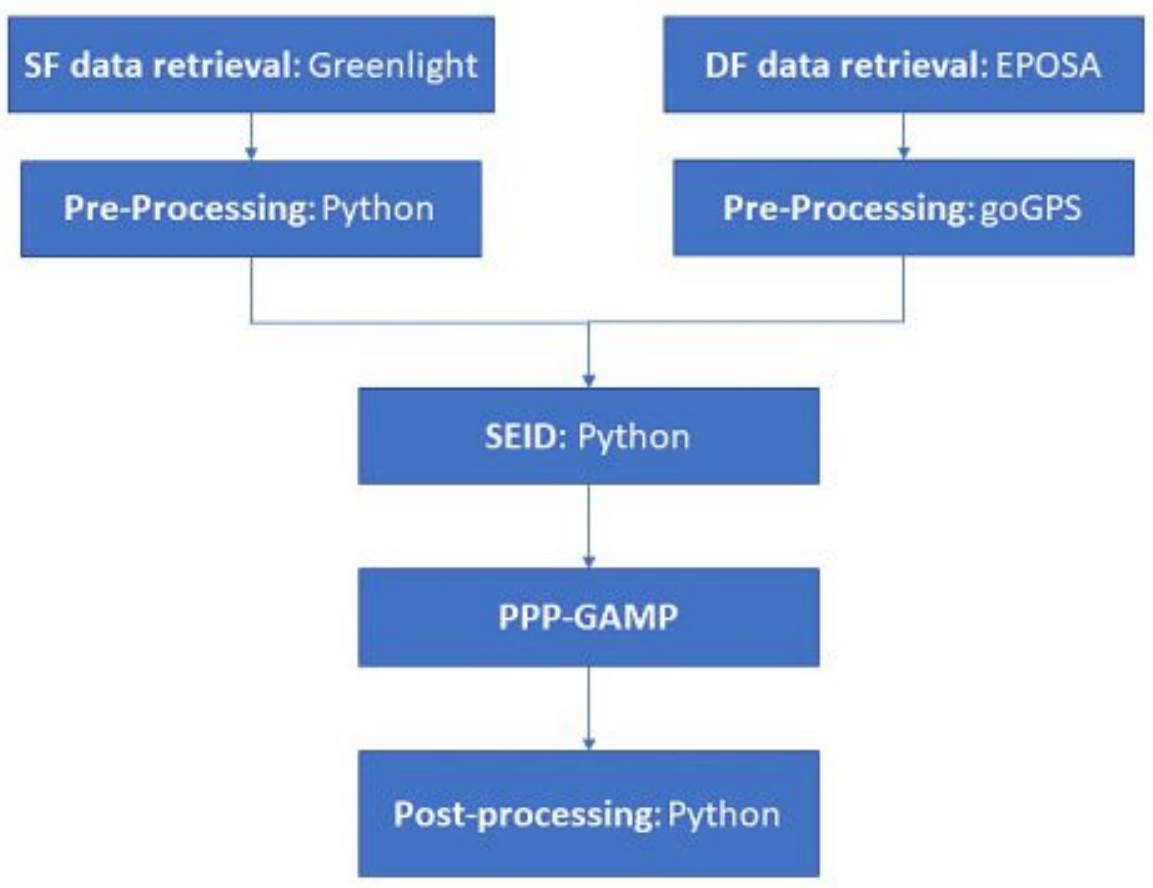

Figure 6.4: Flowchart of the SGAMP processing scheme as followed in this study.

details on the concrete implementation of the SEID model (e.g. chosen stations) are given in Section 8.3, where results of the test case are presented.

\subsubsection{GAMP}

The PPP software used for the SGAMP processing scheme was the GAMP software (Zhou et al. 2018), an open-source package developed at GFZ. It is capable of carrying out multi-GNSS PPP based on undifferenced and uncombined observations. The software is a secondary development which is based on RTKlib (Takasu and Yasuda 2009), but with many improvements in terms of cycle slip detection, receiver clock jump repair, or handling of GLONASS pseudorange inter-frequency biases. An overview of the processing capabilities of GAMP are given in Table 6.4.

\subsection{Processing scheme: Height-constrained PPP}

Parameter constraints have been extensively used in GNSS processing (e.g. Xu (2016), Suya (2019), Gao et al. (2017)) as well as sensor fusion algorithms (Huang et al. (2018), Zhu et al. (2020)). Even the case of static PPP processing represents a constrained solution as the coordinate solution is to some degree constrained to a stationary site. In this study, a tight constraint on the estimated height coordinate is used to support the 
Feature

Functional model

Measurements

Constellations

Frequency

Orbits/Clocks

lonosphere

Troposphere

Phase-Wind-Up
Undifferenced/Uncombined

Code,Phase

GPS, Glonass, Galileo and Beidou

Single, Dual

Final/rapid/ultra-rapid

IF-LC (dual) or lono-model

NMF or GMF

Corrected

Relativistic effects 2nd order correction on clocks, gravitational time delay

Table 6.4: Default settings and features for the GAMP PPP software.

tropospheric estimation. The idea behind this approach is to achieve significant decorrelation between ZTD and height estimates by using precise height information from a GNSS-independent source. Therefore heights from an existing database of railway tracks all over Austria, established by classical surveying methods (e.g. levelling), are used here. The database was thankfully provided by ÖBB. For more detailed information concerning this dataset see Section 6.1.2. In the following the actual implementation of the height-constraint is shortly introduced.

The basic procedure is just a simple correction of the height estimate in the Kalman Filter, by using the height provided at the nearest point in the track database. This nearest point is determined by searching the closest point in the database to the state estimate of the 2D-coordinates (prediction step in the filter), based on euclidean distance.

Additionally, a height offset has to be applied since the coordinates of the database points do not refer to the GNSS antenna but to (reference-) pylons along the railway tracks. Since no concrete values for this offset were available, one can either use the pylon heights directly (this will result in a more loosely constraint) or set a default value. The latter approach was carried out here with a default offset determined by using the median of calculated deviation between GNSS heights from CSRS-PPP (lowest noise of all PPP solutions, see Chapter 8 for detailed results) and pylons for three of the four test cases (where track database was available). This equates to an offset of $\sim 4$ meters which was applied to the database heights.

Moreover one can decide when to apply the correction: (1) always or (2) based on an accuracy criterion relative to the track database. In this study, the correction/replacement 
of the height estimate is applied when the following condition is met:

$$
\left|H_{G N S S}-\left(H_{R D B}+d H\right)\right|>0.1 m
$$

where $H_{G N S S}$ and $H_{R D B}$ are the ellipsoidal heights from GNSS and the track database respectively and $d H$ is the applied offset between pylon and antenna.

\subsection{Filtering methods and performance metrics}

Despite the usage of an appropriate parameter estimation method (e.g. Kalman Filter or LSQ), the PPP solution can show a quite large amount of noise in the estimates. This is present especially within the convergence time of the filters in the beginning of processing or after data gaps where the solution was lost. In order to remove this noise and also quantify its magnitude, a filtering (i.e. a smoothing) of the parameter estimates is carried out.

Additionally some common statistical measures, which are used to describe the results shown in Chapter 8, are presented. They serve as performance indicators in case of the noise level analysis of the PPP solutions and the validation of ZTD solutions (as described in Chapter 7).

Furthermore it should be noted here that for every parameter estimation (i.e. PPP processing) error estimates are available from the covariance matrix (as detailed in Section 6.3). However, these formal errors are often much too optimistic when compared to results of a validation using independent reference data. Moreover, some PPP software packages (as CSRS-PPP or GAMP in this case) do not even provide such formal errors for ZTD estimates. Therefore a detailed analysis of these formal errors was omitted and instead only comparisons with smoothed solutions (for noise analysis/filtering) and reference datasets (ZTD derived from ERA5) are presented for this study.

\subsubsection{Moving average filtering}

The Moving Average (MA) filter is a simple Low Pass FIR (Finite Impulse Response) filter which is commonly used for smoothing timeseries of sampled data. It takes a finite number of samples (denotes as $N$ here) of input at a time and takes the average of those $N$-samples and produces a single output point. This denotes a very simple LPF (Low Pass Filter) structure to filter unwanted noisy components from the analysed data. As the filter length increases (the parameter $N$ ) the smoothness of the output increases, whereas the sharp transitions in the data are made increasingly blunt. This implies that 
this filter has excellent time domain response but a poor frequency response. Important features of the MA filter can be summarized as follows

- The algorithm computes the average of $N$-points and produces a single output point

- Due to the computation/calculations involved, the filter introduces a definite amount of delay $(N / 2)$

- The filter acts as a Low Pass Filter (with poor frequency domain response and a good time domain response).

The mathematical formulation of a $N$-point discrete-time moving average filter with input represented by the vector $\mathrm{x}$ and the averaged output vector reads

$$
y[n]=\frac{1}{N} \sum_{k=0}^{N-1} x[n-k]
$$

As already indicated above, this calculation introduces a delay of $N / 2$ in the timeseries which has to be accounted for. In the results shown later, a time shift by $N / 2$ is carried out in order to get a filtered estimation of the right (corresponding) timeseries entry.

\subsubsection{Error metrics for performance evaluation}

This section introduces the utilized statistical measures for the quality assessment/validation and of the conducted case studies shown in the next chapters. All measures shown here are well-known and frequently used in geosciences, therefore only a short description and analysis of advantages/disadvantages is given here. For detailed information on properties, performance features and other feasible measures, Wilks (2011) provides an extensive discussion.

In the following five basic scalar measures of accuracy, used in this study, are introduced. Although many more are available, all validation results (see Chapter 8 ) are expressed in terms of those due to reasons like easy understanding and implementation.

1. BIAS:

$$
B I A S=\frac{1}{n} \cdot \sum_{k=1}^{n} y_{k}-r_{k}
$$

The Bias (or Mean Error) is given as the mean difference between the observed $\left(y_{k}\right)$ and reference values $\left(r_{k}\right)$ of a timeseries. Its unit is the same as for the analysed parameters. 
2. Standard deviation: The standard deviation of a time series $y$ is defined as the square root of its variance:

$$
\sigma=\sqrt{\frac{1}{n-1} \sum_{k=1}^{n}\left(y_{k}-\bar{y}\right)^{2}}
$$

3. Root Mean Square Error: The Root Mean Square Error (RMSE) denotes the square root of the Mean Squared Error (MSE), which is the average squared difference between the reference and observation pairs:

$$
R M S E=\sqrt{M S E}=\sqrt{\frac{1}{n} \cdot \sum_{k=1}^{n}\left(y_{k}-r_{k}\right)^{2}}
$$

4. Median: The Median is defined as the mid-element of a monotonically increasing time series and represents the value separating the higher half from the lower half of a data sample. Main advantage of the median compared to the arithmetic mean is its robustness to outliers in the dataset.

$$
\begin{gathered}
\text { median }_{\text {odd }}=y\left(\frac{n+1}{2}\right) \\
\text { median }_{\text {even }}=\frac{y\left(\frac{n}{2}\right)+y\left(\frac{n}{2}+1\right)}{2}
\end{gathered}
$$

5. Pearson correlation coefficient: The Pearson correlation coefficient $R$ can be calculated as:

$$
R(X, Y)=\frac{\operatorname{Cov}(X, Y)}{\sqrt{\operatorname{Var}(X)} \sqrt{\operatorname{Var}(Y)}},
$$

where $X$ and $Y$ are random variables. It is a unitless quantity and takes values from -1 to +1 . 


\section{Chapter 7}

\section{ZTD validation and assimilation}

The quality of ZTD estimations derived using the GNSS processing strategies outlined in Chapter 6 needs to be assessed using independent data. This can be achieved by a comparison to different datasets, including:

- ZTD calculated from NWP forecast/analysis fields

- ZTD calculated from reanalysis products

This study utilizes the latter method to get a first quality estimation for the derived ZTD results.

The following chapter is structured as follows:

At first it introduces the independent data sources and the methodology behind the ZTD calculation from these data. Results of these comparisons for all test cases are shown in Chapter 8.

Secondly, an introduction to the basic principle of DA is given, and some of the most prominent techniques for DA, like different variational methods, are introduced.

Afterwards, the current state-of-the-art of assimilation of GNSS data in NWP is analysed.

Finally the proposed approach for DA within this study is presented, introducing the utilized model, the techniques used and the methods for verification of the results. 


\begin{tabular}{lcccccc|}
\hline Reanalysis & Period covered & Grid resolution & Assimilation scheme & IFS model cycle (year) \\
\hline FGGE & 1979 & $208 \mathrm{~km}$ & OI & $(1980)$ \\
ERA-15 & $1979-1994$ & $125 \mathrm{~km}$ & OI & $13 \mathrm{r} 4(1995)$ \\
ERA-40 & $1957-2002$ & $125 \mathrm{~km}$ & 3D-Var & $23 \mathrm{r} 4(2001)$ \\
ERA-Interim & $1979-2019$ & $80 \mathrm{~km}$ & 4D-Var & $31 \mathrm{r} 2(2006)$ \\
ERA5 & 1950-present & $31 \mathrm{~km}$ & 4D-Var & $41 \mathrm{r} 2(2016)$
\end{tabular}

Table 7.1: Detailed information on the product line of reanalyses produced by ECMWF.

\subsection{Validation using ERA5 reanalysis products}

The validation of GNSS-ZTD estimates is carried out using the ERA5 reanalysis dataset (Hersbach et al. 2020), provided by the European Centre for Medium-Range Weather Forecasts (ECMWF). This section provides some basic details on the dataset and the methods utilized to derive ZTD reference values from it. Most information is based on Hersbach et al. (2020), who provide a detailed description of the products available and the methodology of its production.

\subsubsection{ERA5}

The ERA5 reanalysis is the newest product of the ERA family produced by ECMWF. This new reanalysis replaced the ERA-Interim reanalysis (spanning 1979 onwards) which was initiated in 2006. ERA5 is based on the Integrated Forecasting System (IFS) Cy41r2 which was operational in 2016 (Hersbach et al. 2020). The dataset provides hourly estimates of a large number of atmospheric, land, and oceanic climate variables. The data is provided at $30 \mathrm{~km}$ horizontal resolution and 37 vertical levels from the surface up to a height of $80 \mathrm{~km}$.

The first parts of the ERA5 dataset are available for public use (1979 to within 5 days of real time) since 2019. In its final state, the ERA5 reanalysis will represent a detailed record of the global atmosphere, land surface and ocean from 1950 till today.

Compared to its predecessor ERA-Interim, a significantly enhanced horizontal resolution of $31 \mathrm{~km}$ (compared to $80 \mathrm{~km}$ for ERA-Interim) is available for ERA5 (see also Table 7.1). An example visualisation of $2 m$-temperature ERA5 data for January 2016 is shown in Figure 7.1. 


\subsubsection{ZTD calculation from ERA fields}

The ZTD calculation from the ERA5 reanalysis fields follows the approach of Wilgan and Geiger (2018), who used temperature, pressure and specific humidity fields from the NWM COSMO-1, which is the operational NWM at MeteoSwiss, specifically designed to produce the weather forecast in the challenging Alpine region (MeteoSwiss 2020). The approach uses the formulation of Essen and Froome (1951) for the total refractivity

$$
N_{t o t}=k_{1} \frac{p}{T}-k_{2} \frac{e}{T}+k_{3} \frac{e}{T^{2}}
$$

where $p$ denotes pressure, $T$ the temperature, $e$ the partial water vapour pressure and $k_{1}=77.689 \mathrm{KhPa}^{-1}, k_{2}=71.2952 \mathrm{KhPa}^{-1}$ and $k_{3}=375463 \mathrm{KhPa}^{-1}$ the optimal average refractivity coefficients from Rüeger (2002), empirically determined for the L-band frequencies.

Corresponding ERA fields for pressure, temperature and specific humidity (which allows for computation of $e$, see Chapter 3) are obtained from https://cds.climate.copernicus.eu/. This way 4D fields of refractivity are computed which allow for the computation of ZTDs through vertical integration, as shown in Equation 7.2.

$$
Z T D_{E R A}=10^{-6} \cdot \int N_{t o t},
$$

where $Z T D_{E R A}$ represents the resulting 3D ZTD field and $N_{\text {tot }}$ the $4 \mathrm{D}$ refractivity field calculated from ERA5 fields using Equation 7.1.

In the case of discrete data as given here for ERA5, Equation 7.2 can be approximated by Equation 7.3:

$$
Z T D_{E R A}=10^{-6} \cdot \sum_{i=1}^{N L-1} \frac{N_{i}+N_{i+1}}{2} \cdot \delta s_{i},
$$

where $N_{i}$ represents the total refractivity at the i-th vertical level, $N L$ is the number of vertical levels of ERA 5 and $\delta s_{i}$ represents the geometric distance between $\mathrm{i}$-th and $(\mathrm{i}+1)$-th layer.

Furthermore the delay at the top level $N L$ is derived using Equation 7.4 (Saastamoinen 1972):

$$
Z T D_{N L}=0.002277 \cdot\left(p_{N L}+\left(\frac{1255}{T_{N L}}+0.05\right) \cdot e_{N L}\right)
$$

where $p_{N L}, T_{N L}$ and $e_{N L}$ represent the respective parameters at the highest vertical level of ERA5. The total ZTD is then given by Equation 7.5:

$$
Z T D_{\text {total }}=Z T D_{E R A}+Z T D_{N L}
$$


The actual ZTD value for a given point on the train-trajectory is found via linear interpolation of the 3D ZT $D_{\text {total }}$ field.

\subsubsection{Quality of ERA5-derived ZTD}

Since ERA5 is one of the newest atmospheric reanalyses, not many studies have already assessed the quality of ZTD values calculated from its data. Validation of temperature, wind and humidity data before the actual release of ERA5 already indicate increased performance since comparisons with radiosonde show an improved fit for those parameters, at least in the troposphere (Hersbach et al. 2020).

First assessments of ZTD were presented by Jiang et al. (2020), who evaluated the performance of ERA5-derived ZTD against GNSS-ZTD over China using data from 219 GNSS stations for the period 2015 to 2016 . They found an average bias and RMS between ERA5-ZTD and GNSS of $2.97 \mathrm{~mm}$ and $11.49 \mathrm{~mm}$, respectively.

Ssenyunzi et al. (2020) investigated the performance of ERA5 for retrieval of PWV over the East African tropical region and found RMS errors between GNSS- and ERA5-derived PWV values are in the range of $1.35 \mathrm{~mm}$ to $2.25 \mathrm{~mm}$ with the overall average value of $1.66 \mathrm{~mm}$.

Overall, these studies indicate comparable results for GNSS and ERA5 regarding the investigated quantities. Moreover the ZTD/PWV estimates from GNSS used in the studies mentioned above were derived from high-quality DF GNSS data. This encourages the use of ERA5 as the reference data source for the performance assessment of SF GNSS-ZTD estimates as carried out in this study.

Nevertheless it should be mentioned here that NWP-derived data like the ERA5 reanalysis are an independent source of ZTD, which implies that consistency with GNSS-derived ZTD cannot always be expected. In particular, station and NWP-specific biases can occur, which are justified by a deficiency of the NWP orography representation (Kačmařík et al. 2017). Therefore, when comparing GNSS ZTD to NWP model products, a bias should not be considered as a quality indicator, because station-specific GNSS ZTD biases are removed prior to ZTD assimilation (Mile et al. 2019). 

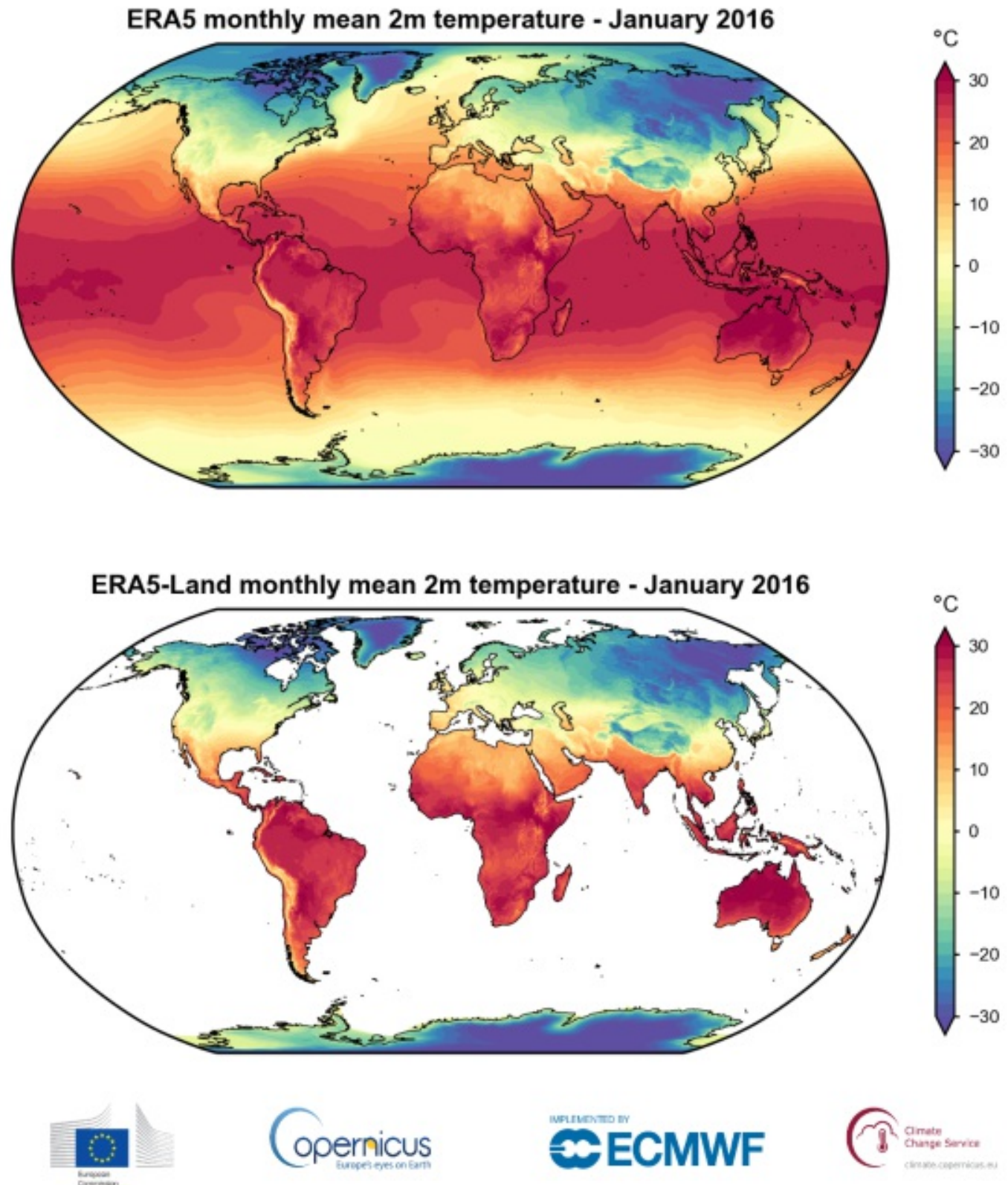

Figure 7.1: ERA5 global grid of 2m-temperature for January 2016. Top: full reanalysis, bottom: ERA5-Land reanalysis. Taken from: https://climate.copernicus.eu/climate-reanalysis 


\subsection{Basics of data assimilation}

NWP denotes an initial value problem and therefore strongly relies on the quality of the initial model state. The process of acquiring the best possible initial state is called data assimilation. The current section provides a small overview of DA concepts and methods as implemented in NWP models.

In a strictly mathematical sense DA denotes the process of obtaining the statistically best combination between model state and the available observations at a certain period of time. This includes considering the uncertainty of both observations and model predictions in the process. Common techniques to achieve this are variational methods like 3D- or 4D-Var, different types of Kalman Filtering or nudging. For most cases, like the variational methods, this best initial state is derived by a combination of observations with a model field of the observed variable, just before the start of the next forecast run. This principle is indicated in Figure 7.2 for a 24-hour assimilation window. The

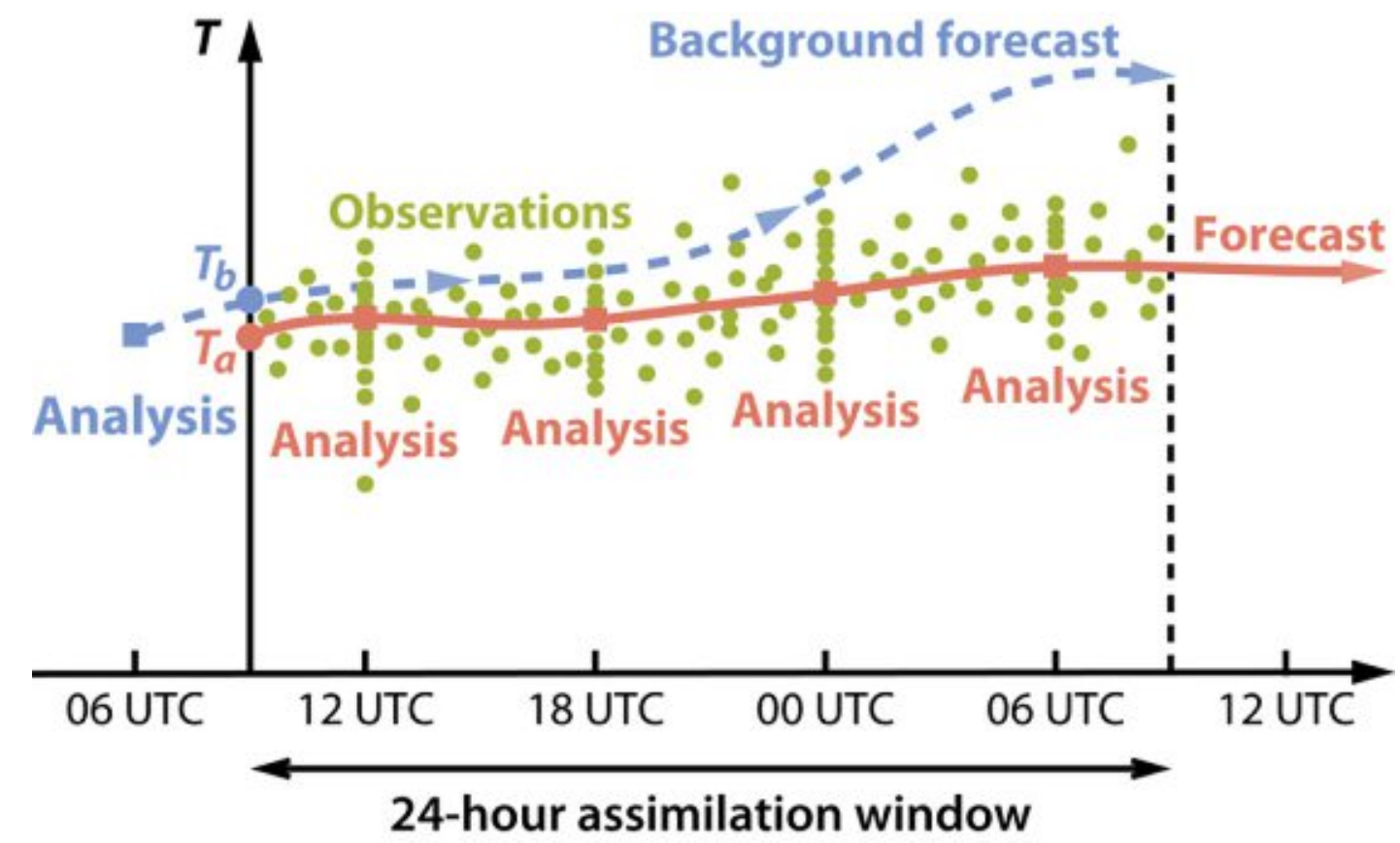

Figure 7.2: The basic principle of DA and its main components outlined for the temperature ( $T$, y-axis) parameter and a 24-hour assimilation window ( $x$-axis): background forecast (blue), observations (green) and analysis (red).

model field denotes a short-term forecast from the previous forecast cycle which is called first guess or background forecast. Based on model and observation error estimates the so-called analysis is calculated, which serves as the initial state for the next forecast step.

The optimal analysis is obtained by minimizing a cost function measuring the misfit 
between observation and prediction by a non-linear model, which has the basic form shown in Equation 7.6

$$
\boldsymbol{J}(\boldsymbol{x})=\left(\boldsymbol{x}-\boldsymbol{x}_{\boldsymbol{b}}\right)^{\boldsymbol{T}} \boldsymbol{B}^{-1}\left(\boldsymbol{x}-\boldsymbol{x}_{\boldsymbol{b}}\right)+\frac{1}{2}\left(\boldsymbol{H}[\boldsymbol{x}]-\boldsymbol{y}^{\boldsymbol{T}}\right) \boldsymbol{R}^{-1}(\boldsymbol{H}[\boldsymbol{x}]-\boldsymbol{y}) .
$$

In the above equation, $\boldsymbol{x}$ represents the analysis vector (the initial atmospheric state), $\boldsymbol{x}_{\boldsymbol{b}}$ the forecast background vector and $\boldsymbol{y}$ the observation vector. $\boldsymbol{H}$ denotes the observation operator transforming model variables into observation space. $\boldsymbol{R}$ denotes the observation error covariance matrix and $\boldsymbol{B}$ the background error covariance matrix which contain information about the errors in the observations and the background field and their respective correlations.

The next section introduces some prominent DA techniques which are based on Equation 7.6.

\subsubsection{DA techniques}

The most common analysis methods used by current NWM include:

- Variational methods (3D-Var and 4D-Var)

- Ensemble Kalman Filter (EnKF)

Typical implementations of variational methods rely on single deterministic forecasts. In contrast to this, the EnKF uses an ensemble approach to evaluate and apply assimilation increments. Major advantages of this technique include ease of implementation (unlike 4D-Var, EnKF does not require an adjoint model) and the generation of useful estimates of analysis uncertainties, which are difficult to obtain when using variational techniques with single forecasts (Fujiwara et al. 2017). A conceptual overview of the most prominent methods is given in Figure 7.3. 
(a) 3D-Var (increments calculated and applied at analysis times)

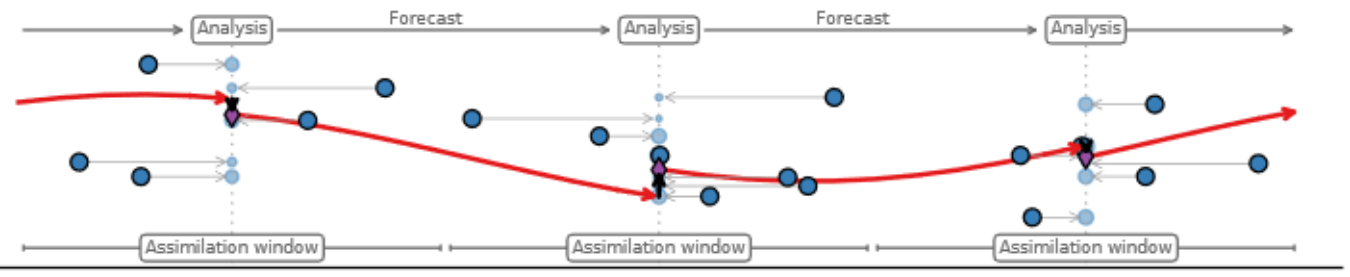

(b) 3D-FGAT (increments estimated at observation times but applied at analysis times)

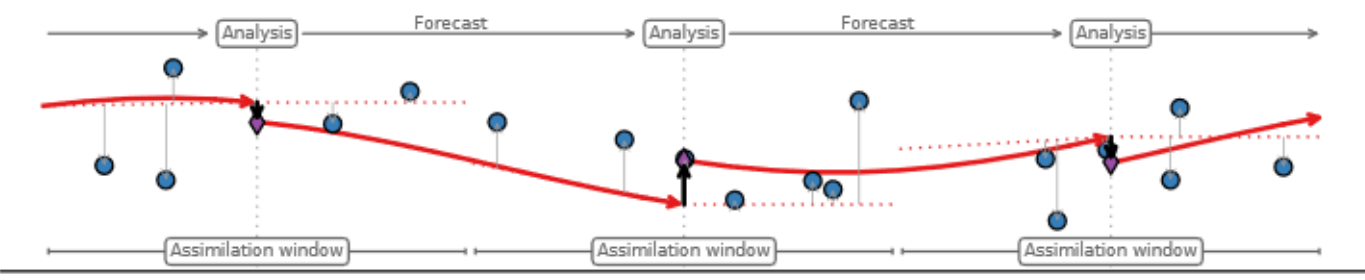

(c) 4D-Var (iteratively estimate increments for full window and adjust initial state)

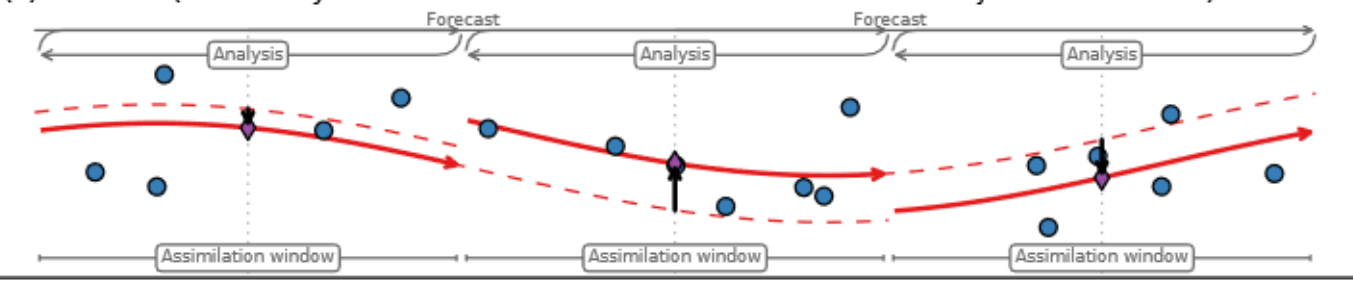

(d) EnKF (increment applied as a Bayesian update to the posterior forecast ensemble)

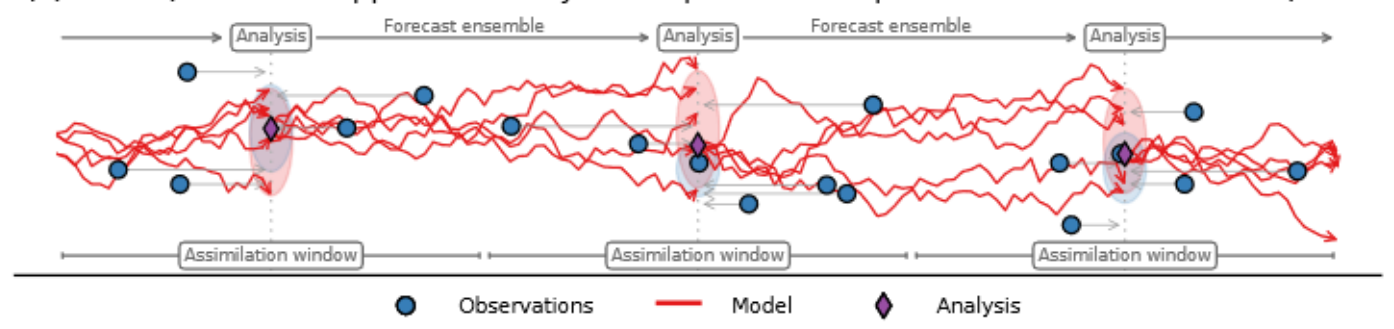

Figure 7.3: Simplified schematic representations of four data assimilation strategies frequently-used in NWP:(a) 3D-Var,(b) 3D-FGAT,(c) incremental 4D-Var, and (d) EnKF. Blue circles represent observations, red lines represent model trajectories, and purple diamonds indicate analyses. The dotted red lines in(b)represent linearly interpolated or extrapolated forecast values used to estimate increments at observation times. The dashed red lines in(c)represent the initial forecast, prior to iterative adjustments. These illustrations are conceptual and do not accurately reflect the much more complex strategies used by reanalysis systems. Taken from Fujiwara et al. (2017) 


\subsection{Assimilation of GNSS data in NWP}

The following section introduces the current state-of-the-art and developments in the field of GNSS DA in NWP. A lot of information presented here was already outlined in Aichinger-Rosenberger (2018).

Nowadays ZTD assimilation is possible for all operational models in Europe, with the exception of COSMO, which uses the related measure integrated water vapour (IWV). The usage of GNSS for investigation on the water vapour field has been the topic of several studies over the last couple of years. Different weather phenomena have been investigated, focusing on short-term forecasts and severe events. A number of authors studied the relationship between the water vapour field and the life cycle/intensity of precipitation systems (Guerova et al. 2016). Yan et al. (2009) showed that the assimilation of ZTD data in the AROME model induces changes in the vertical structure of low-to-middle tropospheric water vapour. These changes are consistent with measurements from radiosonde and airborne lidar. Seco et al. (2012) found a relationship between IWV (or rather ZTD) and precipitation intensity in the north of Spain during the period 2002-2010. Furthermore evidence that water vapour plays a key part as precursor in the initiation of local convection was found by van Baelen et al. (2011). Graham et al. (2012) showed that the large transfer of water vapour amount (e.g. between a mountainous area and the flatland) in convective systems initiated by orography can be detected in IWV/ ZTD. The link between the moisture field and lightning and thunderstorm activity was established by de Haan (2008). This was used by Brenot et al. (2013) to identify conditions for deep convection and provide a nowcasting warning system. 


\subsection{Assimilation and verification methodology}

This following section describes the assimilation and verification approach used within this study. Due to the special nature of the GNSS data and their novelty in usage for NWP-DA drag some simplifications and adaptations to common approaches with them.

\subsubsection{Model description and setup}

The Weather Research and Forecasting (WRF) Model is utilized for this study. It is an atmospheric modelling system designed for both research and numerical weather prediction (Skamarock et al. 2019). As an open-source community model it has been adopted for research at universities and governmental laboratories, for operational forecasting by governmental and private entities, and for commercial applications by industry. The development of WRF started around the years 1995-2000 with the goals being to build a system shared by research and operations and to create a next-generation NWP capability. The resulting modelling system is nowadays a common platform which can be used and developed further by a broad research community.

Figure 7.4 shows the system components of the advanced research version of WRF used for this study. The main focus in this case lies on the WRFDA Data Assimilation module (Barker et al. 2012), which performs all the necessary steps attached to the ZTD assimilation. Main goal of all the assimilation tests carried out here is the assessment of the number of observations providing sufficient accuracy to be processed by the WRFDA module (i.e. passing its quality check). This actually represents yet another type of validation for the GNSS-ZTD estimates since they are compared to values calculated by the background state/forecast of the model.

\section{ZTD operator}

The WRFDA system used in this study provides variational 3D/4D as well as hybrid variational ensemble algorithms (Barker et al. 2012). Currently, it supports the assimilation of surface, radiosonde, aircraft, wind profile observations, as well as atmospheric motion vectors, radar reflectivity, spectrometric, GPS radio occultation and GPS ground-based data (Rohm et al. 2019). A specific observation operator, the GPSPW operator defines the forward, tangent linear and adjoint of $\mathrm{H}$ for the 4D-Var and 3D-Var case for ZTD and PW. The ZTD forward operator $\mathrm{H}$ reads as follows (Vedel and Huang 2004) with 


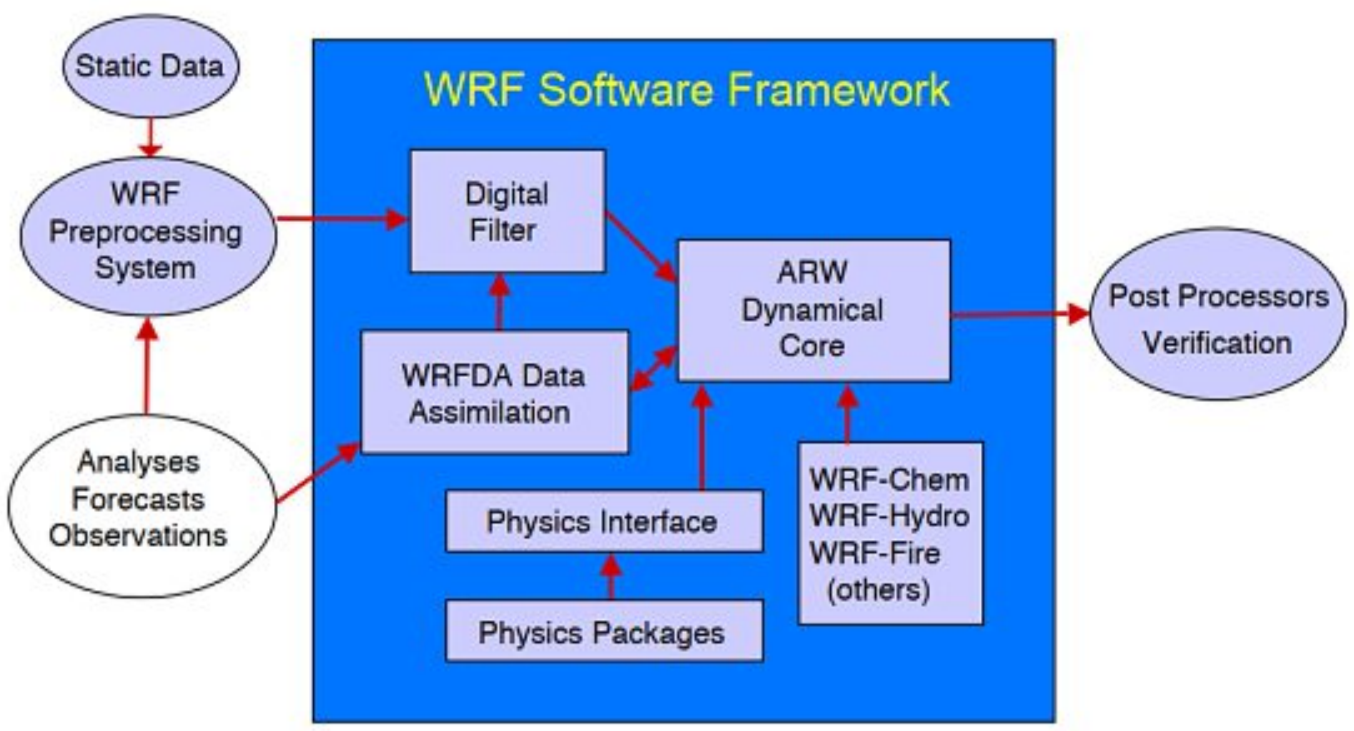

Figure 7.4: System components of Advanced Research WRF, taken from Barker et al. (2012).

further corrections made by Yong-Run Guo and presented in Rohm et al. (2019):

$$
\begin{gathered}
Z T D(i, j)=Z H D(i, j)+Z W D(i, j) \\
Z W D(i, j)=\sum_{k=k t e}^{k=k t s}\left(\frac{\left(w d k_{1} p(i, j, k) q(i, j, k)\right.}{t(i, j, k)}+\frac{w d k_{3} p(i, j, k) q(i, j, k)}{t 2(i, j, k)}\right) \frac{\delta h}{a_{e w}}
\end{gathered}
$$

where $i, j$ and $k$ are the model node indices, $p$ is pressure, $q$ specific humidity, $t$ temperature and $\delta h$ is the height difference between two consecutive model layers. Furthermore $a_{\text {ew }}=0.622$, as well as $w d k 1=2.21 \cdot 10^{-7}$ and $w d k_{3}=3.73 \cdot 10^{-3}$ are compressibility constants. $Z H D$ is computed with the Saastamoinen model as given in Equation 4.22.

The ZTD operator described above was used in the WRFDA module for the assimilation tests carried out for this study. Results achieved for two test tracks/cases can be found in Chapter 8, Section 8.7. 


\section{Chapter 8}

\section{Results}

Chapter 8 presents the main results of this study in the form of four case studies (i.e. test cases). The case studies comprise various train tracks as detailed in Section 8.1 and summarized in Table 8.1. The data on which the presented results are based upon are raw GNSS observations with an update rate of $1 \mathrm{~Hz}$. They were gathered in the course of the Greenlight project (see Section 6.1.1) and thankfully provided by ÖBB.

At first, the test cases (travelling routes and dates) and the used processing schemes for each case as well as the applied processing settings are detailed in Section 8.1.

Main results of the investigated case studies are presented and discussed in Sections 8.2, 8.3, 8.4 and 8.5.

Furthermore, Section 8.6 provides some additional ZTD comparisons which aim to investigate the sensitivity of the ZTD solution to changes in certain aspects of the processing settings (i.e. changes to the default settings introduced in Section 8.1.2).

Finally, Section 8.7 discusses the findings of the first assimilation tests carried out using ZTD time series from two of the conducted case studies.

\subsection{Overview of conducted case studies}

In the following, an overview of the conducted case studies and the used processing settings is given. For the analysed test cases (Sections 8.2 - 8.5) the presentation of the results is split into three sections:

1. Raw data quality: At first, some indicators of the quality of the obtained raw GNSS data are shown in this first section. The presented parameters are the CMC observables (as introduced in Section 6.2), the number of visible satellites as 
well as GDOP values. This section aims for a first estimation of the observation quality and the identification of possibly critical sections along the investigated tracks where degradation of the PPP solution can be expected.

2. PPP noise analysis: This section presents an analysis of PPP results (in terms of height coordinates and ZTD) from two different processing schemes (CSRS-PPP and PPP-Wizard). Main goal is an estimation of the noise level of the respective PPP solution for the height coordinate and ZTD. Therefore, a comparison between actual software output against a smoothed (i.e. filtered) solution is carried out for these two parameters. The smoothed solution is computed using an MA-filter, as detailed in Chapter 6, Section 6.9.1. The time window of the MA-filter is chosen based on the following criteria:

- Adequate smoothing effect: smooth noise in the parameters as much as possible without loosing information on variations originating from train movement. Therefore a mean travelling speed of $100 \mathrm{~km} / \mathrm{h}$ is assumed.

- Output interval to be used later for NWP assimilation: Delivering data for assimilation every second is not suitable and would not represent an added value to weather forecasts since tropospheric conditions do not change this fast. Furthermore the amounts of data and thus the computational effort would be huge. Therefore a good compromise has to be found in order to use as much valuable data as possible in a given DA setup.

Considering these criteria listed above, a time window of five minutes was chosen. This corresponds to a mean travel distance of $\sim 8 \mathrm{~km}$ which was assumed not to show too large variations in height or ZTD along the track (at least under average conditions, might not be true in highly mountainous regions) in order to filter out only noise components.

3. ZTD validation: In a second step, the ZTD estimates derived from PPP processing (referred to as GNSS-ZTD) are validated using ZTD calculated from ERA5 reanalysis data (referred to as ERA5-ZTD) as outlined in Chapter 7. Here deviations from ERA5-ZTD are compared between different schemes. Results are analysed in terms of the standard error measures outlined in Section 6.9.2.

\subsubsection{Test cases}

Four different test cases were investigated in the course of this study. Their main characteristics in terms of travelling route are summarized in the following descriptions 
and in Table 8.1.

CS1 uses data from a train travelling on the route Salzburg - Vienna airport. Challenging conditions are experienced due to a number of urban areas and tunnels along the track, which degrade the PPP solution or even account for a complete loss of the solution.

CS2 covers the route Salzburg - Klagenfurt which includes a lot of mountainous areas and ultimately a passing of the main alpine ridge as well as tunnels and some urban environments.

CS3 and CS4 investigate almost similar routes (Innsbruck - Liezen for CS3 and Innsbruck - Graz for CS4) which cover mountainous areas for almost the whole time. These areas include a variety of different environments like broad and narrow valleys as well as smaller alpine passes.

These case studies attempt to represent a broad range of challenging conditions for GNSS encountered in the railway domain all over Austria.

\begin{tabular}{lccc} 
Test case & Date & Route & Processing \\
\hline CS1 & 11.05 .2017 & Salzburg - Vienna airport & CSRS-PPP/PPP-Wizard \\
CS2 & 28.09 .2017 & Salzburg - Klagenfurt & CSRS-PPP/PPP-Wizard/SGAMP \\
CS3 & 29.11 .2019 & Innsbruck - Wörgl - Schwarzach/St.Veit - Liezen & CSRS-PPP/PPP-Wizard \\
CS 4 & 01.12 .2019 & Innsbruck - Wörgl - Schwarzach/St.Veit - Liezen - Graz & CSRS-PPP/PPP-Wizard
\end{tabular}

Table 8.1: Overview of conducted case studies

\subsubsection{PPP processing settings}

This section introduces the most important settings which were applied in PPP processing for the test cases introduced in the last section. Table 8.2 presents the main PPP settings used and possible differences between the processing schemes.

GNSS data for processing were provided by ÖBB in from of raw observations available every second in binary format. These were converted to RINEX 2.11 format (Gurtner and Estey 2007) and fed to the PPP processing engines (see Sections 6.5, 6.6, 6.7 and 6.8). 3D-coordinates, receiver clock error and ZWD were estimated every second in PPP processing.

All processing schemes outlined in Chapter 6 were applied at least for one test case (an overview is given in Table 8.1). In the following, these schemes are referred to as:

- CSRS-PPP: CSRS-PPP

- GAMP using SEID model (as detailed in Section 6.7): SGAMP 
- PPP-Wizard: PPPW

- PPP-Wizard with height-constraint (implemented as detailed in Section 6.8): PPPW-HC

Depending on the actual processing scheme either SF- or DF-PPP for GPS and GLONASS observations were used. IGS final products were utilized for satellite orbit and clock corrections.

lonospheric corrections are applied for CSRS-PPP through IGS GIM broadcast TEC models and for PPPW by obtaining EGNOS (European Geostationary Navigation Overlay Service) corrections. Since the SGAMP scheme operates in DF-PPP mode, the IF-LC is used here.

The hydrostatic part of the tropsopheric delay (i.e. ZHD) is modelled using VMF1 grids for CSRS-PPP, with the simple Saastamoinen model (Equation 4.22) for PPPW and using the Global Mapping Function (GMF, Boehm et al. (2006)) for SGAMP.

For the a-priori constraint settings of the Kalman Filter $\left(\sigma_{L}, \sigma_{P}, \sigma_{Z W D}, \sigma_{\text {Position }}\right)$ a range of values is given since a number of different setups were tried out and the optimal settings vary between the test tracks.

Both code and phase sigma $\left(\sigma_{P}, \sigma_{L}\right)$ were set to significantly higher values compared to their typically magnitude (see Chapter 2, Section 2.3.3). This accounts for the rather low quality of the raw GNSS data and the difficult environmental conditions (e.g. multipath) they were gathered in.

ZWD $\left(\sigma_{Z W D}\right)$ was set in the range from $5 \mathrm{~mm}$ to $1 \mathrm{~cm}$ depending on the test case. Many different settings were tried out for this parameter and the presented ones were found to provide the best results in the ZTD validation. The chosen values are typically of quite low magnitude, but showed reasonable results for all test cases. Possible reason for the good fit of this choice could be the dry weather conditions for the test dates (unfortunately for the assimilation studies, see also Section 8.7). Furthermore for almost all test cases, the trains spent a significant amount of time at fairly high altitude where the influence of water vapour is decreasing, especially in stable and dry weather situations. $7.5^{\circ}$ was chosen as the default setting for the cut-off observation angle. Measurements from elevation angles below this value were not considered in the processing. However, Section 8.6 investigates chances in this as well as other processing settings and their influence on the ZTD solution performance. 


\begin{tabular}{lc} 
Parameter & Setting \\
\hline Mode & Kinematic SF-PPP (CSRS-PPP/PPPW) / DF-PPP (SGAMP) \\
GNSS & GPS + GLONASS \\
Phase Ambiguities & Float \\
Observation weighting & Elevation-dependent \\
Orbits & IGS final \\
Clocks & IGS final \\
lonosphere & GIM broadcast (CSRS-PPP)/EGNOS (PPPW)/SEID (SGAMP) \\
ZHD & VMF1 (CSRS-PPP)/Saastamoinen (PPPW)/GMF (SGAMP) \\
Elevation cut-off & $7.5^{\circ}$ \\
$\sigma_{L}$ & $0.1-0.5 \mathrm{~m}$ \\
$\sigma_{P}$ & $1-10 \mathrm{~m}$ \\
$\sigma_{Z W D}$ & $0.005-0.01 \mathrm{~m}$ \\
$\sigma_{P o s i t i o n}$ & $10-50 \mathrm{~m}$
\end{tabular}

Table 8.2: General PPP settings chosen for the test cases. 


\subsection{Case study 1: 11.05 .2017}

The first test case (CS1) is based on tracking data from a railjet train travelling from Salzburg to Vienna airport on 11.05.2017. The travelling route, obtained from the CSRS-PPP coordinate solution, is shown in Figure 8.1. This first test track covers relatively moderate height (i.e. ZTD) differences with a steady decrease (increase) in height (ZTD) towards Vienna as indicated in Figures 8.4 and 8.5 in the following section. Nevertheless frequent encountering of urban areas and tunnels still produce challenging conditions of GNSS processing.

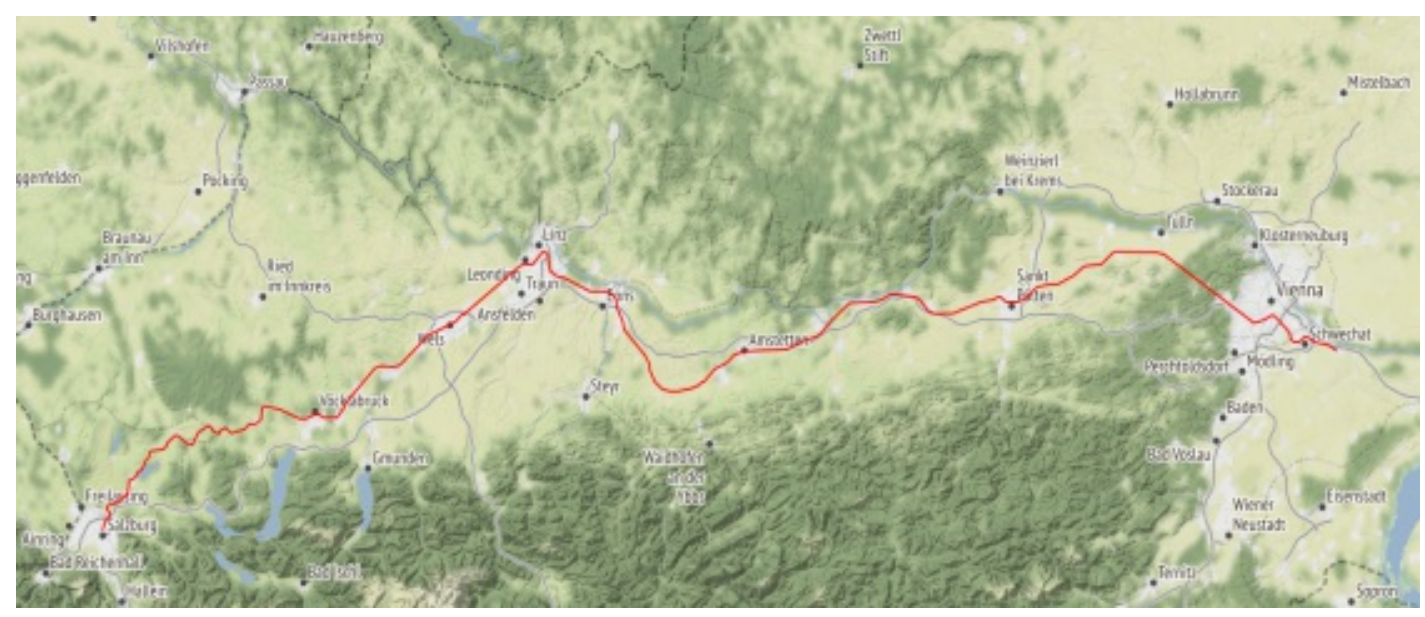

Figure 8.1: Train-track covered for CS1 (Salzburg - Vienna airport) derived from CSRS-PPP processing.

\subsubsection{Raw data quality}

At first, some quality indicators of the data in form of multipath analysis and geometry parameters are shown in Figures 8.2 and 8.3. Figure 8.2 shows the computed CMC observables along the CS1 route. Critical sections, which are encountered while going through urban areas in the beginning (Salzburg) and towards the end of the time series (Vienna), are visible. These sections also exhibit a clearly increased parameter noise in the PPP solution, as shown in Figures 8.4 and 8.5 later.

Furthermore, Figure 8.3 shows the number of observed satellites and computed GDOP along the track. The observed decreases in satellite availability and increases in GDOP values match well with the increased parameter noise in ZTD and height coordinate at the same time (Figures 8.4 and 8.5) as well as increased multipath influence (Figure 8.2). In general, high variability is observed for both satellite visibilty and GDOP, which indicates the changing and challenging environments the GNSS receiver has to cope 


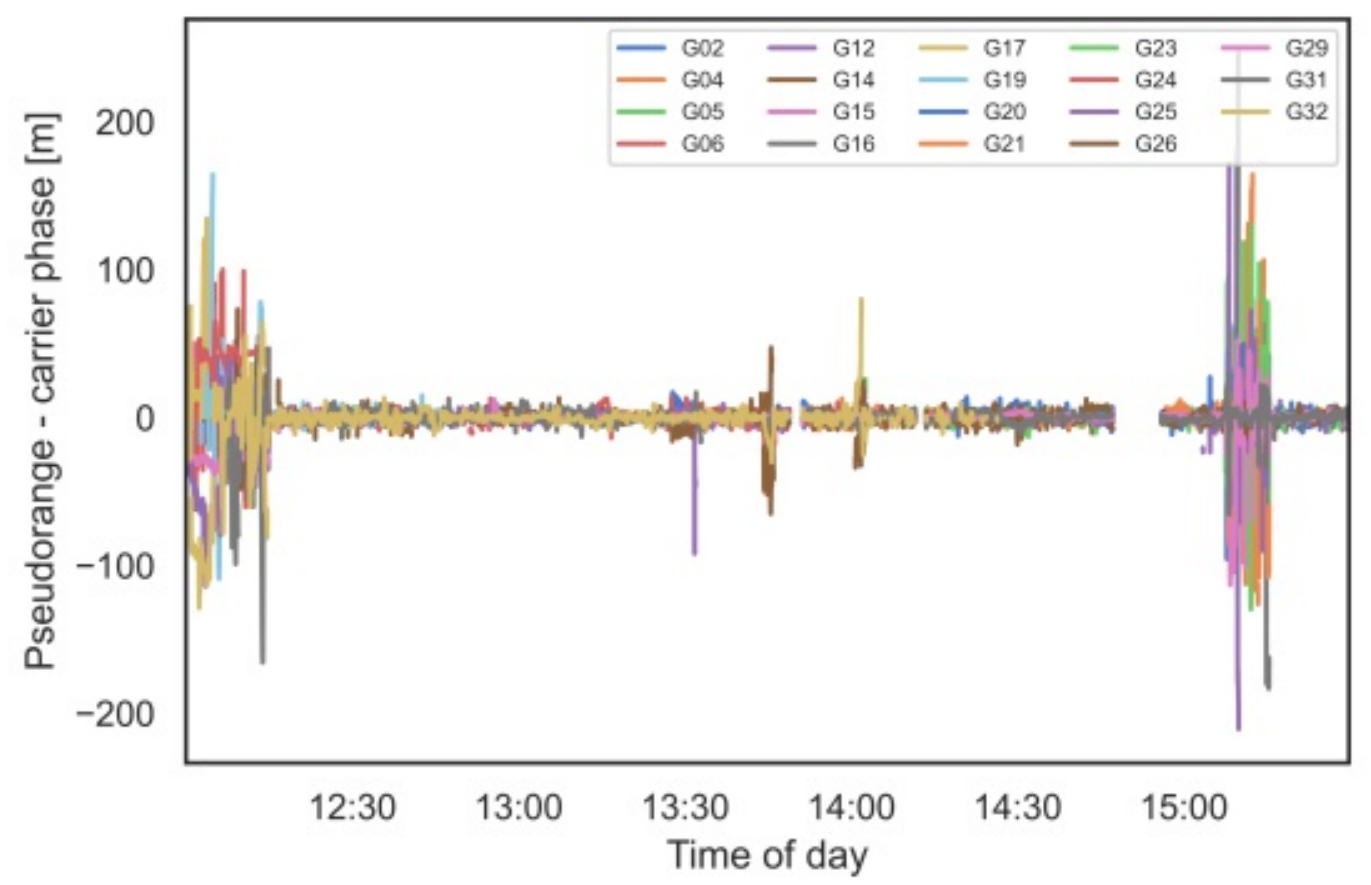

Figure 8.2: Code-Minus-Carrier (CMC) observables for CS1, shown are all visible GPS satellites.

with.
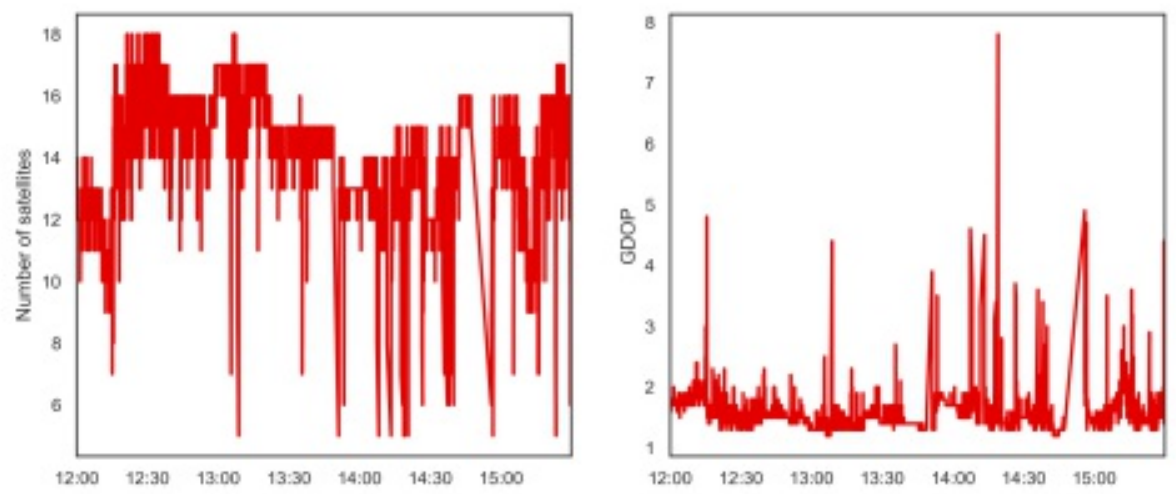

Figure 8.3: Number of visible satellites (left) and GDOP values (right) along the CS1 route.

\subsubsection{PPP noise analysis}

As outlined in the introduction of this chapter, an analysis of the observed noise level for height and ZTD time series of CSRS-PPP and PPPW is carried out here. Therefore a smoothed solution was produced using a MA-filter with a time window of five minutes 
as detailed in Section 8.1. Figures 8.4 and 8.5 show a comparison of processing results for ZTD and height coordinate respectively, derived from CSRS-PPP (top) and PPPW (bottom). The actual output of the respective PPP engine (red) and the smoothed time series (blue) are shown for both components.
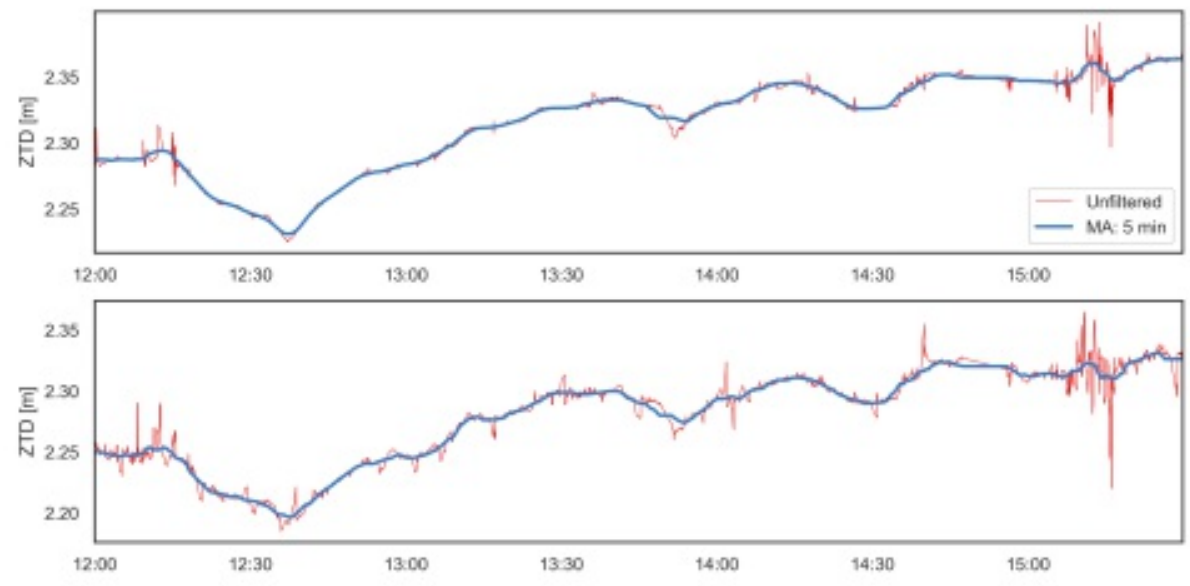

Figure 8.4: CS1 PPP ZTD time series from CSRS-PPP (top) and PPPW (bottom). The raw PPP ZTD estimates are shown in red, the MA-smoothed (Interval $5 \mathrm{~min}$ ) in blue.
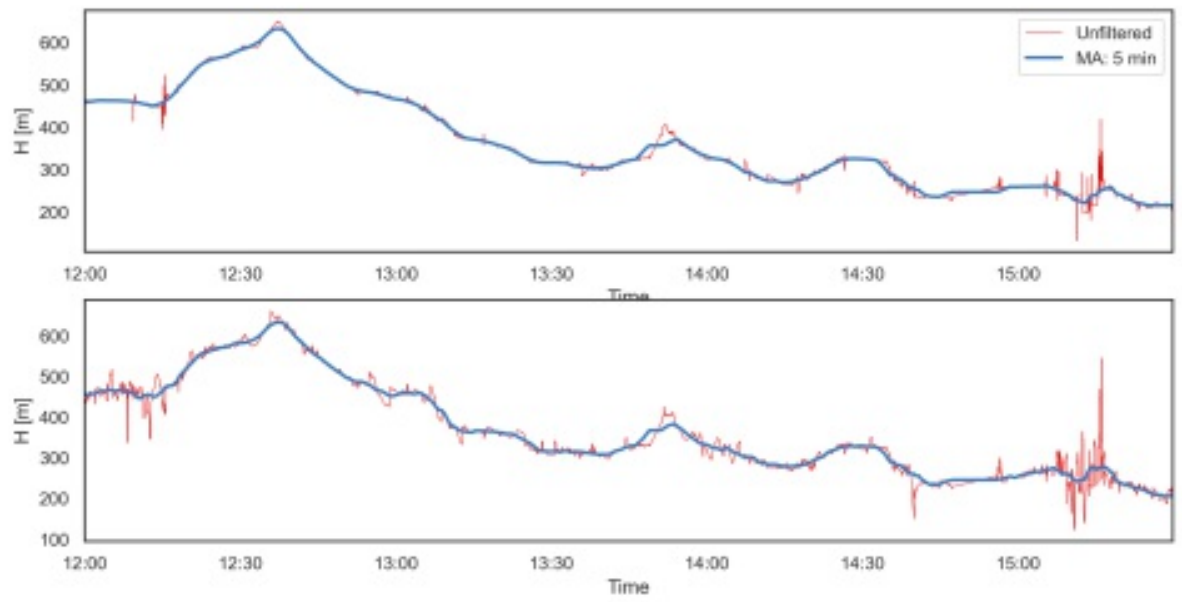

Figure 8.5: CS1 PPP ellipsoidal height time series from CSRS-PPP (top) and PPPW (bottom). The raw PPP height estimates are shown in red, the MA-smoothed (Interval $5 \mathrm{~min}$ ) in blue.

The comparison allows a quantification of the observed parameter noise through standard deviation and RMS, as indicated by the magnitude of the red spikes which are visible in 
the plots.

The noise level in ZTD varies by a factor of $\sim 7-10$ between CSRS-PPP and PPPW, with CSRS-PPP showing magnitudes at the millimetre and PPPW at the centimetre level for RMS and standard deviation. Bias values are vanishingly small for ZTD $\left(10^{-3}\right.$ $-10^{-4} \mathrm{~cm}$ ), which is expected from the comparison between a time series and its MAsmoothed version. This fact therefore suggests that the time window of five minutes for the MA-filter is an appropriate choice to filter noise from these time series.

For the height coordinate, the noise level varies by a factor of $\sim 2$ (for both RMS and standard deviation) between the respective schemes. Standard deviation and RMS are below 10 metres for CSRS-PPP (8.769/9.289) and between 15 - 20 metres for PPPW (17.586/19.690). In terms of median a clear advantage of CSRS-PPP is evident, for both ZTD and height coordinate.

All performance measures concerning the noise level of both solutions are summarized in Table 8.3 and 8.4 .

\begin{tabular}{lcccc} 
Processing scheme & Bias [cm] & Standard deviation $[\mathbf{c m}]$ & RMS [cm] & Median $[\mathbf{c m}]$ \\
\hline CSRS-PPP & 0.0004 & 0.3 & 0.3 & 0.03 \\
PPP-Wizard & -0.001 & 2.0 & 2.3 & 0.39
\end{tabular}

Table 8.3: Performance metrics for the PPP noise analysis of CS1 for CSRS-PPP and PPPW. Measures are computed for ZTD differences between original PPP output and smoothed solution.

\begin{tabular}{lcccc} 
Processing scheme & Bias [m] & Standard deviation [m] & RMS [m] & Median [m] \\
\hline CSRS-PPP & -0.017 & 8.769 & 9.289 & 0.645 \\
PPP-Wizard & 0.011 & 17.586 & 19.690 & 4.240
\end{tabular}

Table 8.4: Performance metrics for the PPP noise analysis of CS1 for CSRS-PPP and PPPW. Measures are computed for height coordinate differences between original PPP output and smoothed solution.

\subsubsection{ZTD validation}

For the validation of the ZTD estimates derived from CSRS-PPP and PPPW, reference ZTD values have been calculated from ERA5 reanalysis data for each case study, using the methodology detailed in Chapter 7. Results for CS1 are shown in Figure 8.6. A similar pattern for both GNSS- and ERA5-ZTD can be seen for the latter part of the time series. However, for the first hour (12-13 UTC), GNSS- and ERA5-derived 
ZTD time series deviate significantly from each other. While ERA5-ZTD shows a maximum for this period, the almost exact opposite behaviour (minimum) is found in the GNSS-ZTD solutions. There are two possible explanations for this: (1): very localized occurrence of high water vapour amount not captured by GNSS or (2): errors/artefacts in the corresponding ERA5 fields. The latter might be more likely, considering the dry weather conditions observed on this day and the fact that both GNSS-ZTD solutions are in good agreement with each other (similar patterns and thus high correlation between them). Nevertheless it is interesting to notice that in the corresponding area along the track multiple lakes of varying size are located (see Figure 8.1). This may lead to very localized, high amounts of water vapour as well as possible artefacts in modelled humidity fields in the ERA5 reanalysis data. However further research would be needed to back up one of those possible explanations.

Comparing the performance of the ZTD estimation between the two different software packages used, a diverse picture can be drawn for this test case. In the first part of the track (first hour approximately), the CSRS-PPP ZTD solution outperforms the PPPW solution in terms of deviations from the ERA5-ZTD reference. However, this part represents the time where the significant deviations in the signal patterns between GNSS and ERA5 are observed and therefore deviations might be interpreted with caution. For the latter (and time-wise much larger) part, which shows highly correlated patterns between GNSS and ERA5, PPPW results are closer to ERA5. This fact is also represented in lower bias (by factor of $\sim 3$ ) and RMS values (factor of $\sim 1.5$ ) compared to CSRS-PPP as indicated in Table 8.5. Standard deviations of ZTD differences to ERA5 are comparable for both schemes (around $4 \mathrm{~cm}$, see also Table 8.5).

Figure 8.7 shows the distributions of ZTD differences to ERA5 for both processing schemes. Clearly visible is the fairly large number of strongly positive values for both schemes. This is also reflected in the (strictly positive) bias values, especially for CSRS-PPP which has no larger negative deviations to ERA5 like PPPW (see x-axis of Figure 8.7).

The computed correlation coefficients and linear fits between GNSS- and ERA5-ZTD solutions are heavily influenced by the large deviations observed in the first hour of this test case. Both GNSS-ZTD solutions show almost no, to slightly negative correlation with ERA-ZTD, as shown in Figure 8.8. Since both GNSS-ZTD solutions agree very well with each other and this part of the track does not denote particularly challenging environments for GNSS, the more likely explanation are problems in the ERA5 dataset as already discussed above. 

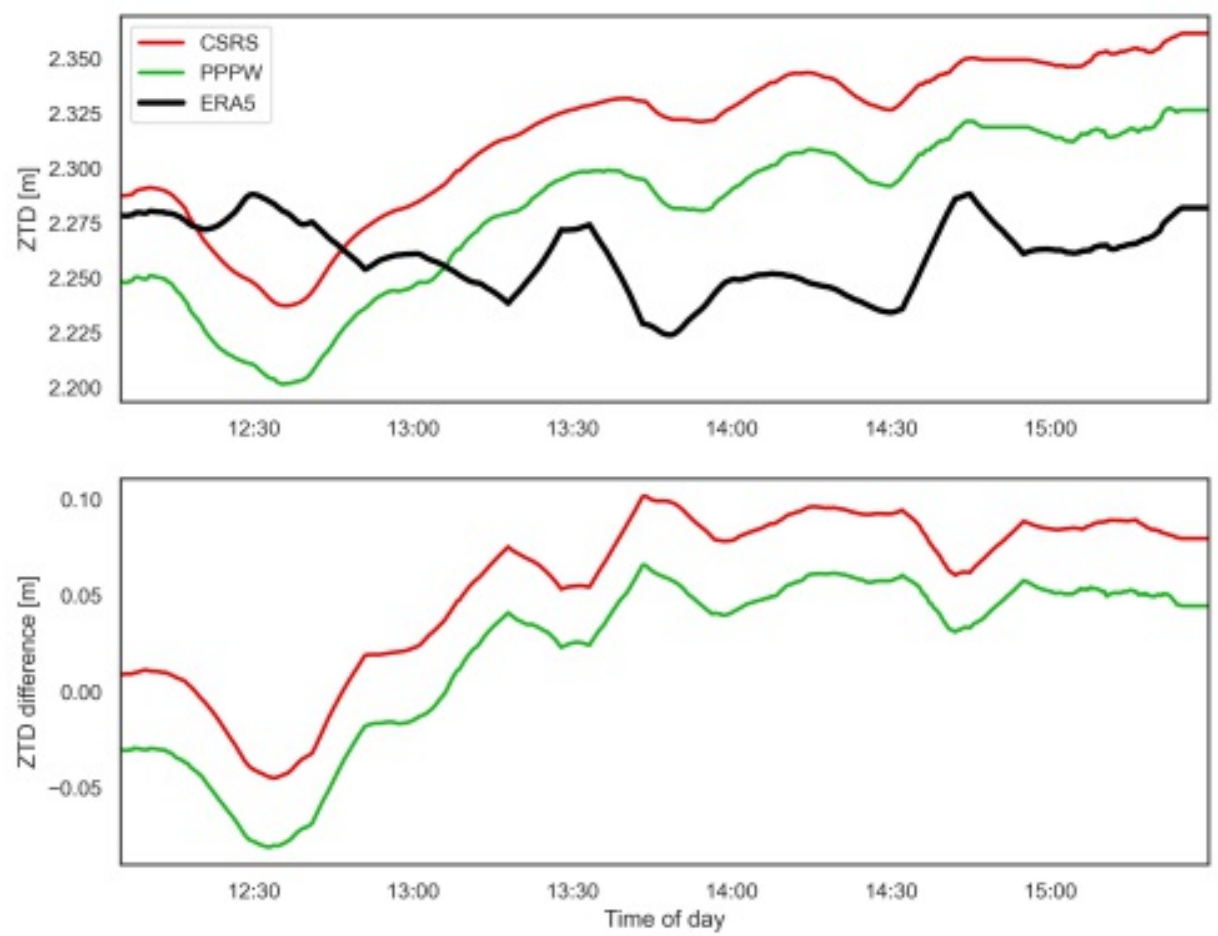

Figure 8.6: Comparison between estimated GNSS-ZTD (CSRS-PPP (red) and PPPW (green)) and ERA5-ZTD (black) for CS1 (11.05.2017), Top: absolute values, bottom: ZTD differences between GNSS-ZTD with respect to ERA5-ZTD
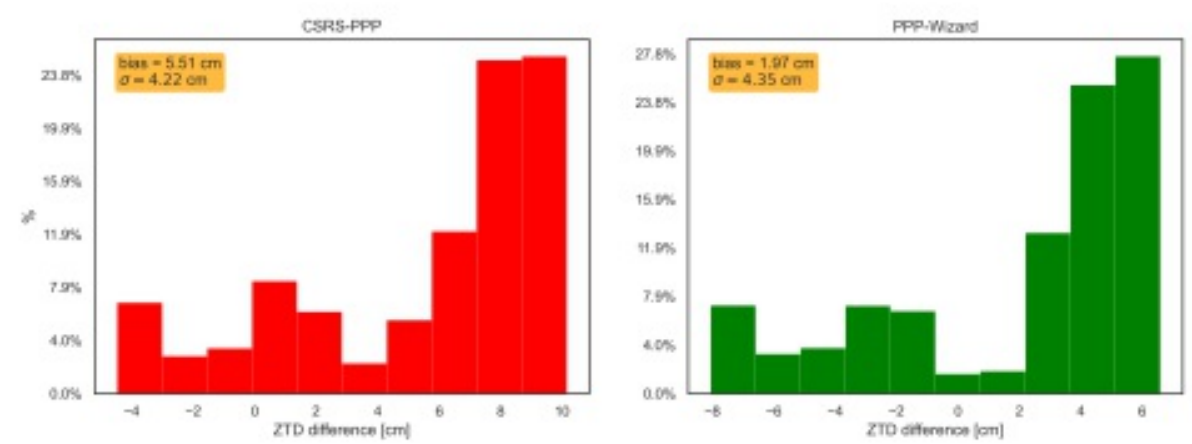

Figure 8.7: Histograms of ZTD differences between GNSS-ZTD and ERA5-ZTD for test case CS1. Left: CSRS, Right: PPPW. Also indicated are bias and standard deviation $(\sigma)$ of the respective solutions.

The influence of the deviations between GNSS-ZTD and ERA5-ZTD becomes even more evident when comparing Figure 8.8 to Figure 8.9 , where the latter shows the correlation between the respective time series when excluding the problematic part in 

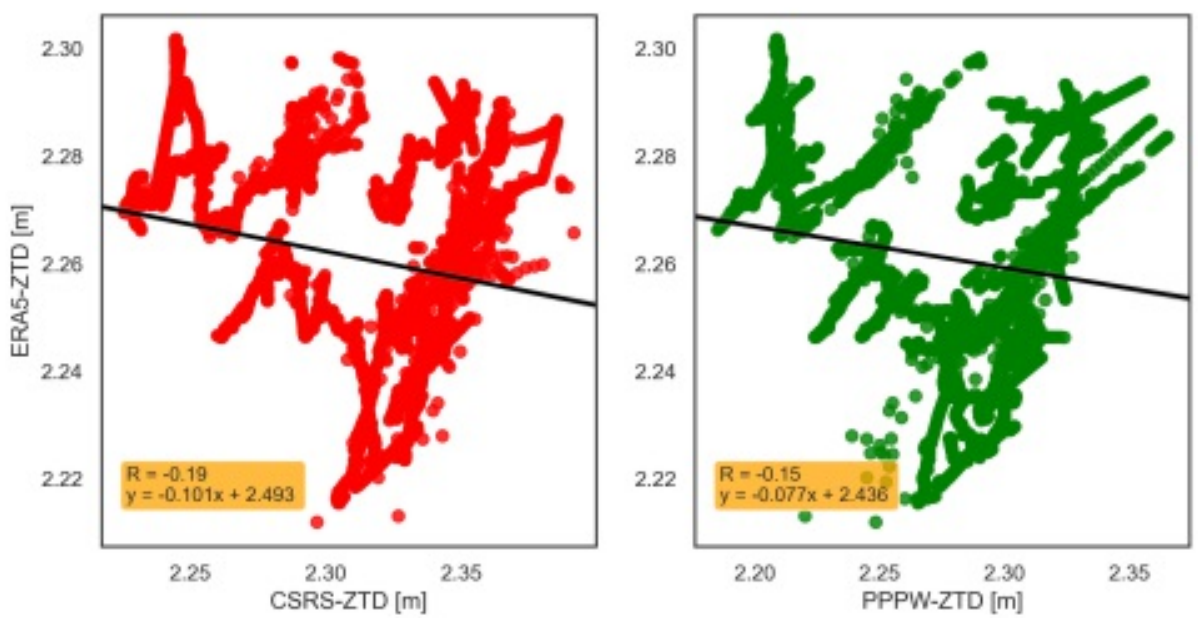

Figure 8.8: Correlation between ZTD as estimated by each software package and the ERA5 reference values for CS1, 11.05.2017: (left) CSRS-PPP and (right) PPPW. Terms R and y represent the correlation coefficient and the linear fit, respectively.

the beginning (12:00 - 13:30). The correlation coefficients are significantly improved and close to the high levels which are present for the other test cases. On the other hand, the statistics of this shortened time series (Table 8.6) show increased bias and RMS values. This is caused by the fact that the significant positive biases of both GNSS-ZTD time series are not compensated by the negative biases present in the beginning of the complete test data. A complete overview of statistical performance metrics for the ZTD validation can be found in Table 8.5 (full time series) and 8.6 (excluding 12:00 -13:30).

Apart from these deviations in the first hour, results from CS1 in general are promising since the patterns of GNSS- and ERA5-ZTD agree well for the remaining part. Nevertheless a non-negligible bias in centimetre-level is observed between GNSS- and ERA5-ZTD time series.

\begin{tabular}{lcccc} 
Processing scheme & Bias [cm] & Standard deviation [cm] & RMS [cm] & Correlation \\
\hline CSRS-PPP & 5.51 & 4.22 & 6.85 & -0.19 \\
PPP-Wizard & 1.97 & 4.35 & 4.72 & -0.15
\end{tabular}

Table 8.5: CS1: Performance metrics for the ZTD validation against ERA-ZTD for processing results of CSRS-PPP and PPPW. 

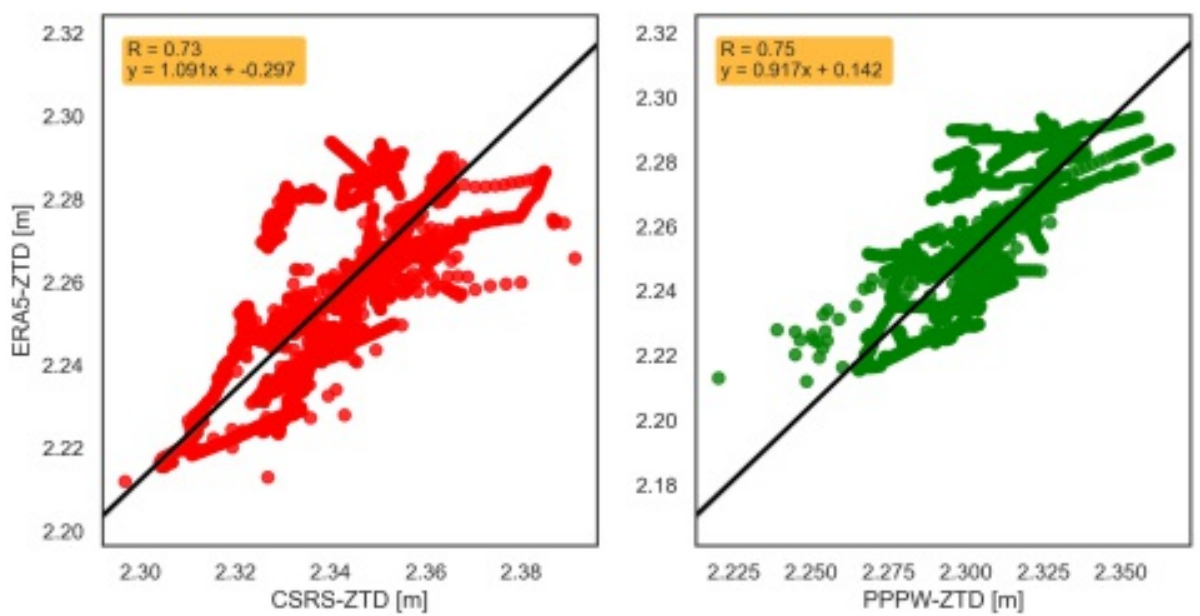

Figure 8.9: Correlation between ZTD as estimated by each software package and the ERA5 reference values for CS1, excluding the first hour of data (12:00-13:30): (left) CSRS-PPP and (right) PPPW . Terms $\mathrm{R}$ and y represent the correlation coefficient and the linear fit, respectively.

\begin{tabular}{lcccc} 
Processing scheme & Bias [cm] & Standard deviation [cm] & RMS [cm] & Correlation \\
\hline CSRS-PPP & 8.39 & 1.07 & 8.46 & 0.73 \\
PPP-Wizard & 4.95 & 0.91 & 5.03 & 0.75
\end{tabular}

Table 8.6: CS1 (excluding 12:30 -13:30): Performance metrics for the ZTD validation against ERA-ZTD for processing results of CSRS-PPP and PPPW. 


\subsection{Case study 2: 28.09 .2017}

CS2 uses data collected by a Railjet train on 28.09.2017, the travelling track (coordinate solution from CSRS-PPP) is shown in Figure 8.10. The train started in Munich (around 06 UTC) and arrived in Salzburg around 08 UTC. From there it passed the alpine ridge southwards towards Klagenfurt, where it terminated shortly before 12 UTC. Especially

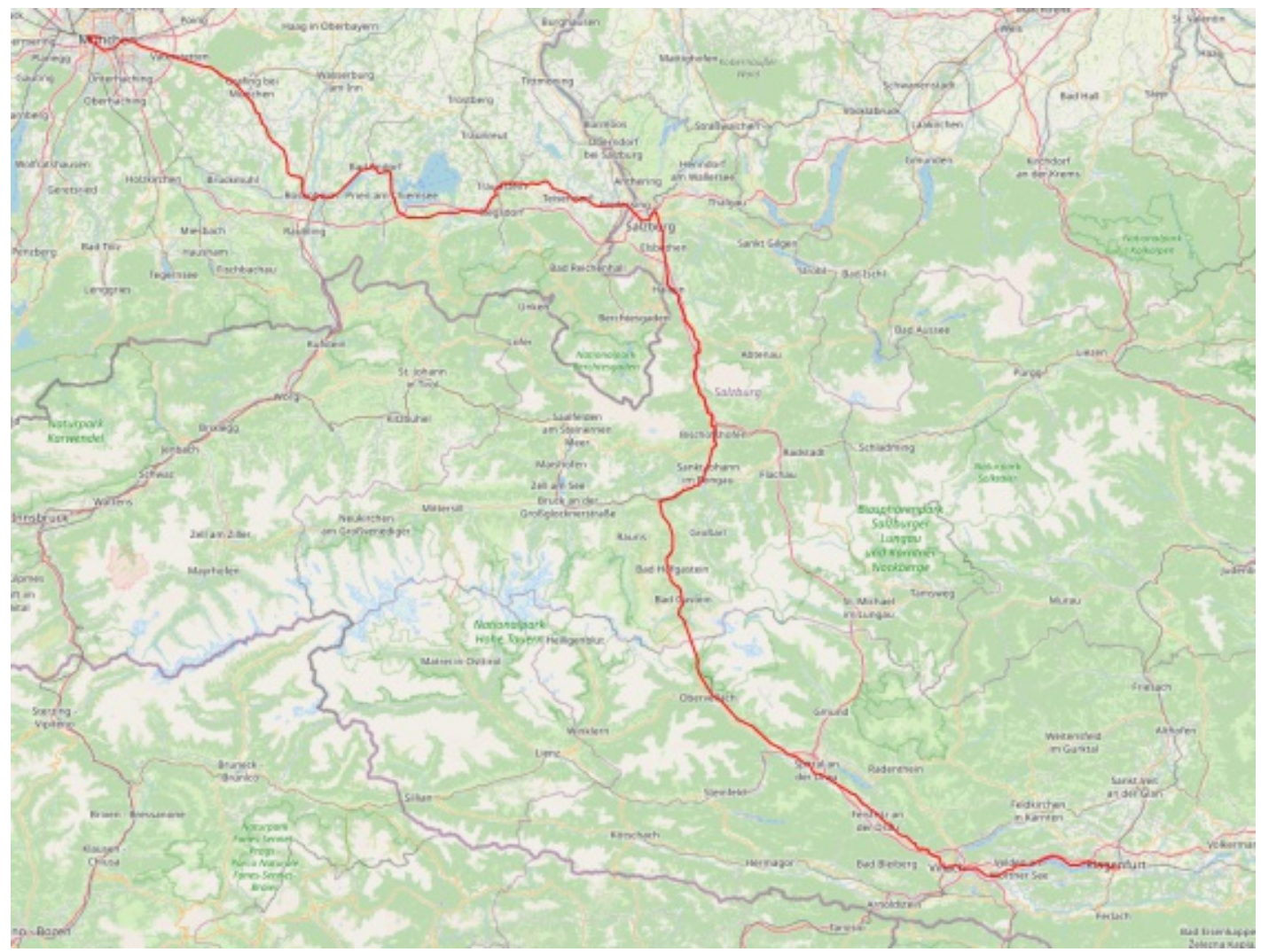

Figure 8.10: Train-track covered for CS2 (Munich - Klagenfurt shown here) derived from CSRS-PPP processing.

the passing of the alpine ridge (where heights over 1000 meters are reached) makes this a particularly interesting test case. Therefore, the period covering the alpine passing (Salzburg-Klagenfurt), which corresponds to 08-12 UTC, was selected for detailed analysis of the results.

\subsubsection{Raw data quality}

Discussion of the results again starts with an overview on multipath and geometry indicators. Figure 8.11 shows the CMC observables for CS2 for all visible GPS satellites. As for CS1, high multipath influence is experienced especially within parts where urban environments are encountered (in the beginning Salzburg, at the end Villach and Kla- 
genfurt). Furthermore smaller tunnels in the middle of the track can also be recognized, as no solution is available there.

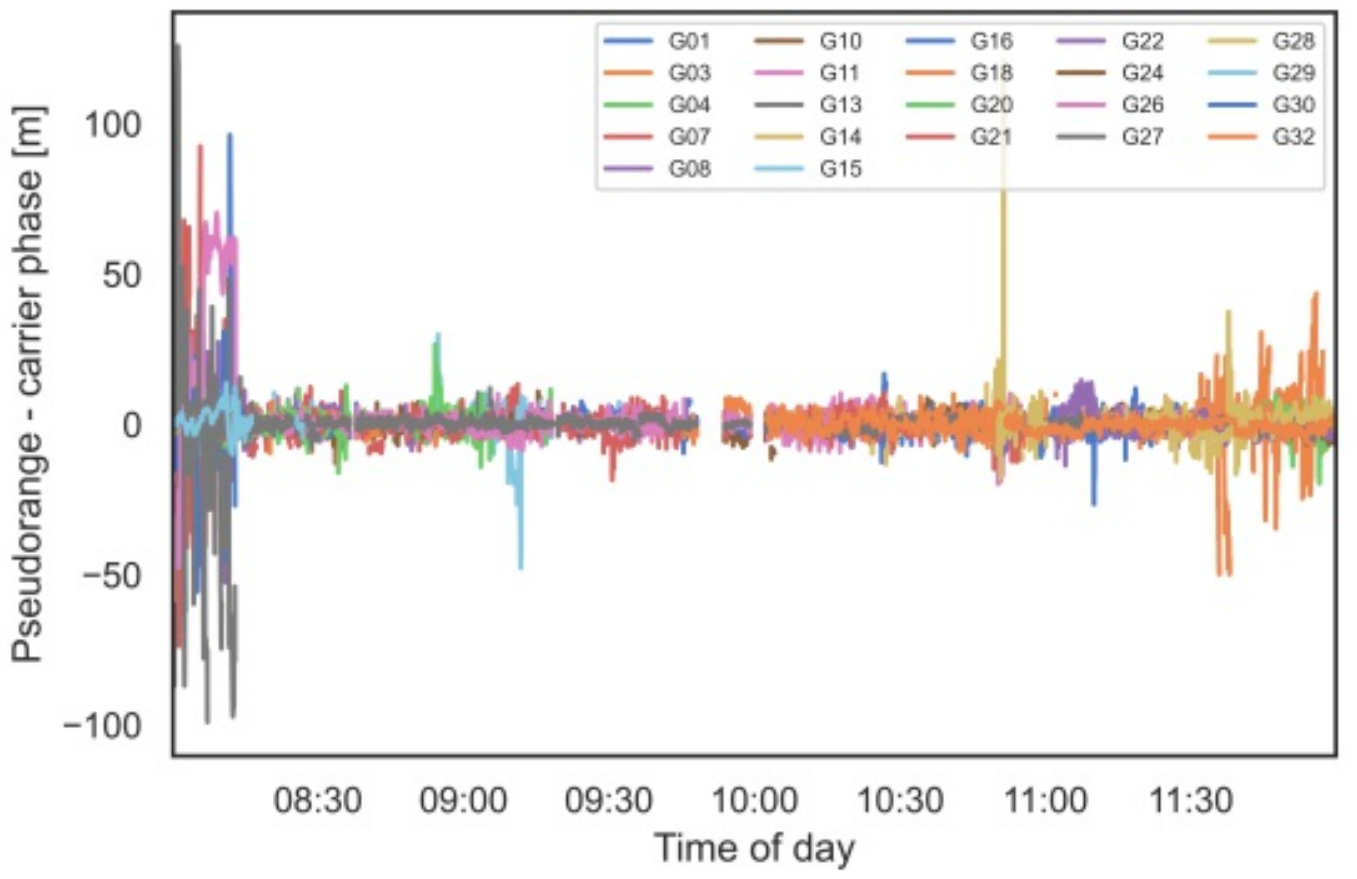

Figure 8.11: CMC observables for CS2, shown are all visible GPS satellites.

A quite coherent picture to the multipath levels shows Figure 8.12, which presents the number of observed satellites and computed GDOP values along the track. Here substantial spikes (low number of satellites) and a high GDOP indicate sections of distorted geometry and therefore challenging conditions of GNSS operation. These sections match approximately with times of high multipath influence (Figure 8.11) and increased parameter noise (Figures 8.13 and 8.14).

\subsubsection{PPP noise analysis}

Similar analysis of the parameter noise level as already presented for CS1 was carried out for CS2. Again the original PPP output from CSRS-PPP and PPPW was compared against a smoothed (again MA-filtered, again five minute time window) solution for ZTD and height coordinate. The comparisons are shown in Figures 8.13 and 8.14.

Overall the solutions draw a similar picture as those from the first test case. Most statistical measures outlined in Tables 8.7 and 8.8 are comparable, sometimes almost similar to those of CS1. The time series derived using PPPW again show significantly increased noise and standard deviation compared to the CSRS-PP solution, especially in ZTD (by factor 3-4). Nevertheless the performance of the PPPW ZTD solution is 

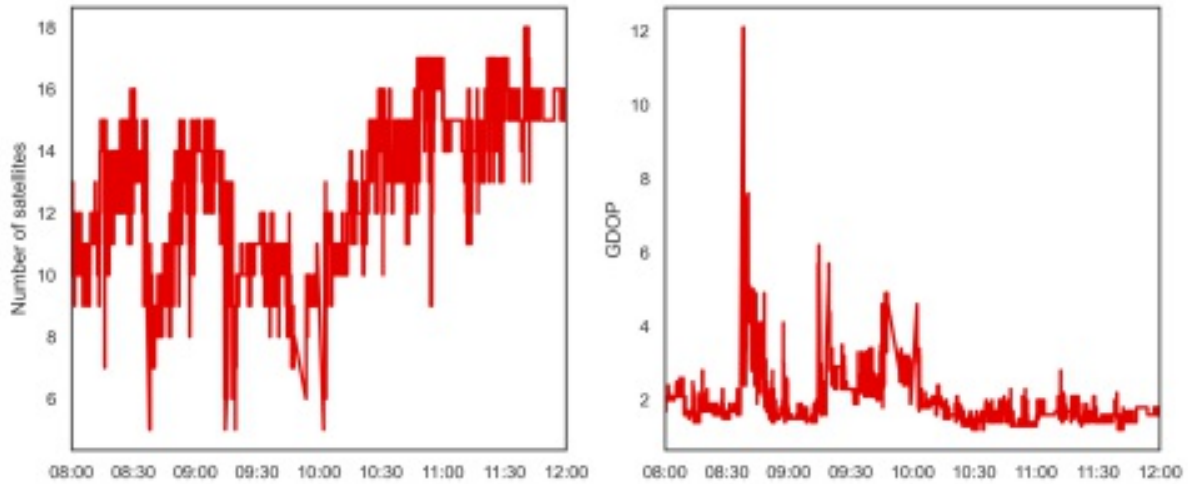

Figure 8.12: Number of visible satellites (left) and GDOP values (right) along the CS2 route.

remarkably increased compared to the results from CS1 (50\% smaller standard deviation and RMS). The noise level of the height coordinate largely remain the same, with RMS and standard deviation in the millimetre-range for CSRS-PPP and in centimetre-range for PPPW. This represents a very promising first indication of reasonable performance of the results, especially for CSRS-PPP.

For the height coordinate, statistics are almost equal to CS1. Again the PPPW time series exhibits a factor 2-3 larger noise compared to CSRS-PPP. This is also evident in the bias values (-0.002/-0.023), differing by a factor 10 between the solutions and indicating decreasing performance of the 5-minute MA-filter for time series with increased noise as for the PPPW solution in this case.

Furthermore, at the highest section of the track very challenging conditions or even GNSS-denied areas in form of tunnels are encountered, which manifests itself in some smaller jumps in the results of both schemes. Nevertheless, a reasonable solution is recovered quite fast for both schemes after these discontinuities.

As already outlined before, the multipath-prone sections (mostly at the beginning of the route) are also visible in terms of increased parameter noise, especially in the PPPW time series.

A detailed summary of statistics can be found in Table 8.7 and 8.8. 

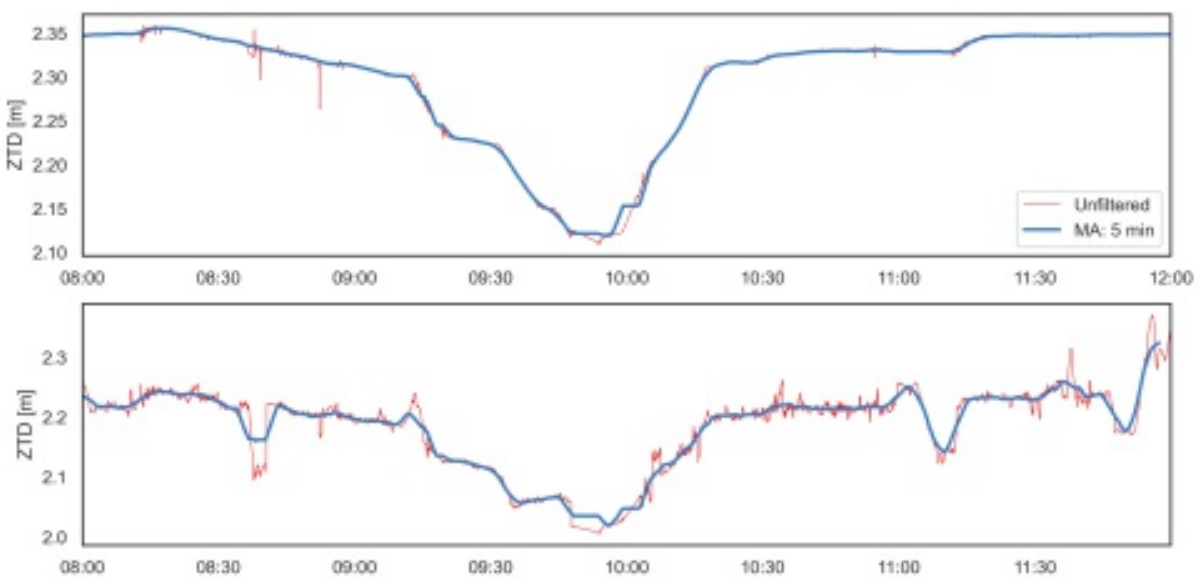

Figure 8.13: CS2 PPP ZTD time series from CSRS-PPP (top) and PPPW (bottom). The raw PPP ZTD estimates are shown in red, the MA-smoothed (Interval $5 \mathrm{~min}$ ) in blue.
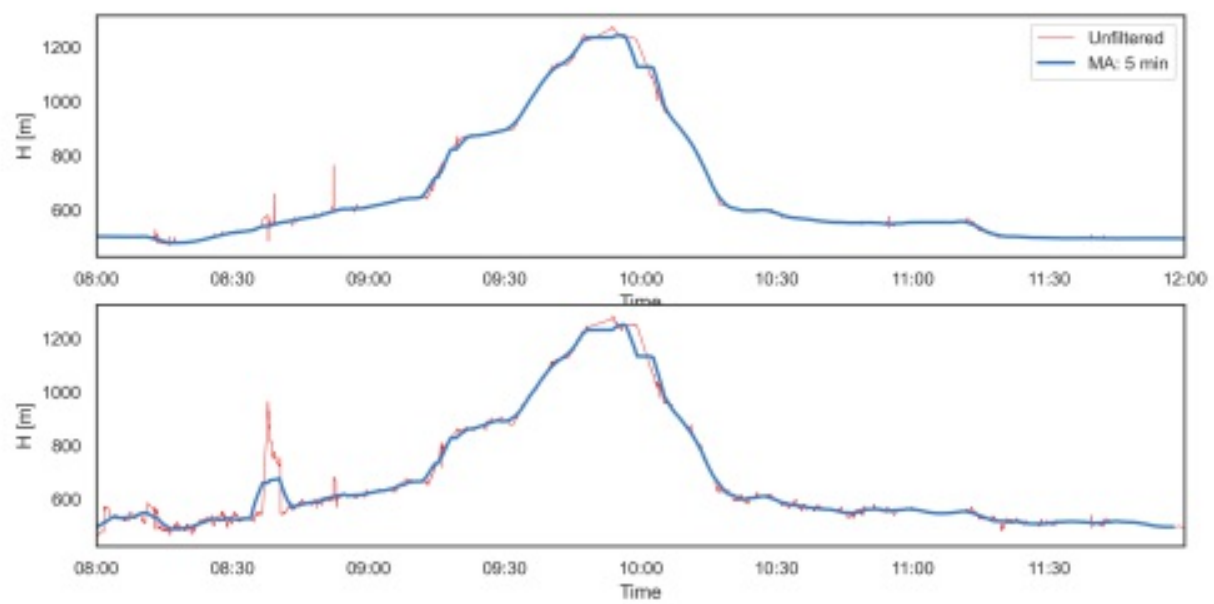

Figure 8.14: CS2 PPP ellipsoidal height time series from CSRS-PPP (top) and PPPW (bottom). The raw PPP height estimates are shown in red, the MA-smoothed (Interval 5 min) in blue. 


\begin{tabular}{lcccc} 
Processing scheme & Bias $[\mathrm{cm}]$ & Standard deviation $[\mathrm{cm}]$ & RMS [cm] & Median [cm] \\
\hline CSRS-PPP & 0.004 & 0.3 & 0.3 & -0.001 \\
PPPW & 0.01 & 1.0 & 1.3 & 0.012
\end{tabular}

Table 8.7: Performance metrics for the PPP noise analysis of CS2 for CSRS-PPP and PPPW. Measures are computed for ZTD differences between original PPP output and smoothed solution.

\begin{tabular}{lcccc} 
Parameter & Bias [m] & Standard deviation [m] & RMS [m] & Median [m] \\
\hline CSRS-PPP & -0.002 & 7.269 & 7.801 & 0.004 \\
PPPW & -0.023 & 20.095 & 20.022 & 0.357
\end{tabular}

Table 8.8: Performance metrics for the PPP noise analysis of CS2 for CSRS-PPP and PPPW. Measures are computed for height coordinate differences between original PPP output and smoothed solution.

\subsubsection{SEID processing}

CS2 is the case where the application of the SEID model is tested for ionospheric mitigation. Therefore data from a number of reference stations was utilized within the model. The chosen stations are listed in Table 8.9 and the track map with the stations location indicated is shown in Figure 8.15. As visible from the map and table, seven stations

\begin{tabular}{lc} 
Station & Distance from reference point [km] \\
\hline Gummern & 47.6 \\
Oching Süd & 4.9 \\
Rötenkogel & 55.2 \\
Saalfelden & 66.0 \\
Salzburg & 62.4 \\
Sillian & 66.2 \\
Schladming & 62.4
\end{tabular}

Table 8.9: Reference stations chosen for SEID processing for CS2.

are chosen for the SEID processing. The decision which and how many stations are selected is based on the distance from a defined reference point. This reference point was determined in a static way here as the midpoint (median) of the trajectory. All stations within $80 \mathrm{~km}$ distance are selected. This threshold decision is based on former studies 


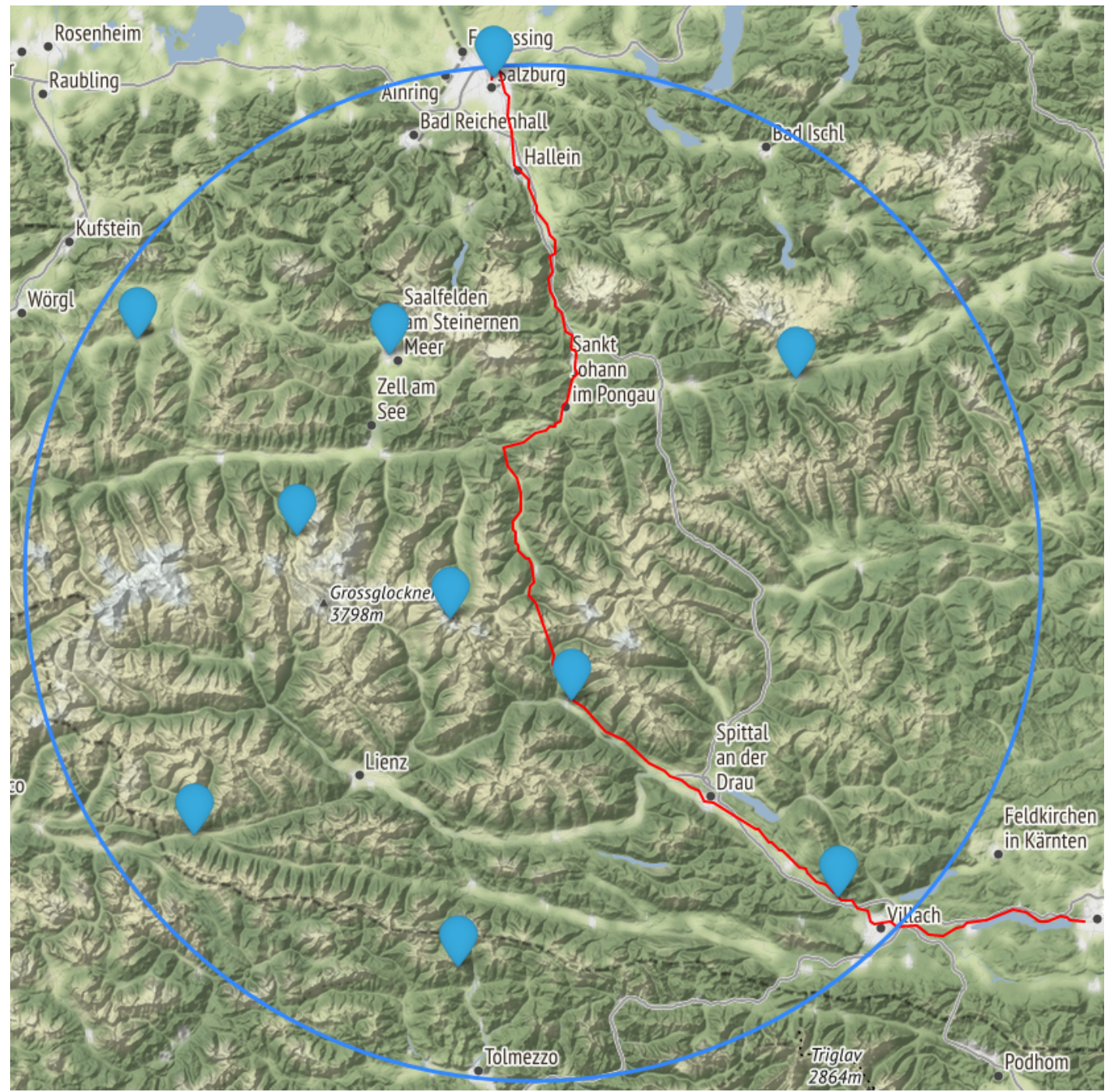

Figure 8.15: Train-track covered for CS2 with the reference stations utilized for SEID processing shown in blue dots.

and clearly variable under different circumstances (e.g. higher/lower number of stations available within shorter distance, i.e. denser/less dense reference station network). This is clearly a simplified approach for a kinematic application and has to be redefined for operational processing to a kind of moving reference point, which is chosen for a suitable time step. A possible choice for this time step would be, e.g. one hour since:

- EPOSA reference station data in RINEX format is provided at this interval

- lonospheric conditions might not change tremendously within this time step

Although the second point might not always be fulfilled, one hour still seems to be an adequate choice considering average conditions at mid-latitudes. Nevertheless, this choice also limits the output interval for ZTD results if the SEID model is used and 
therefore the topic has to be investigated further in detail.

\subsubsection{ZTD validation}

This section provides the results of the ZTD validation for CS2. The validation is carried out in a similar way as for CS1, only with the difference that results from all four processing schemes introduced in Chapter 6 were available and thus are presented here.
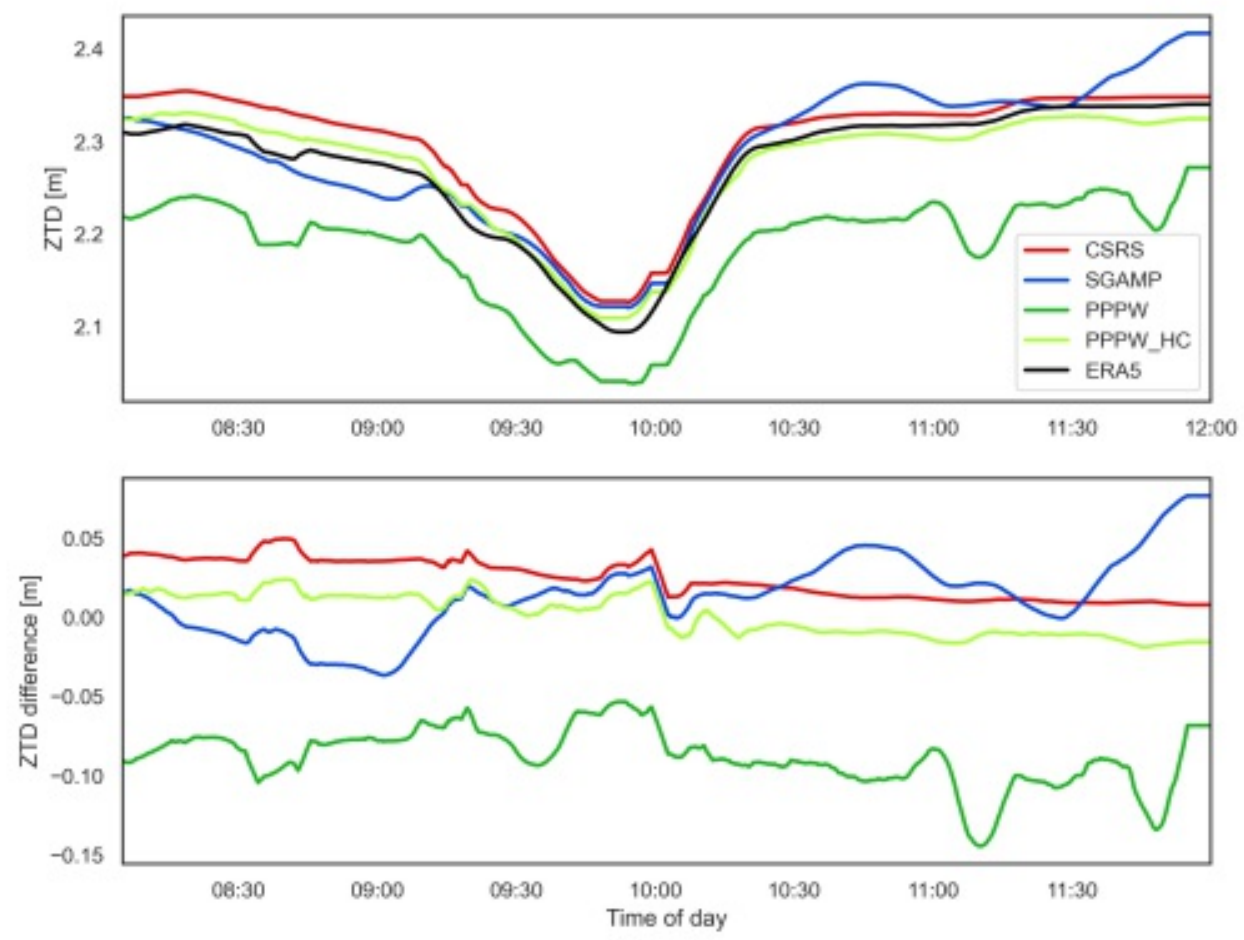

Figure 8.16: Comparison between estimated GNSS-ZTD (CSRS-PPP (red), SGAMP (blue) and PPPW (green) and PPPW-HC (lime)) and ERA5-ZTD (black) for CS2 (28.09.2017), Top: ZTD, bottom: differences between GNSS-ZTD with respect to ERA5-ZTD

Figure 8.16 shows the comparison of GNSS-ZTDs and ERA5-ZTD for the chosen timeframe/route in terms of ZTD values and ZTD differences of the different processing schemes (GNSS-ZTDs) to ERA5-ZTD.

PPPW-HC performs best in the comparison, showing very low bias $(0.1 \mathrm{~cm})$ and RMS $(1.2 \mathrm{~cm})$ values as outlined in Table 8.10. These values are comparable to DF solutions which are commonly used in GNSS Meteorology and therefore represent very promising results from this type of processing. 
SGAMP also shows low values for bias and RMS $(1.3 / 2.8 \mathrm{~cm})$, but has an increased standard deviation (by a factor $\sim 2$ ) compared to the other schemes. Nevertheless these results are able to indicate the potential of the SEID model for SF-GNSS processing and applications like GNSS Meteorology.

CSRS-PPP shows results comparable to SGAMP in terms of bias $(2.4 \mathrm{~cm})$ and similar for RMS $(2.7 \mathrm{~cm})$ and its solution has the lowest standard deviation of all solutions $(1.2$ $\mathrm{cm})$. Compared to the results from CS1, an improvement of $\sim 50 \%$ can be found in terms of bias.

Compared to the results of the other schemes those from PPPW show significantly worse performance in terms of differences to ERA5-ZTD. In the PPPW ZTD time series, a negative offset of about $5-10 \mathrm{~cm}$ is present over the entire analysed period. This is also visible in the distributions of ZTD differences, shown in the respective histograms in Figure 8.17. The pronounced advantage of the height-constrained PPPW (PPPW-HC) over the standard version might indicate a height offset introduced in PPPW which can be erased by applying a height-constraint as described in Section 6.8.

A detailed summary of performance metrics for the ZTD validation are given in Table 8.10.

Overall, the results from the CS2 ZTD validation outline the potential of the newly introduced processing schemes (SGAMP and PPPW-HC), which both are able to outperform the "standard" PPP solutions from CSRS-PPP and PPPW. Especially the good performance of PPPW-HC encourages its usage for the following test cases (CS3 and CS4) as well as for future studies.

\begin{tabular}{lcccc} 
Processing scheme & Bias [cm] & Standard deviation [cm] & RMS [cm] & Correlation \\
\hline CSRS-PPP & 2.4 & 1.2 & 2.7 & 0.99 \\
SGAMP & 1.3 & 2.5 & 2.8 & 0.95 \\
PPPW & -8.8 & 1.8 & 8.9 & 0.97 \\
PPPW-HC & 0.1 & 1.3 & 1.3 & 0.99
\end{tabular}

Table 8.10: CS2: Performance metrics for the ZTD validation against ERA-ZTD for processing results of CSRS-PPP, SGAMP and PPPW and PPPW-HC. 

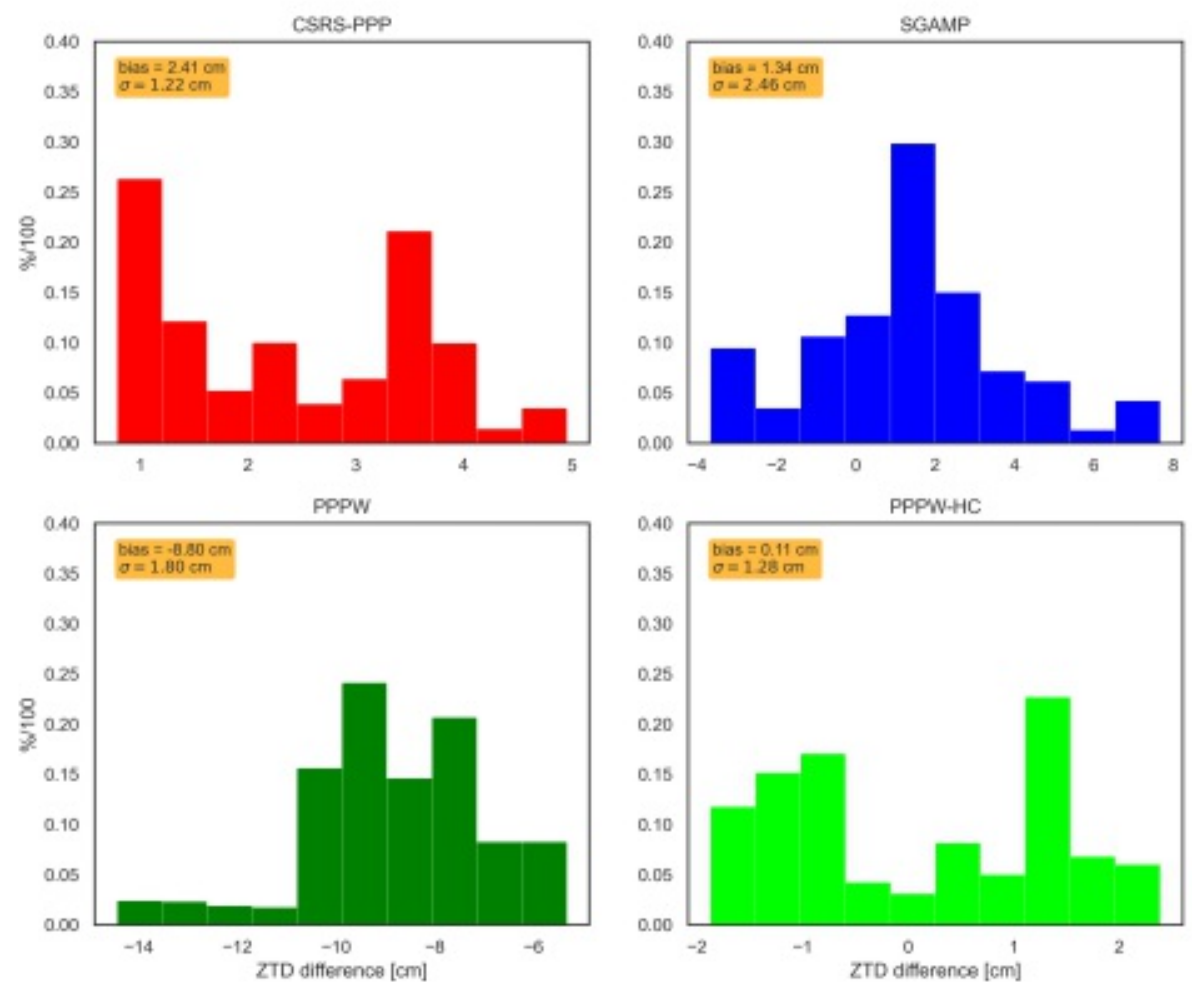

Figure 8.17: Histograms of ZTD differences between GNSS-ZTD and ERA5-ZTD for test case CS2. Top left: CSRS-PPP, Top right: SGAMP, Bottom left: PPPW, Bottom right: PPPW-HC. Also indicated are bias and standard deviation $(\sigma)$ of the respective solutions. 

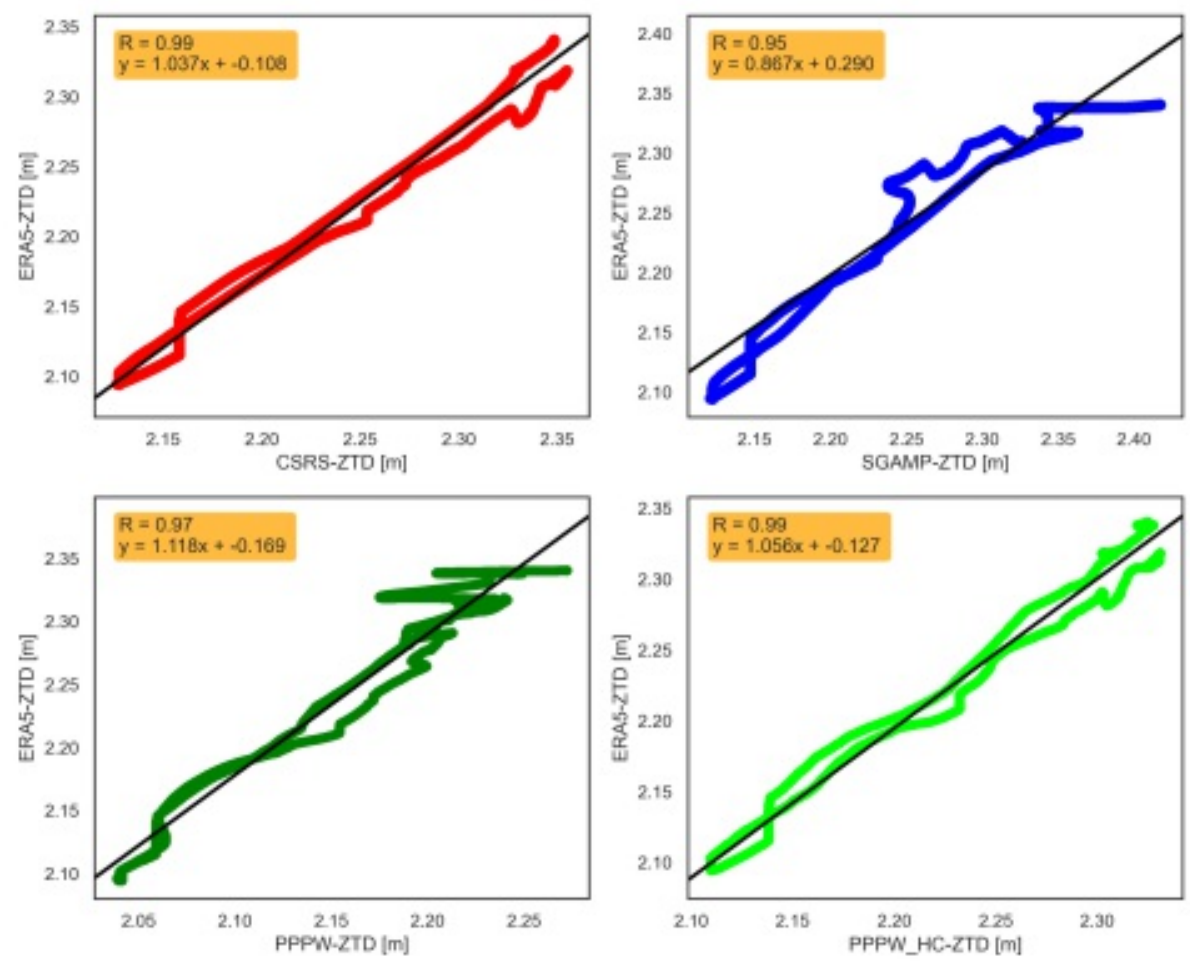

Figure 8.18: Correlation between ZTD as estimated by each software package and the ERA5 reference values for CS2, 28.09.2017. Top left: CSRS-PPP, Top right: SGAMP, Bottom left: PPPW, Bottom right: PPPW-HC. Terms R and y represent the correlation coefficient and the linear fit, respectively. 


\subsection{Case study 3: 29.11.2019}

CS3 uses data gathered from a railjet train on 29.11.2019. This express train was travelling on the route from Innsbruck down the Inn valley to Wörgl, from there via Kitzbühel over to Salzburg and finally terminating in Liezen, Styria.

This route provides very diverse conditions along the track with some prominent features like major cities (Innsbruck, Graz (only for CS4 then)), major (Inn Valley, Enns Valley) and very narrow alpine valleys (Brixen Valley, i.e. Wörgl-Kitzbühel) as well as mountain passes (Grießen pass, Mandling pass). Therefore environmental conditions (multipath, shading, atmospheric conditions) are very different along different sections of the route which is also reflected in the results. The exact travelling track, as derived from CSRSPPP, is visualized in Figure 8.19.

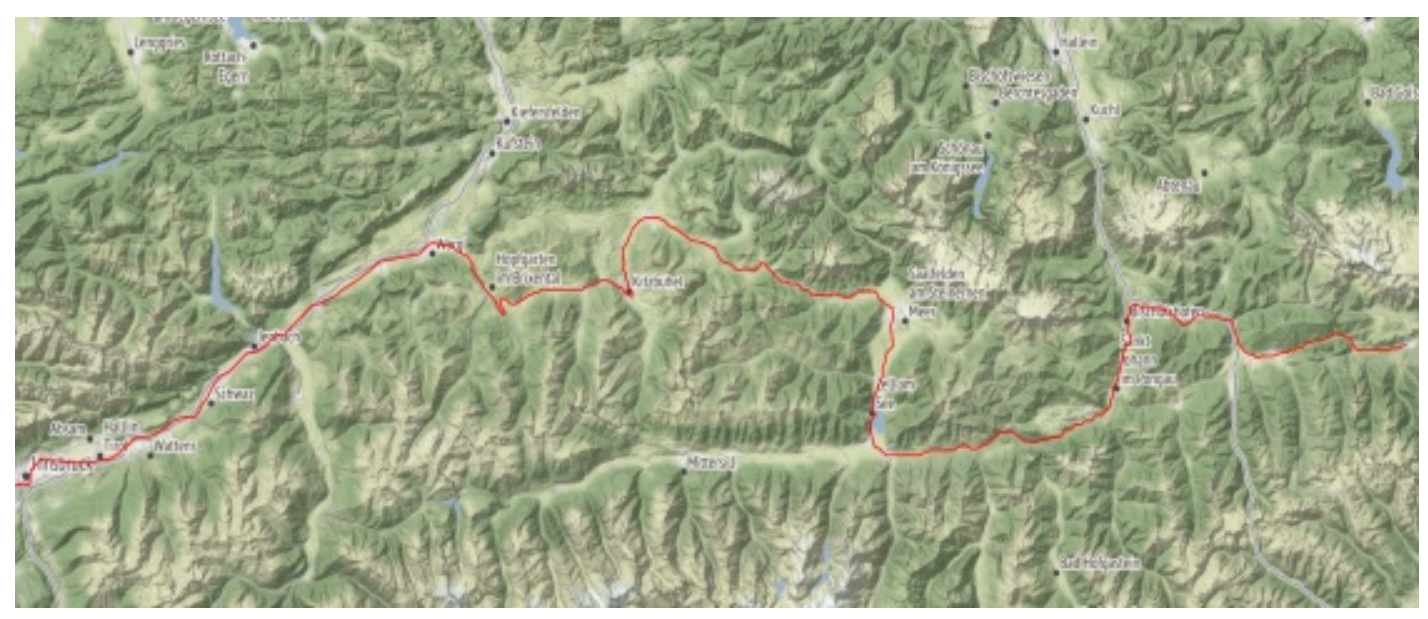

Figure 8.19: Train track of CS3 (29.11.2019): Innsbruck - Woergl - Bischofshofen - Liezen

\subsubsection{Raw data quality}

Figure 8.20 shows the computed CMC observables along the route. Although the magnitudes are quite constant on a medium level (between $+/-5 \mathrm{~m}$ ) and comparable to the prior test cases, some larger spikes are visible especially in the first section (likely urban environment in Innsbruck) and from 13:00 UTC on, where the train enters more mountainous regions.

Figure 8.21 shows the number of observed satellites and computed GDOP along the track, providing a first estimation of the satellite geometry along this route. Looking at values of both available satellites and GDOP, one notices that the first section (more or less the Inn Valley) provides better geometry (GDOP $<2$ for the most part) and visibility of satellites (number of visible satellites constantly over 10 ) whereas these parameters 


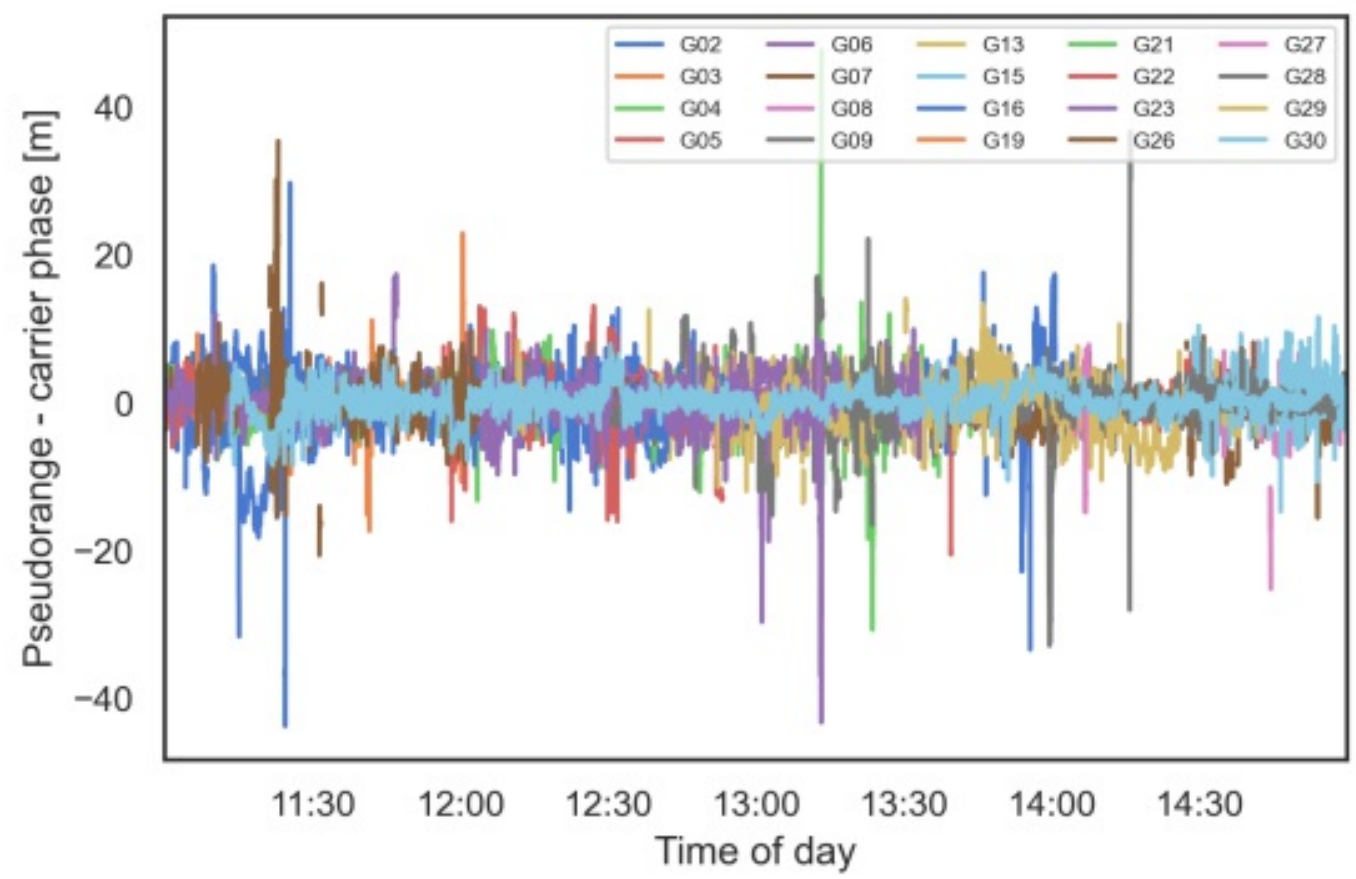

Figure 8.20: $C M C$ observables for CS3, shown are all visible GPS satellites.

are degraded when entering more mountainous environments afterwards.
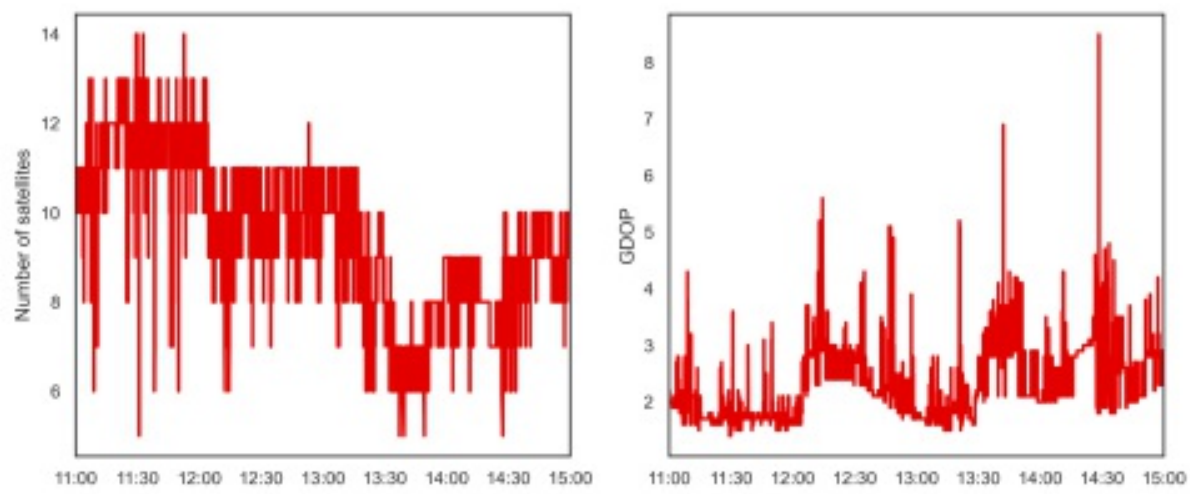

Figure 8.21: Number of visible satellites (left) and GDOP values (right) along the CS3 route. 


\subsubsection{PPP noise analysis}

Analysis of the parameter noise levels reveals similar results as for the prior cases for the most part. Figures 8.22 and 8.23 show the comparison between original PPP output and smoothed solution for CSRS-PPP (top) and PPP-Wizard (bottom).

As for the other test dates the general parameter noise of the solutions is quite low, except for a few larger deviations (spikes) which again directly match with higher multipath levels observed in Figure 8.20. The height solutions are able to describe the frequent terrain/elevation changes of the covered route realistically.
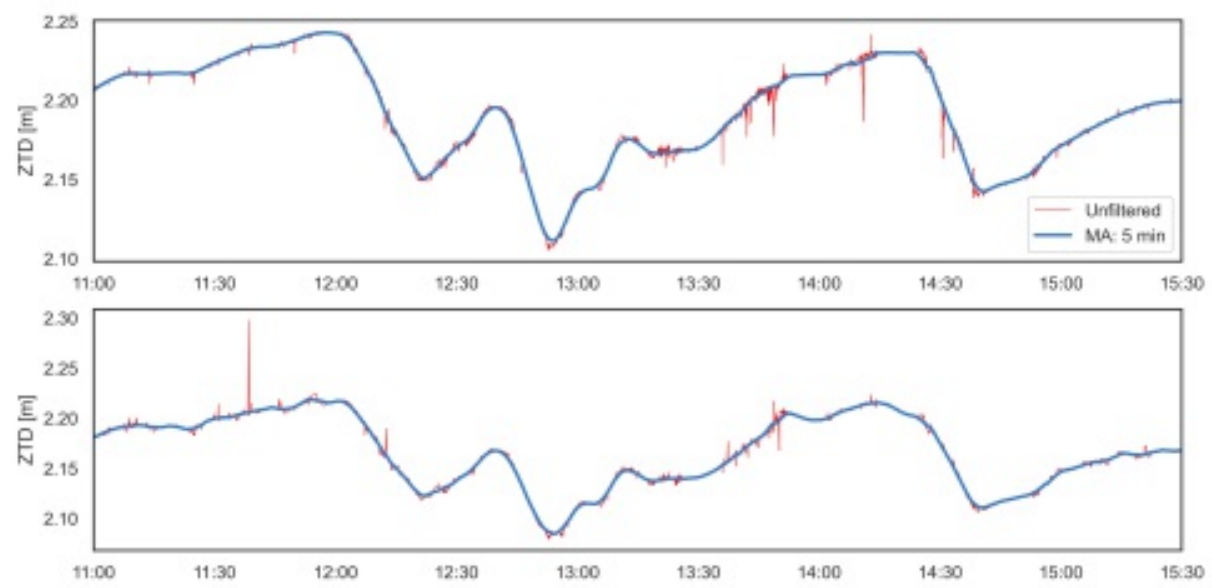

Figure 8.22: CS3 PPP processing results for ZTD from CSRS-PPP (top) and PPPW (bottom). The actual PPP output is shown in red, the MA-smoothed (Interval $5 \mathrm{~min}$ ) in blue.

One main difference to the former cases is the fact that the PPPW solution is more comparable to CSRS-PPP concerning noise level of both, ZTD and height coordinate. Improvements when using CSRS-PPP instead of PPPW are only about a factor 1.5 2 for CS3. This fact also manifests later in the ZTD validation, as this also seems to influence the bias of the ZTD solution.

Bias values are almost equal for both schemes, especially in ZTD. However, the height coordinates show an increased bias (by a factor $\sim 10$ ) for both solutions (decimetre-level) compared to the prior case studies. Interestingly, this behaviour is not mapped to the ZTD biases which are as low as before $\left(\sim 10^{-4} \mathrm{~cm}\right)$.

In general, the magnitude of parameter noise is smaller than for the previous cases. This may be attached to the route characteristics or to possible usage of new equipment (receiver, antenna), as the data from this test case was gathered two years after those of CS1 and CS2. However these assumptions were not investigated further but might 

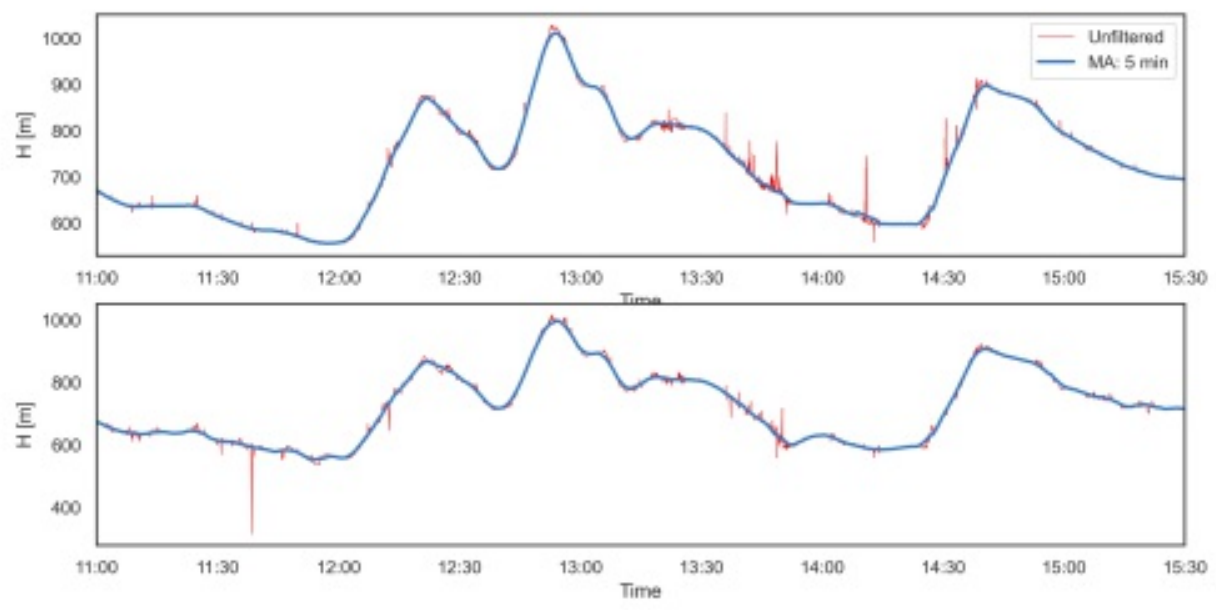

Figure 8.23: CS3 PPP ellipsoidal height time series from CSRS-PPP (top) and PPPW (bottom). The raw PPP output is shown in red, the MA-smoothed (Interval $5 \mathrm{~min}$ ) in blue.

be of interest for future studies using similar datasets.

Detailed statistics of the noise analysis for CS3 are given in Table 8.11 and 8.12.

\begin{tabular}{lcccc} 
Processing scheme & Bias [cm] & Standard deviation [cm] & RMS [cm] & Median [cm] \\
\hline CSRS-PPP & -0.004 & 0.7 & 0.7 & -0.001 \\
PPP-Wizard & -0.005 & 1.2 & 1.2 & -0.003
\end{tabular}

Table 8.11: Performance metrics for the PPP noise analysis of CS3 for CSRS-PPP and PPPW. Measures are computed for ZTD differences between original PPP output and smoothed solution.

\begin{tabular}{lcccc} 
Processing scheme & Bias $[\mathrm{m}]$ & Standard deviation [m] & RMS [m] & Median [m] \\
\hline CSRS-PPP & 0.154 & 23.343 & 23.323 & 0.010 \\
PPP-Wizard & 0.188 & 39.974 & 39.938 & 0.013
\end{tabular}

Table 8.12: Performance metrics for the PPP noise analysis of CS3 for CSRS-PPP and PPPW. Measures are computed for height coordinate differences between original PPP output and smoothed solution.

\subsubsection{ZTD validation}

As with the prior cases, the validation of ZTD estimates is conducted using ZTD computed from ERA5 fields. Figure 8.24 shows the comparison of GNSS-ZTD and ERA5ZTD for this test track. Results show an excellent performance by CSRS-PPP, with 

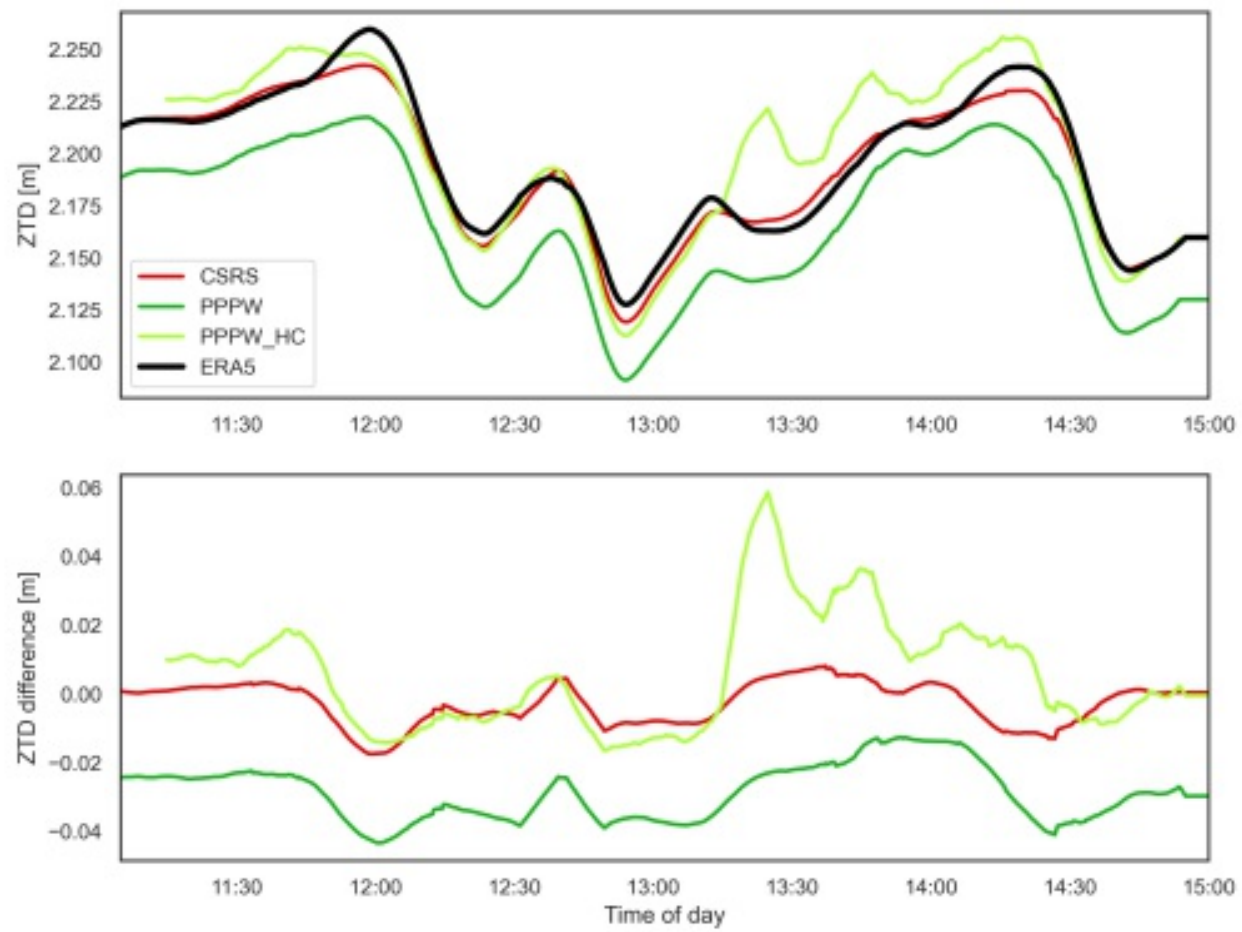

Figure 8.24: Comparison between estimated GNSS-ZTD (CSRS-PPP (red) and PPPW (green)) and ERA5-ZTD (black) for CS3 (29.11.2019), Top: ZTD, bottom: differences between GNSS-ZTD with respect to ERA5-ZTD

differences to ERA5 often in the mm-range and very high correlation between the respective time series. The overall bias is only around $2.7 \mathrm{~mm}$, the median even in the sub-millimetre range and also the standard deviation is the lowest of all schemes (6.1 $\mathrm{mm})$.

PPPW-HC has comparable results for a large part of the route but some larger deviations to ERA5-ZTD can be seen around 13:30 to 14:30 which corresponds to the time with the worst geometry (Figure 8.21). Still, these issues are more likely attached to problems in the track DB heights or originate from interpolation errors made in the constraining process (wrong point/height stored in database is chosen). This becomes clear by looking at the PPPW solution which actually shows a similar pattern as ERA5-ZTD, also in this section.

Overall indeed, the PPPW standard version again shows an increased bias (by a factor 5-10) and RMS compared to CSRS-PPP and PPPW-HC, although this bias is generally lower than for the prior test cases. 
Correlation with ERA5-ZTD is again very high for most schemes, as visible from Figure 8.26. Only PPPW-HC results are distorted by the deviations around 13:30 -14:30 ( $R$ $=0.72$ for PPPW-HC compared to $\mathrm{R}=0.97 / 0.98$ for CSRS-PPP/PPPW). Detailed statistics for the ZTD validation of CS3 can be found in Table 8.13.

Overall the results are again very promising, especially for CSRS-PPP and PPPW-HC, considering the challenging conditions of the route.

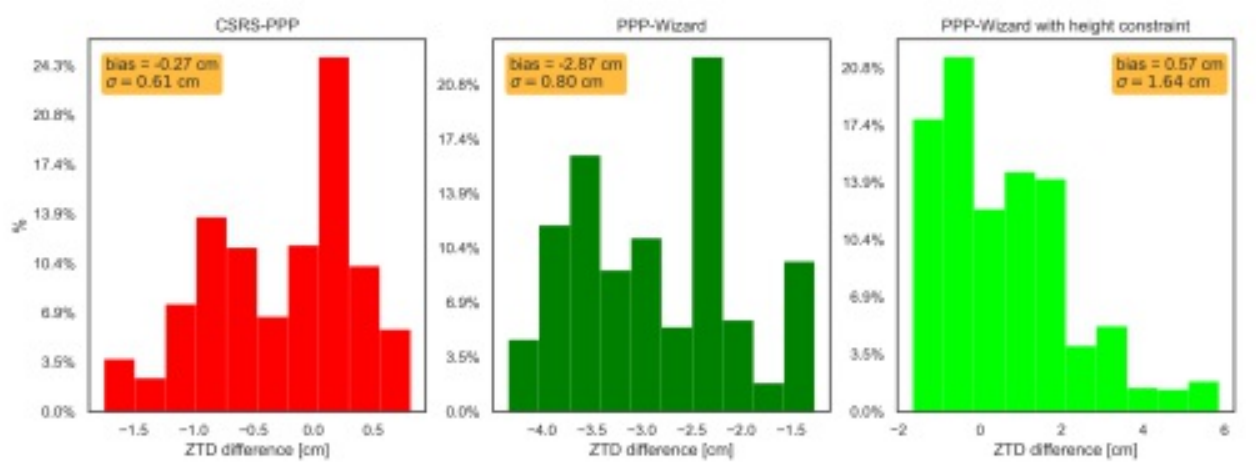

Figure 8.25: Histograms of ZTD differences between GNSS-ZTD and ERA5-ZTD for test case CS3. Left: CSRS, Right: PPPW. Also indicated are bias and standard deviation $(\sigma)$ of the respective solutions. 

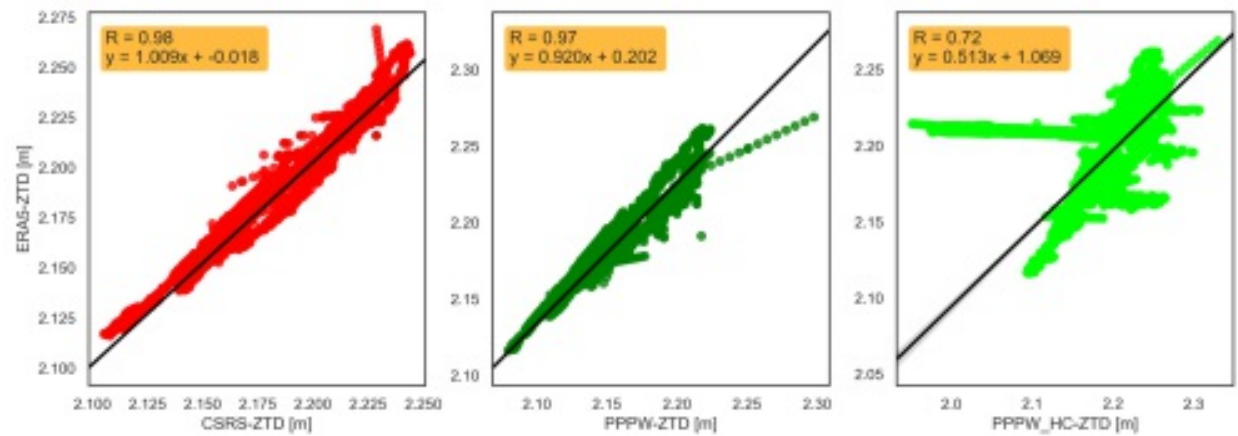

Figure 8.26: Correlation between GNSS-ZTD as estimated by each software package and the ERA5 reference values for CS3, 29.11.2019: (left) CSRS-PPP, (middle) PPPW and (right) PPPW-HC. Terms R and y represent the correlation coefficient and the linear fit, respectively.

\begin{tabular}{lcccc} 
Processing scheme & Bias [cm] & Standard deviation [cm] & RMS [cm] & Correlation \\
\hline CSRS-PPP & -0.27 & 0.61 & 0.66 & 0.98 \\
PPPW & -2.87 & 1.64 & 2.95 & 0.97 \\
PPPW-HC & 0.57 & 0.80 & 1.68 & 0.72
\end{tabular}

Table 8.13: CS3: Performance metrics for the ZTD validation against ERA5-ZTD for processing results of CSRS-PPP, PPPW and PPPW-HC.

\subsection{Case study 4: 01.12.2019}

The fourth and last test case $(01.12 .2019)$ uses data from a railjet train moving on a route very similar to the one of CS3, except that from Liezen the train travelled on to Graz. It is also worth mentioning that the analysed time series represents a night travel, which might account for a smaller ionospheric influence on the results.

As mentioned with CS3, the route provides diverse conditions with a large number prominent features along the track.

The exact travelling track, as derived from CSRS-PPP processing, is visualized in Figure 8.27 .

\subsubsection{Raw data quality}

The analysis of multipath (CMC observables), presented in Figure 8.28, shows a similar picture as for CS3 for the most part. Some larger spikes are visible, most prominently around 02:30 UTC and in the last part of the route. As for CS3, they correspond 


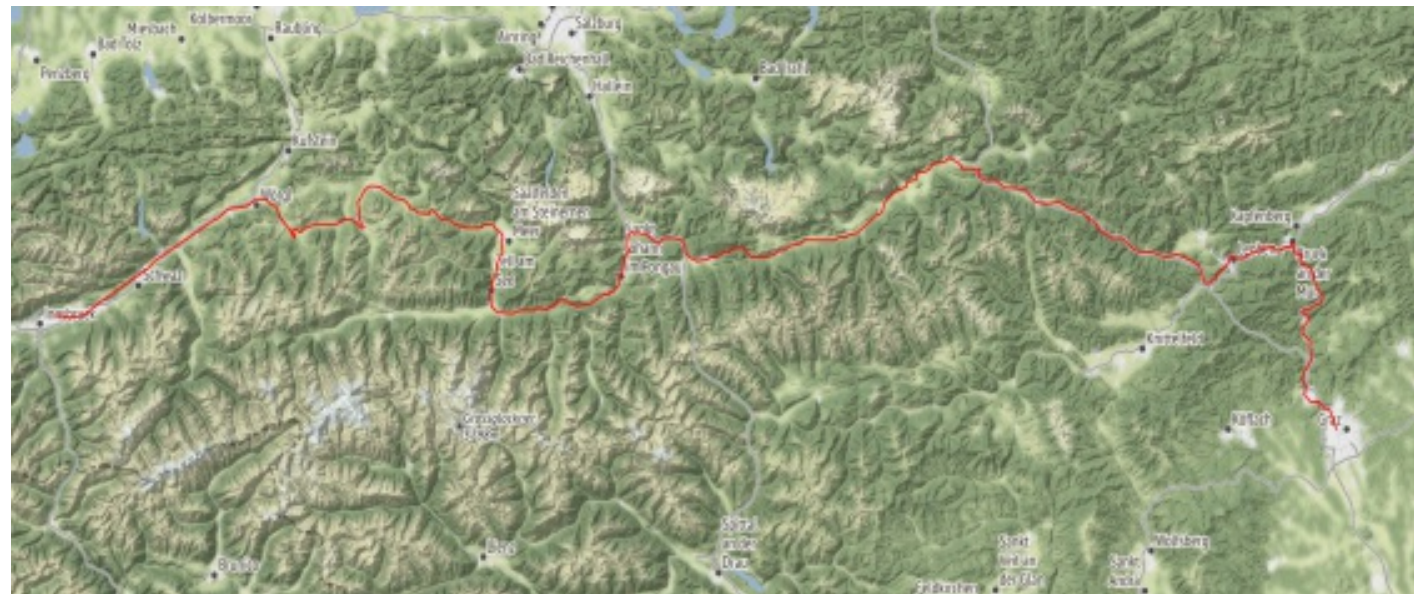

Figure 8.27: Train track of CS4 (01.12.2019): Innsbruck - Woergl - Bischofshofen - Liezen Leoben - Bruck/Mur - Graz

approximately with times when (1) a highly mountainous area and (2) additionally more urban areas (when the train approaches Graz) are entered. This picture is also consistent

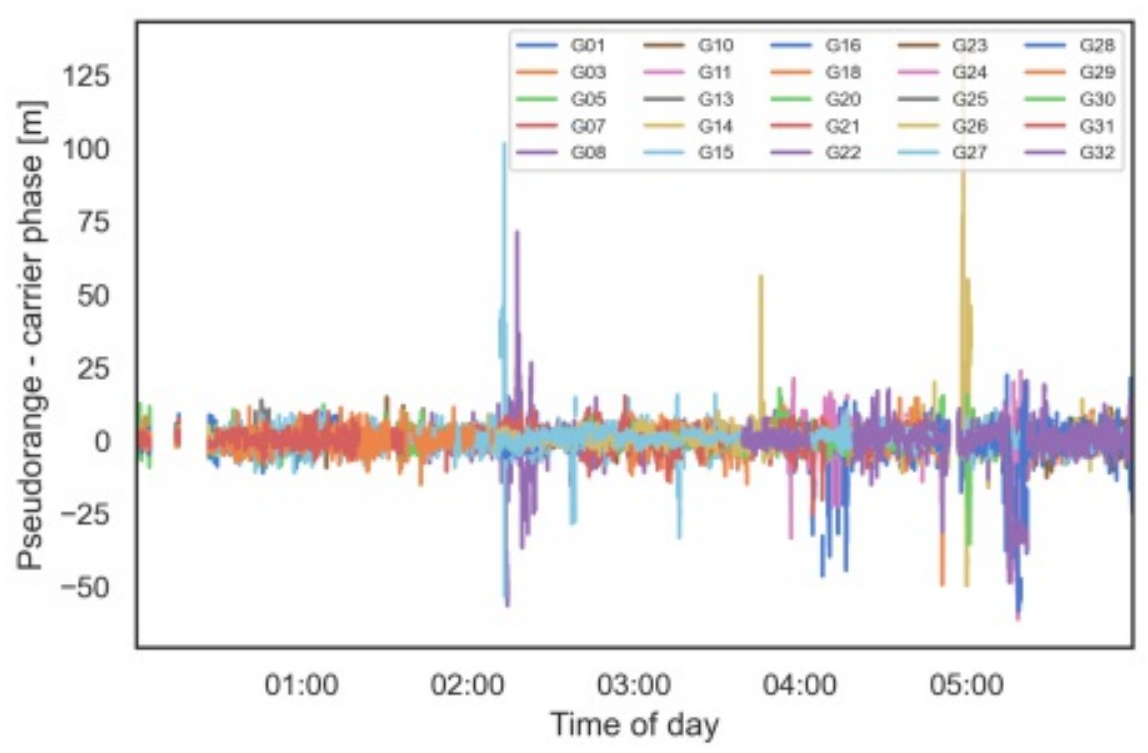

Figure 8.28: CMC observables for CS4, shown are all visible GPS satellites.

with Figure 8.29, which shows the number of observed satellites and GDOP along the track. The problematic areas can again be recognized in terms of higher GDOP values and a drop in the number of available satellites. 

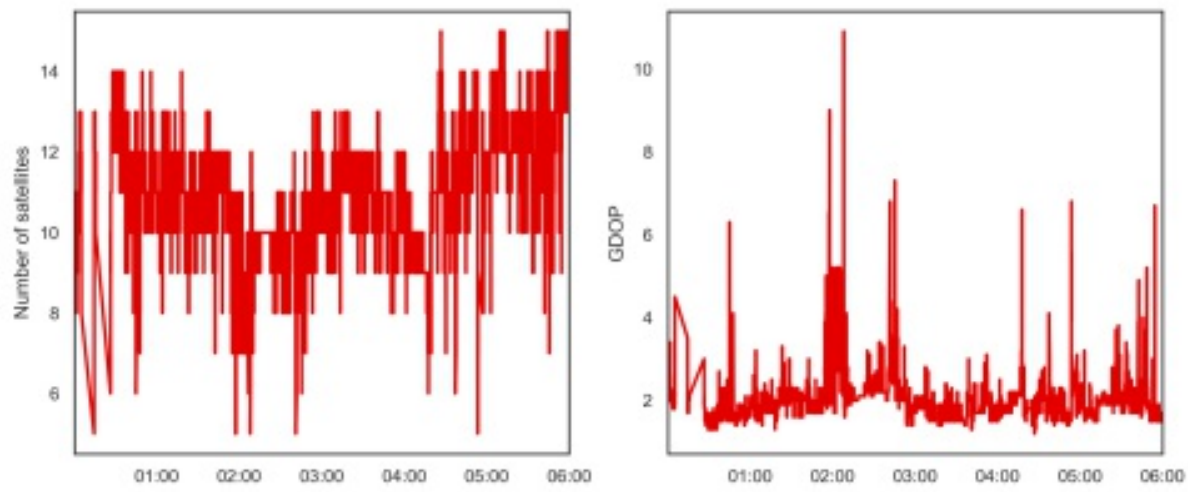

Figure 8.29: Number of visible satellites (left) and GDOP values (right) along the CS3 route.

\subsubsection{PPP noise analysis}

Again for this last test case a noise analysis for the PPP schemes is carried out. Similar to CS3, the overall noise is again low and again the CSRS-PPP solution shows better performance. Bias values are again vanishingly small for ZTD $\left(<10^{-4} \mathrm{~cm}\right)$ and height coordinate (millimetre-level), showing the feasibility of the smoothing time window.

PPPW ZTD results are comparable to those of CSRS-PPP for bias/median but with increased standard deviation and RMS $(1.9 \mathrm{~cm}$ compared to $0.1 \mathrm{~cm})$.

Height coordinate results also reveals RMS and standard deviation values comparable to those derived for the prior test cases and again are lower for CSRS-PPP compared to PPPW. This is also consistent with all prior test cases but the deviations are again smaller than for the first two cases (as for CS3). Possible reasons, as updated equipment (receiver/antenna) for this performance have already been discussed for CS3 but need further research as already mentioned.

\begin{tabular}{lcccc} 
Processing scheme & Bias [cm] & Standard deviation [cm] & RMS [cm] & Median [cm] \\
\hline CSRS-PPP & -0.0001 & 0.1 & 0.1 & -0.0008 \\
PPP-Wizard & -0.0002 & 1.9 & 1.9 & -0.004
\end{tabular}

Table 8.14: Performance metrics for the PPP noise analysis of CS4 for CSRS-PPP and PPPW. Measures are computed for ZTD differences between original PPP output and smoothed solution. 

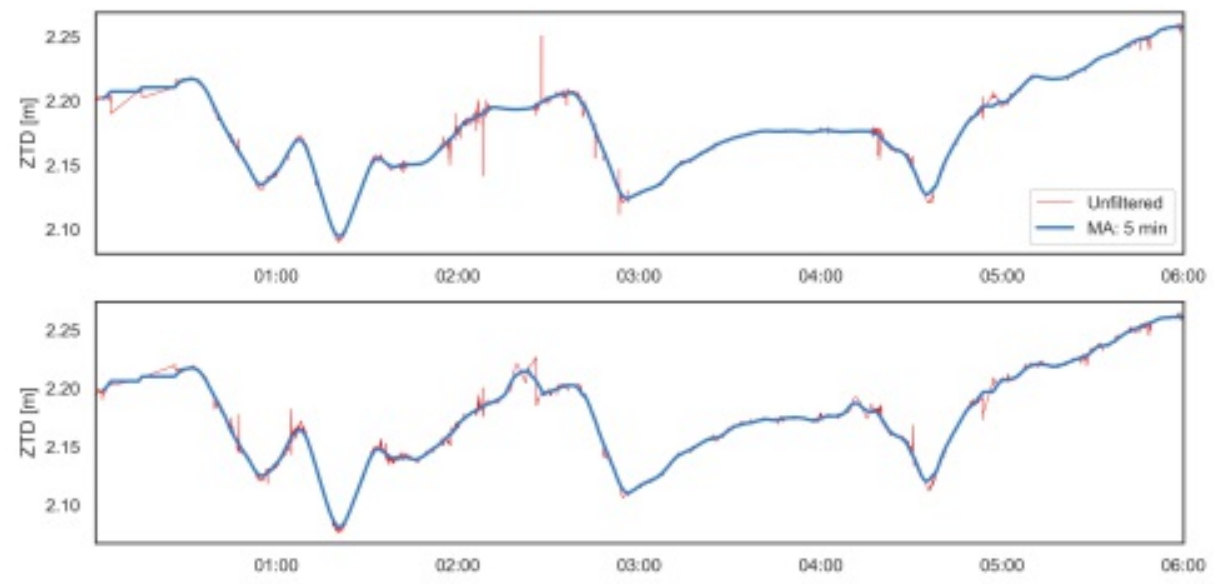

Figure 8.30: CS4 PPP ZTD time series from CSRS-PPP (top) and PPPW (bottom). The raw PPP output is shown in red, the MA-smoothed (Interval $5 \mathrm{~min}$ ) in blue.
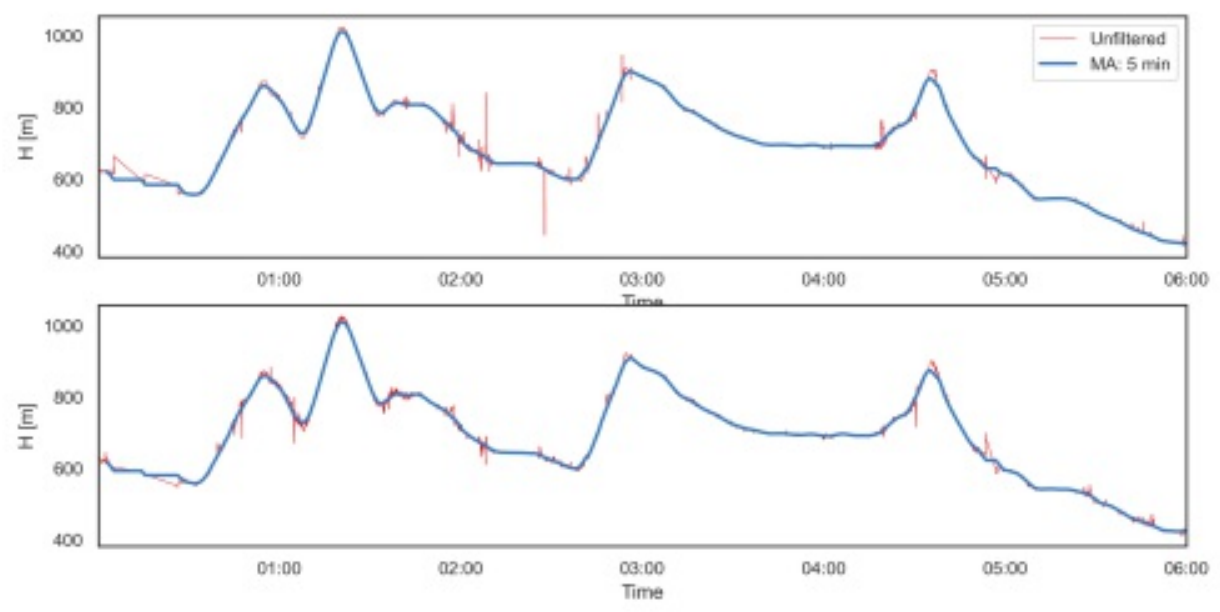

Figure 8.31: CS4 PPP ellipsoidal height time series from CSRS-PPP (top) and PPPW (bottom). The raw PPP output is shown in red, the MA-smoothed (Interval $5 \mathrm{~min}$ ) in blue.

\begin{tabular}{lcccc} 
Processing scheme & Bias $[\mathrm{m}]$ & Standard deviation [m] & RMS [m] & Median [m] \\
\hline CSRS-PPP & 0.007 & 3.969 & 3.972 & -0.001 \\
PPP-Wizard & 0.005 & 13.552 & 13.564 & 0.018
\end{tabular}

Table 8.15: Performance metrics for the PPP noise analysis of CS4 for CSRS-PPP and PPPW. Measures are computed for height coordinate differences between original PPP output and smoothed solution. 


\section{Comparison with RTK}

CS4 (unfortunately) represents the only test case where a RTK-derived coordinate solution is available and therefore allows to compare its height coordinate solution to those derived from CSRS-PPP and PPPW. The RTK solution was also provided by ÖBB, together with the raw observations for this test case. It has a similar update rate as the PPP solution $(1 \mathrm{~Hz})$.

This comparison is presented in Figure 8.32 which shows time series of ellipsoidal height from CSRS-PPP (red) and PPPW (green) as well as the RTK solution (black). Comparison of original processing output is shown in the top part as well as comparison for the MA-smoothed time series in the bottom part of Figure 8.32.
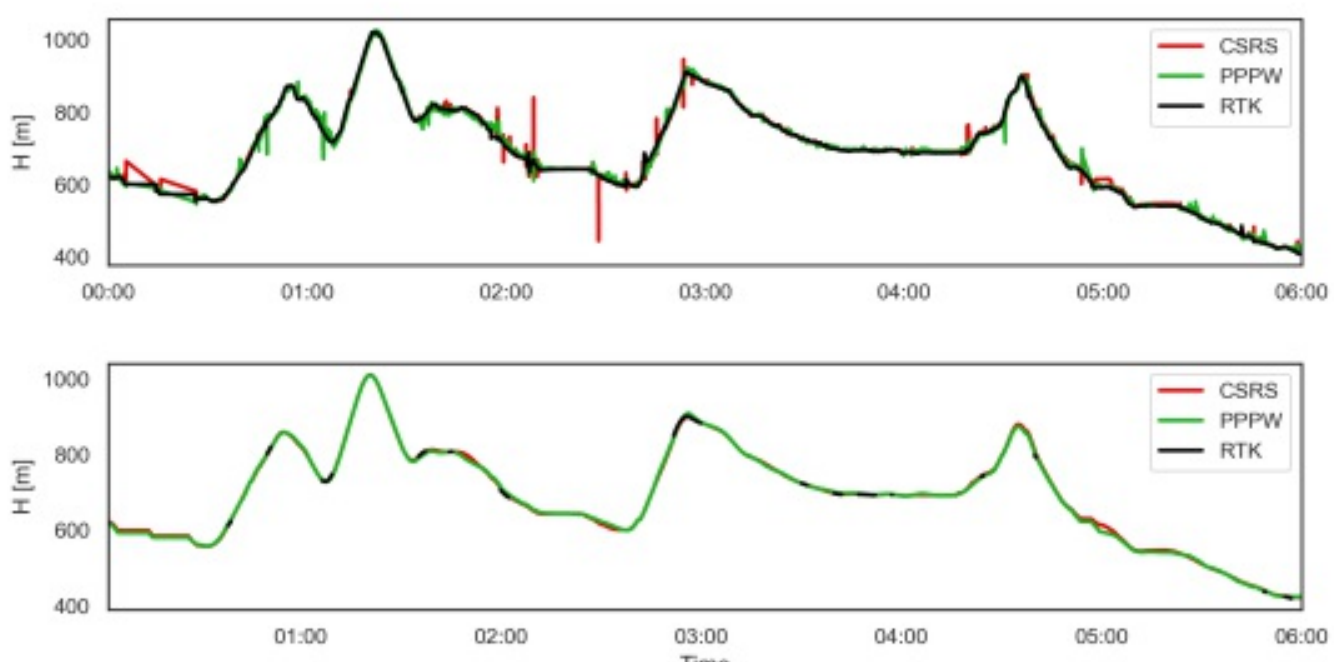

Figure 8.32: Comparison of ellipsoidal height between PPP and RTK solutions for CS4 (01.12.2019): CSRS-PPP (red) / PPP-Wizard (green) / RTK solution (black). Original processing output time series shown on top, MA-filtered time series at the bottom.

Results in form the usual performance metrics for differences between the PPP heights and the RTK heights are presented in Tables 8.16 (original output) and 8.17 (filtered time series). Comparing the results shown in the tables, the huge benefits of the smoothing become apparent. All performance metrics are improved (up to factor 5-10, on average by factor $\sim 2$ ) by applying the MA-filter as described in Section 8.1. Filtered time series show deviations (bias and median) in the decimetre-range for CSRS-PPP and low metre-range for PPPW, which correspond to the current limits (as presented in literature) for both PPP and RTK in such highly kinematic scenarios.

Comparing the original output of RTK and PPP solutions yields bias, RMS and median values of approximately 2-2.5 metres and thus shows higher values than the expected 
quality range for kinematic SF-PPP processing (decimetre-range). Reasons for this substantial differences might be the different ionospheric mitigation strategies for SF-PPP and RTK (although corrections using a TEC model are already applied for PPP). Another possible explanation is that the RTK solution might not be referring to the GNSS antenna but the actual receiver position (inside the train). Since no detailed documentation was provided for the RTK data these assumptions could not be investigated further. Moreover, one notices PPPW showing better agreement for the comparison of original processing output with RTK whereas CSRS-PPP shows smaller deviations in the case of smoothed time series.

Overall these comparisons outline a good PPP performance and show results comparable to the RTK height coordinate solution, which also increases the confidence in the ZTD results derived from PPP processing.

\begin{tabular}{lcccc} 
Processing scheme & Bias [m] & Standard deviation [m] & RMS [m] & Median [m] \\
\hline CSRS-PPP & 2.449 & 6.782 & 7.397 & 2.162 \\
PPPW & 1.995 & 7.626 & 7.903 & 2.046
\end{tabular}

Table 8.16: Performance metrics for the PPP noise analysis of CS4 for CSRS-PPP and PPPW. Measures are computed for height coordinate differences between raw PPP output and the raw RTK solution.

\begin{tabular}{lcccc} 
Processing scheme & Bias [m] & Standard deviation [m] & RMS [m] & Median [m] \\
\hline CSRS-PPP & -0.048 & 3.229 & 3.229 & 0.267 \\
PPPW & 1.538 & 4.517 & 4.772 & 1.505
\end{tabular}

Table 8.17: Performance metrics for the PPP noise analysis of CS4 for CSRS-PPP and PPPW. Measures are computed for height coordinate differences between smoothed PPP and RTK time series. 


\subsubsection{ZTD validation}

The ZTD validation for CS4 provides some interesting results which deviate to some extend from the former cases. Its main results are shown in Figures $8.33-8.35$.
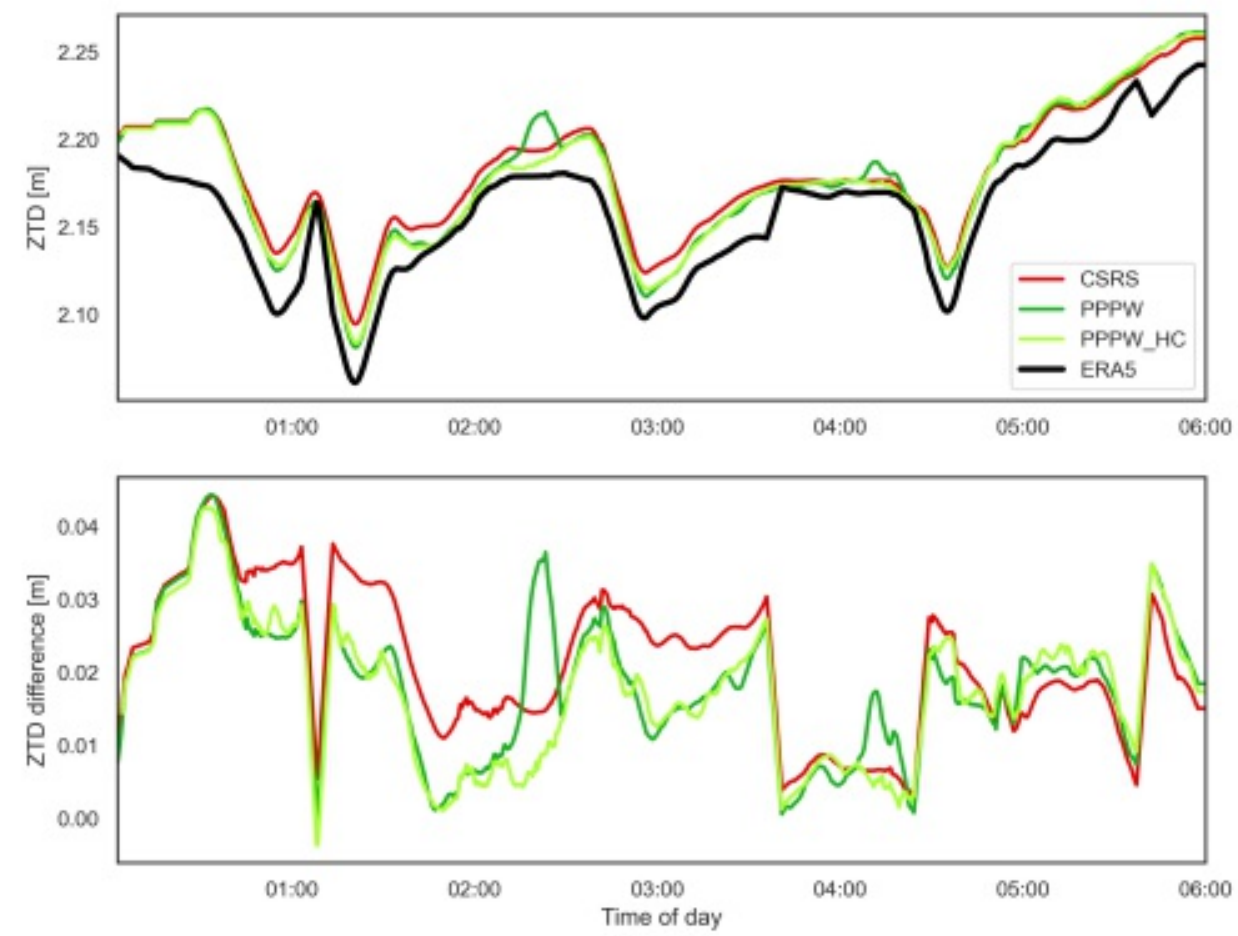

Figure 8.33: Comparison between estimated GNSS-ZTD (CSRS-PPP (red) and PPP-Wizard (green)) and ERA5-ZTD (black) for CS4 (01.12.2019), Top: absolute values, bottom: differences between GNSS-ZTD with respect to ERA5-ZTD

Overall, the results are again very promising, with highest bias and RMS values in the low centimetre-range.

The main deviation to prior test cases is the relative performance of the processing schemes. Especially surprising is the fact that in this case CSRS-PPP provides the least accurate results, based on the validation with ERA5-ZTD. Furthermore, the standard PPPW this time shows lowest standard deviation of all three schemes. As for CS2, the PPPW-HC processing performs best in terms of bias $(1.64 \mathrm{~cm})$ and RMS $(1.87 \mathrm{~cm})$ but improvements compared to the standard version (PPPW) are marginal this time. Correlation of all schemes with ERA5-ZTD is again very high and the standard deviations $(\sim 0.8-1.1 \mathrm{~cm})$ are comparable to CS3, see Figure 8.35.

A detailed summary of the statistics discussed above is provided in Table 8.18. 


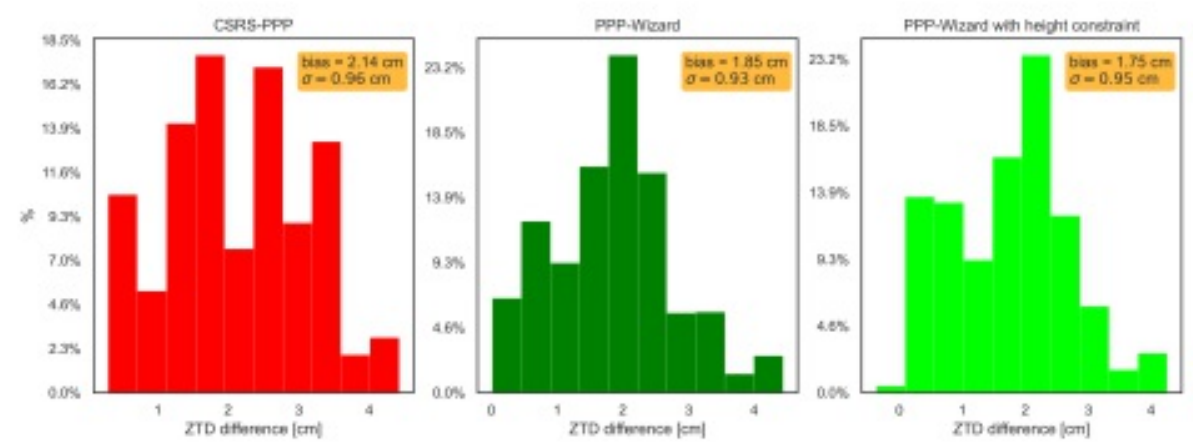

Figure 8.34: Histograms of ZTD differences between GNSS-ZTD and ERA5-ZTD for test case CS4. Left: CSRS, Right: PPP-Wizard. Also indicated are bias and standard deviation $(\sigma)$ of the respective solutions.
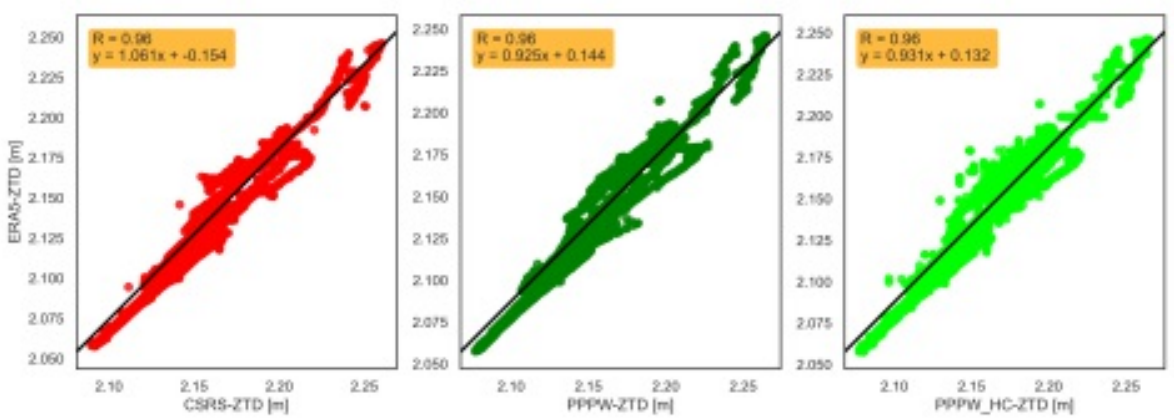

Figure 8.35: Correlation between ZTD as estimated by each software package and the ERA5 reference values for CS4, 01.12.2019: (left) CSRS-PPP, (middle) PPPW and (right) PPPWHC. Terms $\mathrm{R}$ and y represent the correlation coefficient and the linear fit, respectively.

\begin{tabular}{lcccc} 
Processing scheme & Bias [cm] & Standard deviation [cm] & RMS [cm] & Correlation \\
\hline CSRS-PPP & 2.08 & 0.97 & 2.20 & 0.96 \\
PPPW & 1.77 & 0.85 & 1.89 & 0.96 \\
PPPW-HC & 1.64 & 1.07 & 1.87 & 0.96
\end{tabular}

Table 8.18: CS4: Performance metrics for the ZTD validation against ERA5-ZTD for processing results of CSRS-PPP, PPPW and PPPW-HC. 


\subsection{ZTD sensitivity analysis}

In addition to the main results of the four test cases presented before, this section investigates the influence of certain adaptations in the processing settings of the purposed approaches. Some of those changes are of particular interest for NRT processing approaches described in the next chapter. In the following section, only selected software packages (GAMP/PPP-Wizard) are used depending on their capabilities for fine-tuning of the processing. As already mentioned before, CSRS-PPP is not configurable manually and therefore not feasible for such types of studies.

Aim of this investigations is to assess the sensitivity of the ZTD solution conditional on the use of specific data and settings in PPP processing. The following settings are assessed in the course of this section:

- Usage of GLONASS observations

- Elevation cut-off

- Gradient estimation

- Mis-modelling of ionospheric delays using SEID

- Height-dependency of ZTD errors

Compared to the main results from the case studies, a detailed summary of statistical performance measures is not presented for these sensitivity studies. Instead a visual comparison and only a few key metrics as performance indicators are used to emphasize possible benefits for certain changes in processing.

\subsubsection{Impact of GLONASS observations}

In the following, the impact of additional GLONASS observations in PPP processing on the ZTD solution is assessed using PPPW ZTD solutions for CS3 and CS4, processed in GPS+GLONASS and GPS-only PPP mode.

Typically GLONASS observations are of lower quality but can stabilize solutions in critical environments where only less GPS satellites are available.

The results from GPS-only vs GPS+GLONASS processing for the test cases CS3 and CS4 are shown in Figures 8.36 and 8.37 respectively, as for the prior sections in terms of ZTD and differences to ERA5-ZTD. In both cases no significant impact of the additional observations can be found. There are small deviations between the solutions which are visible in the difference plots, for example around 12:30 UTC in Figure 8.36. Most of 
these small deviations even indicate better performance of the GPS-only solution, which might indicate rather poor quality of either GLONASS observations or GLONASS correction products (e.g clock corrections). Bias, standard deviation and RMS values are practically unchanged by adding/removing GLONASS observations from the processing (see Tables 8.19 and 8.20). This disagrees with studies like Webb et al. (2016), who found improvements of about $5 \mathrm{~mm}$ on average when using GLONASS observations additionally. However these studies used data from high-grade DF receivers and environmental conditions were (while still mountainous) not really comparable.

For future applications it might be interesting to analyse the performance of GPS + Galileo solutions as soon as the new generation of receivers is available within the Greenlight project.
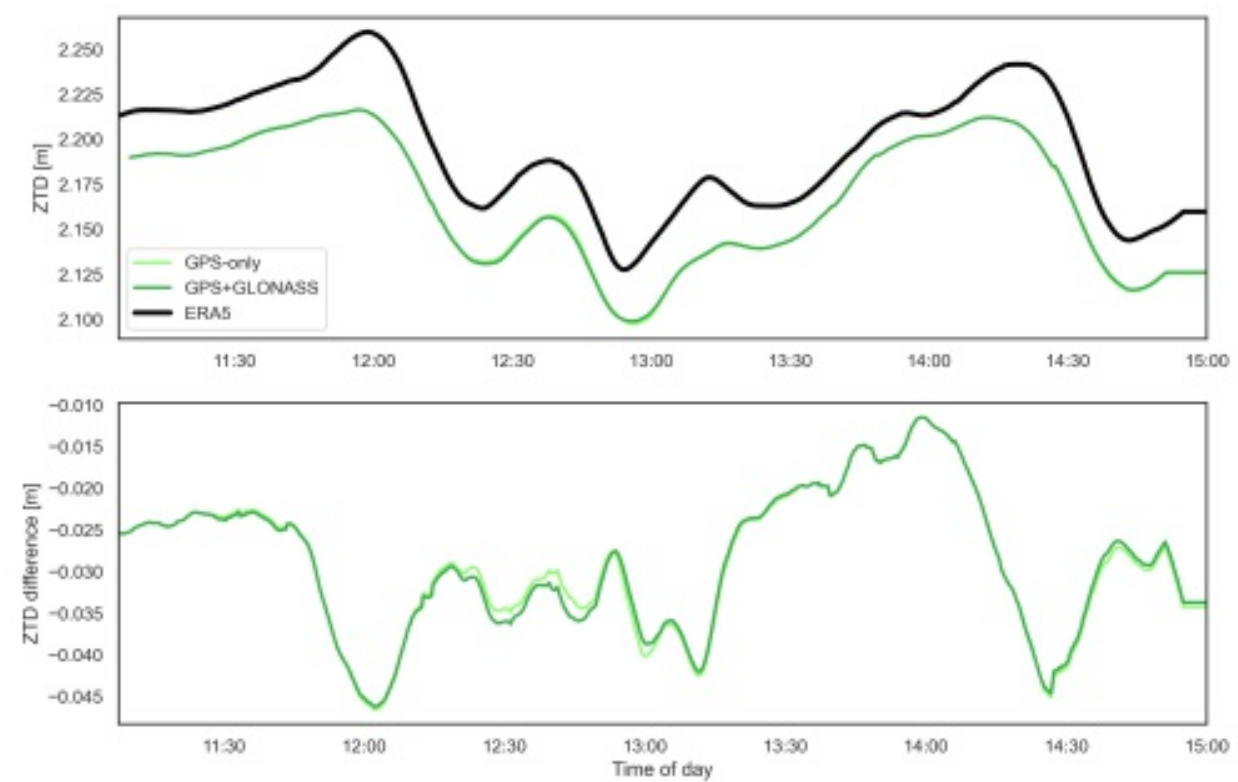

Figure 8.36: Comparison of GNSS-ZTD with ERA5-ZTD with/without GLONASS observations for CS3: (1) GPS+GLONASS (dark green),(2) GPS-only (light green). Absolute ZTD values (top) and absolute differences to ERA5-ZTD (bottom).

\begin{tabular}{lcccc} 
Setting & Bias [cm] & Standard deviation [cm] & RMS [cm] & Correlation \\
\hline GPS-only & -2.88 & 0.84 & 3.00 & 0.97 \\
GPS+GLONASS & -2.89 & 0.83 & 3.00 & 0.97
\end{tabular}

Table 8.19: CS3: Performance metrics for the ZTD validation against ERA5-ZTD for processing results for GPS-only and GPS+GLONASS 

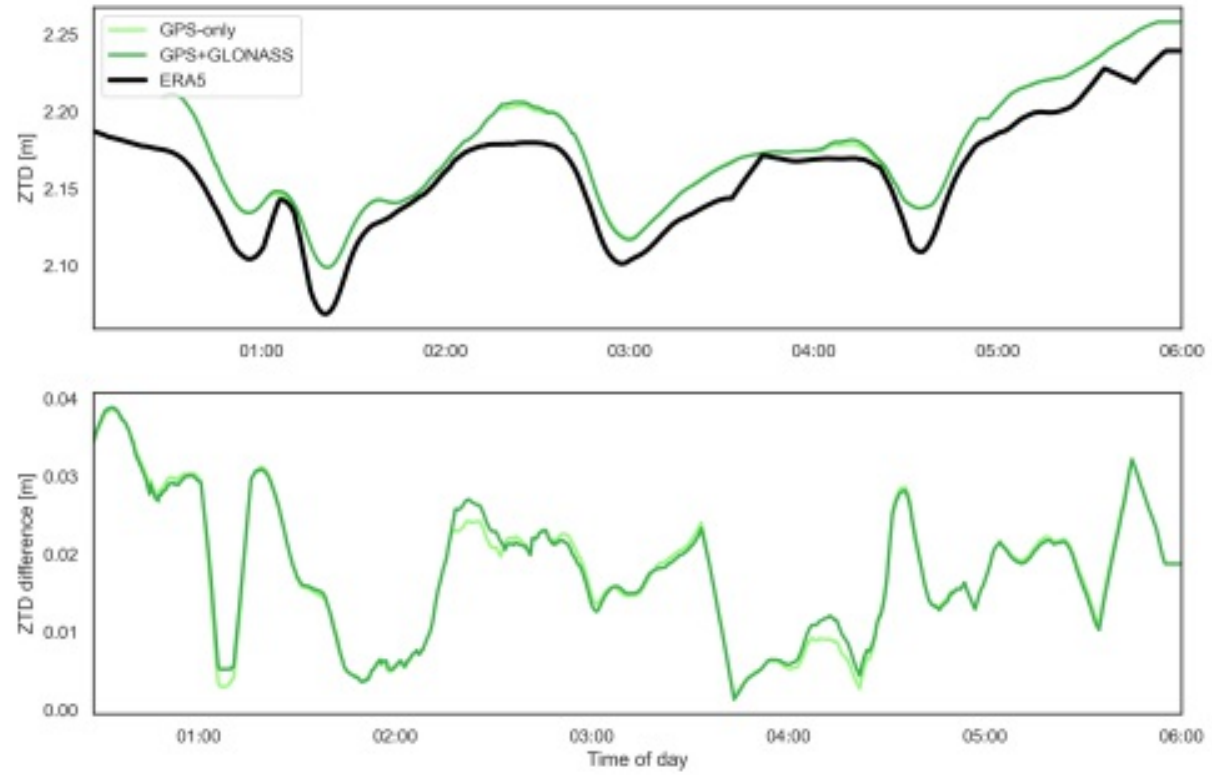

Figure 8.37: Comparison of GNSS-ZTD with ERA5-ZTD with/without GLONASS observations for CS4: (1) GPS+GLONASS (dark green),(2) GPS-only (light green). ZTD values (top) and differences to ERA5-ZTD (bottom).

\begin{tabular}{lcccc} 
Setting & Bias [cm] & Standard deviation [cm] & RMS [cm] & Correlation \\
\hline GPS-only & 1.76 & 0.87 & 1.96 & 0.97 \\
GPS+GLONASS & 1.77 & 0.85 & 1.97 & 0.97
\end{tabular}

Table 8.20: CS4: Performance metrics for the ZTD validation against ERA5-ZTD for processing results for GPS-only and GPS+GLONASS 


\subsubsection{Elevation cut-off}

This section investigates the sensitivity of the ZTD solution to the applied elevation cut-off. The only software package where the elevation cut-off can be varied is GAMP and therefore only test case CS2 is analysed here. Three different solutions are produced for $5^{\circ}, 7.5^{\circ}$ (default) and $10^{\circ}$ cut-off, where the last two versions were found to be identical and therefore are only shown once. ZTD solutions in comparison to ERA-ZTD are presented in Figure 8.38 and the corresponding histograms of the ZTD differences in Figure 8.39.

The results indicate a visible improvement/degradation of the solution with higher/lower cut-off setting. This is most prominent in the flatter/lower-altitude parts of the track (beginning and end sections) which seems intuitive due to the fact that only there satellites at low elevation will be visible/trackable. Largest improvements in those areas are in the cm-range and therefore clearly beneficial for ZTD estimates. The overall bias can be reduced by about $2 \mathrm{~mm}$ and standard deviation is reduced by $5.5 \mathrm{~mm}$ for the higher elevation cut-off solutions (see Table 8.21). Moreover it is visible from Figure 8.39, that the computed differences to ERA5-ZTD follow the expected Gaussian distribution to a larger degree for the higher cut-off. The sample size of ZTD differences might be relativity small but nevertheless a trend of improvement is already noticeable.

However, over a small part on the track between 10:00 and 10:30 the opposite behaviour can also be recognized. Most likely this is due to an already considerably low number of satellites in view, which could already be seen from Figure 8.12 in Section 8.3.

These results may disagree with common strategies used for tropospheric estimation from GNSS, where using lower cut-off settings achieves a better decorrelation of ZTD from the height coordinate and therefore is expected to provide improved results. A more detailed discussion on this topic is given in the final conclusions in Chapter 10.

\begin{tabular}{lcccc} 
Setting & Bias [cm] & Standard deviation [cm] & RMS [cm] & Correlation \\
\hline Cut-off $\mathbf{5}^{\circ}$ & 0.54 & 2.55 & 2.56 & 0.94 \\
Cut-off $\mathbf{7 . 5 / 1 0}$ & 0.38 & 2.01 & 2.01 & 0.96
\end{tabular}

Table 8.21: Performance metrics for the ZTD validation against ERA5-ZTD for processing results for $5^{\circ}$ and $7.5 / 10^{\circ}$ cut-off elevation angle settings. Investigated test case is CS2. 

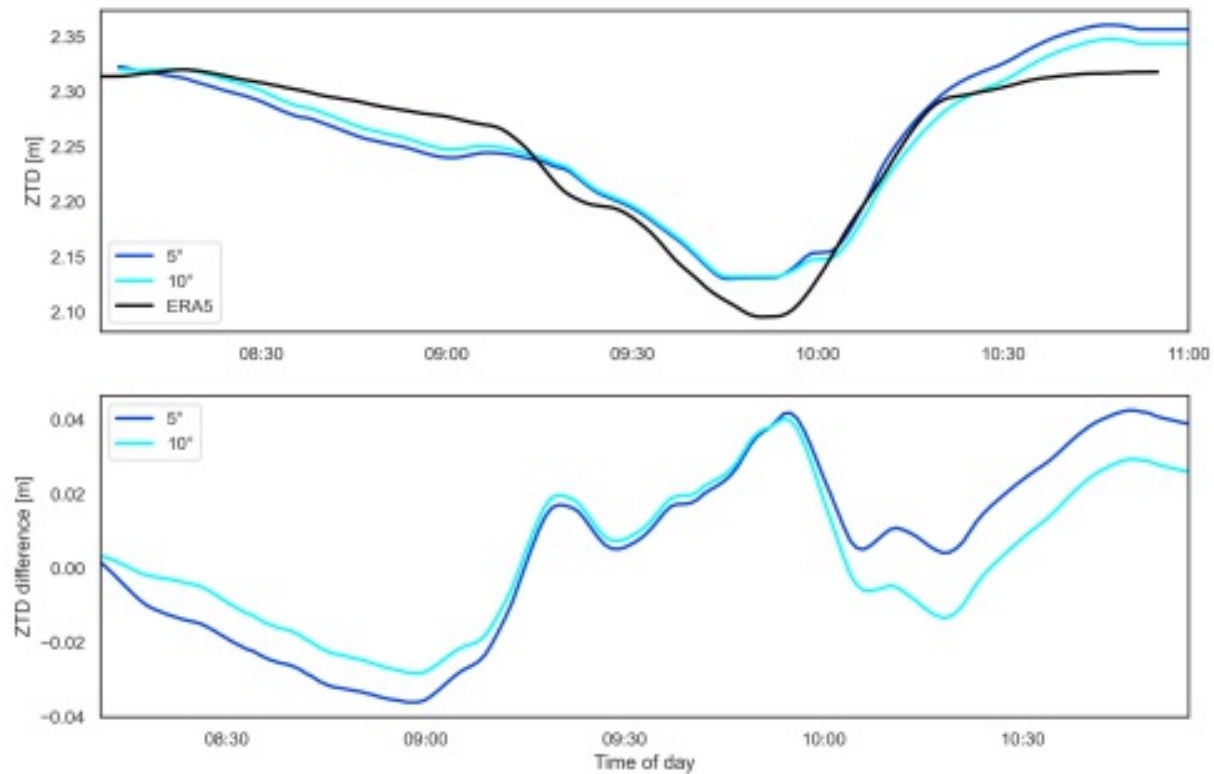

Figure 8.38: Comparison of GNSS-ZTD with ERA5-ZTD for different settings for elevation cut-off: (1) $5^{\circ}$ (dark blue),(2) $10^{\circ}$ (light blue). Absolute ZTD values (top) and absolute differences to ERA5-ZTD (bottom).
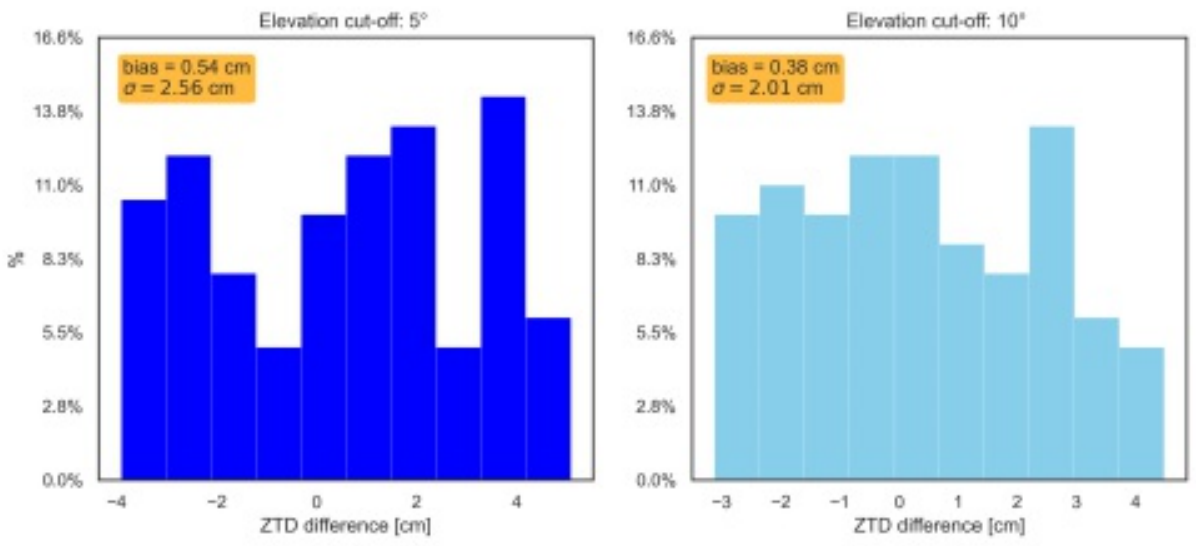

Figure 8.39: Histograms of GNSS-ZTD differences to ERA5-ZTD for different settings for elevation cut-off: (1) $5^{\circ}$ (dark blue),(2) 7.5/10 (light blue). 


\subsubsection{Estimation of horizontal gradients}

This section investigates the possible benefit of additionally estimating horizontal tropospheric gradients in PPP processing.

The extension of the parameter estimation for tropospheric gradients is a typical procedure in GNSS Meteorology. Many studies have confirmed its beneficial effects on troposphere and/or position solutions and their usability for data assimilation. Furthermore they also enable the user to calculate slant delays for each observed satellite, a parameter which is of increasing interest in the scientific community nowadays.

However, in a situation like present within this study the expected benefits of estimating this additional parameter might be reduced or not present at all. Some detailed thoughts on this topic can be found in the next chapter, Section 9.4

However it should be mentioned that the results shown in the following only assess the influence of additional gradient estimation on the ZTD solution. No actual gradient parameters are analysed, nevertheless this might be a topic for future investigations/applications.

Gradients were introduced in processing using the formulation of Chen and Herring (1997) and estimated every epoch together with the common parameters (coordinates, receiver clock error and ZTD). The results from ZTD-only vs ZTD and gradients (ZTD+GRAD) processing for CS2 are shown in Figure 8.40 and the respective histogram of the differences to ERA5-ZTD in Figure 8.41.

As with the usage of GLONASS, no significant impact can be found and distributions of ZTD differences are practically unchanged by additional gradient estimation (see Table 8.22) as indicated also in the visual comparison of the ZTD (difference) time series. This disagrees with most literature on this topic, a fact that might be attached to the challenging conditions present in this study (especially for CS2 which is analysed here). For large parts of the analysed track, satellite visibility is reduced and redundancy might not be sufficient to estimate gradient parameters of good quality or increase the quality of estimated ZTDs.

\begin{tabular}{lcccc} 
Setting & Bias [cm] & Standard deviation [cm] & RMS [cm] & Correlation \\
\hline ZTD-only & 0.66 & 2.58 & 2.54 & 0.94 \\
ZTD+GRAD & 0.68 & 2.58 & 2.54 & 0.94
\end{tabular}

Table 8.22: Performance metrics for the ZTD validation against ERA5-ZTD for processing results for ZTD-only and ZTD+GRAD settings. Investigated test case is CS2. 

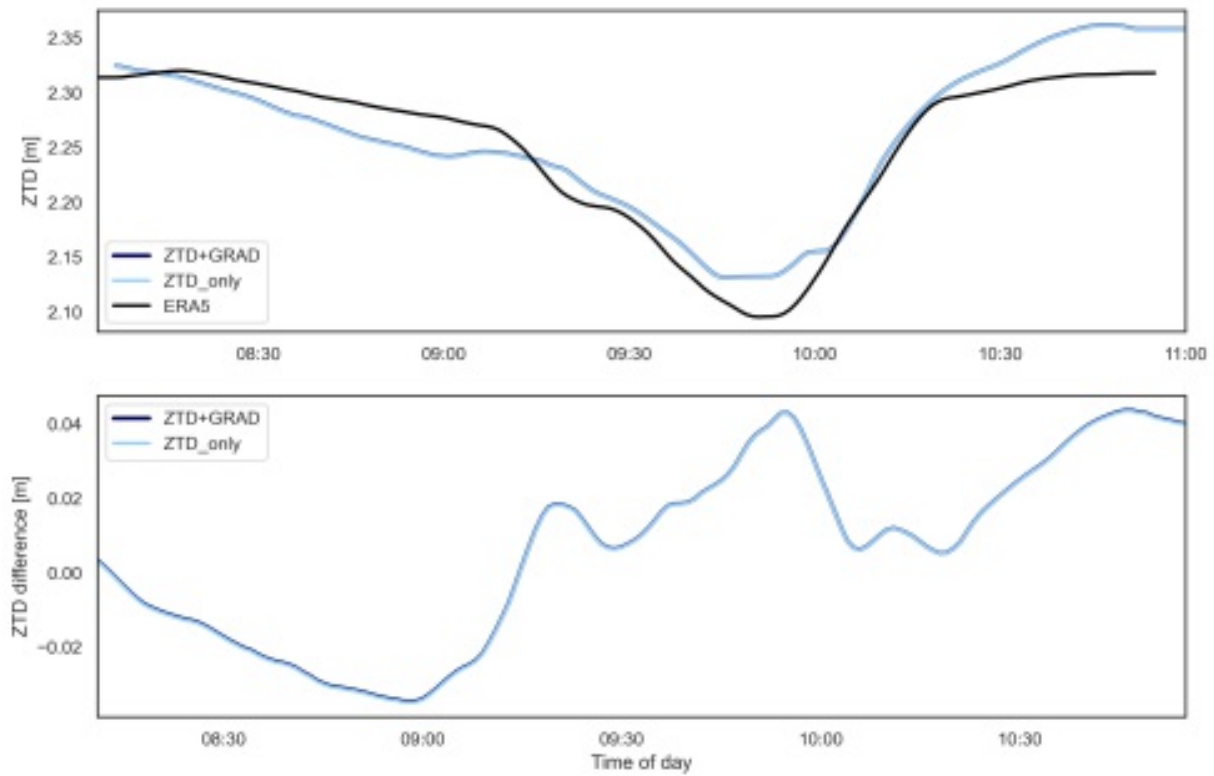

Figure 8.40: Comparison of GNSS-ZTD with ERA5-ZTD with/without gradient estimation: (1) ZTD+GRAD (dark blue),(2) ZTD-only (light blue). Absolute ZTD values (top) and absolute differences to ERA5-ZTD (bottom).
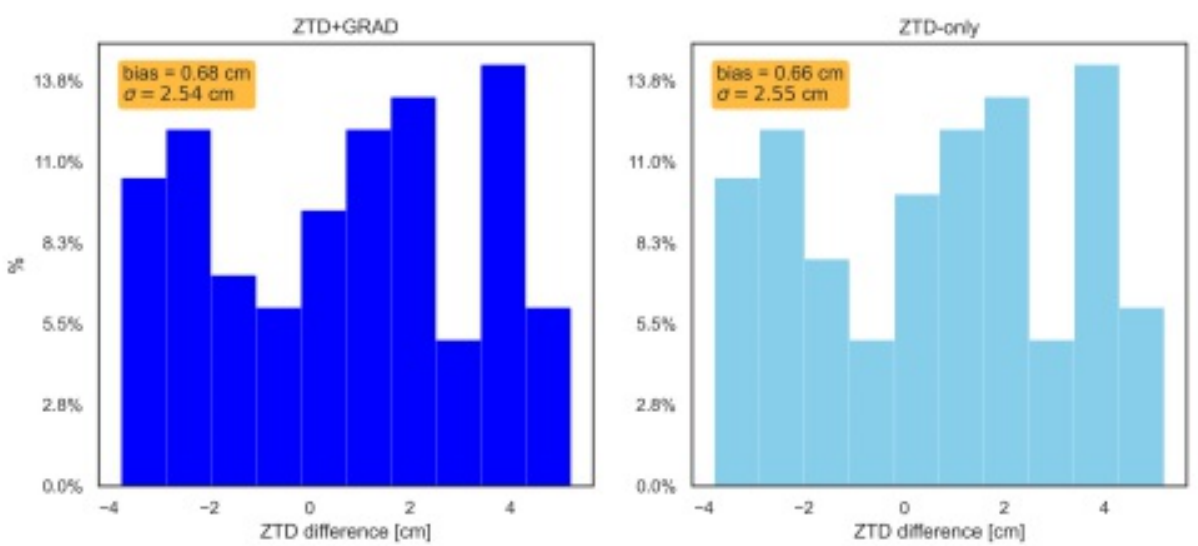

Figure 8.41: Histograms of GNSS-ZTD differences [cm] to ERA5-ZTD with/without gradient estimation: (1) ZTD+GRAD (dark blue),(2) ZTD-only (light blue). 


\subsubsection{Mis-modelling of ionosphere using SEID}

This section addresses the problem of a possible miss-modelling of the ionospheric delay when using the SEID model and its influence on the ZTD solution. Therefore a different section from the train track of CS1 was processed using (1) CSRS-PPP, (2) PPPW and additionally (3) SGAMP. The results are shown in Figure 8.42.

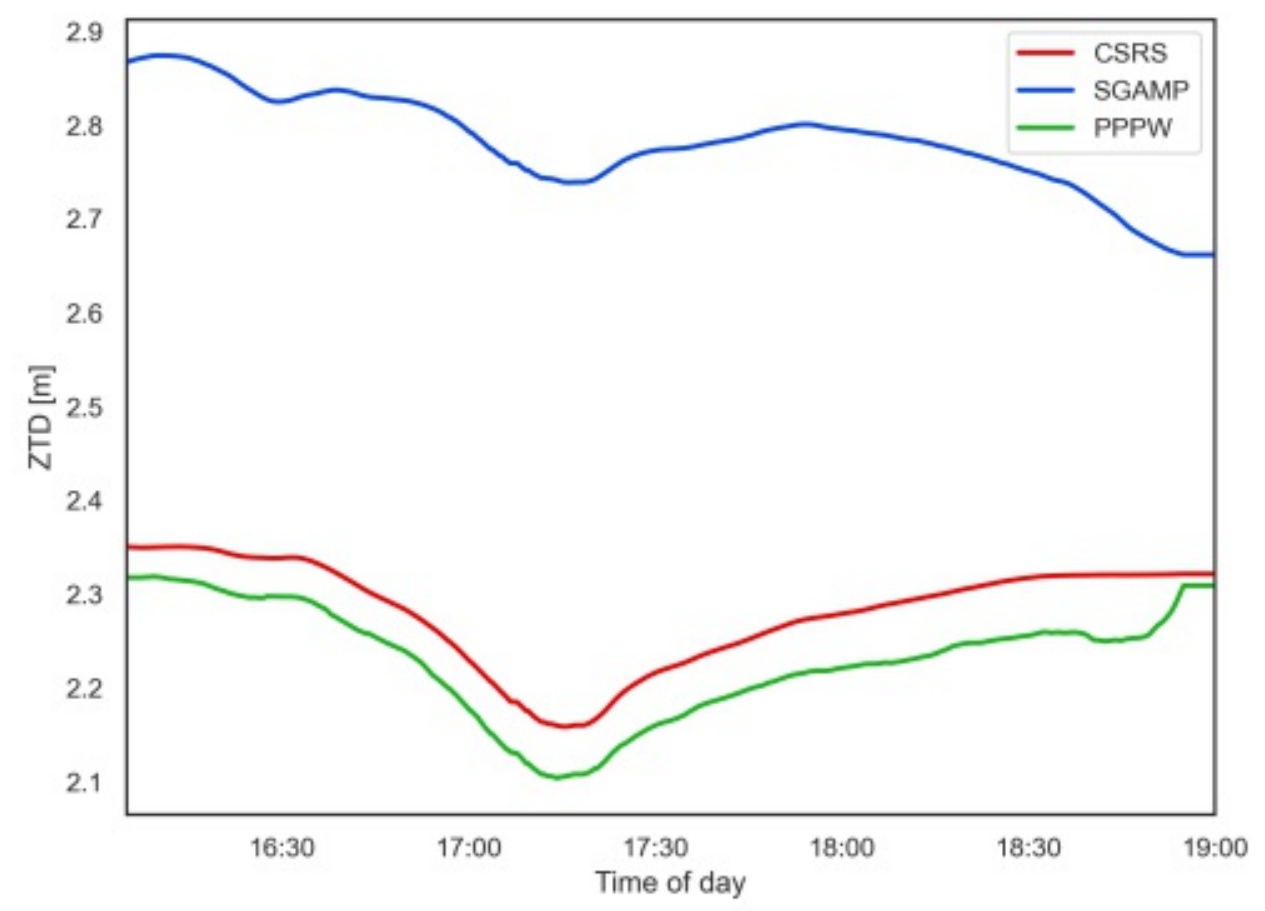

Figure 8.42: Comparison of GNSS-ZTD time series from CSRS-PPP (red), SGAMP (blue) and PPPW (green) for 16:00 to 19:00, 11.05.2017 (date of CS1).

A distinctive bias of about $5 \mathrm{dm}$ is visible for the SGAMP time series compared to the CSRS-PPP and PPPW results. However ERA5-ZTD was not specifically derived for this period, i.e. a full validation as for the case studies was not carried out. Nevertheless the large bias present indicates problems in the SEID model, whos usage represents the key difference to the other schemes. Since these have shown reasonable performance for all test cases and in particular also CS1, this cross-comparison seems sufficient to estimate the influence of miss-modelling the ionsopheric delay using SEID.

As shown in Section 6.4.1, the accuracy of the ionospheric modelling using SEID is strongly dependent on the quality of the phase observations (from both DF and SF stations) used. In case this quality is degraded by e.g. cycle slips, the model algorithm might not be able to mitigate these errors, especially in the case of large cycle slips which 
remain undetected.

This is represented in the large offset the SGAMP ZTD time series shows for this case. Even though the GAMP PPP engine tries to detect and correct present cycle slips in DF-PPP porcessing using the MW-LC, a large bias is still present in the ZTD results. Processing details from SGAMP reveal that for over $50 \%$ of epochs cycle slips have been detected. Many of those detected cycle slips show magnitudes from 10-50 m and where probably introduced by single, undetected cycle slips (in SF or DF data). In extreme cases like this one, they are able to propagate through the SEID algorithm or are even amplified by it.

This shows the immense importance of rigorous cycle slip detection and quality control for the retrieved GNSS raw data before using them in the SEID model. Cycle slips in DF data are generally easier to detect. This can either be done using common strategies as outlined in Section 6.2 or in the SEID algorithm by identifying abnormally large values of epoch-differenced GF-LCs (dL4), as shown in Figure 6.3. For SF data, the methods described in Section 6.2 have to be applied prior to usage of the data in SEID algorithm. A detection later in PPP processing using DF detection methods gets much more challenging since the observations on both carrier frequencies are not independent any more.

\subsubsection{Altitude-dependency of estimation errors}

As a last point of the sensitivity studies presented here, an assessment of the error behaviour with varying altitude is carried out. This denotes an interesting question which was already assessed by Webb et al. (2016) for a single railway track in North Wales. They found no obvious degradation with altitude for different estimation strategies.

For this study, four different test cases are available for CSRS-PPP and PPPW and can be averaged. Nevertheless one has to keep in mind that these tracks all can have substantially different features (multipath, shadowing, satellite geometry at travel time, ...), also at equal altitudes. Therefore this assessment can only serve as a first indication for the comparison of the solutions in this respect.

The results presented in Figures 8.43 and 8.44 represent differences between GNSS-ZTD (CSRS-PPP and PPPW) and ERA-ZTD for altitudes from 200 - 1000 meters (x-axis), averaged over the analysed railway tracks for different processing strategies.

Figure 8.43 presents the error evolution with height averaged over all four test cases/tracks. Overall results indicate better performance for PPPW at lower altitudes 
(up to 450-500 m). At higher altitudes the PPPW performance slightly decreases compared to CSRS-PPP, which shows better results from there on.

In general, performance increases with increasing altitude for both solutions. CSRS-PPP errors stay pretty constant at the $2-3 \mathrm{~cm}$ level for $500 \mathrm{~m}$ upwards. Noticeable is also a clear increase in performance for both GNSS-ZTD solutions around $400 \mathrm{~m}$. This might be due to statistical reasons (most of the covered tracks lie above these heights) or increased encountering of (more) challenging conditions at the lower altitudes (urban parts, multipath,...).

In addition, Figure 8.44 shows the results for each case separately, which enables to

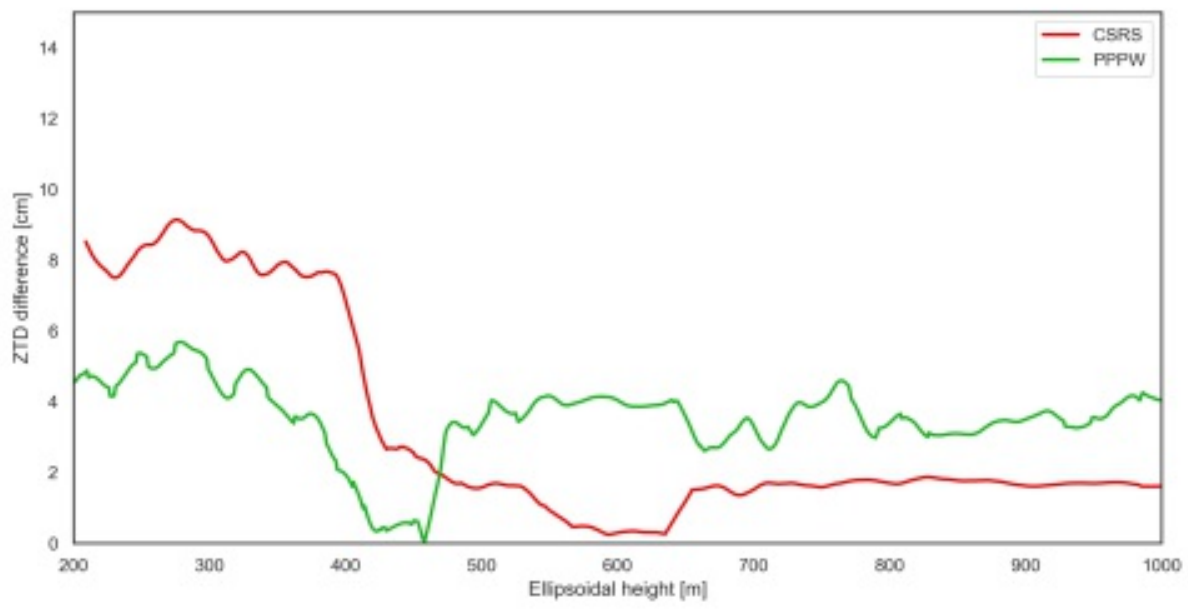

Figure 8.43: Absolute GNSS-ZTD differences [cm] with respect to ERA5-ZTD, averaged over all four test cases for CSRS-PPP (red) and PPPW (green) over the covered altitude range from 200 - 1000 m ellipsoidal height.

analyse each track/route and its characteristics separately. This is of interest since, as outlined in the case studies before, ZTD results can differ significantly from each other for the different tracks/dates.

CS2 (28.09.2017, top left) and CS3 (29.11.2019, bottom right) confirm the findings presented in the averaged solution above, i.e. better performance of CSRS-PPP at altitudes higher than $500 \mathrm{~m}$. Again the difference stays almost constant (especially visible for CS2) with increasing altitude.

CS1 (11.05.2017, top left) and especially CS4 (01.12.2019, bottom right) reveal different patterns in the comparison between CSRS-PPP and PPPW. PPPW outperforms CSRS-PPP over the first part of CS1. These results become clear when looking at the height distribution of the route which stays under $450 \mathrm{~m}$ most of the time (essentially altitudes where PPPW has lower average errors as well). 
Results for CS4 differ from those of the other test cases by showing better performance for PPPW with increasing altitude. Below approximately $700 \mathrm{~m}$ both solutions show almost equal performance, above PPPW biases are $\sim 1 \mathrm{~cm}$ lower than for CSRS-PPP. Reasons for this behaviour are challenging to find, maybe the fact that this case represents a night travel (and therefore different ionospheric conditions) plays a role.
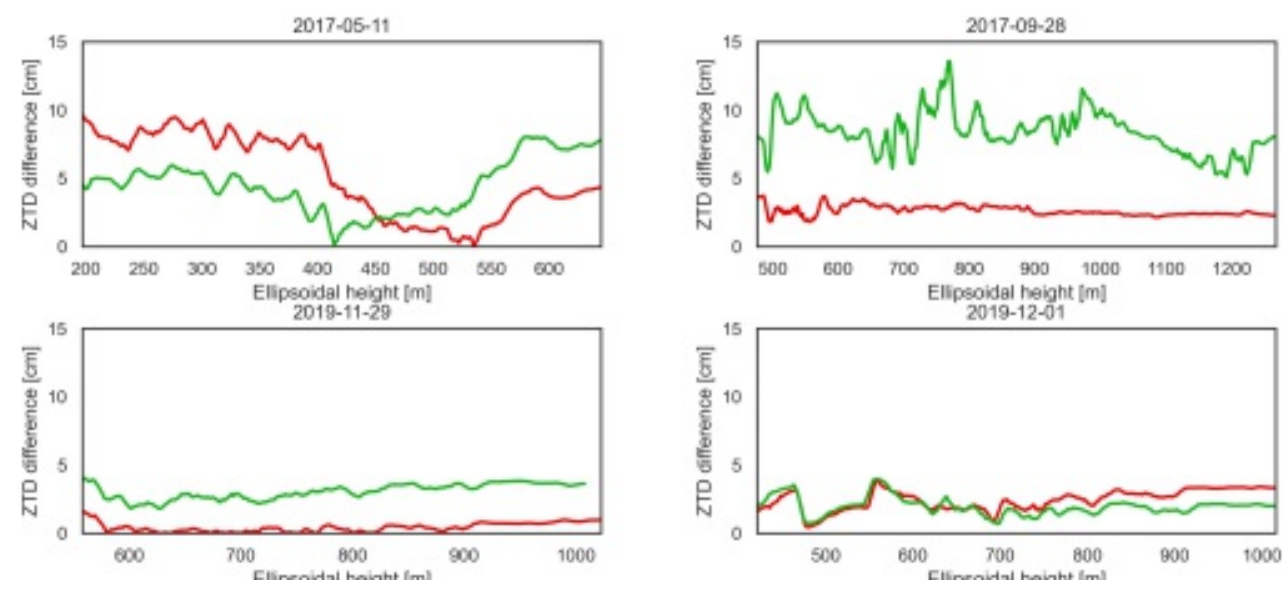

Figure 8.44: Absolute ZTD differences $[\mathrm{cm}]$ with respect to ERA5-ZTD, shown for all four test cases separately for CSRS-PPP (red) and PPPW (green) over the covered altitude range from 200 - 1000 m ellipsoidal height.

Overall the assessment of this section can be summarized by the following points:

- Overall performance slightly improves with increasing altitude.

- CSRS-PPP outperforms PPPW at altitudes above $450 \mathrm{~m}$, while for lower altitudes PPPW performs better.

- One test case (CS4), covering a night-time travel shows completely contrary results, with lower errors for PPPW at higher, and approximately equal performance of both solutions at lower altitudes. 


\subsection{Assimilation tests in WRF}

This last section in Chapter 8 provides the results of the assimilation tests using the WRF model. These tests should not be equated with full impact studies for ZTD assimilation as found in a lot of the literature presented in Section 7.3. The setup is rather reduced and concentrates fully on investigating the required quality of GNSS-ZTD to enter the DA system and if this quality can be achieved by the results presented in the last sections. Therefore a few considerations have to be noted here before results are presented:

- Due to both time and computational resources the results here focus on the actual DA procedure. This means that only the WRFDA module was run and an assessment of the quantity of GNSS-ZTD observations entering the procedure (and therefore the models initial state) is presented.

- No actual forecast was run due to reasons mentioned above and also due to the unlucky coincidence that almost no precipitation has been observed over Austria on the investigated days. This would make it hard to verify an (beneficial) impact on precipitation/moisture forecasts, even if the computational resources would have been available.

- Furthermore GNSS-ZTD data is only available from one railway track per analysed case study and therefore its impact on actual forecasts is assumed to be limited. However this will change as soon as data from multiple tracks can be obtained simultaneously.

- The assimilation runs of WRFDA were carried out by my colleague Natalia Hanna.

\subsubsection{WRF setup}

The WRF model domains utilized for the tests are shown in Figures 8.46 and 8.48. The parent domain with $30 \mathrm{~km}$ horizontal resolution, shown in black, basically covers Central Europe and the first nested domain, shown in blue, with $10 \mathrm{~km}$ resolution is fitted to the Austrian borders. Furthermore a third, very small domain with $3 \mathrm{~km}$ resolution was added in order to cope with the mountainous terrain that is covered by the train route. The general settings for the assimilation tests are summarized in Table 8.23 below.

\subsubsection{Influence of horizontal resolution}

The most important setting for the DA setup in conditions as present in this study (mountainous terrain + moderate/unknown quality of observation data) is the horizon- 


\begin{tabular}{lc} 
Parameter & Setting \\
\hline Periods & CS2/CS3 $(1 \mathrm{~h}$ each $)$ \\
Horizontal resolution (d01,d02,d03) & $30 / 10 / 3 \mathrm{~km}$ \\
Background data and boundary conditions & ERA5 \\
Observation interval (GNSS-ZTD) & $60 \mathrm{~s}$ \\
Assimilation method & $4 \mathrm{D}-\mathrm{Var}$ \\
Assimilation window & $1 \mathrm{~h}$ \\
Observation operator & GPSZTD \\
Observation error (pre-defined in WRFDA) & $0.5 \mathrm{~cm}$ \\
Error threshold & $2.5 \mathrm{~cm}$
\end{tabular}

Table 8.23: WRFDA settings chosen for the assimilation tests.

tal resolution of the model domain. It determines the quality of the background/model topography which is crucial for the calculation of ZTD values in the model domain (referred to as WRF-ZTD from now on). Since the quality check is based on the deviation between GNSS-ZTD and WRF-ZTD, the accuracy of WRF-ZTD directly influences the results of the check and therefore the decision which GNSS-ZTD observations are accepted by WRFDA.

Figure 8.45 displays the deviations between WRF-ZTD and GNSS-ZTD for two different setups: (1) using two model domains with 30/10 km horizontal resolution ( $\mathrm{d} 01, \mathrm{~d} 02)$, (2) using an additional third domain of $3 \mathrm{~km}$ resolution (d03), nested into the $10 \mathrm{~km}$ domain. In addition the error threshold for the quality check is also indicated.

Obviously, the GNSS-ZTD deviations with respect to WRF-ZTD are much too large in setup (1), whereas for setup (2) all observations are accepted by WRFDA (in case (2) only 10 points in the domain were used!). This behaviour becomes intuitive when one keeps in mind the environment in which the data was gathered. Even at the less problematic sections of the routes, e.g. broader valleys like the Inn valley, great changes in topography can be experienced within a few kilometres horizontal distance. In narrow alpine valley or at passes, altitude changes from 500-1000 m can be found on less than a kilometre horizontal distance, in extreme cases. If due to a poor horizontal model resolution e.g. a point on a mountain top is chosen instead of the respective point on the track, a huge error will be introduced in WRF-ZTD. Therefore it becomes clear that in such environments a horizontal resolution of $10 \mathrm{~km}$ will not be sufficient, a fact that has been a major problem in NWP for the last decades and was also experienced here within the first try-outs. 


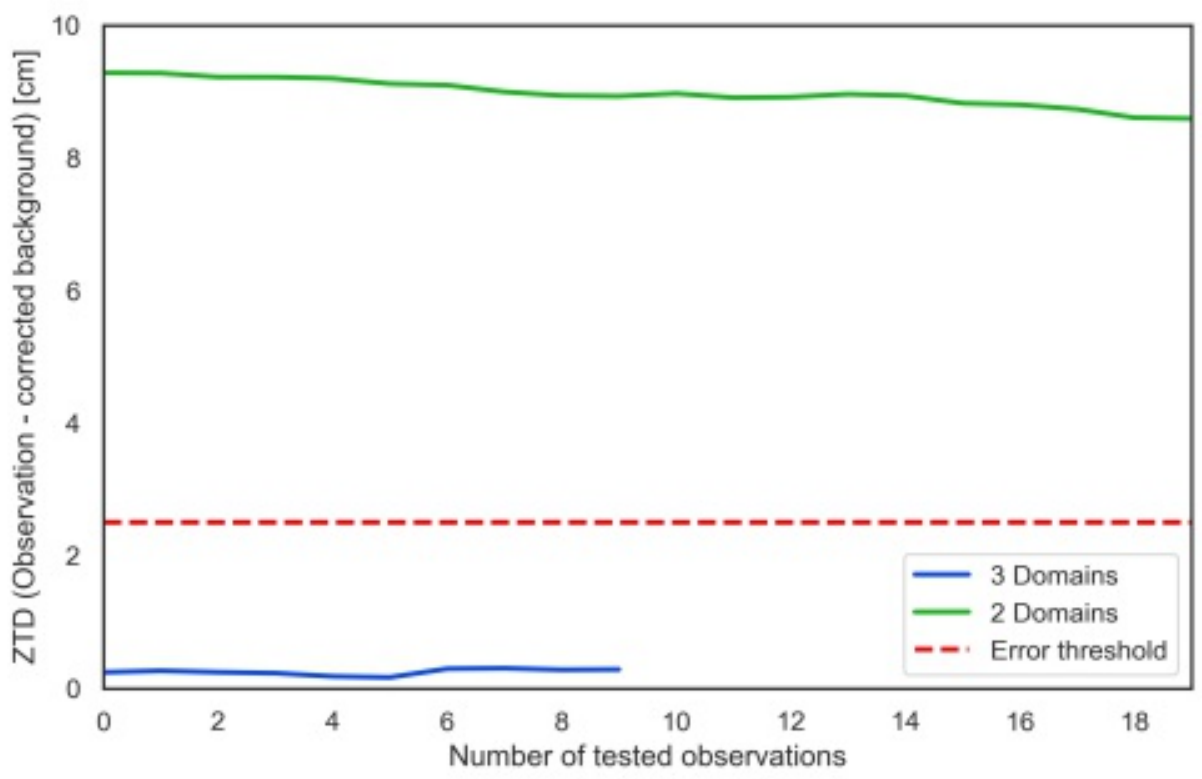

Figure 8.45: Comparison of numbers of assimilated observations for two different WRFDA setups: (1) using two model domains with $30 / 10 \mathrm{~km}$ horizontal resolution (green), (2) using an additional third domain of $3 \mathrm{~km}$ resolution (blue), nested into the $10 \mathrm{~km}$ domain. Additionally the error threshold for the quality check is also shown in red. 


\subsubsection{Assimilation test: CS3}

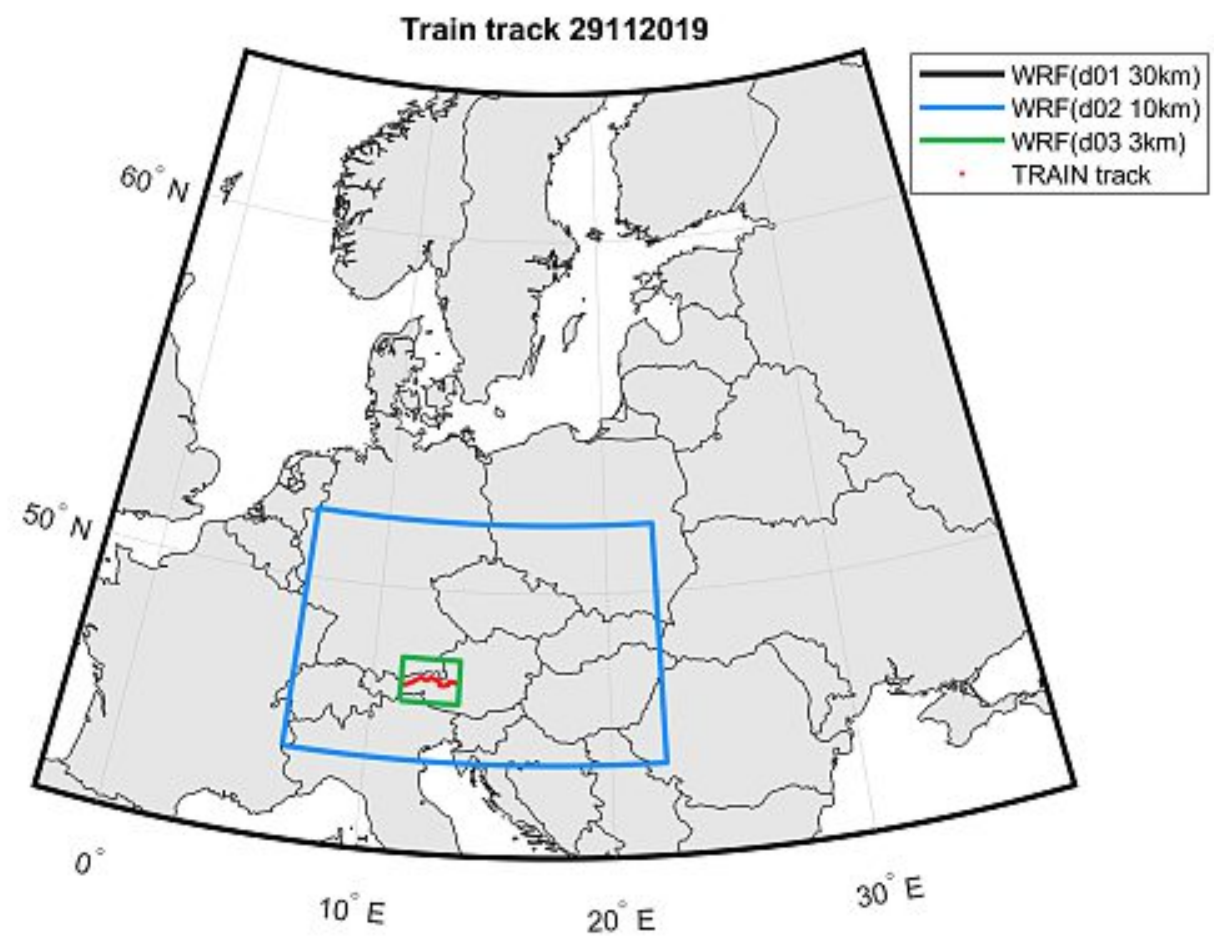

Figure 8.46: Plot of the used WRF model domains for CS3: parent domain (d01, black) and nested domains ( $\mathrm{d} 02$ and $\mathrm{d} 03$, blue and green).

The results of the tests for CS3 are presented in Figure 8.47. All important DA settings (for both test cases) were already outlined in Table 8.23.

In general, the CS3 test shows very promising results for both, the CSRS-PPP and the PPPW solution. All observations of CSRS-PPP got assimilated (60/60 points, right part) and show comparable or sometimes even smaller deviations to WRF-ZTD than to ERA-ZTD. The PPPW performance is also on a high level with $49 / 60$, i.e. $\sim 81$ $\%$ of observations entering WRFDA. These results show the feasibility of using such GNSS-ZTD estimates for DA and back up the results derived in the validation in the last sections. This manifests in the unexpectedly large number of accepted observations (given the challenging conditions and low-cost equipment) as well as in the fact that CSRS-PPP outperforms PPPW by a small margin.

\subsubsection{Assimilation test: CS2}

Although not chronologically, test case CS2 is analysed second here due to its more challenging conditions and therefore also significantly different results. Figure 8.48 shows 

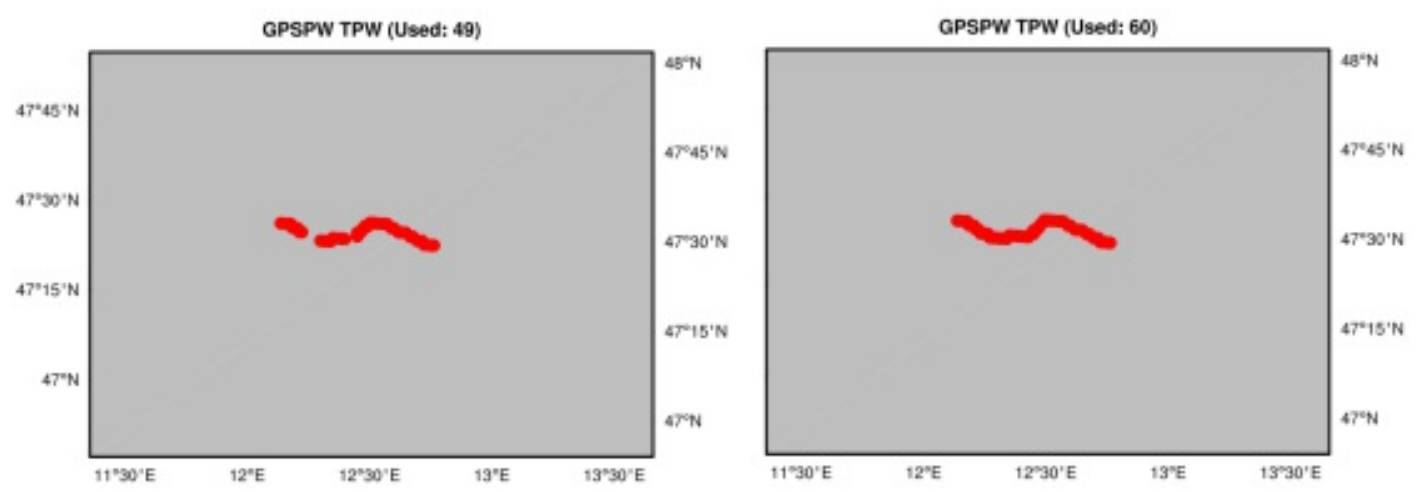

Figure 8.47: Observations assimilated for CS3 using WRFDA for different processing schemes. Processing schemes shown are: PPPW (left) and CSRS-PPP (right).

the three model domains $(30 / 10 / 3 \mathrm{~km})$ and the actual track passed by the train. Before discussing this test case in detail, it should be noted here that the chosen time slot for assimilation (from 09 - 10 UTC) corresponds to the most challenging part of the track, the actual passing of the alpine ridge (see plots shown in Section 8.3). This was chosen on purpose in order to investigate the DA procedure and observation usability in the most challenging conditions possible. The results of the tests for CS2 are presented

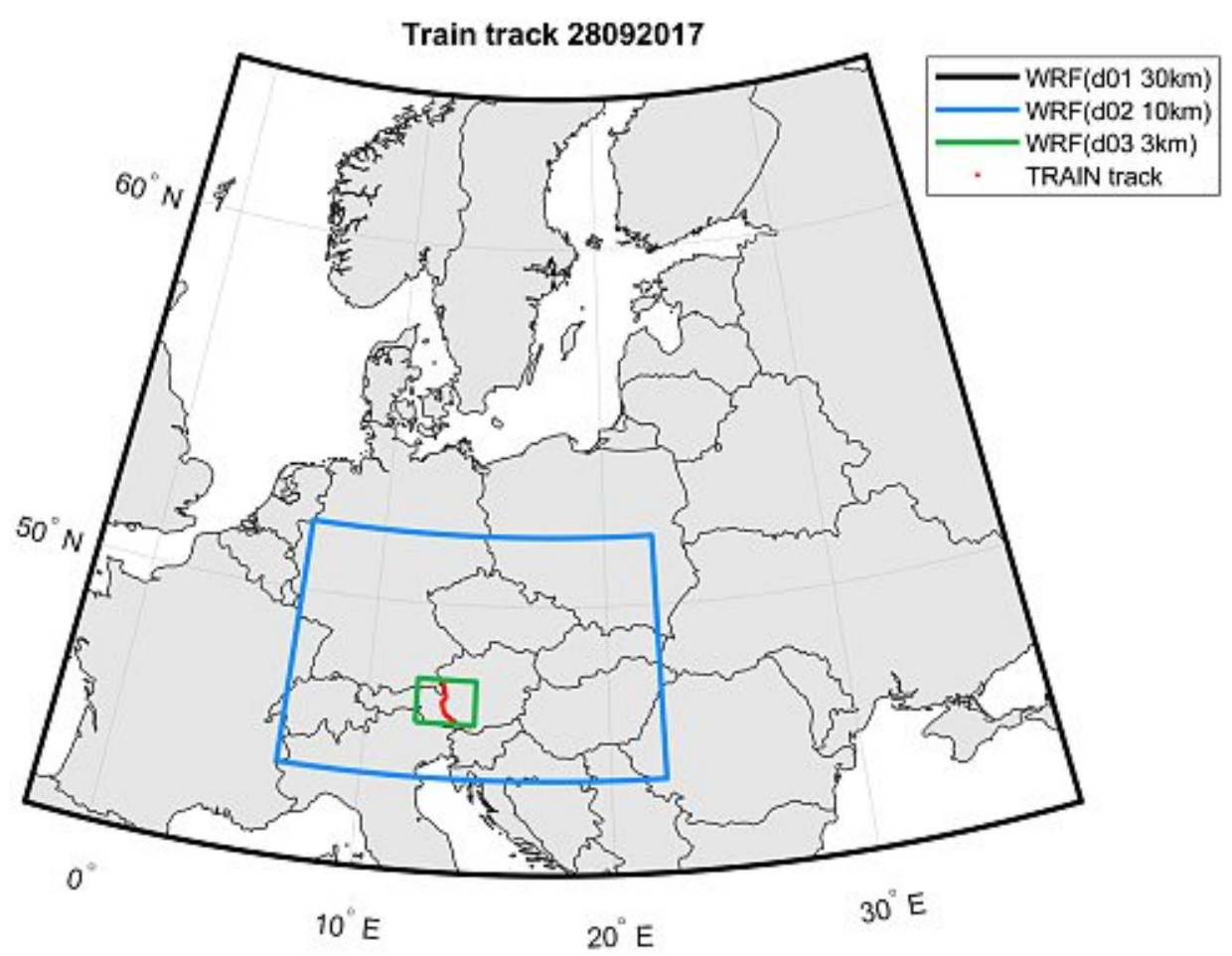

Figure 8.48: Plot of the used WRF model domains for CS3: parent domain (d01, black) and nested domains ( $\mathrm{d} 02$ and $\mathrm{d} 03$, blue and green). 
in Figure 8.49, in terms of the number of GNSS-ZTD observations which successfully entered the WRFDA system.

The top left track thereby represents the reference, showing all investigated observation points in the model (background state of the model, total of 56 points). The other three parts show results for SGAMP (top right), PPPW-HC (bottom left) and PPPW (bottom right). CSRS-PPP is left out of this comparison since surprisingly no observations of it passed the quality check of WRFDA. This behaviour seems anomalous when looking at results of the validation presented in the last sections and even more so if one looks to the first assimilation test, shown in the prior section.

The other three schemes also perform much worse than it could be expected when looking to the prior test case (CS3). Nevertheless for e.g. PPPW-HC still approximately half of the derived GNSS-ZTD got assimilated (29/56, 52\%). For SGAMP $21(37.5 \%)$ and for PPPW 14 (25\%) observations passed the quality check.

An interesting feature of those results is also the areal distribution of assimilated observations for each processing scheme. It shows some major differences in agreement with the model state/background forecast. SGAMP and PPPW-HC seem to fit better in the first part of the track (first half hour) whereas all accepted observations from PPPW were gathered at the end of the track.

The overall results of CS2 might represent what was to be expected from the data in the first place (prior to the start of this study) but they are somehow disturbing considering the results of CS3, especially for CSRS-PPP. Main suspicion is that environmental conditions (concerning GNSS and DA) are even more challenging for this case than for the others. The test case represents the passing of the main alpine ridge and therefore very mountainous terrain including heavily obstructed narrow valleys, tunnels and large horizontal changes of topography. All these features may play their part in the results shown here. Some further discussion on this topic will be addressed in Chapter 10 . 

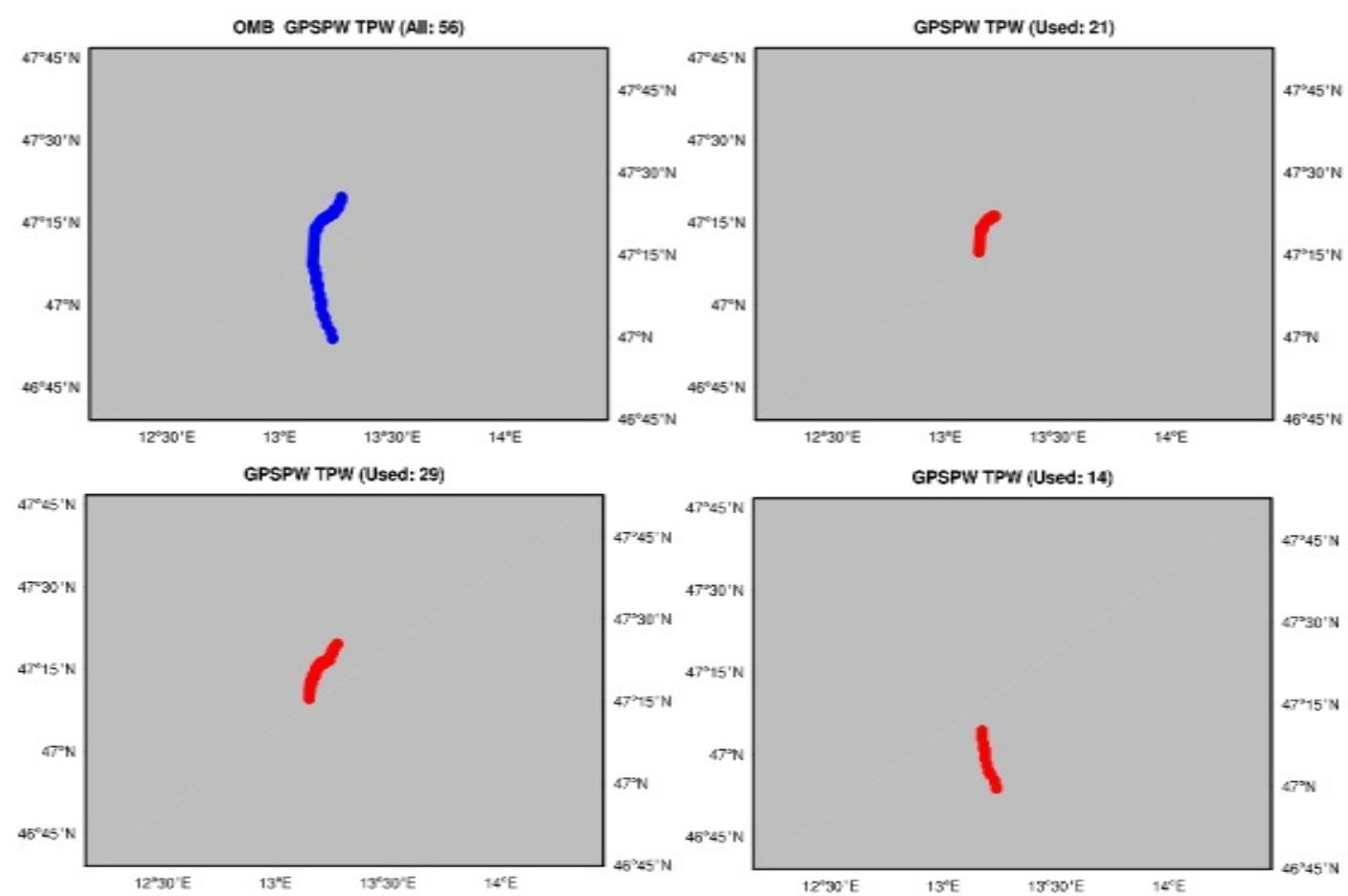

Figure 8.49: Observations assimilated using WRFDA for different processing schemes. Reference observations of model background are shown in blue, top left. Processing schemes shown are: SGAMP (top right), PPPW-HC (bottom left) and PPPW (bottom right). 


\section{Chapter 9}

\section{Towards real-time processing}

This last chapter aims to provide the basis for a future near real-time (NRT) application of the approach proposed in this thesis. Continuous provision of NRT tropospheric parameters from train-tracking data could represent a huge benefit for NWP, especially for very short-term weather forecasts (called Nowcasting) where observations have to be available in a very narrow time window (i.e. with the shortest latency time possible). A detailed discussion of these aspects is given in Section 9.3.

As first efforts were already started to be taken in form of a research project by the Research Division Higher Geodesy at TU Vienna, this chapter should serve as a basic guideline for changes to be implemented to the strategies given in this study.

Within the course of E-GVAP, NRT-ZTD estimations are already provided for a large number of stations (see Chapter 5). Even investigations on real-time retrieval of ZTD have been carried out, e.g. by Lu et al. (2017) who used IGS real-time product streams for PPP processing. Hadas et al. (2020) recently defined an advanced processing strategy, which reduced the a posteriori error of estimated ZTD (formal error) by $41 \%$ and deviations to final products from IGS by $17 \%$. Their overall suggestions are:

- Apply inter-system weighting for multi-GNSS observations

- Usage of an elevation-dependent cosine-type weighting function

- Estimate horizontal gradients

These studies could provide some basic strategies for NRT processing of Greenlight GNSS data on an operational basis. Nevertheless, refinements and adaptations accounting for the challenging environment and data quality issues have to be introduced. The following sections outline some important changes which have to be made to the described postprocessing approach conducted within this study, in order fit for NRT data processing. 


\subsection{Required adaptations for NRT GNSS processing}

In order to provide tropospheric products at the required time intervals for nowcasting applications some changes to the current implementation are necessary. This concerns a number of points of the whole approach which are detailed in the following:

\section{Data transmission}

\section{Orbit/clock products}

\section{Processing}

\section{Output interval}

\section{Data format results}

\subsubsection{Data transmission}

Since in this thesis a post-processing approach was chosen, data to be processed was available long before start of computation. In contrast, for NRT processing a regular data retrieval has to be set up close after tracking.

Solutions for this problem might be aided by the enhancement of telecommunication networks to $5 \mathrm{G}$ technology in order to secure fast data transfer from sensor to processing engine. Integration of cloud services for data storage and processing might also be feasible since data amounts will be significantly dependent on the number of utilized trains.

\subsubsection{Orbit/clock products}

Final orbit/clock solutions can no longer be used since they are not available on time for processing. RT streams (e.g. from IGS) or ultra-rapid orbit/clock products are a possible substitute for this problem.

Nevertheless, a degradation to a certain degree must be expected compared to the presented results.

\subsubsection{Processing software}

In the course of this work, interpreted programming languages where used for preprocessing (Matlab) and implementation of SEID (Python). Suggestion for a NRT application is to use compiled languages to maximise processing speed and therefore 
reduce latency time of the results.

Furthermore a number of different software packages (in different processing steps) were used in this study, which automatically leads to a lot of work due to data transfer between the software and processing steps. In the worst case, this may also lead to additional errors introduced in these transfer processes. In order to avoid these confusions it may be beneficial to implement a full processing chain in one single software package. This should include all steps necessary from data retrieval to delivery to potential end users. As already mentioned above, an implementation in a compiled language (e.g. $\mathrm{C}++, \mathrm{C}$, Fortran) is to be strived for. Another possibility beside designing an own, new package would be the extension of open-source packages to some of those used in this study (PPP-Wizard, GAMP) or the PPP software package raPPPd, developed at the research division Higher Geodesy at TU Vienna, which is also part of the Vienna VLBI and Satellite Software (VieVS) (Böhm et al. 2018).

\subsubsection{Output interval}

This represents a key point concerning the usage of the produced datasets in DA setups for NWP. A well-defined balance has to be found between different aspects like:

- Use as much valuable data as possible for DA

- Investigate how much more data still represents a benefit for forecasts/verification results

- Investigate how much data can be gathered/processed/assimilated without running into computational (resource) problems

All these aspects have to be considered when defining an optimal output interval for the ZTD data. This is clearly to be done in close cooperation with end users of the data (NWP users/operators like meteorological institutes). Therefore, a definite recommendation is beyond the scope of this thesis.

Nevertheless, the investigated interval of one minute ZTD output, as introduced in Section 7.4 should be mentioned here again. Its performance was reasonable for an investigation of pre-study status like this one here. As already mentioned before, a lot of further research might be necessary on this, in order to derive the best possible choice for an operational implementation. 


\subsubsection{Data format of NRT products}

Although this might be a minor point, the format in which results are provided to possible users should still be discussed here. Currently the only existing standard format for GNSSderived tropospheric products is the COST-Format (E-GVAP 2014). All processing products established at the Research Division Higher Geodesy at TU Vienna are delivered in this format.

However due the special nature of the data (much higher time-resolution possible, but only from one receiver/train per file) one might think about alternatives. A highly standardized and widely-used format, especially for meteorological applications, is the NETCDF format. This one could easily be adopted for the needs of this application and most meteorological models are familiar with it. Nevertheless it might also be possible to redefine the COST-format based on the structure of the train-borne ZTD estimates.

\subsection{Purposed workflow for NRT processing}

In general, two options for NRT PPP processing of Greenlight GNSS data are discussed in the following. They are closely related to the approaches outlined in this study for post-processing, with the relevant changes described before.

- SF-PPP using regional ionosphere maps

- DF-PPP using SEID model

\subsubsection{SF-PPP using regional ionosphere maps}

This can be referred to as the standard approach when working with SF GNSS observations. Therefore it was also used for most processing carried out in this study and its performance is well documented for different software packages in Chapter 8.

Nowadays a number of different models are available for both global and regional ionosphere modelling. Most of these provide corrections in form of VTEC maps with about \pm 1 -2 TEC units accuracy, which can be mapped to the elevation of every satellite in view. Using these STEC values, a ionospheric range correction can be applied.

\subsubsection{DF-PPP using SEID model}

The alternative approach to the usage of TEC maps presented above is a "synthetic upgrade" to DF observations using the SEID approach (Deng et al. 2009). The SEID model was already introduced in detail before (Section 6.4), thus this section focuses 
on the implementation of a processing strategy comprising the SEID algorithm it as a prerequisite. A possible approach for implementation is shown using the flowchart in Figure 9.1.

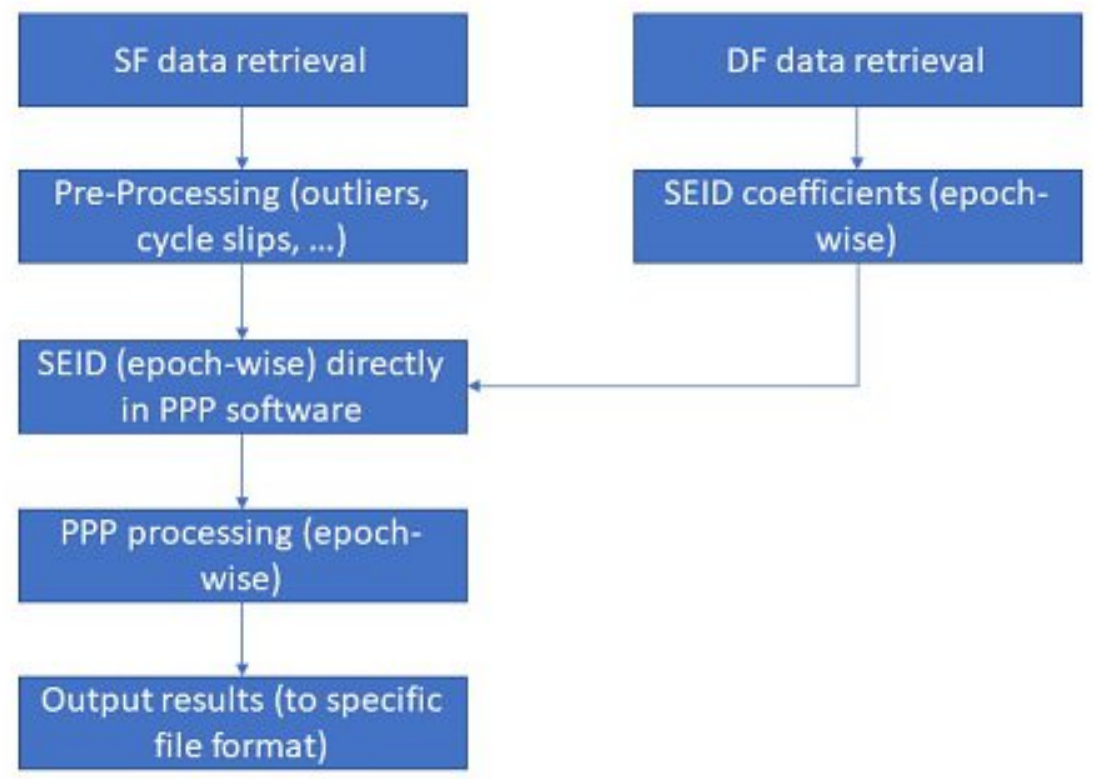

Figure 9.1: Possible Flowchart for SEID DF-PPP processing in a NRT-implementation of ZTD estimation from Greenlight GNSS data.

1. In the first step the SF data from the train as well as DF reference station data have to be gathered. Ideally this process is carried out simultaneously, using the principles of Parallel I/O.

2. Then both datasets are pre-processed, containing steps introduced in Section 6.2, in order to secure only data of usable quality enters the SEID model. Again it would be favourable to parallelize this step for SF/DF data.

3. In the third step common satellites of SF and DF dataset are determined and the interpolation coefficients of the SEID model can be calculated from the DF data.

4. These coefficients are applied to the SF data in form of the SEID model, generating a second frequency observation. 
5. This extended dataset is processed in PPP mode using the IF-LC in an epochwise manner. In the case that continuous data streams of SF/DF data can be established, the whole process could be repeated epoch-wise, avoiding large I/O of RINEX data in the process.

\subsection{Requirements for NWP assimilation and applica- tions}

NRT products (from GNSS or other sensors) are of immense importance for NWP applications, especially very short-term weather forecasting (Nowcasting) and severe weather warning systems. Nowcasting products for instance need to be produced rapidly (ideally available to customers within 15 minutes of data time) (Ballard et al. 2016). Moreover it is also crucial to match the observations as closely as possible in the first hours of the forecast. A number of studies have outlined the benefits of NRT tropospheric parameters from GNSS for nowcasting over the last decade. An extended overview of those investigations was already given in Chapter 7, Section 7.3.

As already mentioned the 4D-Var assimilation shall be utilized to exploit the high temporal resolution of the GNSS-ZTD estimates in an optimal way. The next sections describe a feasible example setup to realize an operational mode of Greenlight-GNSS-ZTD assimilation for operational nowcasting.

\subsubsection{DA configuration}

The configuration of the DA system is a key point for ensuring the greatest possible benefit of the GNSS-ZTD estimates. In this study, usage of the 4D-Var technique was purposed. A number of studies have already investigated the potential of this technique for short-term weather forecasts and nowcasting (Ballard et al. (2016), Thiruvengadam et al. (2020), Li et al. (2018), ...). It was shown by Li et al. (2018) that 4D-Var assimilation has a positive impact on precipitation forecasting skills compared to the corresponding 3D-Var assimilation for the whole nowcast period (six hours from analysis time) and forecasts for the United Kingdom clearly benefited from the use of extra, higher time frequency observations in both 3D-Var and 4D-Var setups.

An example 4D-Var setup proposed by Ballard et al. (2016) is shown in Figure 9.2. This setup was used within the Nowcasting Demonstration Project (NDP) for precipitation forecasts during the London Olympics 2012.

A key factor of the implementation, which is also shown in Figure 9.2, is the observa- 


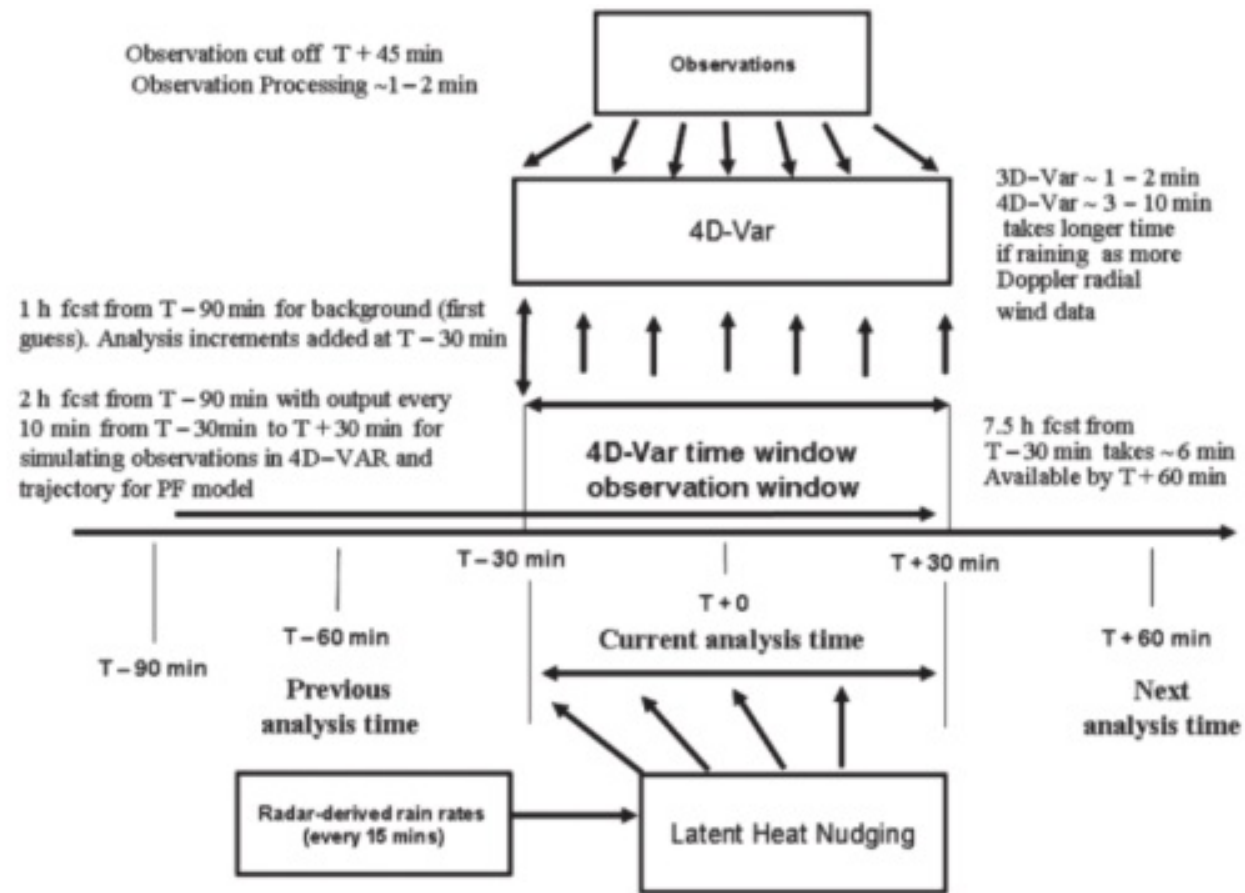

Figure 9.2: Exemplary DA configuration using 4D-Var technique. Taken from Ballard et al. (2016)

tion window, i.e. the time window from which observations can be used to determine the next analysis. The study suggests that hourly GNSS-ZTD data from reference stations typically arrived too late for the shown setup, which uses an observation window of 60 minutes. This is a familiar problem which the Research Division Higher Geodesy at TU Vienna frequently encountered in the production of NRT estimates for ZAMG from an Austrian reference station network (Weber et al. 2019). Such a drawback could be resolved by the Greenlight ZTD estimates which are available on a shorter time-scale (e.g. 15 minutes as proposed before). Therefore a similar setup like shown above could be used in case of using 4D-Var for assimilation of the ZTD estimates.

However a refined strategy definition for this problem is far beyond the scope of this thesis. Therefore this proposed suggestion should only serve as a reference point for possible future implementations. It depends largely on the actual NWM and its compatibility with such a DA system and thus has to be coordinated with the model operators. 


\subsection{Extension to advanced tropospheric parameters}

In terms of data delivery, the current setup could be extended for advanced tropospheric products, i.e slant delays and horizontal gradients. Estimation of horizontal gradients was already investigated in the course of this thesis, see Section 8.6. Gradient estimation has shown to be beneficial for GNSS-ZTD quality (Zus et al. (2019a), Hadas et al. $(2020), \ldots)$ as well as for variational DA methods (Zus et al. (2019b)). However, in this study only the influence on ZTD estimation was analysed, not the quality of the actual gradient estimations themselves. From the results shown in Section 8.6, no beneficial effect could be found on ZTD solutions when extending the parameter estimation for gradients. This disagrees with literature available up till now on this topic (Zus et al. (2019a), Hadas et al. (2020)). Nevertheless, one has to keep in mind that these studies focused on static measurements, mostly from high-quality data gathered at reference stations placed strategically. However, the initial situation for the data used in this study is significantly different. This might concern the following considerations:

1. In challenging GNSS environments, like frequently encountered in the railway domain, satellite visibility and therefore geometry often is degraded. Therefore redundancy is not always sufficient to provide high-quality estimates or even provide a solution at all. As typically the position solution is the one of most interest, other parameters are often downgraded in the processing.

2. In the case of such highly kinematic observations it might be questionable if gradient parameters are as interesting for meteorological applications as in the static case. This addresses the fact that the sensor is moving relatively to a certain atmospheric phenomena to be observed. As much as these relative changes could be of interest, it could also introduce some interferences between these effects which might cancel out information which would be present in gradients of static station data.

Therefore reliable predictions on the benefit of train-borne atmospheric gradients for NWP can not be provided here, i.e. more efforts have to be put in such investigations in order to guarantee useful results.

From a technical point of view the extension to gradients is not too much effort since many software packages already include the possibility of gradient estimation. This can then easily be extended for STD estimates, using ZTD and gradient estimations to derive slant delays in a straightforward way, e.g. by implementation the formulation of Chen and Herring (1997) as realized in the software ATom (Möller 2017). 


\section{Chapter 10}

\section{Conclusions and outlook}

This study assessed the feasibility of deriving tropospheric parameters from low-cost GNSS receivers operating on trains and their usability for assimilation in NWP models. Overall goal was a prove of concept for a full processing and analysis chain from data retrieval to processing, filtering and finally usage in a NWM.

The data utilized in this study originate from Greenlight, a project led by the Austrian Federal Railways, and were collected on a number of trains operating on different tracks all over Austria. Raw data obtained from the trains was processed and analysed in terms of position and especially tropospheric parameters, in detail ellipsoidal height and most importantly ZTD.

First key points in the data processing chain were a suitable pre-processing strategy which includes a multipath analysis and cycle slip detection.

Furthermore the fact that only SF observations are available from Greenlight necessitates a separate correction of the ionospheric influence. Therefore two different approaches were tested:

- Range correction using TEC maps derived from global ionosphere models

- Generation of DF data using the SEID model

Both approaches were found suitable to account for ionospheric delays up to the accuracy required for ZTD estimation.

All data were processed using a kinematic PPP approach deriving position and ZTD estimates from the tracking data. Although this common general approach is taken for all test cases, different strategies for certain processing aspects have been tested and the difference in performance was assessed. 
The utilized software packages were CSRS-PPP, PPP-Wizard and GAMP. All of them were able to provide reasonable height trajectories and ZTD estimates for all test cases.

In general, the results of the case studies (Test cases CS1,CS3,CS4) were analysed in terms of three basic aspects:

1. Raw data quality and environmental conditions

2. Analysis of parameter noise level of the PPP solutions for ZTD and height coordinate

\section{ZTD validation using ERA5-ZTD}

The first point included an analysis of the multipath level (using CMC observables) as well as geometry indicators in form of GDOP values. As expected, results show increased multipath levels (up to 100-150 metres) and degraded geometry (GDOP > 4-5), especially for track sections in urban and mountainous areas.

In the second step, the parameter noise of original PPP time series of ZTD and height coordinate was assessed by comparison to smoothed time series of the respective parameters. This comparison was carried out between the CSRS-PPP and the PPPW solution. This smoothed solution was produced using a MA-filter with a time window of five minutes.

The results indicate a very low noise level for both ZTD and height coordinate. Average bias values are in the sub millimetre-range for ZTD and in the millimetre-range for the height coordinate, indicating the suitability of the chosen time window of five minutes for the MA-filter. Standard deviation and RMS are in centimetre- (ZTD) and metre-range (height coordinate) and almost equal to each other for all test cases.

CSRS-PPP shows significantly lower noise levels (by a factor 10 on average) compared to PPPW for both ZTD and height and all case studies.

Finally, results from four different processing schemes (based on the three software packages mentioned above) have been validated using independent ZTD data derived from ERA5 reanalysis data:

- CSRS-PPP

- PPP-Wizard (PPPW)

- PPP-Wizard with height constraint (PPPW-HC) 
- GAMP using SEID model (SGAMP)

Statistical analysis of those results revealed good to excellent agreement with ERA-ZTD depending on the specific test case for both processing schemes. The solutions agree at the low $\mathrm{cm}$ - to $\mathrm{mm}$-level with ZTD derived from ERA5. On average, a significant superiority of one solution has not been recognized.

Although only part of the assessment for test case CS2, the SGAMP approach showed very promising results for this case. Results have a significantly lower bias $(1.34 \mathrm{~cm})$ compared to CSRS-PPP/PPPW $(2.4 / 8.8 \mathrm{~cm})$ and the correlation with ERA-ZTD is just as high as for the other solutions. This makes it a promising candidate for future investigations on this topic, although further experience and tests are definitely required. Furthermore the accuracy of GNSS-ZTD can be improved by applying a tight constraint on the height coordinate solution. This was achieved using height coordinates from the official track database of ÖBB and an implementation in the Kalman filter process of the PPPW software. Solutions show medium to large improvements compared to the standard PPPW version and the other solutions. With even smaller errors than SGAMP, this approach is definitely suitable for further research and test processing with larger datasets.

In a last validation step, certain processing options were tested in terms of sensitivity and performance of the ZTD solution. The conducted tests accounted for:

- Benefit of GLONASS observations: Very little to zero impact has been found due to the inclusion of GLONASS observations. This might indicate questionable quality of the observations. Exceptions are highly obstructed areas where they help to still provide a solution.

- Estimation of tropospheric gradients: Again no visible impact is present for the conducted tests. Although possible benefits are documented in literature. They might not be present in the investigated cases due to the challenging geometry, observation quality and low visibility of satellites in general for certain areas.

- Impact of elevation cut-off: Using only elevations equal or higher than $7.5^{\circ}$ shows clear benefits for ZTD accuracy compared to an inclusion of observations down to $5^{\circ}$. These findings somehow contradict with the fact that tropospheric estimates can be decorrelated from the height coordinate more efficiently when using a lower elevation cut-off. Nevertheless the results presented within this 
study are assumed to be heavily affected by the specific environments the data is collected in. High multipath influence on signals at low elevations and a limited number of those observations due to shading effects might be the main reasons behind the improvements from higher cut-off settings.

- Mis-modelling of ionospheric delay using SEID: The results show the huge sensitivity of the SEID model and its results to outliers and cycle slips in the utilized phase observations. If a large number of cycle slips remains undetected in pre-processing, errors introduced in the ZTD solution can reach decimetre-levels. Rigorous quality control and efficient cycle slip detection methods are of immense importance when using the SEID model for ionospheric mitigation.

- Altitude-sensitivity of ZTD errors: The analysis of the results shows a generally decreasing error in ZTD with altitude for both investigated schemes (CSRS-PPP and PPPW). This might be attached to the fact that with increasing altitude the amount of water vapour and therefore ZWD decreases. This means the relative impact of ZHD modelling on ZTD results is increased and this parameter is much easier to model than ZWD. On the other side, under normal circumstances mountainous terrain is more likely encountered at higher altitudes and therefore geometry and satellite availability suffer as well as multipath influence is present more often. On average, the first of those two considerations seems to be the more prominent one for this study.

Comparing the solutions from the schemes yields an advantage for PPPW at altitudes below $450 \mathrm{~m}$ and vice versa for CSRS-PPP above this altitude.

Furthermore, first tests for assimilation of GNSS ZTD data derived from trains were conducted for two of the test cases. Therefore the WRF model was utilized and a 4D-Var assimilation scheme for one hour of GNSS-ZTD (with resolution of one minute, i.e. 60 observations each case) was tested. Results are very promising in general, but with distinctive differences between the test cases. These most likely depend on the complexity and challenges of the GNSS environment (mountainous terrain, GNSS-denied areas like tunnels, urban areas).

For test case CS3, almost all GNSS-ZTD observations (CSRS-PPP: 100\%, PPPW: $81 \%$ ) passed the quality check of the WRFDA module, which performs the assimilation procedure within the WRF.

Test case CS2 however shows significantly worse results as for most of the four tested processing schemes not even half of the observations were accepted. This likely reflects the fact that this case presents the most challenging route to be analysed 
within this study. An interesting behaviour also observed here are local differences between processing solution against WRF-ZTDs. For three out of four schemes (CSRSPPP,PPPW-HC and SGAMP) only observations in the first part of the investigated track were assimilated, for PPPW only observations from the end of the track. The turning point seemed to be the actual alpine passing (points of highest altitude) where tunnels were encountered. It could be guessed that a bias introduced after this tunnel passage causes this distinctive behaviour but further research and case studies are definitely needed to solve this issue, and for DA of those GNSS-ZTD in general.

However these tests did not include actually running a (precipitation) forecast, due to, amongst other things, limited (computational) resources. Therefore this investigation can only serve as a first indication of the usability of the GNSS-ZTD estimates in a NWM.

Finally, the feasibility of the presented approaches for operational NRT processing of GNSS tracking data has been analysed and some necessary adaptations to the presented processing schemes were outlined. A number of challenges will arise from the extension of the presented post-processing study to an operational mode and an extensive test period is required to back up the findings of this thesis.

Nevertheless, this extension is possible and will be of great benefit for other scientific studies and even operational usage. Also positioning applications for train localisation systems like developed within Greenlight will benefit from improved processing strategies and troposphere modelling.

First and foremost, the idea of having a large fleet of trains (up to hundreds of vehicles) and using each one of them as a meteorological sensor for a parameter as important as water vapour will definitely be of huge interest for atmospheric sciences. The horizontal and temporal resolution which can be provided is not achieved by any other sensor at the moment. Moreover, a first indication that the results are of sufficient quality was already given in this study. This quality will also increase in future mainly due to better hardware and more sophisticated processing approaches.

The most important benefits will hopefully arise for the production of more accurate weather forecasts used for nowcasting, severe weather warnings and a number of other NWP products. 


\section{Acronyms}

ABL Atmospheric Boundary Layer

APC Antenna Phase Center

AR Ambiguity Resolution

ARP Antenna Reference Point

ARAMIS Advanced Railway Automation Management Information System

AROME Application of Research to Operations at MEsoscale

AURIS Automatisches Reisenden-Informationssystem

BOC Binary Offset Carrier

CMC Code-Minus-Carrier

CNES Centre National d'Etudes Spatiales

COSMO Consortium for Small-scale Modeling

CS Case Study

CSRS Canadian Spatial Reference System

DA Data Assimilation

DD Double-Difference

DF Dual-Frequency

DOD Department of Defence

DOP Dilution of Precision

EC European Commission 
ECMWF European Center for Medium-Range Weather Forecast

EGNOS European Geostationary Navigation Overlay Service

EKF Extended Kalman Filter

EPOSA Echzeit Positionierung Austria

ERA ECMWF Re-Analysis

ESA European Space Agency

FIR Finite Impulse Response

GFZ GeoForschungsZentrum

GIM Global lonosphere Map

GMF Global Mapping Function

GNSS Global Navigation Satellite Systems

GLONASS Globalnaya navigatsionnaya sputnikovaya sistema

GPS Global Positioning System

GSA European GNSS Agency

GSM Global System for Mobile

HAS High-Accuracy Service

IF-LC lonosphere-Free Linear Combination

IFS Integrated Forecasting System

IGS International GNSS Service

I/O Input/Output

IPP lonospheric Pierce Point

ITRF International Terrestrial Reference Frame

IWV Integrated Water Vapour

LC Linear Combination 
LEO Low Earth Orbiting

LMMSE Linear Minimum Mean Square Error

LSQ Least Squares

MA Moving Average

MSE Mean Square Error

MMSE Minimum Mean Square Error

MPM Millimeter-wave Propagation Model

NCEP National Centers for Environmental Prediction

NRT Near-Real Time

NWM Numerical Weather Model

NWP Numerical Weather Prediction

OEBB Oesterreichische Bundesbahn

OTL Ocean Tidal Loading

PPP Precise Point Positioning

PWV Precipitable Water Vapour

QPF Quantitative Precipitation Forecasting

RINEX Receiver Independent Exchange Format

RO Radio Occultation

RT Real Time

RTK Real Time Kinematic

RUC Rapid Update Cycle

SA Selective Availability

SAR Search-and-Rescue

SBAS Satellite-based Augmentation System 
SEID Satellite-specific Epoch-differenced lonospheric Delay model

SF Single-Frequency

STEC Slant Total Electron Content

TEC Total Electron Content

TEPOS T-Kom Services Echtzeit-Positionierung

VieVS Vienna VLBI and Satellite Software

VTEC Vertical Total Electron Content

VMF Vienna Mapping Function

WGS-84 World Geodetic System 84

ZHD Zenith Hydrostatic Delay

ZTD Zenith Total Delay

ZWD Zenith Wet Delay 


\section{List of Figures}

2.1 Geometric concept of GNSS positioning, shown here in the 2D case. Taken from Böhringer (2008) . . . . . . . . . . . . . . . . . 9

2.2 Component and error sources in pseudorange measurements, taken from Sanz Subirana et al. (2013) . . . . . . . . . . . . . . . . . . . . . 16

3.1 A vertical column of air of density $\rho$, horizontal cross-sectional area $\delta \mathrm{A}$, height $\delta \mathrm{z}$ and mass $\mathrm{M}=\rho \delta \mathrm{A} \delta \mathrm{z}$. The pressure on the lower surface is $\mathrm{p}$, the pressure on the upper surface is $\mathrm{p}+\delta \mathrm{p} . \ldots 26$

3.2 Typical atmospheric temperature profile from U.S. standard atmosphere, taken from Wallace and Hobbs (2006) . . . . . . . . . . . . . . 28

3.3 Climatological average precipitation rate $(\mathrm{mm} /$ day) for $1981-2010$ from ERA-Interim, Global Precipitation Climatology Centre(GPCC), Global Precipitation Climatology Project (GPCP) and E-OBS (European Observations) taken from https://climate.copernicus.eu/ monthly-summaries-precipitation-relative-humidity-and-soil-moisture 33

4.1 lonospheric layers present at day- and nighttime and their respective heights, taken from https://commons. wikimedia.org/w/index.php?curid=7009115 . . . . . . 37

4.2 Ionospheric electron density profiles computed from the International Reference lonosphere (IRI) model for Vienna in 2017, taken from Magnet (2019) . . . . . . . . . . . . . . . . . . . . . . 38

4.3 VTEC map for 26.02.2010, 12 UTC derived using the Klobuchar model. Typical maximums in VTEC are visible at low latitudes. Taken from Najman and Kos (2014)

4.4 Height profiles of the hydrostatic $\left(N_{h}\right)$ and wet component $\left(N_{w}\right)$ of the refractivity $N$ for two different days, originating from the same time of the day and station. $N_{h}$ shows a continuous curve, $N_{w}$ high variability in time and space. Taken from Nilsson et al. (2013). . . . . . . . . . . . . 41 
4.5 Path of a GNSS signal trough the troposphere. The actual path $\mathrm{S}$ is longer than the geometric path $\mathrm{G}$ due to the bending effect and arrives at a different elevation $e_{0}$. Taken from Nilsson et al. (2013) . . . . . . . . 41

5.1 Basic illustration of the GNSS Meteorology concept. Individual STDs are mapped to ZTD for a single station. Taken from Guerova et al. (2016). . 46

5.2 Timeline of European projects in the field of GNSS Meteorology from 1996 - 2016. Taken from Guerova et al. (2016) . . . . . . . . . . . . . 47

5.3 Principle of GNSS tomography, taken from Dong and Jin (2018) . . . . 49

5.4 Basic principle of GNSS RO, Source: ECMWF . . . . . . . . . . . . . . 50

6.1 Overview of systems working together in Greenlight, courtesty of DI (FH) Manfred Stättner, ÖBB Infrastrucktur . . . . . . . . . . . . . . . . . 54

6.2 Reference station network of EPOSA. The colour of the triangles denotes the company operating the station. Taken from http://www.eposa.at/ . 55

$6.3 \delta L_{4}$ values [m] for an example epoch and all GPS satellites processed within the SEID model. Larger values indicate possible outliers or cycle slips in the data of the respective satellite (e.g. G32 here). . . . . . . . . 64

6.4 Flowchart of the SGAMP processing scheme as followed in this study. . . 69

7.1 ERA5 global grid of 2m-temperature for January 2016. Top: full reanalysis, bottom: ERA5-Land reanalysis. Taken from: https://climate.copernicus.eu/climate-reanalysis . . . . . . . . . . . . 79

7.2 The basic principle of DA and its main components outlined for the temperature ( $T, y$-axis) parameter and a 24-hour assimilation window (x-axis): background forecast (blue), observations (green) and analysis (red).

7.3 Simplified schematic representations of four data assimilation strategies frequently-used in NWP:(a) 3D-Var,(b) 3D-FGAT,(c) incremental 4DVar, and (d) EnKF. Blue circles represent observations, red lines represent model trajectories, and purple diamonds indicate analyses. The dotted red lines in(b)represent linearly interpolated or extrapolated forecast values used to estimate increments at observation times. The dashed red lines in(c)represent the initial forecast, prior to iterative adjustments. These illustrations are conceptual and do not accurately reflect the much more complex strategies used by reanalysis systems. Taken from Fujiwara et al. (2017) . . . . . . . . . . . . . . . . . . . . . . 82 
7.4 System components of Advanced Research WRF, taken from Barker et al. (2012).

8.1 Train-track covered for CS1 (Salzburg - Vienna airport) derived from CSRS-PPP processing. . . . . . . . . . . . . . . . . . . . . . . . . 92

8.2 Code-Minus-Carrier (CMC) observables for CS1, shown are all visible GPS satellites.

8.3 Number of visible satellites (left) and GDOP values (right) along the CS1 route.

8.4 CS1 PPP ZTD time series from CSRS-PPP (top) and PPPW (bottom). The raw PPP ZTD estimates are shown in red, the MA-smoothed (Interval $5 \mathrm{~min}$ ) in blue.

8.5 CS1 PPP ellipsoidal height time series from CSRS-PPP (top) and PPPW (bottom). The raw PPP height estimates are shown in red, the MAsmoothed (Interval $5 \mathrm{~min}$ ) in blue.

8.6 Comparison between estimated GNSS-ZTD (CSRS-PPP (red) and PPPW (green)) and ERA5-ZTD (black) for CS1 (11.05.2017), Top: absolute values, bottom: ZTD differences between GNSS-ZTD with respect to ERA5-ZTD

8.7 Histograms of ZTD differences between GNSS-ZTD and ERA5-ZTD for test case CS1. Left: CSRS, Right: PPPW. Also indicated are bias and standard deviation $(\sigma)$ of the respective solutions.

8.8 Correlation between ZTD as estimated by each software package and the ERA5 reference values for CS1, 11.05.2017: (left) CSRS-PPP and (right) PPPW. Terms $\mathrm{R}$ and $\mathrm{y}$ represent the correlation coefficient and the linear fit, respectively.

8.9 Correlation between ZTD as estimated by each software package and the ERA5 reference values for CS1, excluding the first hour of data (12:0013:30): (left) CSRS-PPP and (right) PPPW . Terms $\mathrm{R}$ and y represent the correlation coefficient and the linear fit, respectively. . . . . . . . . . 99

8.10 Train-track covered for CS2 (Munich - Klagenfurt shown here) derived from CSRS-PPP processing. . . . . . . . . . . . . . . . . . 100

8.11 CMC observables for CS2, shown are all visible GPS satellites. . . . . . . 101

8.12 Number of visible satellites (left) and GDOP values (right) along the CS2 route. . . . . . . . . . . . . . . . . . . 102 
8.13 CS2 PPP ZTD time series from CSRS-PPP (top) and PPPW (bottom). The raw PPP ZTD estimates are shown in red, the MA-smoothed (Interval $5 \mathrm{~min}$ ) in blue. . . . . . . . . . . . . . . . . . . . 103

8.14 CS2 PPP ellipsoidal height time series from CSRS-PPP (top) and PPPW (bottom). The raw PPP height estimates are shown in red, the MAsmoothed (Interval $5 \mathrm{~min}$ ) in blue. . . . . . . . . . . . . . 103

8.15 Train-track covered for CS2 with the reference stations utilized for SEID processing shown in blue dots. . . . . . . . . . . . . . . . . . 105

8.16 Comparison between estimated GNSS-ZTD (CSRS-PPP (red), SGAMP (blue) and PPPW (green) and PPPW-HC (lime)) and ERA5-ZTD (black) for CS2 (28.09.2017), Top: ZTD, bottom: differences between GNSSZTD with respect to ERA5-ZTD . . . . . . . . . . . . . . . 106

8.17 Histograms of ZTD differences between GNSS-ZTD and ERA5-ZTD for test case CS2. Top left: CSRS-PPP, Top right: SGAMP, Bottom left: PPPW, Bottom right: PPPW-HC. Also indicated are bias and standard deviation $(\sigma)$ of the respective solutions. . . . . . . . . . . . 108

8.18 Correlation between ZTD as estimated by each software package and the ERA5 reference values for CS2, 28.09.2017. Top left: CSRS-PPP, Top right: SGAMP, Bottom left: PPPW, Bottom right: PPPW-HC. Terms $\mathrm{R}$ and $\mathrm{y}$ represent the correlation coefficient and the linear fit, respectively. 109

8.19 Train track of CS3 (29.11.2019): Innsbruck - Woergl - Bischofshofen Liezen . . . . . . . . . . . . . . . . . . . . . 110

8.20 CMC observables for CS3, shown are all visible GPS satellites. . . . . . . 111

8.21 Number of visible satellites (left) and GDOP values (right) along the CS3 route. . . . . . . . . . . . . . . . . . . 111

8.22 CS3 PPP processing results for ZTD from CSRS-PPP (top) and PPPW (bottom). The actual PPP output is shown in red, the MA-smoothed (Interval $5 \mathrm{~min}$ ) in blue.

8.23 CS3 PPP ellipsoidal height time series from CSRS-PPP (top) and PPPW (bottom). The raw PPP output is shown in red, the MA-smoothed (Interval $5 \mathrm{~min}$ ) in blue.

8.24 Comparison between estimated GNSS-ZTD (CSRS-PPP (red) and PPPW (green)) and ERA5-ZTD (black) for CS3 (29.11.2019), Top: ZTD, bottom: differences between GNSS-ZTD with respect to ERA5-ZTD114 
8.25 Histograms of ZTD differences between GNSS-ZTD and ERA5-ZTD for test case CS3. Left: CSRS, Right: PPPW. Also indicated are bias and standard deviation $(\sigma)$ of the respective solutions.

8.26 Correlation between GNSS-ZTD as estimated by each software package and the ERA5 reference values for CS3, 29.11.2019: (left) CSRS-PPP, (middle) PPPW and (right) PPPW-HC. Terms $\mathrm{R}$ and y represent the correlation coefficient and the linear fit, respectively. . . . . . . . . . . 116

8.27 Train track of CS4 (01.12.2019): Innsbruck - Woergl - Bischofshofen Liezen - Leoben - Bruck/Mur - Graz . . . . . . . . . . . . . . . . 117

8.28 CMC observables for CS4, shown are all visible GPS satellites.

8.29 Number of visible satellites (left) and GDOP values (right) along the CS3 route. . . . . . . . . . . . . . . . . . . . . 118

8.30 CS4 PPP ZTD time series from CSRS-PPP (top) and PPPW (bottom). The raw PPP output is shown in red, the MA-smoothed (Interval 5 min) in blue.

8.31 CS4 PPP ellipsoidal height time series from CSRS-PPP (top) and PPPW (bottom). The raw PPP output is shown in red, the MA-smoothed (Interval $5 \mathrm{~min}$ ) in blue.

8.32 Comparison of ellipsoidal height between PPP and RTK solutions for CS4 (01.12.2019): CSRS-PPP (red) / PPP-Wizard (green) / RTK solution (black). Original processing output time series shown on top, MA-filtered time series at the bottom.

8.33 Comparison between estimated GNSS-ZTD (CSRS-PPP (red) and PPPWizard (green)) and ERA5-ZTD (black) for CS4 (01.12.2019), Top: absolute values, bottom: differences between GNSS-ZTD with respect to ERA5-ZTD . . . . . . . . . . . . . . . . . . . . . . . . . . 122

8.34 Histograms of ZTD differences between GNSS-ZTD and ERA5-ZTD for test case CS4. Left: CSRS, Right: PPP-Wizard. Also indicated are bias and standard deviation $(\sigma)$ of the respective solutions.

8.35 Correlation between ZTD as estimated by each software package and the ERA5 reference values for CS4, 01.12.2019: (left) CSRS-PPP, (middle) PPPW and (right) PPPW-HC. Terms R and y represent the correlation coefficient and the linear fit, respectively. 
8.36 Comparison of GNSS-ZTD with ERA5-ZTD with/without GLONASS observations for CS3: (1) GPS+GLONASS (dark green),(2) GPS-only (light green). Absolute ZTD values (top) and absolute differences to ERA5ZTD (bottom).

8.37 Comparison of GNSS-ZTD with ERA5-ZTD with/without GLONASS observations for CS4: (1) GPS+GLONASS (dark green),(2) GPS-only (light green). ZTD values (top) and differences to ERA5-ZTD (bottom). . . . 126

8.38 Comparison of GNSS-ZTD with ERA5-ZTD for different settings for elevation cut-off: (1) $5^{\circ}$ (dark blue),(2) $10^{\circ}$ (light blue). Absolute ZTD values (top) and absolute differences to ERA5-ZTD (bottom) . . . . . . . 128

8.39 Histograms of GNSS-ZTD differences to ERA5-ZTD for different settings for elevation cut-off: (1) $5^{\circ}$ (dark blue),(2) 7.5/10 (light blue) . . . . . 128

8.40 Comparison of GNSS-ZTD with ERA5-ZTD with/without gradient estimation: (1) ZTD+GRAD (dark blue),(2) ZTD-only (light blue). Absolute ZTD values (top) and absolute differences to ERA5-ZTD (bottom). 130

8.41 Histograms of GNSS-ZTD differences [cm] to ERA5-ZTD with/without gradient estimation: (1) ZTD+GRAD (dark blue),(2) ZTD-only (light blue). . . . . . . . . . . . . . . . . . . . . . . 130

8.42 Comparison of GNSS-ZTD time series from CSRS-PPP (red), SGAMP (blue) and PPPW (green) for 16:00 to 19:00, 11.05.2017 (date of CS1). 131

8.43 Absolute GNSS-ZTD differences [cm] with respect to ERA5-ZTD, averaged over all four test cases for CSRS-PPP (red) and PPPW (green) over the covered altitude range from 200 - 1000 m ellipsoidal height. . . 133

8.44 Absolute ZTD differences [cm] with respect to ERA5-ZTD, shown for all four test cases separately for CSRS-PPP (red) and PPPW (green) over the covered altitude range from $200-1000$ m ellipsoidal height. . . . . . 134

8.45 Comparison of numbers of assimilated observations for two different WRFDA setups: (1) using two model domains with 30/10 km horizontal resolution (green), (2) using an additional third domain of $3 \mathrm{~km}$ resolution (blue), nested into the $10 \mathrm{~km}$ domain. Additionally the error threshold for the quality check is also shown in red. . . . . . . . . . . . . . . . 137

8.46 Plot of the used WRF model domains for CS3: parent domain (d01, black) and nested domains ( $\mathrm{d} 02$ and d03, blue and green) . . . . . . . . 138

8.47 Observations assimilated for CS3 using WRFDA for different processing schemes. Processing schemes shown are: PPPW (left) and CSRS-PPP (right). 
8.48 Plot of the used WRF model domains for CS3: parent domain (d01, black) and nested domains ( $\mathrm{d} 02$ and d03, blue and green) . . . . . . . . 139

8.49 Observations assimilated using WRFDA for different processing schemes. Reference observations of model background are shown in blue, top left. Processing schemes shown are: SGAMP (top right), PPPW-HC (bottom left) and PPPW (bottom right). . . . . . . . . . . . . . . . . . . . . 141

9.1 Possible Flowchart for SEID DF-PPP processing in a NRTimplementation of ZTD estimation from Greenlight GNSS data. . . . . . 147

9.2 Exemplary DA configuration using 4D-Var technique. Taken from Ballard et al. (2016) . . . . . . . . . . . . . . . . . . . . . . . 149 


\section{List of Tables}

2.1 Components and frequencies for civil use of GPS signal . . . . . . . . 10

2.2 Defining parameters of WGS-84, taken from Hofmann-Wellenhof et al. (2012) . . . . . . . . . . . . . . . . . . . . . . . 12

2.3 Components and frequencies of Galileo signal . . . . . . . . . . . . . . 14

2.4 Overview on noise and multipath characteristics of GNSS observables, most information taken from Möller (2017). . . . . . . . . . . . . . . . 21

3.1 Net radiation budget of the Earth, Möller (2017) . . . . . . . . . . . . . 24

3.2 Fractional concentrations of gaseous atmospheric constituents with respect to dry air, with important greenhouse gases marked bold. Taken from Wallace and Hobbs (2006) . . . . . . . . . . . . . . 25

5.1 Overview of assimilation strategies used for GNSS-derived tropospheric products in popular NWP models: WRF (Weather Research and Forecasting), COSMO (Consortium for Small-scale Modeling), AROME (Application of Research to Operations at MEsoscale) and IFS (Integrated Forecasting System, operated at ECMWF) model . . . . . . . . . . . . . 48

6.1 Overview on methods for cycle slip detection (Farooq et al. 2018). . . . 58

6.2 Default settings and features for the CSRS-PPP package. . . . . . . . . 67

6.3 Default settings and features for the PPP-Wizard software. . . . . . . . 68

6.4 Default settings and features for the GAMP PPP software. . . . . . . . . 70

7.1 Detailed information on the product line of reanalyses produced by ECMWF. 76

8.1 Overview of conducted case studies . . . . . . . . . . . . . . . . . 89

8.2 General PPP settings chosen for the test cases. . . . . . . . . . . . . . . 91

8.3 Performance metrics for the PPP noise analysis of CS1 for CSRS-PPP and PPPW. Measures are computed for ZTD differences between original PPP output and smoothed solution. 
8.4 Performance metrics for the PPP noise analysis of CS1 for CSRS-PPP and PPPW. Measures are computed for height coordinate differences between original PPP output and smoothed solution. . . . . . . . . . . . 95

8.5 CS1: Performance metrics for the ZTD validation against ERA-ZTD for processing results of CSRS-PPP and PPPW . . . . . . . . . . . . . . . . . 98

8.6 CS1 (excluding 12:30 -13:30): Performance metrics for the ZTD validation against ERA-ZTD for processing results of CSRS-PPP and PPPW. . 99

8.7 Performance metrics for the PPP noise analysis of CS2 for CSRS-PPP and PPPW. Measures are computed for ZTD differences between original PPP output and smoothed solution. . . . . . . . . . . . . . . . . . . . 104

8.8 Performance metrics for the PPP noise analysis of CS2 for CSRS-PPP and PPPW. Measures are computed for height coordinate differences between original PPP output and smoothed solution. . . . . . . . . . . . 104

8.9 Reference stations chosen for SEID processing for CS2 . . . . . . . . . 104

8.10 CS2: Performance metrics for the ZTD validation against ERA-ZTD for processing results of CSRS-PPP, SGAMP and PPPW and PPPW-HC. . . 107

8.11 Performance metrics for the PPP noise analysis of CS3 for CSRS-PPP and PPPW. Measures are computed for ZTD differences between original PPP output and smoothed solution.

8.12 Performance metrics for the PPP noise analysis of CS3 for CSRS-PPP and PPPW. Measures are computed for height coordinate differences between original PPP output and smoothed solution. . . . . . . . . . . . 113

8.13 CS3: Performance metrics for the ZTD validation against ERA5-ZTD for processing results of CSRS-PPP, PPPW and PPPW-HC . . . . . . . . 116

8.14 Performance metrics for the PPP noise analysis of CS4 for CSRS-PPP and PPPW. Measures are computed for ZTD differences between original PPP output and smoothed solution. . . . . . . . . . . . . . . . . . . 118

8.15 Performance metrics for the PPP noise analysis of CS4 for CSRS-PPP and PPPW. Measures are computed for height coordinate differences between original PPP output and smoothed solution. . . . . . . . . . . . 119

8.16 Performance metrics for the PPP noise analysis of CS4 for CSRS-PPP and PPPW. Measures are computed for height coordinate differences between raw PPP output and the raw RTK solution. . . . . . . . . . . . 121

8.17 Performance metrics for the PPP noise analysis of CS4 for CSRS-PPP and PPPW. Measures are computed for height coordinate differences between smoothed PPP and RTK time series. 
8.18 CS4: Performance metrics for the ZTD validation against ERA5-ZTD for processing results of CSRS-PPP, PPPW and PPPW-HC . . . . . . . . 123

8.19 CS3: Performance metrics for the ZTD validation against ERA5-ZTD for processing results for GPS-only and GPS+GLONASS . . . . . . . . . . 125

8.20 CS4: Performance metrics for the ZTD validation against ERA5-ZTD for processing results for GPS-only and GPS+GLONASS . . . . . . . . . . 126

8.21 Performance metrics for the ZTD validation against ERA5-ZTD for processing results for $5^{\circ}$ and $7.5 / 10^{\circ}$ cut-off elevation angle settings. Investigated test case is CS2 . . . . . . . . . . . . . . . . . . . . 127

8.22 Performance metrics for the ZTD validation against ERA5-ZTD for processing results for ZTD-only and ZTD+GRAD settings. Investigated test case is CS2 . . . . . . . . . . . . . . . . . . . . . . . . . . . . 129

8.23 WRFDA settings chosen for the assimilation tests. . . . . . . . . . . 136 


\section{Bibliography}

Adavi, Z., W. Rohm, and R. Weber, 2020: Analyzing different parameterization methods in gnss tomography using the cost benchmark dataset. IEEE Journal Of Selected Topics In Applied Earth Observations And Remote Sensing, 13, 6155-6163, doi:10. 1109/JSTARS.2020.3027909.

Aichinger-Rosenberger, M., 2018: Usability of high-resolution GNSS-ZTD data in the AROME model. M.S. thesis, University of Innsbruck, URL https://diglib.uibk. ac.at/urn:nbn:at:at-ubi:1-27392.

Aichinger-Rosenberger, M. and R. Weber, 2020: Tropospheric delay parameters derived from GNSS-tracking data of a fast moving fleet of trains. 7th International colloquium on scientific and fundamental aspects of GNSS, 1.

Alkan, R. M., M. H. Saka, . M. Ozulu, and V. İlçi, 2017: Kinematic precise point positioning using GPS and GLONASS measurements in marine environments. Measurement, 109, 36 - 43, doi:https://doi.org/10.1016/j.measurement.2017.05.054, URL http://www.sciencedirect.com/science/article/pii/S0263224117303421.

Anthes, R. A., 2011: Exploring Earth's atmosphere with radio occultation: contributions to weather, climate and space weather. Atmospheric Measurement Techniques, 4 (6), 1077-1103, doi:10.5194/amt-4-1077-2011, URL https://amt. copernicus . org/articles/4/1077/2011/.

Ballard, S. P., Z. Li, D. Simonin, and J.-F. Caron, 2016: Performance of 4D-Var NWPbased nowcasting of precipitation at the Met Office for summer 2012. Quarterly Journal of the Royal Meteorological Society, 142 (694), 472-487, doi:10.1002/qj.2665, URL https://rmets.onlinelibrary.wiley.com/doi/abs/10.1002/qj . 2665.

Barker, D., et al., 2012: The weather research and forecasting model's community variational/ensemble data assimilation system: Wrfda. Bulletin of The American Meteorological Society - BULL AMER METEOROL SOC, 93, 831-843, doi: 10.1175/BAMS-D-11-00167.1. 
Beutler, G., I. Bauersima, S. Botton, W. Gurtner, M. Rothacher, and T. Schildknecht, 1989: Accuracy and biases in the geodetic application of the global positioning system. manuscripta geodaetica, 14 (1), 28-35.

Bevis, M., S. Businger, T. A. Herring, R. A. Anthes, and R. H. Ware, 1992: GPS Meteorology: Remote Sensing of Atmospheric Water Vapor Using the Global Positioning System. Geophys. Mag., 34, 359-425.

Böhm, J., et al., 2018: Vienna VLBI and satellite software (VieVS) for geodesy and astrometry. Publications of the Astronomical Society of the Pacific, 130 (986), 044503 , doi:10.1088/1538-3873/aaa22b.

Bisnath, S. and R. Langley, 2001: Pseudorange Multipath Mitigation By Means of Multipath Monitoring and De-Weighting.

Boehm, J., A. Niell, P. Tregoning, and H. Schuh, 2006: Global mapping function (gmf): A new empirical mapping function based on numerical weather model data. Schuh Geophys. Res. Lett, 25, doi:10.1029/2005GL025546.

Böhm, J., 2004: Troposphärische laufzeitverzögerungen in der vlbi. Ph.D. thesis, Geodäsie und Geophysik.

Böhm, J. and H. Schuh, 2004: Vienna Mapping Functions in VLBI analyses. Geophysical Research Letters, 31 (1029/2003GL018984).

Böhm, J., B. Werl, and H. Schuh, 2006: Troposphere mapping functions for GPS and very long baseline interferometry from European Centre for Medium-Range Weather Forecasts operational analysis data. J. Geophys. Res, 111.

Böhringer, F., 2008: Gleisselektive ortung von schienenfahrzeugen mit bordautonomer sensorik. Ph.D. thesis, doi:10.5445/KSP/1000007317.

Boisits, J., M. Glaner, and R. Weber, 2020: Regiomontan: A regional high precision ionosphere delay model and its application in precise point positioning. Sensors, 20 , 2845, doi:10.3390/s20102845.

Boniface, K., C. Champollion, J. Chery, V. Ducrocq, C. Rocken, E. Doerflinger, and P. Collard, 2012: Potential of shipborne GPS atmospheric delay data for prediction of Mediterranean intense weather events. Atmospheric Science Letters, 13 (4), 250-256, doi:10.1002/asl.391. 
Brenot, H., et al., 2013: Preliminary signs of the initiation of deep convection by GNSS. Atmos. Chem. Phys., 13, 5425--5449.

Chadwell, C. D. and Y. Bock, 2001: Direct estimation of absolute precipitable water in oceanic regions by GPS tracking of a coastal buoy. Geophysical Research Letters, 28 (19), 3701-3704, doi:10.1029/2001GL013280.

Chao, C., 1974: The troposphere calibration model for Mariner Mars 1971. JPL Technical Report, 32-1587.

Chen, G. and T. A. Herring, 1997: Effects of atmospheric azimuthal asymmetry on the analysis of space geodetic data. Journal of Geophysical Research: Solid Earth, 102 (B9), 20 489-20 502, doi:10.1029/97JB01739, URL https : //agupubs . onlinelibrary.wiley.com/doi/abs/10.1029/97JB01739.

Davis, J., T. Herring, I. Shapiro, A. Rogers, and G. Elgered, 1985: Geodesy by Radio Interferometry: Effects of Atmospheric Modeling Errors on Estimates of Baseline Length. Radio Science, 20, 1593-1607.

de Bakker, P. and C. Tiberius, 2017: Real-time multi-GNSS singlefrequency precise point positioning. GPS Solutions, 21, 1791-1803, doi: 10.1007/s10291-017-0653-2, https://agupubs.onlinelibrary.wiley.com/ doi/pdf/10.1029/2019EA000650.

de Haan, S., 2008: Meteorological applications of a surface network of Global Positioning System receivers. Ph.D. thesis, Wageningen University.

Deng, Z., M. Bender, G. Dick, M. Ge, and J. Wickert, 2009: Retrieving tropospheric delays from GPS networks densified with single frequency receivers. Geophysical Research Letters, 36, 36, doi:10.1029/2009GL040018.

DoD, 2020: Global positioning system standard positioning service performance standard. Assistant secretary of defense for command, control, communications, and intelligence.

Dodson, A., W. Chen, N. Penna, and H. Baker, 2001: GPS estimation of atmospheric water vapour from a moving platform. Journal of Atmospheric and Solar-Terrestrial Physics, 63, 1331-1341, doi:10.1016/S1364-6826(00)00251-0.

Dong, Z. and S. Jin, 2018: 3-D water vapor tomography in Wuhan from GPS, BDS and GLONASS observations. Remote Sensing, 10, 62, doi:10.3390/rs10010062. 
Ducrocq, V., D. Ricard, J. P. Lafore, and F. Orain, 2002: Storm-scale numerical rainfall prediction for five precipitating events over France: On the importance of the initial humidity field. Weather and Forecasting, 17, 1236-1256.

E-GVAP, 2014: COST-Format File Specification for Ground-based GNSS Delay and Water Vapour Data. https://www.romsaf .org/egvap_cost.pdf.

Elgered, G., 2001: An overview of COST Action 716: exploitation of ground-based GPS for climate and numerical weather prediction applications. Physics and Chemistry of the Earth, Part A: Solid Earth and Geodesy, 26(6-8), 399-404.

Essen, L. and K. D. Froome, 1951: The Refractive Indices and Dielectric Constants of Air and its Principal Constituents at 24,000 Mc/s. Proceedings of the Physical Society. Section B, 64 (10), 862-875, doi:10.1088/0370-1301/64/10/303, URL https:// doi .org/10.1088\%2F0370-1301\%2F64\%2F 10\%2F303.

Farooq, S. Z., D. Yang, T. Jin, and N. Echoda, 2018: Survey of Cycle Slip Detection and Correction Techniques for Single Frequency Receivers. 957-961, doi:10.1109/ICCT. 2018.8599879 .

Fujita, M., F. Kimura, K. Yoneyama, and M. Yoshizaki, 2008: Verification of precipitable water vapor estimated from shipborne GPS measurements. Geophysical Research Letters, 35 (13), doi:10.1029/2008GL033764.

Fujiwara, M., et al., 2017: Introduction to the SPARC Reanalysis Intercomparison Project (S-RIP) and overview of the reanalysis systems. Atmospheric Chemistry and Physics, 17, 1417-1452, doi:10.5194/acp-17-1417-2017.

Gao, Y., Y. Zhang, and K. Chen, 2006: Development of a Real-Time Single-Frequency Precise Point Positioning System and Test Results.

Gao, Z., M. Ge, and W. Shen, 2017: lonospheric and receiver DCB-constrained multiGNSS single-frequency PPP integrated with MEMS inertial measurements. Journal of Geodesy, 91, 1351—-1366, doi:https://doi.org/10.1007/s00190-017-1029-7.

Ge, M., G. Gendt, M. Rothacher, S. Changhong, and J. Liu, 2008: Resolution of GPS Carrier-Phase Ambiguities in Precise Point Positioning (PPP) with Daily Observations. Journal of Geodesy, 82, 389-399, doi:10.1007/s00190-007-0187-4.

Glaner, M. F., 2017: Eigenschaften und Einsatzmöglichkeiten von GPS und Galileo Signal-Linearkombinationen. M.S. thesis, Department für Geodäsie und Geoinfor- 
mation / Höhere Geodäsie, URL http://repositum.tuwien.ac.at/urn:nbn:at: at-ubtuw : 1-96172.

Glaner, M. F., R. Weber, and S. Strasser, 2020: PPP-AR with GPS and Galileo: Assessing diverse approaches and satellite products to reduce convergence time. EGU General Assembly Conference Abstracts, 7792, EGU General Assembly Conference Abstracts.

Graham, E., E. N. Koffi, and C. Matzler, 2012: An observational study of air and water vapour convergence over the western Alps during summer and the development of isolated thunderstorms. Meteorologische Zeitschrift, 21, 1-13.

Guerova, G., et al., 2016: Review of the state-of-the-art and future prospects of the ground-based GNSS meteorology in Europe. Atmospheric Measurement Techniques Discussions, 9, doi:10.5194/amt-9-5385-2016.

Gurtner, W. and L. Estey, 2007: Rinex: The receiver independent exchange format version 2.11. Astronomical Institute, University of Bern, Bern.

Hadas, T., T. Hobiger, and P. Hordyniec, 2020: Considering different recent advancements in GNSS on real-time zenith troposphere estimates. GPS Solutions, 24, doi: 10.1007/s10291-020-01014-w.

Haltiner, G. J. and F. L. Martin, 1957: Dynamical and Physical Meteorology. McGrawHill Book Company.

Hanna, N., E. Trzcina, G. Möller, W. Rohm, and R. Weber, 2019: Assimilation of GNSS tomography products into the Weather Research and Forecasting model using radio occultation data assimilation operator. Atmospheric Measurement Techniques, 12 (9), 4829-4848, doi:10.5194/amt-12-4829-2019, URL https://www. atmos-meas-tech.net/12/4829/2019/.

Herrera Olmo, A., H. Suhandri, E. Realini, M. Reguzzoni, and M. C. d. Lacy, 2015: goGPS: open-source MATLAB software. GPS Solutions, 20, 1-9, doi:10.1007/ s10291-015-0469-x.

Herring, T. A., 1992: Modelling atmospheric delays in the analysis of space geodetic data. Symposium on Refraction of Transatmospheric Signalsin Geodesy, 36, 157-164. 
Hersbach, H., et al., 2020: The ERA5 global reanalysis. Quarterly Journal of the Royal Meteorological Society, 146 (730), 1999-2049, doi:10.1002/qj.3803, https: //rmets.onlinelibrary.wiley.com/doi/pdf/10.1002/qj.3803.

Hinterberger, F., 2016: Influence of GPS satellite orbits and clock corrections on the estimation of single difference uncalibrated phase delays. Ph.D. thesis, Technische Universität Wien.

Hlawatsch, F., 2019: Lecture notes in Parameter Estimation Methods. Institute of Telecommunications, Technical University Vienna.

Hofmann-Wellenhof, B., H. Lichtenegger, and J. Collins, 2012: Global Positioning System: Theory and Practice. Springer Vienna, URL https://books.google.at/ books?id=F7jrCAAAQBAJ.

Hofmeister, A., 2016: Determination of path delays in the atmosphere for geodetic VLBI by means of ray-tracing. Ph.D. thesis, Department f. Geodäsie u. Geoinformation / Höhere Geodäsie, URL http://resolver.obvsg.at/urn:nbn:at:at-ubtuw: $1-3444$.

Huang, G., K. Eckenhoff, and J. Leonard, 2018: Optimal-State-Constraint EKF for Visual-Inertial Navigation. In: Bicchi A., Burgard W. (eds) Robotics Research. Springer Proceedings in Advanced Robotics, 2, doi:https://doi.org/10.1007/ 978-3-319-51532-8_8.

Huang, X. Y., et al., 2003: TOUGH: Targeting optimal use of GPS humidity measurements in meteorology. International Workshop on GPS Meteorology.

Jackson, J. D., 1999: Classical electrodynamics. 3d ed., Wiley.

Jiang, C., T. Xu, S. Wang, W. Nie, and S. Zhangzhen, 2020: Evaluation of Zenith Tropospheric Delay Derived from ERA5 Data over China Using GNSS Observations. Remote Sensing, 12, 663, doi:10.3390/rs12040663.

Jin, S. and O. Luo, 2009: Variability and climatology of PWV from global 13-year GPS observations. IEEE Trans. Geosci. Remote Sens. 47 (7).

Kalman, R. E., 1960: A new approach to linear filtering and prediction problems. Transactions of the ASME-Journal of Basic Engineering, 82 (Series D), 35-45.

Kaplan, E. and C. Hegarty, 2006: Understanding GPS - Principles and Applications. Artech House. 
Karabatic, A., 2011: Precis Point Positioning (PPP) - an alternative technique for ground based GNSS troposphere monitoring. Ph.D. thesis, Geodäsie und Geophysik.

Katsigianni, G., F. Perosanz, S. Loyer, and M. Gupta, 2019: Galileo millimeter-level kinematic precise point positioning with ambiguity resolution. Earth, Planets and Space, 71, doi:10.1186/s40623-019-1055-1.

Kačmařík, M., et al., 2017: Inter-technique validation of tropospheric slant total delays. Atmospheric Measurement Techniques, 10 (6), 2183-2208, doi:10.5194/ amt-10-2183-2017, URL https://www . atmos-meas-tech.net/10/2183/2017/.

Kealy, J., J. Foster, and S. Businger, 2012: GPS meteorology: An investigation of oceanbased precipitable water estimates. Journal of Geophysical Research: Atmospheres, 117 (D17), doi:10.1029/2011JD017422.

Kouba, J. and P. Héroux, 2001: Precise Point Positioning Using IGS Orbit and Clock Products. GPS Solutions, 5, 12-28, doi:10.1007/PL00012883.

Krawinkel, T., N. Lindenthal, and S. Schön, 2014: Apparent coordinate changes of gps reference stations: Influence of evaluation strategies and antenna changes. ZFV - Zeitschrift fur Geodasie, Geoinformation und Landmanagement, 139, 252-263, doi: $10.12902 / \mathrm{zfv}-0027-2014$.

Krietemeyer, A., M.-c. Ten Veldhuis, H. Marel, E. Realini, and N. van de Giesen, 2018: Potential of Cost-Efficient Single Frequency GNSS Receivers for Water Vapor Monitoring. Remote Sensing, 10, 1493, doi:10.3390/rs10091493.

Landskron, D. and J. Böhm, 2017: VMF3/GPT3: refined discrete and empirical troposphere mapping functions. Journal of Geodesy, 92, 349-360.

Laurichesse, D., F. Mercier, J.-P. Berthias, P. Broca, and L. Cerri, 2009: Integer Ambiguity Resolution on Undifferenced GPS Phase Measurements and Its Application to PPP and Satellite Precise Orbit Determination. Navigation, 56 (2), 135-149, doi: 10.1002/j.2161-4296.2009.tb01750.x.

Laurichesse, D. and A. Privat, 2015: An Open-source PPP Client Implementation for the CNES PPP-WIZARD Demonstrator.

Li, Z., S. P. Ballard, and D. Simonin, 2018: Comparison of 3D-Var and 4D-Var data assimilation in an NWP-based system for precipitation nowcasting at the Met 
Office. Quarterly Journal of the Royal Meteorological Society, 144 (711), 404413, doi:10.1002/qj.3211, https://rmets.onlinelibrary.wiley.com/doi/pdf/ $10.1002 / q j .3211$.

Lu, C., X. Chen, G. Liu, G. Dick, J. Wickert, X. Jiang, Z. Kai, and H. Schuh, 2017: Real-Time Tropospheric Delays Retrieved from Multi-GNSS Observations and IGS Real-Time Product Streams. Remote Sensing, 9, 1317, doi:10.3390/rs9121317.

Magnet, N., 2019: Giomo: A robust modelling approach of ionospheric delays for GNSS real-time positioning applications. Ph.D. thesis, Department für Geodäsie und Geoinformation / Höhere Geodäsie, URL https://repositum.tuwien.ac.at/urn:nbn: at : at-ubtuw: 1-124443.

Marini, J., 1972: Correction of satellite tracking data for an arbitrary tropospheric profile. Radio Science, 7, 223-231.

Melbourne, W., 1985: The case for ranging in gps based geodetic systems. Proceedings of1st International Symposium on Precise Positioning with the Global Positioning System, 373-386.

Mendez Astudillo, J., L. Lau, Y.-T. Tang, and T. Moore, 2018: Analysing the Zenith Tropospheric Delay Estimates in On-line Precise Point Positioning (PPP) Services and PPP Software Packages. Sensors, 18, 580, doi:10.3390/s18020580.

MeteoSwiss, 2020: COSMO forecasting system. https://www. meteoswiss.admin.ch/home/measurement-and-forecasting-systems/ warning-and-forecasting-systems/cosmo-forecasting-system.html.

Mile, M., P. Benáček, and S. Rózsa, 2019: The use of GNSS zenith total delays in operational AROME/Hungary 3D-Var over a central European domain. Atmospheric Measurement Techniques, 12 (3), 1569-1579, doi:10.5194/amt-12-1569-2019, URL https://www . atmos-meas-tech.net/12/1569/2019/.

Möller, G., 2017: Reconstruction of 3D wet refractivity fields in the lower atmosphere along bended GNSS signal paths. Ph.D. thesis, Department für Geodäsie und Geoinformation / Höhere Geodäsie, URL http://repositum.tuwien.ac.at/obvutwhs/ content/titleinfo/2268559.

Najman, P. and T. Kos, 2014: Performance Analysis of Empirical lonosphere Models by Comparison with CODE Vertical TEC Maps, Mitigation of lonospheric Threats to GNSS. 
National Imagery and Mapping Agency, 2000: Department of defense world geodetic system 1984: its definition and relationships with local geodetic systems. Tech. Rep. TR8350.2, National Imagery and Mapping Agency, St. Louis, MO, USA. URL http: //earth-info.nga.mil/GandG/publications/tr8350.2/tr8350_2.html.

Niell, A., 1996: Global mapping functions for the atmosphere delay at radio wavelengths. J. Geophys. Res., 101, 3227-3246.

Niell, A., 2000: Improved atmospheric mapping functions for VLBI and GPS. Earth Planets Space, 52, 699-702.

Nilsson, T., J. Böhm, D. Wijaya, A. Tresch, V. Nafisi, and H. Schuh, 2013: Path Delays in the Neutral Atmosphere. In: Böhm J., Schuh H. (eds) Atmospheric Effects in Space Geodesy. Springer Atmospheric Sciences. Springer, Berlin, Heidelberg, 73-136.

Realini, E. and M. Reguzzoni, 2013: goGPS: Open Source Software for Enhancing the Accuracy of Low-cost Receivers by Single-frequency Relative Kinematic Positioning. Measurement Science and Technology, 24, 115010, doi:10.1088/0957-0233/24/11/ 115010.

Rüeger, J., 2002: Refractive Index Formulae for Radio Waves. Proc. FIG XXII International Congress, Washington, D. C.

Rocken, C., J. Johnson, T. Van Hove, and T. Iwabuchi, 2005: Atmospheric water vapor and geoid measurements in the open ocean with GPS. Geophysical Research Letters, 32 (12), doi:10.1029/2005GL022573.

Rohm, W., J. Guzikowski, K. Wilgan, and M. Kryza, 2019: 4DVAR assimilation of GNSS zenith path delays and precipitable water into a numerical weather prediction model WRF. Atmospheric Measurement Techniques, 12 (1), 345-361, doi:10.5194/ amt-12-345-2019, URL https://www .atmos-meas-tech.net/12/345/2019/.

Saastamoinen, J., 1972: Contributions to the theory of atmospheric refraction. Bull. Geodesique, 105, 13-34.

Santos, M., D. McAdam, and J. Böhm, 2012: Status and Validation of the UNBVMF1. EGU General Assembly Conference Abstracts, 13759, EGU General Assembly Conference Abstracts.

Sanz Subirana, J., J. M. Juan Zornoza, and M. Hernández-Pajares, 2013: GNSS Data Processing, Volume I: Fundamentals and Algorithms. ESA Communications, ESTEC, Noordwijk, Netherlands. 
Schönemann, E., 2014: Analysis of GNSS raw observationsin PPP solutions. Ph.D. thesis, Technische Universität Darmstadt.

Seco, A., et al., 2012: Rain pattern analysis and forecast model based on GPS estimated atmospheric water vapor content. Atmospheric Environment, 49, 85-93.

Simeonov, T., D. Sidorov, F. N. Teferle, G. Milev, and G. Guerova, 2016: Evaluation of IWV from the numerical weather prediction WRF model with PPP GNSS processing for Bulgaria. Atmospheric Measurement Techniques Discussions, 2016, 115, doi:10.5194/amt-2016-152, URL https://amt.copernicus.org/preprints/ amt-2016-152/.

Skamarock, W. C., J. B. Klemp, J. Dudhia, D. O. Gill, Z. Liu, J. Berner, and X. Huang, 2019: A Description of the Advanced Research WRF Model Version 4. Tech. rep., NCAR. doi:10.5065/1dfh-6p97.

Skone, S., Y. Gao, O. Al-Fanek, W. Tao, Y. Zhang, P. Héroux, and P. Collins, 2006: Atmospheric moisture estimation using GPS on a moving platform. 3, 1891-1901.

Smith, E. K. and S. Weintraub, 1953: The Constants in the Equation for Atmospheric Refractive Index at Radio Frequencies. Journal of Research of the Notional Bureau of Standards, 50, 39-41.

Ssenyunzi, R. C., B. Oruru, F. M. D'ujanga, E. Realini, S. Barindelli, G. Tagliaferro, A. von Engeln, and N. van de Giesen, 2020: Performance of ERA5 data in retrieving Precipitable Water Vapour over East African tropical region. Advances in Space Research, 65 (8), 1877 - 1893, doi:https://doi.org/10.1016/j.asr.2020.02.003.

Stepniak, K. and O. Bock, 2018: Comparison of tropospheric estimates from DD and PPP processing approaches for climate monitoring. EGU General Assembly Conference Abstracts, 14722, EGU General Assembly Conference Abstracts.

Suya, G., 2019: lonospheric-Constrained PPP using Triple-GNSS Constellations.

Takasu, T. and A. Yasuda, 2009: Development of the low-cost RTK-GPS receiver with an open source program package RTKLIB. International Symposium on GPS/GNSS.

Thiruvengadam, P., J. Indu, and S. Ghosh, 2020: Significance of 4DVAR Radar Data Assimilation in Weather Research and Forecast Model-Based Nowcasting System. Journal of Geophysical Research: Atmospheres, 125 (11), e2019JD031369, doi:10.1029/2019JD031369, https://agupubs.onlinelibrary.wiley.com/doi/ $\operatorname{pdf} / 10.1029 / 2019 J D 031369$. 
Tétreault, P., J. Kouba, P. Héroux, and P. Legree, 2005: CSRS-PPP: An internet service for GPS user access to the Canadian Spatial Reference frame. Geomatica, 59, 17-28.

van Baelen, J., M. Reverdy, F. Tridon, L. Labbouz, G. Dick, M. Bender, and M. Hagen, 2011: On the relationship between water vapour and evolution and the life cycle of precipitation systems. Q. J. R. Meteorol. Soc, 137, 204-223.

Vedel, H. and X.-Y. Huang, 2004: Impact of Ground Based GPS Data on Numerical Weather Prediction. Journal of the Meteorological Society of Japan. Ser. II, 82 (1B), 459-472, doi:10.2151/jmsj.2004.459.

Virtanen, P., et al., 2020: SciPy 1.0: Fundamental Algorithms for Scientific Computing in Python. Nature Methods, 17, 261-272, doi:10.1038/s41592-019-0686-2.

Wallace, J. M. and P. V. Hobbs, 2006: Atmospheric Science: An Introduction Survey. Elsevier, 255-280 pp.

Webb, S. R., N. T. Penna, P. J. Clarke, S. Webster, I. Martin, and G. V. Bennitt, 2016: Kinematic GNSS Estimation of Zenith Wet Delay over a Range of Altitudes. Journal of Atmospheric and Oceanic Technology, 33 (1), 3-15, doi: 10.1175/JTECH-D-14-00111.1.

Weber, R., D. Landskron, N. Hanna, M. Aichinger-Rosenberger, and D. Horozovic, 2019: Atmosphere Monitoring by means of GNSS - Research Activities at TU Wien. Österreichische Zeitschrift für Vermessung und Geoinformation (VGI), 107 (2), 7882.

Wickert, J., et al., 2009: GPS radio occultation: results from CHAMP, GRACE and FORMOSAT-3/COSMIC. Terrestrial Atmospheric and Oceanic Sciences, 20 (1), 3550, doi:https://doi.org/10.3319/TAO.2007.12.26.01(F3C).

Wilgan, K. and A. Geiger, 2018: High-resolution models of tropospheric delays and refractivity based on GNSS and numerical weather prediction data for alpine regions in Switzerland. Journal of Geodesy, doi:10.1007/s00190-018-1203-6.

Wilks, D. S., 2011: Statistical methods in the Atmospheric Sciences. Elsevier, 255-280 pp.

Wolfe, D. E. and S. E. Gutman, 2000: Developing an Operational, Surface-Based, GPS, Water Vapor Observing System for NOAA: Network Design and Results. Journal of Atmospheric and Oceanic Technology, 17, 426-440. 
Wu, J. T., S. C. Wu, G. A. Hajj, W. I. Bertiger, and S. M. Lichten, 1992: Effects of antenna orientation on GPS carrier phase. Astrodynamics 1991, 1647-1660.

Wulfmeyer, V., et al., 2008: The convective and orographically induced precipitation study: A research and development project of the World Weather Research Program for improving quantitative precipitation forecasting in low-mountain regions. Bull. Amer. Meteorol. Soc., 89(10), 1477-1486.

Xu, G., 2007: Gps: Theory, algorithms and applications. GPS: Theory, Algorithms and Applications, 1-340, doi:10.1007/978-3-540-72715-6.

Xu, Y., 2016: GNSS Precise Point Positioning with Application of the Equivalence Principle. Ph.D. thesis, Technischen Universität Berlin.

Yan, X., V. Ducrocq, G. Jaubert, P. Brousseau, P. Poli, C. Champollion, C. Flamant, and K. Boniface, 2009: The benefit of GPS zenith delay assimilation to high-resolution quantitative precipitation forecast. A case-study from COPS IOP 9. Q. J. R. Meteorol. Soc., 135, 1788-1800.

Yu, X. and J. Gao, 2017: Kinematic Precise Point Positioning Using Multi-Constellation Global Navigation Satellite System (GNSS) Observations. ISPRS International Journal of Geo-Information, 6, 6, doi:10.3390/ijgi6010006.

Zhao, C., B. Zhang, W. Li, Y. Yuan, and M. Li, 2019: Simultaneous Retrieval of PWV and VTEC by Low-Cost Multi-GNSS Single-Frequency Receivers. Earth and Space Science, 6 (9), 1694-1709, doi:10.1029/2019EA000650, https://agupubs . onlinelibrary.wiley.com/doi/pdf/10.1029/2019EA000650.

Zhao, Q., Y. Yao, X. Cao, F. Zhou, and P. Xia, 2018: An Optimal Tropospheric Tomography Method Based on the Multi-GNSS Observations. Remote Sensing, 10 (2), 2072-4292, doi:10.3390/rs10020234, URL https://www.mdpi.com/2072-4292/ $10 / 2 / 234$.

Zhou, F., D. Dong, W. li, X. Jiang, J. Wickert, and H. Schuh, 2018: GAMP: An open-source software of multi-GNSS precise point positioning using undifferenced and uncombined observations. GPS Solutions, 22, doi:10.1007/s10291-018-0699-9.

Zhu, K., X. Guo, C. Jiang, Y. Xue, Y. Li, L. Han, and Y. Chen, 2020: Mimu/odometer fusion with state constraints for vehicle positioning during beidou signal outage: Testing and results. Sensors (Basel, Switzerland), 20, doi:https://doi.org/10.3390/ s20082302. 
Zumberge, J., M. Heflin, D. Jefferson, M. Watkins, and F. Webb, 1997: Precise Point Positioning for the Efficient And Robust Analysis of GPS Data from Large Networks. Journal of Geophysical Research, 102, doi:10.1029/96JB03860.

Zus, F., J. Douša, M. Kačmařík, P. Václavovic, K. Balidakis, G. Dick, and J. Wickert, 2019a: Improving GNSS Zenith Wet Delay Interpolation by Utilizing Tropospheric Gradients: Experiments with a Dense Station Network in Central Europe in the Warm Season. Remote Sensing, 11 (6), doi:10.3390/rs11060674.

Zus, F., J. Douša, M. Kačmařík, P. Václavovic, G. Dick, and J. Wickert, 2019b: Estimating the Impact of Global Navigation Satellite System Horizontal Delay Gradients in Variational Data Assimilation. Remote Sensing, 11 (1), doi:10.3390/rs11010041. 


\section{Curriculum Vitae}

Matthias Aichinger-Rosenberger

Ried 14/7, 6306 Söll

Born on 30 June 1990 in St.Pölten, Austria

\section{Education and Professional training:}

01/2020 - Technical certifier for GNSS-based applications at NavCert GmbH, Munich, Germany.

02/2018- Project assistant and Ph.D. student in the group of Prof. Dr. Robert

12/2019 Weber at the Research division Higher Geodesy, Department of Geodesy and Geoinformation, Technical University Vienna.

2017-2018 Master thesis under the guidance of Prof. Dr. Mathias Rotach, Institute of Meteorology and Geophysics, University of Innsbruck: Usability of highresolution GNSS-ZTD data in the AROME model.

2015-2018 Master studies at the University of Innsbruck. Master of Science (M.Sc) in Atmospheric sciences.

2014-2015 Master thesis under the guidance of Prof. Dr. Johannes Böhm and Dr. Michael Schindelegger, Institute of Geodesy and Geoinformation, Technical University Vienna: "Atmospheric contribution to decadal-scale polar motion variations from twentieth century reanalysis".

2013-2015 Master studies at Technical University Vienna. Master of Science (Dipl.Ing.) in Geodesy and Geoinformation.

2000-2008 Bunderealgymnasium, Krems. Matura.

\section{Training courses:}

"Parallel programming using MPI", Vienna Scientific Cluster (VSC), 2019;

"Parallel I/O", Vienna Scientific Cluster (VSC), 2019. 